\title{
DEVELOPMENT OF A SIMULTANEOUS DESIGN FOR SUPPLY CHAIN PROCESS FOR THE OPTIMIZATION OF THE PRODUCT DESIGN AND SUPPLY CHAIN CONFIGURATION PROBLEM
}

\author{
by \\ Nuri Mehmet Gökhan \\ B.S., Istanbul Technical University, 2001 \\ M.S., Sabanci University, 2003 \\ Submitted to the Graduate Faculty of \\ the School of Engineering in partial fulfillment \\ of the requirements for the degree of \\ Doctor of Philosophy
}

University of Pittsburgh 


\section{UNIVERSITY OF PITTSBURGH \\ SCHOOL OF ENGINEERING}

This dissertation was presented

by

Nuri Mehmet Gökhan

It was defended on

September 14, 2007

and approved by

Kim LaScola Needy, PhD, Associate Professor, Department of Industrial Engineering

Bryan A. Norman, PhD, Associate Professor, Department of Industrial Engineering

Brady Hunsaker, PhD, Assistant Professor, Department of Industrial Engineering

Michael R. Lovell, PhD, Professor, Department of Industrial Engineering

Robert J. Ries, PhD, Research Assistant Professor, Department of Civil and Environmental

Engineering

Dissertation Director: Kim LaScola Needy, Ph.D., Associate Professor, Department of

Industrial Engineering 
Copyright (C) by Nuri Mehmet Gökhan

2007 


\section{DEVELOPMENT OF A SIMULTANEOUS DESIGN FOR SUPPLY CHAIN PROCESS FOR THE OPTIMIZATION OF THE PRODUCT DESIGN AND SUPPLY CHAIN CONFIGURATION PROBLEM}

Nuri Mehmet Gökhan, PhD

University of Pittsburgh, 2007

This research investigates the development of a process for Design for Supply Chain (DFSC) - a process that aims to reduce the product life cycle costs, improve product quality, improve efficiency, and improve profitability for all partners in the supply chain (SC). It focuses on understanding the impacts and benefits of incorporating the $\mathrm{SC}$ configuration problem into the product design phase. As the product design establishes different requirements on the manufacturability, cost, and similar parameters, the SC is also closely linked to product design decisions and impacted by them. This research uniquely combines the impacts of the product design and price decisions on the product demand and the impacts of the SC decisions on cost, lead time, and demand satisfaction.

The developed mathematical models are aimed at economically managing the SC for product design and support not only product design, but also redesign associated with process improvements and design changes in general. This research suggests development of a proactive approach to product design allowing impacts to the SC to be predicted in advance and resolved more quickly and economically. It presents two product and SC design approaches. The sequential approach examines the design of a product followed by the SC design where the simultaneous approach considers both the product and SC designs concurrently. By utilizing Mixed Integer Programming and a Genetic Algorithm, this research studies various research questions which examine modeling preferences and essential performance metrics, impacts of 
using a sequential versus simultaneous design approach on these performance metrics, the robustness of the resulting SC design, and relative importance of the product and SC design on the profits. To answer these questions, different models are developed, tested with illustrative data, and the results are analyzed.

The test results and industry experts' validations conclude that the developed DFSC models add significant value to the product design procedure resulting in a useful decision support tool. The results indicate that the simultaneous DFSC approach captures the complex interactions between the product and supply chain decisions, improving the overall profit of a product across its life cycle.

Keywords: Design for Supply Chain, Product Design, Supply Chain, Simultaneous Optimization, Mixed Integer Programming, Heuristics, Genetic Algorithm. 


\section{TABLE OF CONTENTS}

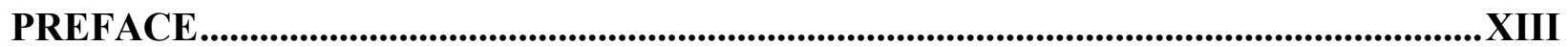

NOTATION

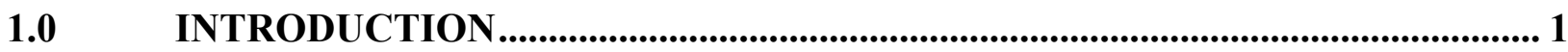

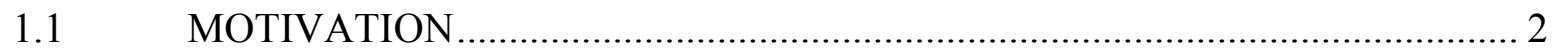

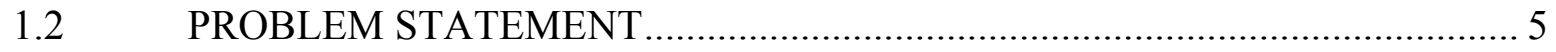

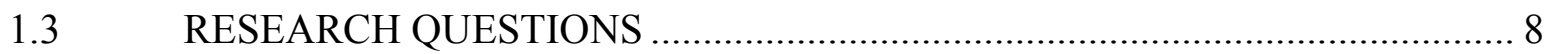

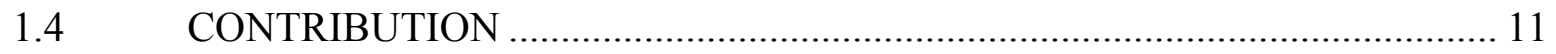

$1.5 \quad$ OVERVIEW OF THE DISSERTATION ……........................................... 12

$2.0 \quad$ LITERATURE REVIEW...................................................................................... 14

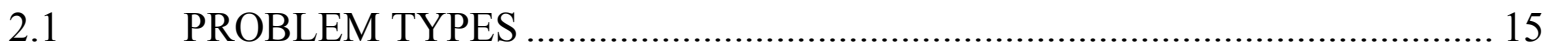

2.1.1 Product Design Models ............................................................................ 15

2.1.2 Supply Chain Models........................................................................ 19

2.1.3 Product Design and Supply Chain Combined Models …………………..... 22

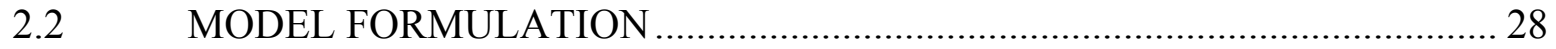

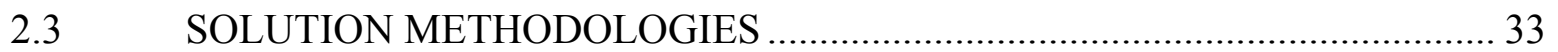

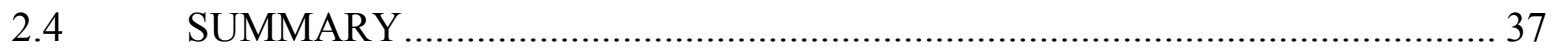

3.0 PROBLEM FORMULATION.................................................................................... 40

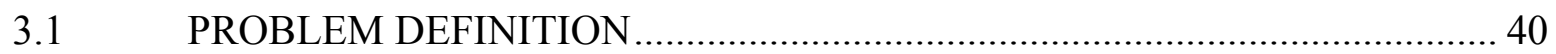




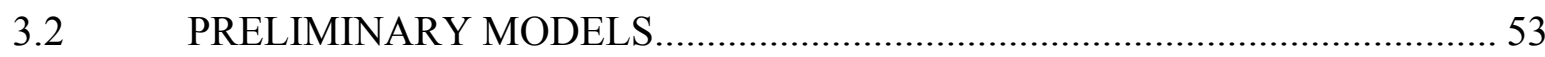

3.3 COMPLETE MODEL FORMULATION …….......................................... 58

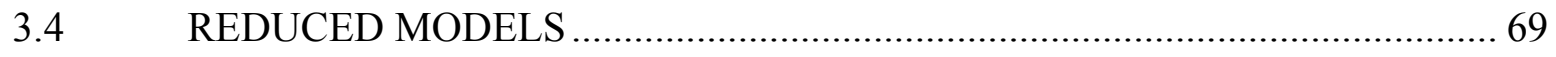

3.5 MODEL ASYMPTOTIC SIZE ANALYSIS …………………………….... 71

4.0 SOLUTION METHODOLOGY .................................................................... 77

4.1 DETERMINISTIC OPTIMIZATION METHODOLOGIES ............................... 77

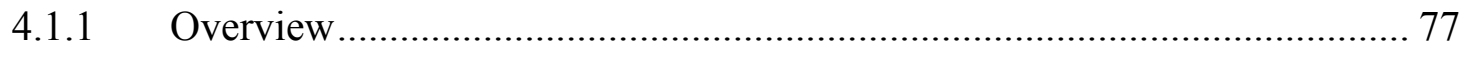

4.1.2 Performance on the Preliminary Models ……………................................ 79

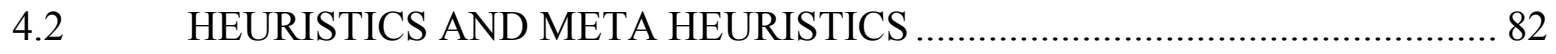

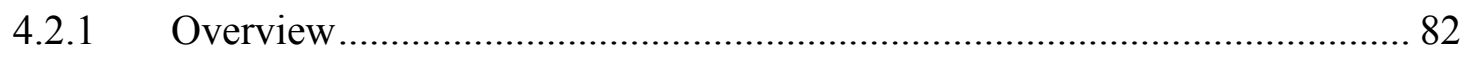

4.2.2 Performance on the Preliminary Models ………........................................ 86

4.3 SELECTED SOLUTION METHODOLOGIES AND IMPLEMENTATION... 96

4.3.1 Mixed Integer Programming ..................................................................... 97

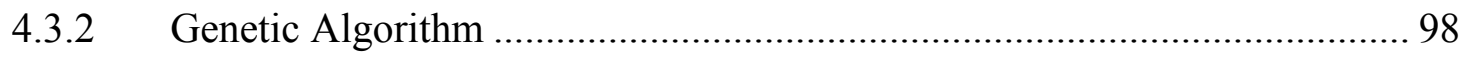

4.4 SOLUTION TECHNIQUES COMPLEXITY ANALYSIS............................... 107

5.0 COMPUTATIONAL RESULTS ....................................................................... 110

5.1 PERFORMANCE TESTS ON COMPLETE AND REDUCED MODELS ..... 110

5.1.1 Mixed Integer Programming ...................................................................... 111

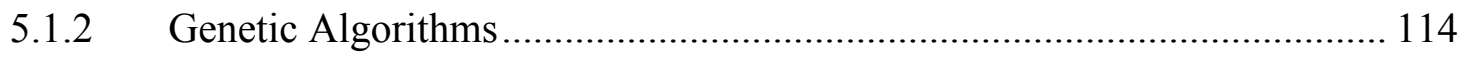

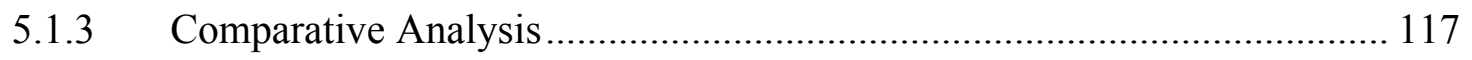

5.2 PROBLEM AND MODEL VALIDATION ………………............................. 128

5.3 ANSWERS TO RESEARCH QUESTIONS ................................................ 132

5.3.1 Performance Metrics and Modeling Preferences ........................................ 133 
5.3.2 Simultaneous versus Sequential Approach...................................... 142

5.3.3 Combining Product and Supply Chain Design ..................................... 155

6.0 CONCLUSIONS AND FUTURE RESEARCH ................................................. 159

SUMMARY AND CONCLUSIONS .................................................. 159

6.2 EXTENSIONS FOR DFSC MODELS AND SOLUTION METHODS ......... 162

APPENDIX A. MODEL LINEARIZATION SCHEMA ................................................ 166

APPENDIX B. REDUCED DFSC MODELS ................................................................... 173

APPENDIX C. PRELIMINARY GA PARAMETER TEST RESULTS ............................ 179

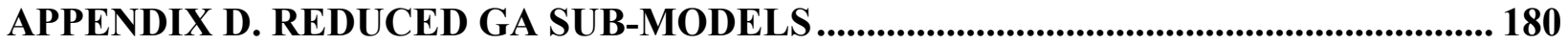

BIBLIOGRAPHY ............................................................................................................. 183 


\section{LIST OF TABLES}

1. Performance measures for supply chain management (adapted from [56]) .......................... 32

2. Summary of number of variables and constraints in different models .............................. 75

3. Numerical illustration of model asymptotic size of different models ................................. 76

4. Illustrative test problem data for the preliminary models........................................... 80

5. MIP cost minimization model preliminary run results .............................................. 81

6. MIP lead time minimization model preliminary run results.......................................... 81

7. Genetic Algorithm cost minimization model preliminary run results .............................. 91

8. Genetic Algorithm lead time minimization model preliminary run results.......................... 91

9. Tabu Search cost minimization model preliminary run results ....................................... 94

10. Tabu Search lead time minimization model preliminary run results............................... 95

11. Summary of number of variables and constraints in GA sub-models ............................. 102

12. Numerical illustration of model asymptotic size of GA sub-models .............................. 103

13. Complete and reduced DFSC problem instances for performance testing ....................... 111

14. MIP results for the complete DFSC model............................................................ 112

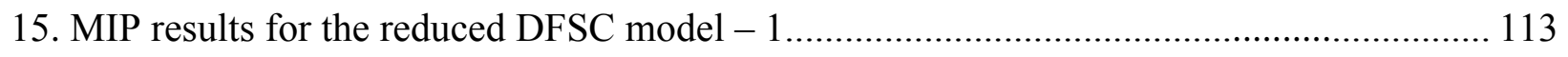

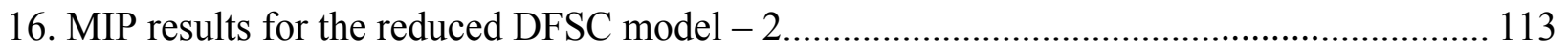

17. GA results for the complete DFSC model ........................................................... 115 
18. GA results for the reduced DFSC model - 1 ........................................................................ 116

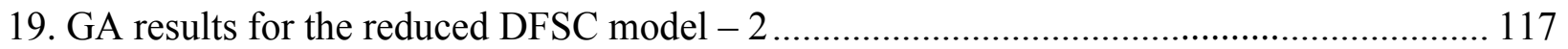

20. MIP results for the complete and reduced DFSC models................................................... 133

21. GA results for the complete and reduced DFSC models ..................................................... 134

22. Customer satisfaction values of component design alternatives - cordless phone .............. 137

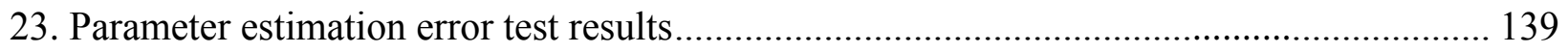

24. Parameter estimation error test results by using the base case solution ............................... 141

25. Customer satisfaction values of component design alternatives - desktop computer ......... 144

26. Simultaneous and sequential approach results for the cordless phone example .................. 147

27. Simultaneous and sequential approach results for the desktop computer example............... 148

28. Simultaneous and sequential approach results with alternative sequential procedure ..........151

29. Optimal solutions of simultaneous and sequential approaches - desktop computer............ 152

30. Revenue, costs, and lead time results of the cordless phone example.................................. 153

31. Revenue, costs, and lead time results of the desktop computer example ............................. 153

32. Product design and supply chain decisions for the cordless phone example ........................ 156

33. Product design and supply chain decisions for the desktop computer example.................... 157 


\section{LIST OF FIGURES}

1. Actions affecting life cycle cost (adapted from [34]) .................................................... 3

2. Sequential and simultaneous product and supply chain design processes ........................... 7

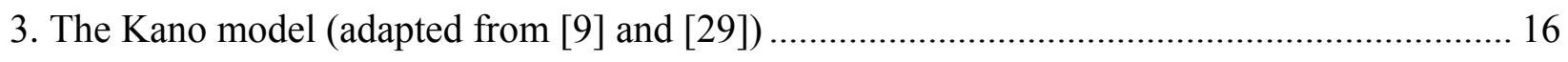

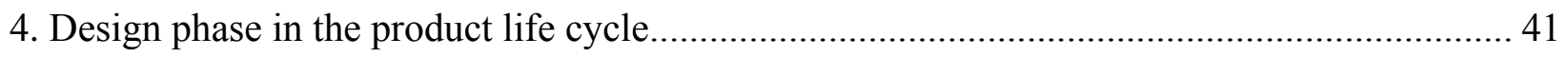

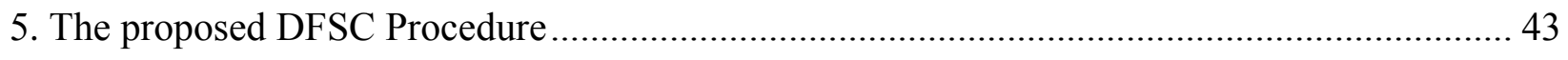

6. Chromosome structure of the Genetic Algorithm for the preliminary models ...................... 87

7. Chromosome structure of the Genetic Algorithm for complete and reduced models............. 99

8. Progress of the MIP and the GA for the complete DFSC model (instance 1) ..................... 118

9. Progress of the MIP and the GA for the reduced DFSC model - 1 (instance 1) .................. 119

10. Progress of the MIP and the GA for the reduced DFSC model - 2 (instance 1) ................ 119

11. Progress of the MIP and the GA for the complete DFSC model (instance 2) .................... 120

12. Progress of the MIP and the GA for the reduced DFSC model - 1 (instance 2) ................. 121

13. Progress of the MIP and the GA for the reduced DFSC model - 2 (instance 2) ................ 121

14. Progress of the MIP and the GA for the complete DFSC model (instance 3) ................... 122

15. Progress of the MIP and the GA for the reduced DFSC model - 1 (instance 3) ................ 123

16. Progress of the MIP and the GA for the reduced DFSC model - 2 (instance 3) ................ 123

17. Progress of the MIP and the GA for the complete DFSC model (instance 4) ................... 124 
18. Progress of the MIP and the GA for the reduced DFSC model - 1 (instance 4)

19. Progress of the MIP and the GA for the reduced DFSC model - 2 (instance 4)

20. Progress of the MIP and the GA for the complete DFSC model (instance 5)

21. Progress of the MIP and the GA for the reduced DFSC model - 1 (instance 5)

22. Progress of the MIP and the GA for the reduced DFSC model - 2 (instance 5)

23. The sequential approach procedure based on separate time periods. 146

24. The sequential approach procedure based on the optimization over all time periods 150 


\section{PREFACE}

Though only my name appears on the cover of this dissertation, a great many people have contributed to its production. I owe my gratitude to all those people who have made this dissertation possible and because of whom my graduate experience has been one that I will cherish forever.

I would like to express my deepest gratitude to my advisor and mentor, Dr. Kim L. Needy for her endless guidance, support, patience, and direction throughout my dissertation research, as well as other studies. I have been amazingly fortunate to have an advisor who gave me the freedom to explore on my own and at the same time the guidance to recover when my steps faltered. Without her support and inspiration, I would not be able to accomplish what I have done. I would also like to thank her for her enthusiasm, sincere advice, and invaluable suggestions that guided me through countless difficulties in all aspects of my life.

I am also grateful to my committee members, Drs. Bryan Norman, Brady Hunsaker, Robert Ries, and Michael Lovell for their support, help, and guidance throughout my research. I would like to thank them for advising me in making this dissertation more accurate and more solid.

I would like to thank University of Pittsburgh's NSF Center for e-Design and Drs. Bart O. Nnaji and Michael Lovell for providing me the opportunity to work under the Center's 
support throughout my research. I would also like to thank industry experts who provided invaluable input and feedback for my dissertation.

I gladly express my gratitude to the wonderful Industrial Engineering staff, Richard Brown, Minerva Pilachowski, Nora Siewiorek, Lisa Bopp, and Chalice Zavada for providing support and help during my long years at Pitt. I want to express my sincere thanks to Richard and Nora not only for their enthusiasm but also for all the coffee that kept me awake all the time.

I am very grateful to all my friends in the Department who always supported me and created a joyful work environment. I would like to thank Özlem Arısoy, Görkem Saka, Mehmet Can Demirci, Melissa Bilec, Natasa Vidic, Mustafa Baz, Pınar Yıldırım, Murat Kurt, Korhan Turhan, Osman Özaltın, Erin Claypool, Burhaneddin Sandıkçı, Gillian Nicholls, Chen Li, Shengnan $\mathrm{Wu}$, Oscar, and Guiping for their support and friendship. I would like to express my gratitude especially to Halil Bayrak and J.P. Lai for their invaluable input in my dissertation and support in many ways. Final thanks go to the cheerful group of Zeynep Erkin, Sakine Batun, Iş11 Öndeş, Gözde İçten, Anıl Yılmaz, and Alp Şekerci!

I am forever indebted to my wonderful parents, Hale and Ülgür, and to my dear sister Gökçe for believing in me and for their unconditional support and encouragement. This work would never be the same without them.

Finally, I am very grateful to my amazing wife and best friend, Şebnem, for her endless love, patience, absolute support, and for helping me keep my life in proper perspective and balance. I am very indebted to her for sharing all my troubles during hard times and for her enthusiasm that helped me overcome so many problems.

Thank you all... 


\section{NOTATION}

Sets $i, k \in I$ :

Sets $j, l \in J$ :

Set $\alpha_{i} \in \mathcal{A}_{i}$ :

Set $t \in \mathcal{T}$ :

Set $n \in \mathcal{N}$ :

$\boldsymbol{P}$ :

$S:$

$A_{i}:$

$T$ :

$N$ :

$c_{i j \alpha_{i} t}{ }^{1}, c_{i j \alpha_{i} t}{ }^{2}$
Sets of components from component 1 to component $P$

Sets of suppliers from 1 to $S$

Set of design alternatives of component $i$

Set of time periods

Set of binary factorization elements of lead time - demand multiplication

Total number of components used in the product $(|\mathcal{I}|=P)$

Total number of available suppliers $(|\mathcal{J}|=S)$

Number of design alternatives for component $i\left(\left|\mathcal{A}_{i}\right|=A_{i}\right)$

Number of time periods (each representing a product life cycle phase) $(|\mathcal{T}|=T)$

Number of lead time - demand binary variables $(\gamma$ and $\delta$ ) that cover all possible $L T_{1 t} \times$ Demand $_{1 t}$ values in a binary representation $(|\mathcal{N}|=N)$

Unit manufacturing costs of component $i$ at supplier $j$ for production levels 1 and 2 for component design $\alpha_{i}$ at time period $t$ 
Capacity $_{i j \alpha_{i} t}{ }^{1}$, Capacity $_{i j \alpha_{i}{ }^{t}}{ }^{2}$ : Total production capacity of supplier $j$ for component $i$ for production levels 1 and 2 for component design $\alpha_{i}$ at time period $t$

Netwco jl: $^{2}$

Relation $_{\text {ik: }}$ :

$p t_{i j \alpha_{i} i}$

val $_{i a_{i}}$ :

$\operatorname{Tportcost}_{\boldsymbol{j k l a}_{\boldsymbol{k}}} \boldsymbol{t}$

$\omega_{1 t}, \omega_{2 t}, \omega_{3 t}:$

$\boldsymbol{h}_{\boldsymbol{t}}$

$\boldsymbol{\beta}_{1}, \boldsymbol{\beta}_{2}:$

timemultiplier $_{t}$.

periodlength:

$z_{\text {ssratio: }}$

$\rho_{1}, \rho_{2}:$

$\operatorname{Mcap}_{\text {ia }_{i} t}$ :
Fixed supply chain network costs between suppliers $j$ and $l$

Number of components $k$ required to manufacture component $i$

Production time of design $\alpha_{i}$ of component $i$ at supplier $j$ in time period $t$

Value of design $\alpha_{i}$ of component $i$ for the demand in time period $t(\%$ of total contribution)

Unit transportation cost of design $\alpha_{k}$ of component $k$ from supplier $l$ to $j$ in period $t$

Allowed values that price can take in time period $t$

Unit inventory holding cost of the final product in time period $t$

Demand function coefficients

A parameter value in order to adjust demand value according to the time period $t$ (based on what life cycle phase $t$ is)

Length of the time period $t$ (in the same units with lead time)

Z-value from the normal distribution corresponding to the given safety stock ratio (ssratio)

Constant coefficients of variation for demand over lead time and lead time, respectively

Total available capacity for design $\alpha_{i}$ of component $i$ in time period $t$ over all suppliers $\left(\sum_{j=1}^{P}\right.$ Capacity $_{i j \alpha_{i} t}^{1}+$ Capacity $\left._{i j \alpha_{i} t}^{2}\right)$ 
Mdem:

$x_{i j \alpha_{i} t}{ }^{1}, x_{i j \alpha_{i}{ }^{t}}{ }^{2}$

price $_{t}$ :

$y_{j l}$ :

$\boldsymbol{a}_{i j \alpha_{i} t}$ :

$\boldsymbol{u}_{j k l a} \boldsymbol{k}$ :

$\pi_{i \alpha_{i} t}$ :

$v_{t}:$

Demand $_{i t}$ :

$\phi_{1 i}$ :

$\phi_{2 i}$ :

$\lambda_{1 \mathrm{t}}, \lambda_{2 \mathrm{t}}$ :

$\tau_{t}^{+}:$

$\tau_{t}^{-}$

$\boldsymbol{k}_{t}^{+}$:

$\boldsymbol{k}_{t}^{-}$:
Maximum potential demand over all periods (calculated by using maximum of timemultiplier $_{t}$ and lowest price $_{t}$ with $v_{t}=1$ )

Total production amount of component $i$ at supplier $j$ for production levels 1 and 2 for component design $\alpha_{i}$ at time period $t$

Price of the final product in time period $t$

1, if suppliers $j$ and $l$ have a direct relationship; 0 , otherwise

1, if supplier $j$ fulfills its level 1 capacity with design $\alpha_{i}$ of component $i$ in time period $t$; 0 , otherwise

Total amount of design $\alpha_{k}$ of component $k$ manufactured at supplier $l$ and transported to supplier $j$ in time period $t$

1, if design $\alpha_{i}$ of component $i$ is selected for time period $t ; 0$, otherwise

Total value of the final product design for time period $t$ (between 0 and 1 , calculated by a constraint in the model)

Total demand for component $i$ at time period $t$

1 , if price values are increased to $\omega_{2 i} ; 0$, otherwise

1 , if price values are increased to $\omega_{3 i} ; 0$, otherwise

Variables that reflects pricing decision onto demand generation via $\phi_{1}, \phi_{2 t}$, and $v$ in time period $t$

1 , if demand > total production; 0 , otherwise

1 , if total production $>$ demand; 0 , otherwise

Equal to demand, if demand > total production; 0, otherwise Equal to total production, if total production > demand; 0, otherwise 
$\psi_{1 t}, \psi_{2 t}, \psi_{3 t}, \psi_{4 t}$ : Control variables that link pricing decisions and demand or total production values for revenue calculation in time period $t$

$\boldsymbol{L} \boldsymbol{T}_{\boldsymbol{i t}}$ : $\quad$ Total lead time for component $i$ in time period $t$

LTint $_{t}$ : $\quad L T_{1 t}$ value rounded up to the nearest integer

ptime $_{i t}$ : $\quad$ Maximum production time for component $i$ in time period $t$

$\gamma_{n t}: \quad 1$, if $\mathrm{n}^{\text {th }}$ binary factor for $L T_{1 t}$ is selected for time period $t ; 0$, otherwise

$\boldsymbol{\delta}_{\mathrm{nt}}$ : $\quad$ A variable to reflect lead time-demand multiplication via binary factorization in time period $t$ 


\subsection{INTRODUCTION}

This research focuses on understanding the impacts and proposed benefits of incorporating the supply chain configuration problem into the product design phase analogous to the Design for Manufacturability (DFM) concept introduced in the 1980s where manufacturing processes of a product are taken into account in the product design phase. Academic studies and industry experiences show the benefits of incorporating different aspects of the production phase into the product design process. As the product design establishes different requirements on the manufacturability, assembly, cost, and similar parameters that have significant impacts on the later phases of the product's life cycle, supply chain is also very closely linked to product design decisions and impacted by these decisions. Therefore, the same benefits that are suggested with other Design for $\mathrm{X}$ approaches are also applicable for the Design for Supply Chain (DFSC) concept. Different Design for $\mathrm{X}$ approaches consider the impacts of the product design on various concepts such as cost, assembly, environment, and supply chain separately. However, since there would only be a single product design, its impacts on these various issues would be cumulative that is a product design may be optimized for minimum cost or easy assembly but this same design may not be the optimal decision for the environment or supply chain issues. Although in a perfect case, all of these problems would be combined and solved concurrently, each of these problems is very complex and needs to be studied in detail before being combined. In this research, the Design for Supply Chain approach is investigated to quantify and understand 
the impacts of the product design on the supply chain performance. The proposed benefits of the DFSC are investigated by utilizing a mathematical programming approach in order to quantify these proposed benefits.

The developed mathematical programming model is structured in a way to optimize product design and supply chain decisions with an aim of maximizing profits throughout the product's life cycle. At the same time, this model allows for further investigation of the proposed benefits of incorporating supply chain decisions into the product design phase by providing the opportunity to exclude supply chain related decisions and evaluate the impacts of this exclusion. The model includes widely known product and supply chain design aspects, and has also been validated by industry experts. This ensures that the model captures important design parameters that are being used in the industry and is as realistic as possible.

While this model is designed to be very realistic by considering primary product design and supply chain performance parameters, it tends to be mathematically very complex and hard to solve. Therefore, this research also aims to evaluate different solution techniques such as deterministic optimization procedures, heuristics, and their combinations in order to find optimal or near-optimal solutions quickly.

\subsection{MOTIVATION}

Research studies demonstrate that the average discrete manufacturer realizes a $12 \%$ reduction in time-to-value, a 20\% reduction in development costs, and a $7 \%$ reduction in manufacturing costs by collaborating with the supply chain early in the design process [32]. Most benefits of collaboration among supply chain (SC) partners lie in the design phase of the product lifecycle, 
since the cost of design changes increases as the design phase of the product life cycle ends and the manufacturing phase starts. It has been reported that at least $70 \%$ of the product costs become firm in the design phase [3]. Keys [34] reported that some $75-90 \%$ of opportunity to influence total life cycle cost is gone by the time a design is released to production. Figure 1 depicts the percentage of costs committed throughout the product design process.

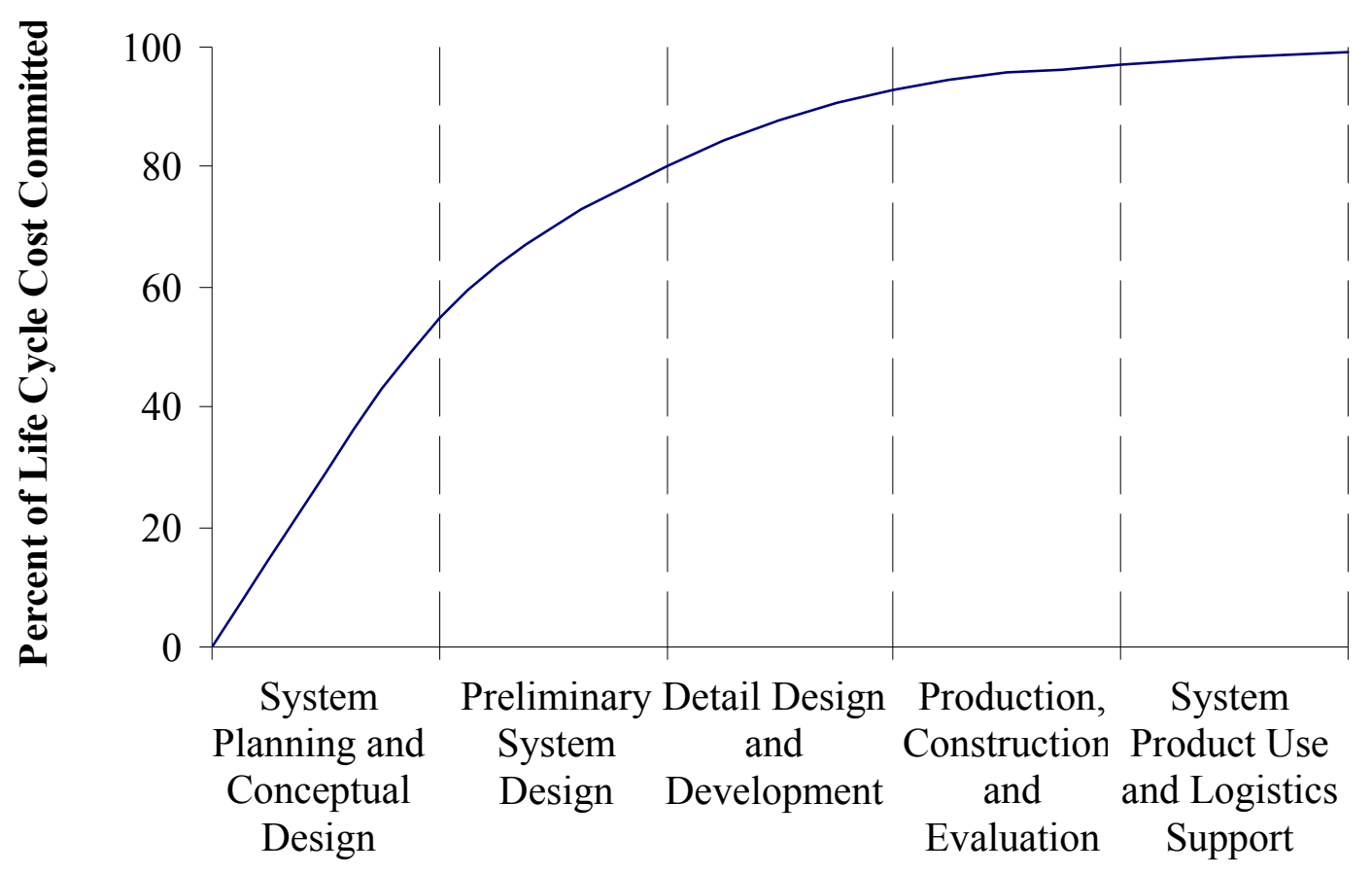

Figure 1: Actions affecting life cycle cost (adapted from [34])

Different aspects of the supply chain such as transportation planning, warehouse selection, and supplier selection contribute to the total supply chain performance and should be planned according to the product design and marketing targets. These aspects also constitute a large portion of the product cost after the design phase and they are significant sources of cost as standalone operations. For example, in the U.S, annual expenditures on non-military logistics 
represented over $11 \%$ of the Gross National Product in 1995 [55]. A decade later in 2005, the total logistics costs total around $\$ 1.3$ trillion, still representing $10 \%$ of the Gross National Product [19]. Therefore, the proposed benefits of designing the supply chain within the product design or redesign lifecycle phases present huge impacts on both the cost and other performance measures of the supply chain and success of the product. In this research, a product's success is defined as its ability to generate enough demand and to satisfy this demand via the associated supply chain during its life cycle period. For example, a product could be considered as successful if its life cycle spans as long as planned, if it attracts targeted demand at the targeted markets, if it adds value to the company and its brand, and if it satisfies customers, therefore generating expected profits.

This research aims to investigate the impacts and proposed benefits of incorporating the supply chain configuration problem into the product design phase. The motivation behind the study is that proposed benefits could be understood and considered by managers when conceptual ideas are supported with case studies to include quantitative analysis and performance metrics. This research investigates the formulation of advanced and complex product design and supply chain models with the investigation of several solution techniques to solve these models in a timely manner with optimal or near-optimal results. Here, product design models aim to solve product design selection, pricing, and similar problems; where supply chain models are mainly developed to design the supply chain by selecting suppliers and setting the relations between them. 


\subsection{PROBLEM STATEMENT}

In the complex business environment of the current technology era, it is recognized that not paying enough attention to product design might potentially result in financial losses due to demand generation problems. In this context, demand generation is used to define how attractive a product design is in terms of creating demand. In other words, it is the ability of a product design to generate demand by satisfying customer expectations. As the supply and therefore the competition among the companies for the limited market demand increases, the product design gains more importance for demand generation. However, the product design impacts not only the demand generation, but also the manufacturing processes, cost, quality, and lead time. The product design affects the associated supply chain directly with its requirements including, but not limited to manufacturing, transportation, quality, quantity, production schedule, material selection, production technologies, production policies, regulations, and laws. From a broad perspective, the success of the supply chain depends on the product design and the capabilities of the supply chain, but the reverse is also true, the success of the product depends on the supply chain which produces it. Since the product design dictates multiple requirements on the supply chain as mentioned previously, it is clear that once a product design is completed, it would determine the structure of the supply chain, limiting the flexibility of the engineers to generate and evaluate different supply chain alternatives. Furthermore, constructing an optimal supply chain is a key for the success of the product because of its impacts to cost, quality, and schedule. In this research, the impacts of the product design and redesign on the supply chain structure is studied with an aim at quantifying those impacts so that they can be used in the product design phase to better understand the tradeoffs between the benefits and costs of the different supply chain alternatives. The problem that the product design engineers and managers face in the 
product design phase is to pick the "best" design among several alternatives in order to generate the targeted profits or benefits. This "best" design is decided generally based on its potential for demand generation in the industry. Thus the "best" product design would be the design which satisfies the customer requirements at the utmost level. Therefore, there is always an option to select the "best" possible product design and then optimize the supply chain for this fixed product design. In this research context, from the total profit point of view, the "best" product design would be the one that sacrifices (if necessary) some demand attractiveness for better supply chain performance so that the total profits (as a combination of both generated demand and supply chain performance) are maximized. Since the design decisions generate many constraints regarding manufacturing and supply chain requirements, the design alternatives need to be studied and compared considering their impacts to the supply chains. Broadly speaking, in addition to "always selecting the best possible design" approach (without regard to the supply chain design), there are two different approaches for product and supply chain design integration namely sequential and simultaneous as depicted in Figure 2.

The sequential approach, which is widely applied in industry, suggests that product design should be completed based on customer, marketing, and management requirements independently from analyzing the supply chain impacts. After the design is completed, the supply chain should be generated and then examined for its performance. If the supply chain performance is not within desirable limits, the product design should be revised until a satisfactory supply chain performance is achieved. However, as it will be further explained and studied in Chapter 3.0, this approach very likely leads to a suboptimal product and supply chain design configuration due to solving each problem separately. Even after an optimal product design is created for customer and company requirements, subsequent changes to the component 
and part designs to achieve a better performing supply chain may lead to a non-optimal product and supply chain design.

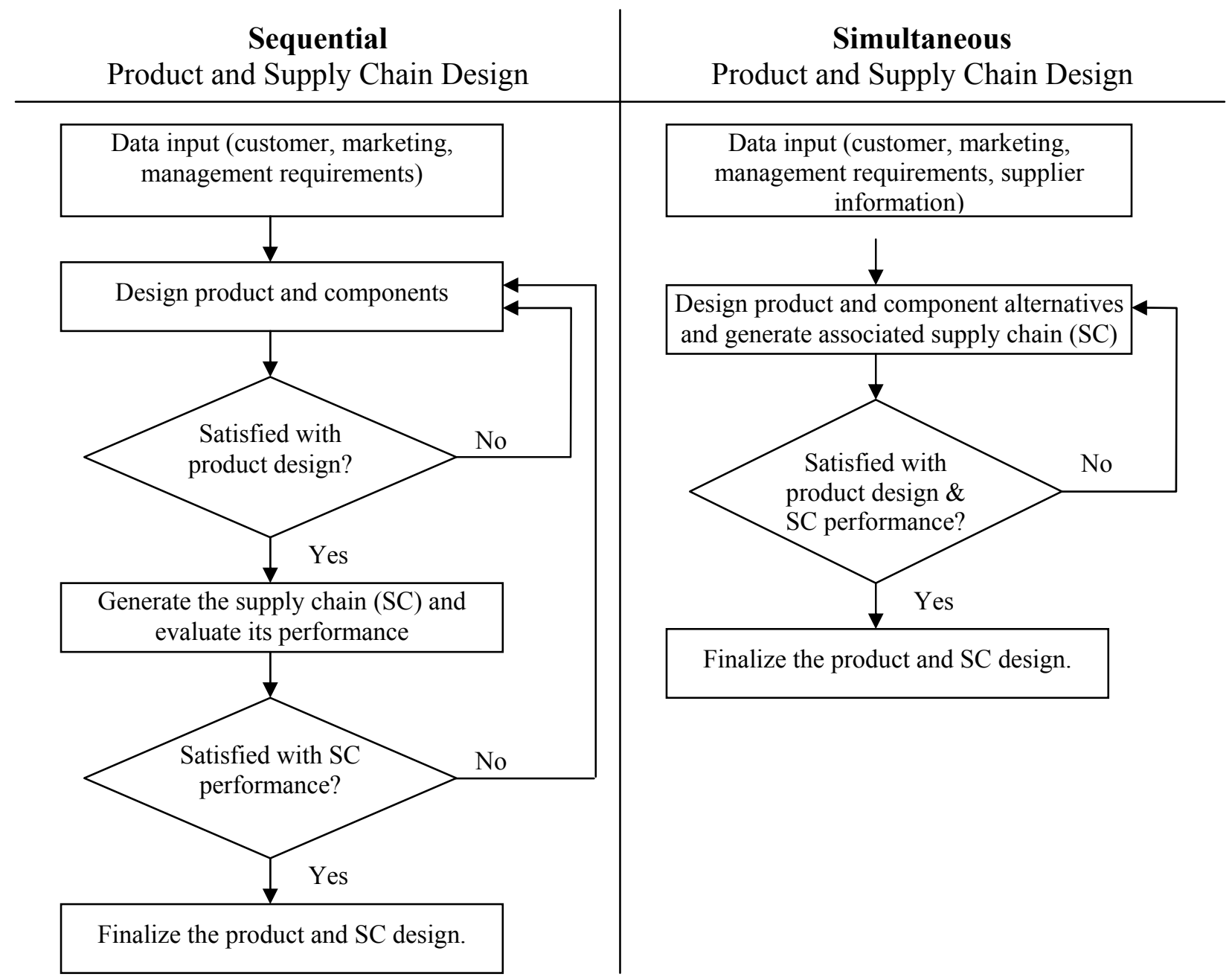

Figure 2: Sequential and simultaneous product and supply chain design processes

In contrast, a simultaneous product and supply chain design would overcome these shortcomings. The proposed approach uses not only customer, marketing, and management requirements necessary for the product design, but it also incorporates supplier information. With this approach, the cyclic procedure of designing a product, generating the supply chain, 
evaluating the supply chain, and redesigning the product is reduced in many cases to a single iteration. However, the costs and time requirements associated with determining or designing several components at once need to be considered for the simultaneous approach. The main tradeoff between the sequential and simultaneous approach would be designing several component alternatives at once for the simultaneous approach versus launching a non-optimal product design - supply chain combination which might lead to lower profits with the sequential approach.

The problem addressed in this research is about picking the "optimal" product design by evaluating its impacts on the demand generation and supply chain network requirements. Briefly, developed models evaluate and compare the product design alternatives to select the optimal one which maximizes the company's profit by generating more demand and supplying this demand with high quality products in a timely manner and with less cost.

\subsection{RESEARCH QUESTIONS}

This research investigates the development of a process for Design for Supply Chain [52] - a process that aims to drastically reduce the product life cycle costs (including design, production, and logistic costs), improve product quality, improve efficiency, and improve profitability for all partners in the supply chain. It presents supply chain models aimed at economically managing the supply chain for product design and supports not only product design, but also redesign associated with process improvements and design changes in general. It suggests development of a proactive approach to product design and design change allowing impacts to the supply chain to be predicted in advance and resolved more quickly and economically. 
A typical example of DFSC is the automotive OEM with multi-tier suppliers and corresponding product data and information transfers to facilitate supply chain profitability. OEM's can focus on core competencies and optimize intellectual assets while outsourcing processes that are integral to their finished products [8]. Another objective of DFSC is to elevate the supply chain to the level of a design and development partner to create a ripple effect of benefits consistent with an OEM's primary objectives - quality and time to market [1]. It would also help to eliminate the "bullwhip effect" [48] (cascading rise in inventory and financial bottlenecks due to unanticipated changes to product design).

By utilizing mathematical programming and heuristics on the DFSC models, this research specifically aims to answer four major research questions by studying several related subquestions:

\section{Which product design / supply chain performance metrics should be included in the model?}

This question inquires which performance metrics should be considered in the model since each additional metric would increase the complexity of the model. This research investigates impacts of different performance metrics on the complexity and quality of the solution techniques. Benefits and costs of adding new metrics to the model are analyzed.

1.1. How much computational complexity does a performance metric add to the model?

1.2. Are there specific performance metrics (such as cost and lead time) that should always be considered in the model?

1.3. How does the performance of the solution techniques differ by adding another performance metric to the model? 


\section{How do the performance metrics differ for the product design and the associated supply chain for the simultaneous and sequential approaches?}

This question asks whether the simultaneous approach would impact the final product design and supply chain configuration that would have been selected by the sequential approach. It also examines which supply chain performance metrics would differ by using the simultaneous approach and whether any of these metrics would be improved.

2.1. Does the simultaneous approach provide a less costly product design and supply chain through the product's life cycle?

2.2. Does the simultaneous approach create a supply chain that has minimum lead time?

2.3. To what level does the simultaneous approach modify the product design that is optimized for customer preferences?

2.4. Does the non-optimal product design (according to customer preferences) pay for itself through better supply chain performance?

\section{How robust is the supply chain to product design changes?}

The underlying idea is that there would not be any significant differences between different supply chains for a given product design. This question is explored by studying different supply chain configurations for a given product design and measuring both their forecasted and realized long-term performances.

3.1. Do product design changes result in a change to the number of suppliers?

3.2. Do product design changes result in a change in the number of tiers in the supply chain?

3.3. Do product design changes result in a change of the actual suppliers?

3.4. How sensitive is the supply chain to product design changes, i.e., what level of magnitude of changes result in a change to the supply chain? 
3.5. Does the simultaneous design approach provide a more robust supply chain structure than the sequential approach?

\section{What is the relative importance of the product design and the supply chain design on the product success (thus on the profits)?}

This idea questions whether the design team of the product should only concentrate on the demand generation potential of the product design rather than considering its impacts on the supply chain, assuming effects of the supply chain performance on demand generation is negligible when compared to those of the product design itself. This question is studied by comparing the impacts of the product design and the supply chain performance on profit.

4.1. How does optimal product design differ when supply chain structure is considered in addition to customer preferences than when it is not considered?

4.2. How much impact does the product design and supply chain have on the product's success (profits)?

\subsection{CONTRIBUTION}

This research makes several significant contributions. First, a significant addition to the existing DFSC research is provided by combining the product design and supply chain decisions into a single framework which optimizes the decisions simultaneously. This research aims to fill in the gap in the DFSC literature which lacks explicit consideration and integration of marketing and product design decisions and manufacturing and supply chain decisions. The previous academic studies concentrated on the integration of the product differentiation point or product Bill of 
Materials requirements to the supply chain optimization models, and hence lack any assessment of impacts of these decisions on the customer demand and satisfaction. This research uniquely addresses both the demand and manufacturing aspects of a product and it employs wellestablished product design and supply chain performance metrics.

The second contribution of this dissertation is that the developed models are not limited to certain industries or products but rather they are generic and can be used virtually for any type of product in any manufacturing industry.

The third contribution of this dissertation is that it investigates and provides insight about possible solution procedures, including Mixed Integer Programming and Genetic Algorithm, and their benefits to the solving these complex DFSC models. Detailed computational tests and illustrative examples provide a clear assessment of both solution methodologies. In addition, this dissertation evaluates alternative modeling preferences and assesses the performance of different solution methodologies for these alternative models. Furthermore, the presented analyses provide insight into the modeling and algorithmic / computation complexity issues.

Finally, this dissertation provides an important assessment from different industry experts across various industries and includes these experts' validation and suggestions for further work.

\subsection{OVERVIEW OF THE DISSERTATION}

In this dissertation, Chapter 2 provides a summary of the relevant literature that is related to product and supply chain decision problems. The first section in this chapter describes separate product design and supply chain design studies followed by an explanation of the research that investigated a combination of product and supply chain design similar to the DFSC approach. 
The following sections concentrate on mathematical model formulations and solution techniques used for these separate and combined problems. A summary of the literature is provided in the last section.

The mathematical model formulation is given in Chapter 3. The detailed problem description in the first section is followed by preliminary mathematical models in the second section. The third and fourth sections describe the complete DFSC model formulation and reduced DFSC models used to investigate research questions, respectively. The last section provides a model asymptotic size analysis for the described models.

Brief history and capabilities of deterministic optimization methods and relevant heuristics are given in the first and second sections of Chapter 4. This chapter also provides a comparison of these methods' performance on the preliminary models in the relevant subsections. Finally, a complexity analysis of these solution methods is provided.

Chapter 5 concentrates on the computational results first by analyzing how different solution techniques perform for the described models. The second section is the problem and model validation and it includes the industry experts' assessments, comments, and revisions about the significance of the problem, the mathematical model structure and assumptions, and finally the computational results. The last section in this chapter addresses research questions and provides answers for these questions based on the computational results.

Chapter 6, the last chapter, presents a summary of the findings and concluding remarks. The second section provides future work both in extending the DFSC models and studying other solution techniques. 


\subsection{LITERATURE REVIEW}

Examination of the scholarly literature shows growing interest in supply chain problems and the relationships between the product design and supply chains as the potential impacts of supply chain decisions become more tractable, understandable, and easy to evaluate. Since the World War II era, there has been a growing interest in supply chain planning to increase efficiency and performance of supply chains. At the same time, product design and customer satisfaction interactions have gained increasing interest by researchers working in both design engineering and marketing. With the advancements in problem modeling and solution techniques, limited supply chain and product design problems are extended to capture more realistic aspects of these problems. In the last two decades, combining these two important and interacting problems continues to draw attention both by academia and industry.

This literature review provides a summary of these efforts that are aimed at modeling and solving product design and customer satisfaction problems, supply chain problems, and their combinations. The literature review section is organized into three sections, namely problem types (Section 2.1), model formulation (Section 2.2), and solution methodologies (Section 2.3). Although several studies consider modeling, solution, or problem aspects collectively, they are presented within the literature survey according to their main contributions. 


\subsection{PROBLEM TYPES}

As this research examines a combination of product design and supply chain design problems, these two separate problems need to be comprehended. The product design problems often tend to be studied within the marketing and industrial engineering domains as they relate to the demand generation context of this research. On the other hand, supply chain problems are more widely studied within the industrial engineering optimization area although the majority of the research still concentrates on logistics aspects. Relevant studies to these different problem types are presented.

\subsubsection{Product Design Models}

The product design, along with price, advertising, and other market variables, is always considered one of the most important variables that impact demand. As each product is sold to satisfy certain customer needs, the product design addresses these needs by providing different features via different components. Each different component design satisfies these customer needs to a different degree. For example, car tires mainly satisfy customers' needs for good road traction and a smooth ride. However, different tire thread designs such as dry or snow road threads fully satisfy these needs in the corresponding road conditions. Therefore, using a dry road thread on a car to be sold in a market with many snowy days would satisfy customers' needs partially, resulting in lower demand.

As an important effort in conceptualizing the interaction between the product design and satisfaction of customers' needs, the Kano model, given in Figure 3, examines degree of 
achievement by the product design against the customer satisfaction. It defines three major areas of customer satisfaction and their relationship to products features.

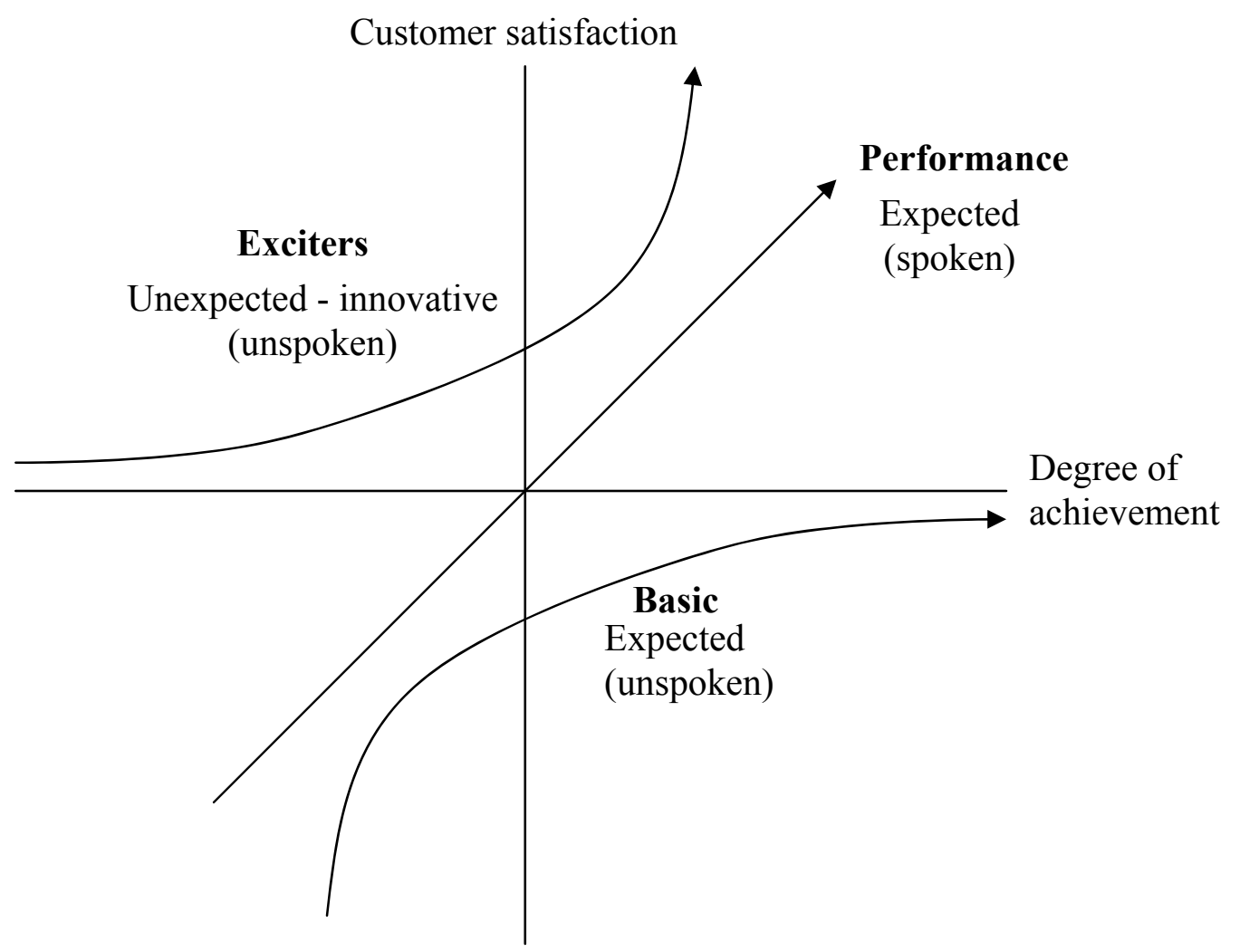

Figure 3: The Kano model (adapted from [9] and [29])

According to the Kano model, the straight line represents expected performance measures from a product design. These expectations are usually expressed by the customers so the designers know what they need to provide as product features. The exciters or innovative design features are not expected by the customers and therefore are not expressed. Thus, if they are not achieved, they do not result in loss of customer satisfaction, however when they are provided, they provide extraordinary positive impact on customer satisfaction. On the opposite, basic design features are not expressed by the customers even though they are expected from a product design. Safety and 
reliability features are usually in this category. Satisfaction of these basic expectations normally do not result in customer satisfaction, instead they only prevent customer dissatisfaction.

In order to quantify the relationship between product design and satisfaction of customers' needs, various models have been studied in the literature. In the robust design process context, Taguchi developed a "quality loss function" that evaluates the level of the dissatisfaction of customers' needs (and value) when a product component's design is different than its ideal specifications [21]. Although Taguchi developed more than 68 loss functions, the most widely used function, which is called nominal-the-best type given in (1.1), quantifies loss of customer value when a component design, $y$, varies from its targeted ideal value, $\tau$. The quality loss coefficient, $k$, is assumed or determined by an expert and depends on the product or market and is defined in monetary values [9].

$$
L=k(y-\tau)^{2}
$$

Another important approach is Value Engineering (VE) which was developed at General Electric during World War II. In this approach, the value of a component (and consequently its design) is defined as the ratio of its function to its cost. Although VE does not necessarily evaluate the satisfaction of customers' needs by the product component's design, it is widely used in industry to eliminate non-value added costs in product designs [17][18] and it is often required by the U.S. Department of Defense and NASA of their contractors [5].

Quality Function Deployment (QFD), which was developed by Dr. Shigeru Mizuno in 1972 and first applied in Mitsubishi in Japan, is "a planning tool to fulfill customer expectations" [9]. QFD is widely used to make design tradeoff decisions between component alternatives [17]. In this method, customers' needs (WHATs) are transformed into design specifications (HOWs) by design engineers in the first step. Next, the relationships between customers' needs and design 
specifications are determined. Following these assessments, competing products' specifications are also added into the relationship matrix, representing strengths and weaknesses of the proposed product design alternatives. In later steps, customers' priorities on these features and associated technical difficulties to manufacture these designs are assessed. In conclusion, QFD provides a structured method to evaluate the satisfaction level of customers' needs via features proposed by the product design alternatives. An extensive literature survey of Chan and $\mathrm{Wu}$ [12] suggests that QFD is widely used to collect and translate and to satisfy customers' needs.

As another effort to quantify the customer satisfaction and the product design interactions, Martin and Ishii [47] studied impacts of design variety on the customer satisfaction and manufacturing processes. In parallel to the QFD method, they developed an index called Variety Voice of the Customer $\left(\mathrm{V}^{2} \mathrm{OC}\right)$, which is a measure of the importance of the component to the customer, as well as the heterogeneity of the market with respect to that component. In this method, if a component is ranked with low $\mathrm{V}^{2} \mathrm{OC}$ value, then it is suggested that this component design is standardized for cost reduction. However, a high $\mathrm{V}^{2} \mathrm{OC}$ rating suggests that the corresponding component is of critical value for customer satisfaction and therefore should be designed to satisfy different customer requirements.

Although there is an extensive literature of product design and its impacts on customer satisfaction (and consequently demand generation), a brief overview of important studies and concepts are presented. These studies suggest different methods can be employed to map customers' needs to design specifications. They also suggest that various methods can be used to quantify impacts of satisfaction of customers' needs on the demand. However, despite the existence of different methods, it should be noted that the product design and demand generation 
relationship is an important issue and it needs to be quantified in a structured way to the extent possible to represent the complex interaction.

\subsubsection{Supply Chain Models}

A supply chain, which is defined as "consisting of all parties involved, directly or indirectly, in fulfilling a customer request" [13], is a continuously evolving part of a company's production process and plays a critical role in the profitability of a product and the company. A supply chain starts with the raw material procurement and includes all tiers of suppliers that manufacture product components, production facilities that utilize manufacturing and assembly operations, warehouses and distribution centers, retailers, customers, and post-sales service centers. In this research, the supply chain configuration problem focuses on a segment of the supply chain beginning with the raw material procurement and ending with the final product manufacturing or assembly. Chopra and Meindl [13] suggest that toward the customer's request fulfillment objective, a supply chain not only includes manufacturing and transportation of products but also consists of marketing, new product development, distribution, finance, and customer service. Within this definition, a supply chain is considered a vital part of a product's life cycle which starts with customers' needs and ends only after the last customer service is completed, representing a time horizon longer than the actual selling period of a product. Obviously, each of many parts that constitutes a supply chain brings important planning challenges and these problems are widely studied by academicians.

From an academic perspective, interest in supply chain problems has begun almost with the first operations research studies in the World War II era that examined transportation, scheduling, and facility location problems. As one of the earliest studies, in 1959, Hanssmann 
[31] modeled material procurement, production, and distribution with an aim of optimizing the level of inventory and selecting optimal inventory locations. In 1960, Clark and Scarf [14] developed a model to optimize purchasing decisions from different echelons of a supply chain, each having a different level of available inventory and different lead times. The supply chain has gained more attention from academic and industry beginning in the mid 1970's by studying different solution techniques for supply chain problems as explained in section 2.3. In the 1980's, several studies concentrated on inventory optimization and economic order quantity determination problems [55]. These, mainly single vendor and single buyer models are extended to capture multi-vendor, multi-buyer, and multi-period cases in the 1990's [55]. However until the mid 1990's, supply chain models often concentrated on single subproblems, such as inventory planning and buyer-vendor coordination. Later studies are aimed toward combining these subproblems into larger but more realistic complete supply chain models. For example, Cohen and Lee [16] formulated their model beginning with the material procurement, then production, and concluding with the product distribution portions of the supply chain and used a series of linked, approximate submodels, and heuristics optimization to solve the problem. They introduced stochastic demand and lead time parameters into the model and suggested different heuristic solution procedures for material control, production, stockpile inventory, and distribution submodels that constitutes the final supply chain model when combined.

In parallel to these operational supply chain problems, strategic supply chain planning and supply chain network design problems are also widely studied within the engineering and business literatures. One of the initial papers that proposed a model and an efficient solution technique for specific kinds of supply chain problems was dated back to 1974 in which the authors modeled a static distribution system to optimize the locations of intermediate distribution 
facilities between plants and customers [24]. This paper reports significant computational time gains by employing Bender's Decomposition technique and having solutions within one or two tenths of one percent of the global optimum. Viswanadham and Raghavan [60] investigated performance of different supply chain networks under two different production planning and control policies, namely make-to-stock and assemble-to-order systems. Truong and Azadivar [56] developed a model to design the network of a supply chain that addresses supplier selection, distribution center location, inventory planning, and production orders. Cakravista et al. [10] developed a two-stage supply chain planning model which performs supplier selection based on supplier bids and determines optimal manufacturing and logistics functions. Talluri and Baker [54] developed a multi-phase model that evaluates each supplier's production efficiency and incorporates this analysis into supply chain network design problem. Their model also optimizes production assignments to selected suppliers. Wu and O'Grady [64] developed a methodology to improve design of a supply chain network for multi-criteria in a constrained environment. Ding et al. [20] developed a supplier selection model that incorporates uncertainties emerging from demand, production, and distribution parts of a supply chain. Vidal and Goetschalckx [58] presented a mathematical model that maximizes the after tax profit of a multinational company by using transfer prices (prices a company's different departments charge for semi-products within the company) and the allocation of transportation costs as explicit decision variables. This selection of studies represent the wide spectrum of the challenges that are directly associated with the supply chain problems in today's global business environment.

Thomas and Griffin [55] surveyed different supply chain models at operational and strategic levels both in academic and business literatures and stressed the lack of life cycle and inventory obsolescence constraints in the supply chain models. They stated that these problems 
can partially be attributed to the supply chain structure and design. They also reported the importance of adding new objectives into the models due to current business trends. For example, they suggest including environmental regulations, and incorporating important macroeconomic parameters, such as currency exchange rates, which becomes more important with the movement towards globalization and outsourcing. Lambert and Cooper [36] stress that in today's supply chain management problems; marketing decisions also play a significant role. Customer relationship management, demand management, and product development and commercialization are all vital parts of the supply chain management problems. Additionally, in parallel to the globalization, supply chain problems become far more complicated than classical logistics problems with the integration of long-term strategic supplier relations and marketing aspects.

These selected studies represent emerging interests from more than six decades of supply chain research. As the trend shows, the current interests in supply chain research are more focused on developing complicated, thus realistic, models as conceptual combinations of previously studied sub-models with the addition of emerging industry needs. Similarly development of different solution techniques that can provide good solutions in acceptable time for larger models has also gained more importance as realistic models challenge existing, wellestablished solution techniques currently in use.

\subsubsection{Product Design and Supply Chain Combined Models}

Although supply chain management and decision models are widely studied in the literature, the

Design for Supply Chain concept is specifically addressed in several studies. The term Design 
for Supply Chain Management (DFSCM) is first used by Lee and Billington [41] in an inventory planning problem within a supply chain review study. The authors stated that

A lot has been written on design for manufacturability, for assembly, for quality, for producibility, and for serviceability. To this list we would add "design for supply chain management." Thus product designs should be evaluated not only on functionality and performance but also on the resulting costs and service implications that they would have throughout the product's supply chain. The same applied to process designs. [[41], p.11]

In another study, Lee [40] suggested that changing the mind-set of the design teams and quantification of benefits of the DFSCM are the most important and significant obstacles for implementation.

Keys [34] discussed the Design for Life Cycle concept that he described as the combination of all design efforts including but not limited to manufacturability, assembly, cost, quality, customer support logistics, and supply chain. He suggested that it is very important to keep the focus on the product life cycle since today "the planning of new products includes second, third, etc., generation product follow-on's, where the support to and through product lives must be a part of an integrated life cycle strategy."

In a study by Lee and Sasser [44], Hewlett-Packard's (HP) new product line, called Rainbow, was used to study an analytical DFSC model which incorporates stochastic demand with lead time decisions, service level targets, and inventory, stockout, and shipment costs in order to decide on the optimal product differentiation point. The authors provided analytical solutions for the model for different product life cycle phases as well as for different design alternatives. The importance of the study is that it was done using real data and the results were validated after the product launch over the product life cycle. Another important contribution is that all the modeling and analysis was done during the product design phase, which differs from the majority of supply chain related studies that try to optimize the supply chain for products past 
the design phase. Although these authors studied a supply chain configuration problem, they did not incorporate supplier selection and thus associated uncertain quality, lead time, and capacity problems. In another study completed at HP, Lee and Billington [42] reported HP's new approach to modeling and optimizing its SC policies which adds distribution, market, and product specifications into the available inventory modeling efforts. They explained the positive impacts of the new approach which suggests incorporating all related divisions of the company, key suppliers, and key customers into the SC design decisions and developing product-based supply chains. They listed four key company requirements as the basis for their model: “(1) benchmarking inventory and service tradeoffs; (2) assessing the impact of uncertainties on operational performance; (3) analyzing what-if questions for different scenarios and operating characteristics; and (4) evaluating product design impacts on the supply chain, that is, predicting how the supply chain would perform under different product and process design alternatives" ([42], pp. 47). Lee, in his several studies, introduced and used similar terms that constitute parts of the DFSCM concept. Design for Localization (or interchangeably Design for Customization [43]) concentrates on delaying the customization of a product's design for different local market segments. In relation, the Design for Flexible Manufacture [39] concept is introduced that aims to increase part commonality and interchangeable subassemblies to help reduce inventory costs and to ease supplier management. Finally, Design for Logistics [39] aims to design products so that they can be transported easily in a cost effective manner. However, all these concepts that add up to the DFSCM concept introduced by Lee and his colleagues, mainly focus on delaying product customization to reduce inventory and designing products for effective packaging and transportation. In these studies, the product design - customer demand interactions were not included, hence customer demand - supply chain performance relations were not evaluated. 
Another extensive DFSC study has been conducted by Arntzen et al. [2] at Digital Equipment Corporation (DEC), that led to a term which they coined Global Supply Chain Model (GSCM). The GSCM is a large mixed-integer linear programming model (MIP) that minimizes cost or lead times (or both) for different echelons of the supply chain and time periods with requirements of meeting the estimated demand, using limited capacities, and restrictions on local content and offset trade. The cost structure of the model included fixed and variable production costs, inventory costs, and distribution costs including taxes, duties, and duty drawback. The model was developed to support product development at DEC for about 20 new products. It was used to design the supply chain during the product design phase and reported savings of US \$1.4 billion in four years with around a 500\% productivity increase. However, the GSCM uses a combined objective function (weighted average of cost and lead time) and the objective function is limited to these two objectives. Furthermore, the fixed demand assumption is one of the major drawbacks of the model. The solution technique is a branch and bound algorithm with introduction of penalties for violating constraints to save computation time. The authors reported around a one minute solution time for problems with 2,000 to 6,000 constraints and 5,000 to 20,000 variables, with a few hundred of these variables being binary.

The impacts of different product life cycle phases and the type of product on the structure of the associated supply chain are reported to be unavoidable and necessary factors to be included in the modeling efforts in the literature. Fisher [23] stressed two distinct types of products, namely functional and innovative; and two related supply chain functions, physical (transforming raw material to an end product and concentrating mainly on cost reduction) and market mediation (ensuring the variety of products reaching the marketplace matches what consumers want to buy and concentrating mainly on lead time reduction). He gives examples of 
Campbell's Soup as a functional product with predictable demand (so use a physical supply chain with cost reducing strategy) and Sport Obermeyer's line as an innovative product with unpredictable demand (so use market mediation supply chain with uncertainty reduction techniques, such as getting orders earlier).

Wang et al. [61] suggested three types of products: innovative, functional, and hybrid and three categories of supply chains: lean supply chain (that focuses on lean manufacturing and cost reductions), agile supply chain (that aims to provide flexible and fast manufacturing to reduce lead time), and hybrid supply chain (that aims to capture both aspects of lean and agile supply chains). They used Analytic Hierarchy Process (AHP) and linear programming (LP) in a supplier selection model for solution procedures where suppliers are ranked with AHP and selected by using LP and preemptive goal programming in order to develop the desired supply chain (lean, agile, or hybrid) for the given product type (innovative, functional, or hybrid). The supply chain performance is measured by using the Supply-Chain Operations Reference model (SCOR), which is "a process reference model that has been developed and endorsed by the Supply-Chain Council as the cross-industry standard diagnostic tool for supply-chain management" [53]. Although this approach does not require extensive computational resources, the necessity of expert knowledge for AHP, setting goals and SCOR evaluations are major implementation drawbacks. Yet, expanding this solution technique as a multi-objective solution procedure which considers several objectives at the same time both in AHP and goal programming phases would be challenging, yet necessary for a better supplier selection model.

Fandel and Stammen [22] developed a mathematical model to fix the product program and the extended supply chain network. In this study, they modeled investment decisions on product development projects between alternative products to compare product life cycles with 
their development and recycling strategies. Their model evaluated impacts of these decisions on the supply chain network and aimed to develop the optimal product development and supply chain network strategy to maximize overall profits. Although their model includes recycling performance of the supply chain and allows selection of different product alternatives, they did not explicitly consider the impacts of the product design on customer satisfaction and demand. Moreover, due to extensive size of the developed model, they did not provide an efficient solution methodology for large, real life problems.

Graves and Willems [30] developed a supply chain network model for a new product that selects suppliers and assigns production orders. However, this model assumes that the product design is finalized. Despite this drawback, this model provides opportunities by accounting for time-to-market costs and allowing multiple sourcing of a product component.

Lamothe et al. [37] studied a product family selection and supply chain network design problem, similar to the proposed DFSC concept. In their study, the authors suggested a Generic Bill of Materials (G-BOM), which is a flexible Bill of Materials in terms of satisfying customers' needs. In this study, different product family variants are associated with different market segments. A market segment is considered to be satisfied by at least the corresponding product variant or by a better product variant. Within this demand satisfaction perspective, the model aimed at minimizing total supply chain costs that come from variable manufacturing, transportation, and inventory decisions and fixed facility opening and transpiration line opening costs. Although the study models selection of better product variants for satisfaction of better market segments, it does not take the impacts of product design on the demand and market share into account. With an aim of cost minimization, the authors did not include the demand generation problem associated with the product design and pricing decisions. They have also 
excluded economies of scale and international taxes and duties for simplification purposes. This study is an example of the growing interest in academia for modeling product design and supply chain interactions. Although this study precisely models product design and supply chain relations, its main drawback is the lack of explicit modeling of the impacts of product design on the product demand. Since the changing product demand may require different supply chains, overlooking the product design - demand interaction may result in a non-optimal supply chain network. Authors, in this study, also used an open-close decision structure to select suppliers and incur associated fixed costs which limits the model to capture the increasing nature of these costs with higher levels of interactions between supplier pairs.

\subsection{MODEL FORMULATION}

During the development of supply chain modeling efforts, academicians expanded the problem formulations so that initially ignored issues that complicate the problem are added for more realistic problem formulations. Yet, mathematical problem formulation is still a challenge for researchers since formulation is a primary driver of solution techniques and ultimately the complexity of the problem and solution time as well. Incorporating stochastic, nonlinear, or qualitative objectives, parameters, and variables into the formulation helps to develop more realistic models although it is usually harder to solve these formulations computationally and often requires long computational time or sacrifices from solution quality. The formulation is also closely linked to the problem studied since it describes what decisions will be made and what constraints will be considered. 
Beamon [7] provides a detailed survey of research studies in multi-stage supply chain modeling and divides supply chain models into four categories: (1) deterministic analytical, (2) stochastic analytical, (3) economic, and (4) simulation models. She also defines performance measures for supply chains in two groups: (1) qualitative (customer satisfaction, flexibility, information and material flow integration, effective risk management, and supplier performance), and (2) quantitative (a) based on cost (cost minimization, sales maximization, profit maximization inventory investment minimization, and return on investment maximization), and (b) based on customer responsiveness (fill rate maximization, product lateness minimization, customer response time minimization, lead time minimization, and function duplication minimization). She also provides a list of frequently considered supply chain model variables which includes product / distribution scheduling, inventory levels, number of stages (echelons), distribution center assignments to customer, product assignment to plants, relationships between suppliers and buyers, product differentiation step specification, and number of product types held in inventory. She stresses the importance of using multi-objectives for realistic supply chain modeling and also including demand variance and related bullwhip effect problems. Although it is a very detailed paper about supply chain modeling aspects, it does not specify any solution techniques or discuss the implications of the model parameters on solution techniques.

Viswanadham and Raghavan [60] pointed out that a portion of the supply chain may operate under a push policy while the rest may operate under a pull policy and stressed that this transition point is called a decoupling point. They also suggested four different supply chain structures: (1) serial, (2) divergent, (3) convergent, and (4) network structure. They emphasized five analytical techniques for modeling supply chain problems and measuring performance 
including (1) series-parallel graphs, (2) Markov chains, (3) Petri nets, (4) queuing networks, and (5) system dynamics. They implemented a stochastic Petri net approach using a Stochastic Petri Net Package (SPNP) to calculate the total cost of inventory and backorders under Make-to-Stock (MTS) and Assemble-to-Order (ATO) policies and studied the decoupling point problem. However, their work concentrated on cost aspects of the MTO or ATO policies only and their assumption that all variables were exponentially distributed requires further research in order to observe results for different distributions as well as their impacts on other performance measures, such as lead times or production quality.

Lee and Kim [45] developed a model for multi-product, multi-period, multi-shop production and distribution to satisfy the retailer's demand in given time periods. They proposed a hybrid solution technique which combines analytic methods with a simulation modeling approach to capture the stochastic nature of operation times in the supply chain. The objective of their model is to minimize the overall cost that includes production, transportation, inventory, and shortage costs, subject to inventory and operation time constraints. They incorporated machining and distribution time parameters from simulation results into the analytical model and proposed an iterative solution approach. This problem formulation only focused on the production and subsequent distribution questions without considering the front-end portion of the process namely raw material and vendor inputs. Despite the use of simulation, they also did not include stochastic demand aspects and removed backlog approaches which are often necessary for realistic supply chain models. In a related effort, Kim et al. [35] proposed a nonlinear model that optimizes production quantities for the final product and its components that are outsourced to different suppliers. They included capacity constraints for the manufacturer and suppliers and used a probabilistic demand function. The nonlinear model is transformed into two linear models 
by using Karush-Kuhn Tucker (KKT) conditions, thus an iterative approach is developed to find the "global" optimum. The objective function of maximizing profits is optimized for expected demand values and considered for a single period in which both the manufacturing and sales take place. The lead time and delivery delays are ignored and all the production and assembly processes are assumed to be in a single period. Despite these drawbacks, the model and solution technique provides a good example for cost oriented manufacturing and outsourcing decision problems for existing product designs. Lamothe et al. [37] modeled their product family and supply chain network design problem as a Mixed Integer Programming problem. Their model included G-BOM, product flow, shipping, inventory, and supplier capacity constraints often requiring the use of binary variables to represent open-close type decisions. However, they stress that they avoided integer variables for lead time representation given that lead times are significantly shorter than the modeled planning time periods.

Truong and Azadivar [56] described a methodology developed for an optimal supply chain configuration and proposed a hybrid solution procedure for the problem combining heuristics and simulation. They listed several performance measures for supply chain models which are grouped into quantitative and qualitative sections. Table 1 lists these performance measures.

Wu and O'Grady [64] used a network-based approach, called Extended Trans-Nets, which represents the design of the supply chain as an abstract network with 'AND' and 'OR' nodes. They stated that this approach allows different designs of the supply chain to be modeled and analyzed. However, this approach cannot identify improvements in the design of supply chain by its nature and, similar to a system simulation, different designs should be entered by the end user or another algorithm. 
Table 1: Performance measures for supply chain management (adapted from [56])

\begin{tabular}{ll}
\hline \multicolumn{1}{c}{ Performance Measure } & \multicolumn{1}{c}{ Description } \\
Cost & $\begin{array}{l}\text { It is most widely used and cost is minimized for the } \\
\text { entire supply chain. }\end{array}$ \\
\hline Return on Investment & Maximize the ratio of net profit to capital. \\
\hline Based on responsiveness to customers & $\begin{array}{l}\text { Maximize the fraction of customer orders filled on } \\
\text { time. }\end{array}$ \\
\hline Fill rate & $\begin{array}{l}\text { Minimize the amount of time required from order } \\
\text { placement to delivery to customer. }\end{array}$ \\
\hline Customer response time & $\begin{array}{l}\text { Minimize the amount of time between promised } \\
\text { delivery time and actual delivery time. }\end{array}$ \\
\hline Product lateness & $\begin{array}{l}\text { The degree to which customers (internal or external) } \\
\text { are satisfied with the product received. }\end{array}$ \\
Cualitative performance measures satisfaction & $\begin{array}{l}\text { The degree to which the supply chain can respond to } \\
\text { random fluctuations in the demand pattern. }\end{array}$ \\
\hline Flexibility & $\begin{array}{l}\text { The degree to which effects of inherent risks that } \\
\text { come from the relationships within the supply chain } \\
\text { are minimized. }\end{array}$ \\
\hline Effective risk management
\end{tabular}

Weber et al. [62] provided a literature search with 74 papers about supply chain vendor selection problems and stated that manufacturing cost, lead time, product quality, supplier capacity, and supplier location are the criteria most often used as primary decision factors.

Although there are various kinds of supply chain models with different objectives and variables, the most common problem formulation shown in the literature aims to minimize cost under specific capacity constraints. However, in order to develop more realistic models in response to industry's needs, multi-objective formulations are required and the formulations should capture the dynamic, stochastic, and structural aspects (such as push and pull strategies) inherent in the supply chains. Recent studies also mention the introduction of more stochastic elements into the model, necessitating the use of combinations of established solution techniques or development of new ones. 


\subsection{SOLUTION METHODOLOGIES}

Although there have been rapid computational technological developments in optimization methods in the last 20 years, there remains a need for more realistic and thus complicated models demanding even more powerful and timely solution techniques. It is why this area remains one of the main interests of the academic community. The supply chain problem is specifically one of these complex problems that requires new solution procedures because of the need to combine several different subproblems, such as distribution, location and allocation, transportation, and production planning in order to address the supply chain solution in its entirety. These subproblems were modeled and solved as independent problems initially because of the lack of computational resources and extensive data gathering tools as well as industry's interest in fast solutions for smaller problems. As the military and industry needs grew, academic researchers initially worked on modeling smaller supply chain subproblems with an aim of finding optimal solutions for them as fast as possible. However, the literature trends show that handling these subproblems separately leads to suboptimal solutions for the supply chain as a whole since the interdependency between them is extremely high, making it almost impossible to combine the results to get the "one" optimal solution for the entire supply chain. The changes in the industry's priorities towards solving broader, thus more complex problems in addition to the impacts of globalization, requires more academic studies that model and solve large and complex problems which are combinations of these subproblems. The technological developments in computing power and existing knowledge about these subproblems make both modeling and solving these large problems easier and help to reach optimum results.

Supply chain problems are usually multi-objective problems with conflicting objectives since realistic problems introduce several objectives at once. Usually, all of these objectives are 
very important to construct a profitable supply chain. For example, ignoring optimization of lead times and focusing only on the production costs provide a minimum cost supply chain, but it does not ensure profitability due to potentially long lead times resulting in stockouts. Despite their advances on single objective optimization, pure mathematical optimization techniques do not seem to handle the multi-objective problems well unless some modifications are introduced into the model, such as weighing/ranking objectives or penalizing solutions for poor objective values. The heuristic solution techniques, which are based on the concept of rapidly moving the solution space towards the best objective by moving to better solutions at each step, are more widely used to solve the supply chain problems because of their reasonable computational time requirements for an acceptably good solution and their flexibility to be fit into different kinds of problems.

Vidal and Goetschalckx [59] presented an extensive literature survey of global supply chain optimization models with an emphasis on the use of MIP models and solution techniques. They suggested that although complex interactions in the supply chain can be straightforwardly captured by these models, a framework that can represent quantitative and qualitative decision variables is necessary. Unfortunately, an established and widely accepted supply chain modeling structure does not exist.

Coello [15] provides an extensive review of the use of heuristic-based optimization techniques for multi-objective problems, including weighted average of objectives, goal programming, $\varepsilon$-constraint method, Vector Evaluated Genetic Algorithm (VEGA) methodology, using genders in GA for objectives, weighted min-max approach, and multiple-objective GA. He states strengths and weaknesses of each of these approaches and suggests different problems suitable for them. However, he criticizes the crucial implementation of weighing solutions to 
combine them into a single objective for pure mathematical optimization because of its drawbacks including the requirement of determining proper weights and lack of generating proper Pareto optimal solutions in the presence of non-convex search spaces. The stochastic nature of the supply chain which is a result of uncertainty of demand, quality, production and transportation times, and long term planning requirements, is also identified in the literature as a barrier for optimizing objectives with deterministic parameter values. Although both mathematical and heuristic-based solution algorithms use deterministic parameters, the evaluation of the proposed optimal supply chain structure is done with simulation to capture the impacts of those uncertainties. The simulation aims to capture the state of the system in the long term with use of stochastic parameters. Of the biggest advantages of the simulation is its unlimited capacity of introducing stochastic elements and constructing a true representation of the analyzed system. These aspects make simulation a perfect tool to examine the long term consequences of complex supply chain decisions which are usually made using uncertain information.

Vergara et al. [57] developed a heuristic Evolutionary Algorithm (EA) for scheduling the part processes within the suppliers and setting the synchronized delivery cycle time for the supply chain. They compared the results with enumeration of all solutions and showed that the heuristic solution provides optimal or near-optimal solutions faster than enumeration methods as the problem size gets larger. Aytug et al. [4] reviewed the use of a Genetic Algorithm (GA), which is a specific type of EA, to solve production and operations management problems, such as loading, scheduling, facility layout, line balancing, and production planning. The review suggests a rapid increase in heuristic implementations in the last two decades. Several implementation experiments resulted in refined and better heuristic applications that are capable 
of providing good solutions in reasonable computational times. Aytug et al. also stressed that more advanced modeling and implementation techniques and developments in applications of heuristics could be used to solve a wide range of problems. For example, improved solution encoding techniques provide faster heuristic procedures for very complex or large problems since they save computational time by representing solutions in more compact memory.

Truong and Azadivar [56] stated that finding the optimal strategy for a single supply chain would be hard since different components of the supply chain have different, conflicting objectives and the supply chain is a dynamic system that evolves over time. They grouped the supply chain configurations into two categories: (1) structural decisions (location, capacity, and transportation) and (2) coordination decisions (supplier selection, partnerships, inventory ownership, information sharing, demand forecasting, production plans, etc.). They stressed the interactions between all these decisions and the need for considering all at the same time. Their proposed solution algorithm was a hybrid algorithm consisting of a GA (that picks structural variables in particular), MIP (solving for coordination decisions) and simulation (to evaluate the supply chain for random events). Their experiments with illustrative examples showed that this approach performed better than a pure GA application and a random sampling approach in terms of both solution quality and solution time. Nevertheless, further testing with larger instances would be beneficial to observe the quality of the solution procedure, since the authors generated a single-product supply chain with few supplier alternatives and a total of 12 production stages. The model is solved for a single period and single objective (cost minimization) for an existing product. The multi-period, multi-product extension of the model is needed for a more realistic application and multi-objective optimization exists as a challenge for this kind of hybrid solution algorithm. 
Wu and O’Grady [64] developed a Constraint-Based Genetic Algorithm (CBGA) which feeds possible supply chain network design alternatives into the developed Extended Trans-Nets supply chain model. However, complex supply chain model representation of Extended TransNets requires too much computer memory. In addition, the CBGA uses a weighted average of multiple objectives in order to evaluate a solution's fitness which diminishes the benefits of developing a multi-objective model.

Lourenco [46] surveyed application of metaheuristics in various kinds of supply chain models, including product design, inventory planning, and supply chain network design. She suggests that their modularity, easy implementation, easy updating, and adaptation to new situations combined with simulation and decision support systems make metaheuristics an important solution method for solving large scale supply chain network problems.

In parallel to the developments in problem formulations, combinations of several solution techniques (e.g., deterministic optimization techniques, simulation, heuristics, and system dynamics) are more commonly used to solve these large supply chain models. The introduction of stochastic or nonlinear elements and the growing size of the real-world supply chain models (i.e., more variables and parameters due to globalization and outsourcing) requires hybrid solution methods to be utilized to ensure quality solutions with reasonable computational times.

\subsection{SUMMARY}

The provided literature review summarizes how the research interests of different disciplines are focused on the product design and supply chain problems through the past six decades. In addition to the growing interests in these two distinct problems, in the last two decades, it is also 
acknowledged that interactions of these two problems propose important research challenges as the industry needs grow with the globally marketed products and global supply chains.

Different proposed methods try to quantify product design and customer satisfaction relationship. However, these methods often capture the relationship partially due to the highly qualitative nature of the customer behavior. Development of more realistic concepts and methods in establishing the quantitative representation of this interaction is still a significant research challenge.

On the other hand, because of its more quantitative nature, supply chain problems are often modeled in a more structured way. However, although different supply chain models are proposed and even implemented in industry cases, the lack of a consensus on supply chain modeling is still a major drawback. A more significant problem with the supply chain research is that the models tend to be very large for real cases and thus it is very hard to find optimal or even acceptable solutions within adequate time limits. In this case, heuristics and metaheuristics are good candidates as alternative solution techniques when one does not want to compromise from the realistic models. These heuristics are still being developed and improved and finding suitable and fast heuristics for various supply chain problems is still an important research area.

Finally, there has not been a significant progress in Design for Supply Chain research after the mid 1990's until the last couple of years which is now a more challenging task due to the global scale of the product design and supply chain problems. Up to this time, the DFSC models were geared more towards optimizing the supply chain policies and the product differentiation and product family decisions without considering marketing aspects of the product design. Therefore, the modeling of impacts of the product design on customer satisfaction (thus on the product demand) and incorporating the design of the supply chain (not 
only supply chain policies but the actual suppliers and their connections) still poses a significant research problem.

In this research, the product design is looked at both from the marketing and the production perspectives and its interactions with the customer satisfaction and the supply chain performance are investigated. The impacts of product demand and various supply chain performance metrics are captured and translated into financial metrics. In addition, different solution techniques including deterministic optimization and heuristics are investigated and their performances are analyzed. In brief, this research aims to fill in the gap by combined modeling of product design - demand generation, supply chain - demand satisfaction, and product design - supply chain performance interactions as well as investigating efficient solution techniques. 


\subsection{PROBLEM FORMULATION}

In this section, the DFSC problem formulation, which is based on the literature and industry experts' reviews, is presented. Following the detailed problem definition, the preliminary models used in the early steps of the solution method analysis are discussed. Next, the complete mathematical notation and formulation is given. In the subsequent sections, the reduced mathematical models from the complete formulation are provided with a brief asymptotic size analysis of the models.

\subsection{PROBLEM DEFINITION}

This research targets DFSC problems to find optimal product and supply chain design decisions within the product design alternative and supplier selection contexts. DFSC considers constructing the optimal supply chain configuration and product design simultaneously, so that impacts of the supply chain on the selected objectives are optimized during the product life cycle.

The product design phase begins with design requirements and ends with a product description [65]. It can be grouped into four parts: (1) conceptual design phase (usually where a hypothetical design and product function alternatives are created rather than actual detailed drawings are developed), (2) physical design phase (where general features and design 
specifications of the product are determined and drawn as well as some prototyping), (3) detailed design phase (where single components of the product are designed in detail and tested for satisfactory functionality), and (4) final design phase (where the component and final product designs are tested, finalized, and documented). Figure 4 illustrates the details of the design phase within the product life cycle. These design phases are not explicitly distinct and they contain feedforward and feedback loops between these phases since each phase creates inputs for the subsequent phases and undesired results in any phase require adjustments in the previous phases.

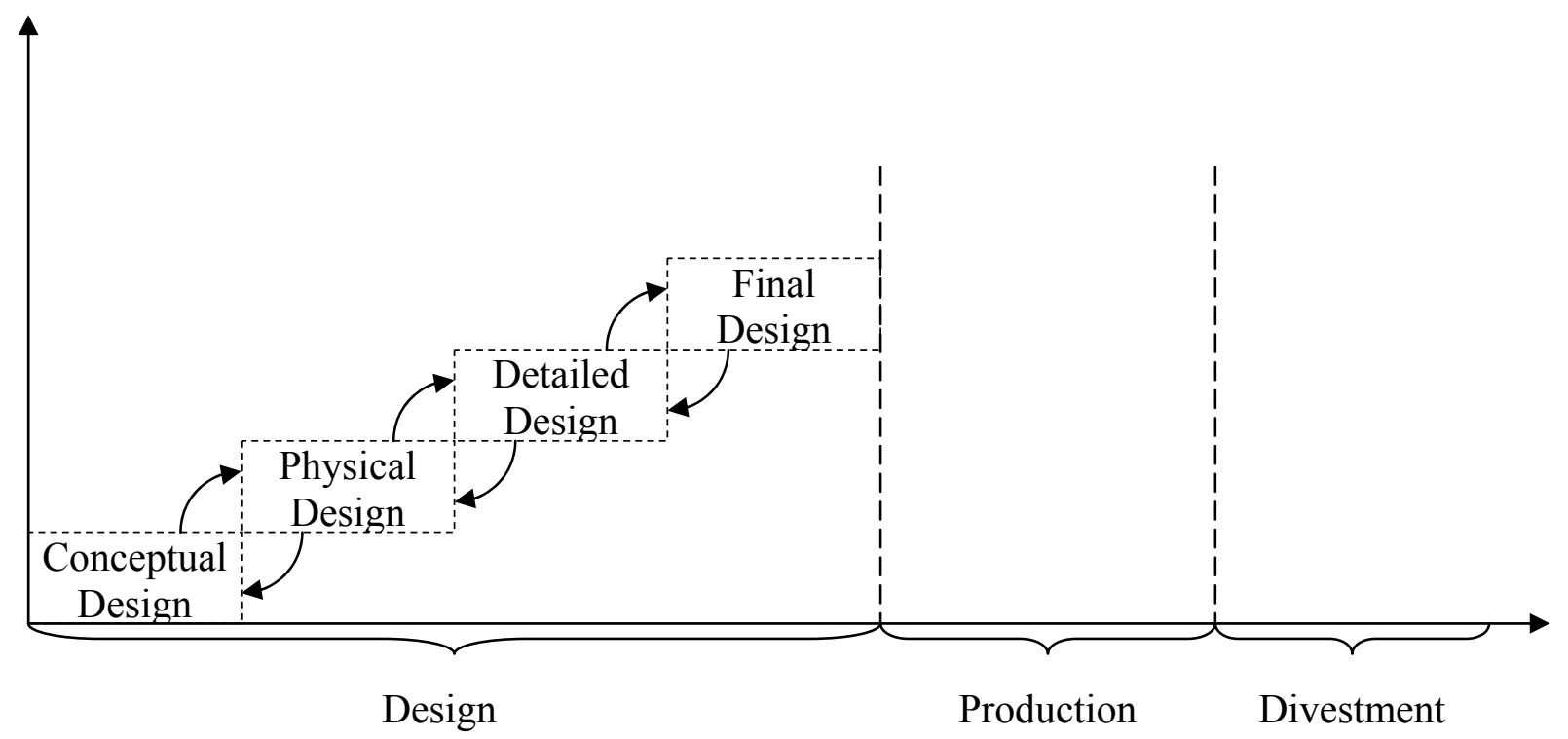

Figure 4: Design phase in the product life cycle

For a new product, the design phase generally uses customer expectations and marketing goals of the company as inputs for the conceptual phase. With these inputs, the main functions of the product are determined. For example, a car manufacturer may decide to launch a new car and the marketing team decides on the targeted customers. The demand for a new product, complaints about the performance of an existing product, or the failure due to malfunctions of an existing 
product can trigger a new product design. At this point, it should be noted that the product design phase is also applicable for redesigning an existing product. This redesign process would not be too different than designing a completely new product other than shorter time requirements especially for the conceptual phase due to existing knowledge. The customer expectations are usually used in the conceptual design phase to define the broad scope of design requirements, such as how powerful, or how technologically advanced the product should be. The pricing requirements are also determined in this phase in general, leading to a Target Costing (TC) procedure to determine an acceptable cost for the final product.

In this research, it is assumed that the product's main function, the general product definition, customer expectations, and the customers' assessments about functions of the components are known. Therefore, the market research efforts to understand what customers require and how they value each available feature related to new or redesigned products are not modeled. Instead, this information is assumed to be already known and used as an input for the DFSC model.

With these assumptions, the problem is defined to be selecting the "optimal" product design. This optimal design is aimed at achieving maximum profit by generating desired demand through satisfying customer needs and by utilizing the supply chain optimally. Here, an optimal supply chain is defined to have lower cost with acceptable lead time and to provide high quality products. Obviously, the demand generation and satisfaction are not only functions of a product design, but also significant outcomes of the supply chain performance thus leading to the DFSC concept. Figure 5 illustrates the proposed DFSC procedure. 


\begin{tabular}{|c|c|c|c|c|}
\hline \begin{tabular}{|c|} 
Define main \\
product (aim of the \\
product)
\end{tabular} & \begin{tabular}{|} 
Use VE to determine the \\
customer requirements / \\
possible pricing \\
information
\end{tabular} & $\begin{array}{c}\text { Use TC to determine } \\
\text { a product cost }\end{array}$ & $\begin{array}{l}\text { Determine product } \\
\text { components }\end{array}$ & $\begin{array}{c}\text { Use VE to set } \\
\text { component } \\
\text { specifications / TC for } \\
\text { component costs }\end{array}$ \\
\hline
\end{tabular}

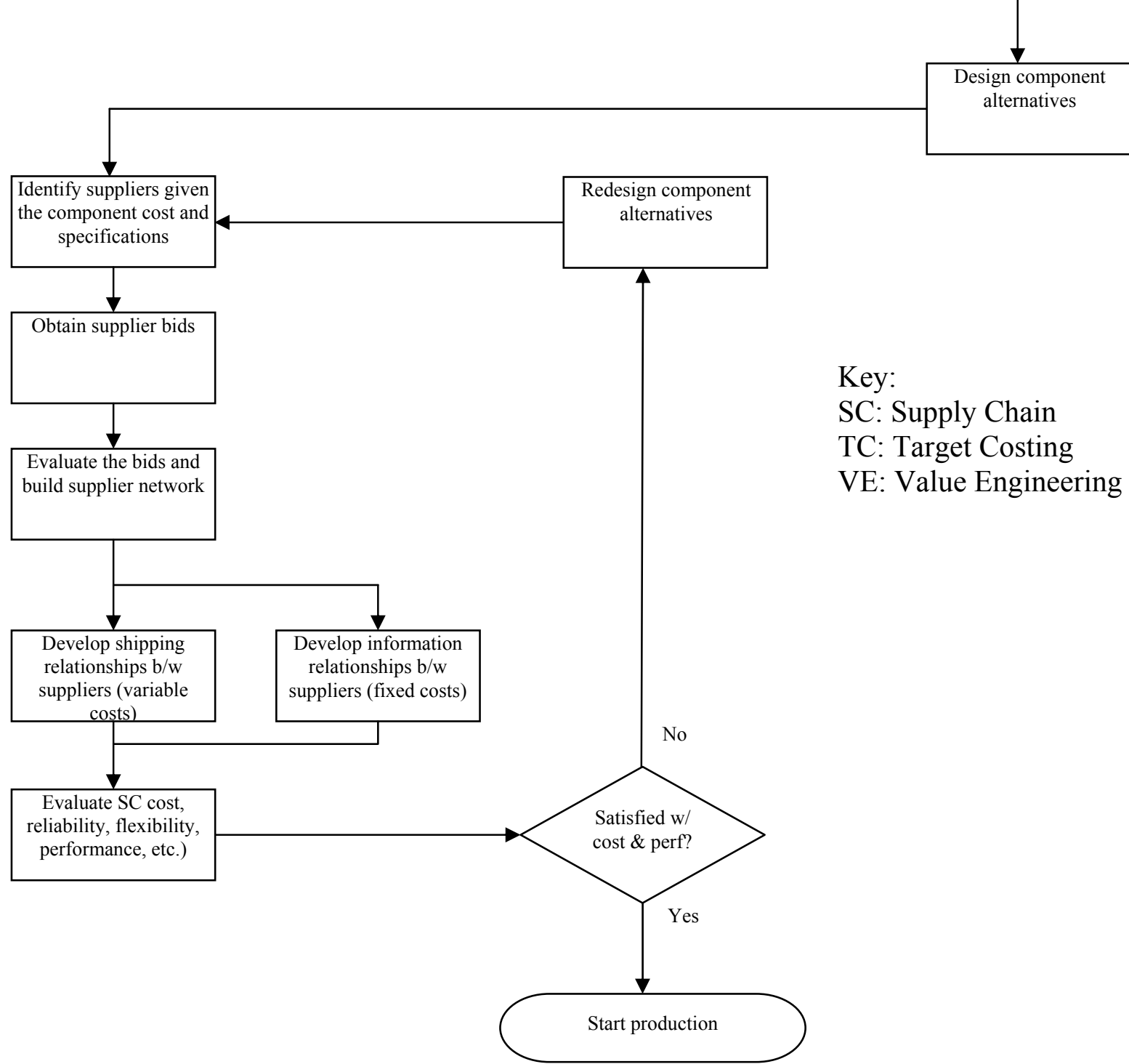

Figure 5: The proposed DFSC Procedure 
The proposed DFSC procedure starts with the definition of the main product. Based on this decision, Value Engineering, market surveying, and similar tools are utilized to understand how much value each product feature would add to the product from the customer point of view. This information is later used in deciding among product design alternatives. At the same time, product definition, value assessment, and market survey results are combined to develop a possible pricing range for the final product. Given that price range and company's profit goals, a target product cost is established. In the next step, by employing customer preferences and proposed features of the product, different product components are determined. Subsequently, customer value assessment and target cost information is distributed among these product components. For example, initial customer surveys for a car design may suggest a certain value for the safety of the car. However, this value needs to be distributed among several components related to safety such as braking systems, traction controls, airbags, and seat belts either by industry experts or again by customer survey methods. In the next step, different design alternatives for each component are designed. These design alternatives might be actual different geometric component designs or may consist of different design tolerances, different materials, and regional customization options. In parallel to the design alternatives development, potential suppliers are searched. For each component design, supplier's capabilities, capacity, cost, lead time, and quality information is collected. Finally, the proposed DFSC approach optimizes the product and supply chain designs concurrently by balancing demand generated by the product design and demand satisfied through the associated supply chain. The details of different modeling assumptions and concepts captured by the model formulation are explained below. 


\section{Objective Function}

Since the developed DFSC model aims to balance the product and supply chain designs, different objectives can be sought. In the literature, the supply chain optimization models mostly aim to minimize total cost of the supply chain [7]. However, in reality, a company's final objective from a product would always be to generate maximum profit by creating demand for this product through marketing and then by satisfying this demand through manufacturing and sales. Fandel [22] suggests that "as a component of strategic planning, strategic supply chain management is focused towards the goals and tasks of company policy. The main goal of strategic supply chain management is to achieve profit maximization." In their simultaneous product and supply chain design study, Lamothe et al. [37] stressed that when market shares (and demand) depend on the product configuration (options chosen), the eventual objective needs to be profit maximization. Since the DFSC models developed in this research consider both marketing and manufacturing decisions towards creating and satisfying the demand, the final objective is selected to be overall profit maximization throughout the product's life cycle.

\section{Marketing Mix Assumptions}

In terms of marketing mix decisions, price and product (as product design) are modeled since they are the most relevant decision variables in profit maximization. For simplification purposes and in support of the main objective of understanding product and supply chain design interactions, impacts of product placing and promotion are excluded.

\section{Product Design's Impacts on the Demand and Supply Chain}

In this research, the product design plays three important roles. The first one is that the value of each component alternative design is added to determine the final value of the product. The total demand for the product is positively correlated to the value of the product design thus a 
better product design would increase the demand or vice versa. In order to quantify the customers' needs and value assessments, each product component is given a value between 0 and 1. These component values add up to 1 , representing a percentage of the corresponding component's value satisfying the customers' needs. For example, a computer basically consists of a motherboard-CPU, a hard disk, a monitor, memory, and other peripherals. If the monitor is valued at $20 \%$ importance by customers then it would be valued as 0.2 in the model. The remaining components' values would sum up to $80 \%$ (or 0.8 in the model). Within each component, several design alternatives are considered. Each of these alternatives has different values with a maximum of the value of the component itself. For example, an LCD monitor may be valued at $20 \%(0.2)$ due to its better view quality and smaller size where a CRT monitor may be valued at $10 \%(0.1)$ due to its old technology and larger space requirements. The important point here is that a design alternative's value should never exceed the maximum value of the component itself ( $20 \%$ for a monitor in the example). In this model, all component designs are assumed to be compatible with each other allowing the model to select the product designs without any restrictions. However, for a given time period only one design alternative for each component can be selected since concurrent selection of different design alternatives would mean different products at the end and would require a multi-product model. At the same time, it is assumed that there is no correlation between the value of a component design and the associated supplier manufacturing and transportation costs and lead times. This implies that a higher valued component design may cost less than a less valued component design to manufacture or transport. For example, a (high valued) LCD monitor takes less space than a regular (low valued) CRT monitor resulting in lower transportation costs. 
The second role of the product design is that it limits supplier alternatives for the supply chain network since each component design alternative may not be manufactured by each supplier. Within the same computer design example, an LCD monitor design would result in a different suppliers set than a CRT monitor since an LCD is an advanced technology and still may not be manufactured by some suppliers.

\section{Obsolescence}

Finally the third role of the product design is that, by utilizing different component alternative values over the time periods, it captures the impacts of the obsolescence which is an important product design performance metric in today's fast paced business environment. For example, a high valued component design for the initial time periods might have a lower design value for the later time periods. Since this value is directly linked with the product's demand generation potential, a lower value represents lower demand due to obsolescence of the particular

component design. It should be noted that in this model obsolescence is also captured by diminishing supplier capacities over the time periods for certain component design alternatives. For example, in a year, a supplier may have half the capacity it has today for a particular component alternative due to unavailability of raw materials, lower profitability for the supplier, and market competition. Therefore, the model captures obsolescence both by accounting for reduction in demand and reduction in supply as the component design alternative becomes obsolete.

\section{The Demand Function and Demand Assumptions}

As it is stated that price and product (as product design) factors of the marketing mix are used in the model, the demand function consists of their interactions. Various functions are used in the marketing, economy, and engineering literatures to represent price - demand relationship 
including linear, polynomial, exponential, logistic, etc. In this research, a quadratic function is used to relate the pricing decisions to the demand since it captures the changing price elasticity of the demand for different pricing ranges without complicating the mathematical model.

The impact of the product design on the demand is assumed to be linearly correlated (positive) with the product design value. According to this approach, the maximum design value,

1 , generates the maximum demand where any lower product design value reduces the demand in a linear fashion. For example, a product design value of 0.5 would generate half of the maximum demand.

A third element of the demand function is the time period itself, where the demand occurs. This element helps capture the impacts of different product life cycle phases on the demand such as having a lower demand during the introduction period and a higher demand during the product maturity phase given that same price levels and product design features are provided in each period.

The interaction of these three elements (price, product design, and time period) is assumed to impact the demand in a combined way. Therefore, each element's impact is multiplied toward the final demand calculation, leading to a non-linear demand function due to a quadratic price function and multiplication of price and design value variables. A demand function linearization schema is provided in Appendix section A.2.

\section{Supply Chain Performance Metrics}

For the supply chain planning models, Weber [62] suggested that manufacturing cost, lead time, product quality, supplier capacity, and supplier location are the criteria most often used as primary decision factors. Together with the industry experts' opinions (see Section 5.2), primary supply chain criteria are selected to be manufacturing, inventory, and transportation 
costs, lead time, and supplier capacity. Quality of each supplier is assumed to be at least at a certain level that they are considered in the planning. Therefore, an assumption of the model is that all the available suppliers meet the quality specifications of a particular component or they are not considered for being candidates and have a zero capacity for that particular component. Supplier locations are also not explicitly modeled for simplification; however the impacts of the location on the transportation costs and lead times are captured by supplier specific information. Other than these two main aspects, the supplier location impacts are mostly qualitative in nature such as risks and opportunities. Although these measures can be added into the model later, they do not have a direct impact on the product and supply chain design interaction and thus are not included.

\section{Economies of Scale Structure and Assumptions}

The supplier manufacturing costs are included as unit costs incurred per item manufactured. These costs change for selected component design alternatives, suppliers, and time periods. In addition, supplier capacities may differ for each time period, for the component design alternative, and for different suppliers. In order to capture the impacts of the economies of scale, which suggests lower unit manufacturing costs for larger order sizes, the supplier capacities and manufacturing costs are divided into two levels. Up to the first supplier capacity level (which would represent a partial use of the total capacity), a relatively higher unit manufacturing cost is incurred per unit. Only if this level 1 capacity is fully utilized, then additional units that fall into level 2 capacity would be charged a lower unit manufacturing cost. This model representation captures the concept of reducing per unit manufacturing costs with higher capacity utilization. It should be noted that the economies of scale factor in this model is 
only related to unit manufacturing costs and has no impact on transportation and supply chain network costs and lead times.

\section{Transportation Structure and Assumptions}

In this model, different transportation costs are incurred for different directions of flow between suppliers, for different component alternative designs, and for different time periods. This structure helps to capture different import / export taxes applied by countries. For example, if a country (or region) only applies import taxes and omits export taxes, then it would cost less to receive from that country than to send to that country. In addition, this structure allows the same components to be manufactured by different suppliers and transported to various highertiered suppliers independently. Briefly, components are not required to be transported from / to a single supplier and any number of suppliers can manufacture the same component and transport to a single supplier or different suppliers.

\section{Lead Time Concept and Assumptions}

As one of the important supply chain performance metrics, lead times are considered as the summation of a components' and its predecessors' production times. Lead times are assumed to include both production and transportation times. Each lead time is defined for a component design alternative from a supplier for a given time period. However, lead times are assumed to be given for predetermined batch sizes and they only depend on the supplier capacity utilization. In lead time calculations, specific times of the suppliers are not considered in calculating the final lead time and instead, the longest time of the all suppliers (relative to the supplier capacity utilization) is assumed as a component's lead time. This means production is assumed to continue only when all the required components are delivered. Partial deliveries from different suppliers with shorter lead times are not accounted toward a component's final lead time. 


\section{Inventory Calculations and Assumptions}

The lead time information is not directly modeled in the objective function but rather its impacts on the inventory costs are used. Total inventory cost for a time period is approximated by assuming that final product inventory is held by the main manufacturer at a level that ensures a certain level of demand is satisfied during the lead time period. This demand satisfaction level is given by the end user and corresponding safety stock levels depend on this satisfaction level. Therefore, longer lead times results in higher safety stock levels driving inventory costs higher. By this representation, the model captures a lead time minimization objective via an inventory cost minimization perspective. In order to capture the variability of the demand and lead time in a deterministic model, it is assumed that demand and lead time have constant coefficients of variations ( $\rho_{l}$ and $\rho_{2}$, respectively) thus higher lead time (and demand) values result in higher variations. Moreover, for simplification purposes and to keep a linear model, demand variability is removed from the inventory calculations $\left(\rho_{l}=0\right)$.

\section{Time Periods in the Model}

As this model aims to optimize product and supply chain design at a strategic (long term) level, time periods are considered to be product life cycle phases. These phases often last for months or even years. Therefore, this strategic level model does not deal with production scheduling and short term inventory planning problems.

\section{Supply Chain Network Costs}

Another important supply chain cost driver is the initial investment costs of the supply chain network. These costs include supplier certification, training, computer and communication network infrastructure (including computer software investments), and other initial, one-time fixed costs. Although the qualitative preferences over certain suppliers (such as supplier 
preference weights) are not explicitly modeled, the model captures some of these cost-based preferences by accounting for different supply chain network costs. For example, a strategic supplier, which the company has had business with, would have significantly lower supply chain network costs since minimal certification or communication investments would be required for a new product. These costs are modeled in a way that when there is a direct relationship between any two suppliers, the associated supply chain network costs are incurred. The direct relationship between two suppliers is defined to exist only if a sub-component is transported from one of the suppliers to the other one. These direct relationships are represented in a one-way fashion, therefore they illustrate that only the sub-component supplier is linked to the upper tier and the corresponding costs are incurred. For an upper-tier supplier to be linked to the lower-tier supplier; it must supply another sub-component to this supplier and this would remove the hierarchy between them, making them both at the same tier. It should be noted that although these supply chain network costs are one-time fixed costs, they are not modeled as facility opening-closing costs which is widely used in the literature. The open-close decision structure limits the model to incur these costs once if a supplier is used regardless of its relationships' complexity within the supply chain. The benefit of this modeling structure is that it allows different supply chain network costs to be incurred helping to capture cases like excluding certification costs for an already certified supplier. It also helps model different costs for each supplier if it has a complex relationship with many of the suppliers. 


\subsection{PRELIMINARY MODELS}

In order to test proposed deterministic optimization and heuristic solution techniques, two preliminary mathematical models are developed. Total cost and lead time are the two primary performance measures that are used in this preliminary analysis. Although other secondary performance measures are mentioned in the literature, only total cost and lead time are used in the preliminary problem formulation stage since this stage aims to evaluate performance of different solution techniques rather than developing the complete DFSC model. The product design, pricing decisions, and their impacts on the demand generation are also excluded in the preliminary modeling stage.

In the preliminary analysis, the total cost is considered to have two parts: (1) total production costs (e.g., setup, material, and labor costs) and (2) total supply chain network costs. Total production costs are assumed to be varying by production quantity and they are given per unit. For simplicity reasons transportation, distribution, inventory holding, and similar production costs are excluded in this stage. Total supply chain network costs are assumed to be fixed costs that are charged independent of the number of units produced by the main manufacturer or its suppliers. It is assumed that there would be a network (relation) between any supplier pairs (or between the supplier and the main manufacturer) for any directly related components that are manufactured by them. Direct relation between components is defined to occur when one of the components directly goes into the assembly of the other component in the Bill of Material (BOM). For example, if a metal component is directly assembled into a car body without going through any other process (e.g., painting, press, or welding), then there exists a direct relation between the metal component and the car body. Therefore, a network cost is incurred between the manufacturers of this metal segment and car body. It should be noted that 
any supplier who manufactures a component that is used in several other components has network relations with all these manufacturers. However, if a supplier supplies more than one component for another supplier or the manufacturer, the network costs are incurred only once, since these costs are assumed to include initial inspection, training, communication network implementation, and similar one time initial costs. The primary difference of this preliminary supply chain network representation from the method described in the problem definition (section 3.1) is that suppliers are assumed to have a network connection when they manufacture any two directly related components regardless of whether these components are actually transported between them for manufacturing.

With respect to the lead time minimization model, the lead times are assumed to be fixed and independent of the supplier's capacity utilization in the preliminary analysis. Since the transportation aspect is also excluded in the preliminary modeling stage, the lead time values do not include transportation times but only consists of production times.

\section{Cost minimization model}

In this model, the cost is minimized subject to demand satisfaction, economies of scale, capacity limitations, and supply chain network generation constraints. The parameters and variables are as follows:

\section{Parameters:}

Sets $i \in I$ : Sets of components from component 1 (final product) to component $P$

Sets $j \in \mathcal{J}$ : Sets of suppliers from 1 (the main manufacturer) to $S$

$P$ : Total number of components used in the product $(|\mathcal{I}|=P)$

$S:$ Total number of available suppliers $(|\mathcal{J}|=S)$

$c_{i j}{ }^{1}, c_{i j}{ }^{2}$ : Unit manufacturing costs of component $i$ at supplier $j$ for production levels 1 and 2 
Capacity $_{i j}{ }^{1}$, Capacity ${ }_{i j}{ }^{2}$ : Total production capacity of supplier $j$ for component $i$ for production levels 1 and 2.

Demand $_{i}$ : Total demand for component $i$

Netwco jl: Fixed supply chain network costs between suppliers $j$ and $l$

Relation $_{i k}$ : Number of components $k$ required to manufacture component $i$

\section{Variables:}

$x_{i j}{ }^{1}, x_{i j}{ }^{2}$ : Total production amount of component $i$ at supplier $j$ for production levels 1 and 2

$z_{i j}: 1$, if component $i$ is manufactured by supplier $j ; 0$, otherwise

$y_{j l}: 1$, if suppliers $j$ and $l$ have a direct relationship; 0 . otherwise

$a_{i j}: 1$, if supplier $j$ does not fulfill its level 1 capacity with component $i ; 0$, otherwise

$\min \sum_{i=1}^{P} \sum_{j=1}^{S}\left(c_{i j}^{1} x_{i j}^{1}+c_{i j}^{2} x_{i j}^{2}\right)+\sum_{j=1}^{S} \sum_{l=1}^{S} y_{j l} N^{N e t w c o} o_{j l}$

s.t.

$\sum_{j=1}^{S}\left(x_{i j}^{1}+x_{i j}^{2}\right) \geq$ Demand $_{i} \quad \forall i$

Demand satisfaction

$x_{i j}^{1} \leq$ Capacity $_{i j}^{1} z_{i j} \quad \forall i, j$

$x_{i j}^{1} \geq$ Capacity $_{i j}^{1} a_{i j} \quad \forall i, j$

$x_{i j}^{2} \leq$ Capacity $_{i j}^{2} a_{i j} \quad \forall i, j$

$z_{i j}+z_{k l}-1 \leq y_{j l} \quad \forall i, j, k, l \mid$ Relation $_{i k}>0,(j \neq l)$ constraint

Capacity and economies

Supply chain generation

$x_{i j}^{1}, x_{i j}^{2} \geq 0, z_{i j}, y_{j l}, a_{i j} \in\{0,1\}$

Equation (2.1) is the objective function that consists of total production and supply chain network costs. The first part of the equation is the total production costs that is the summation of level 1 and level 2 production costs of components at each supplier. The second part of the equation is the summation of total network costs incurred over all possible supplier relation pairs. The first constraint (equation 2.2) ensures that the total production of each component 
satisfies the demand of this component. Equations (2.3), (2.4), and (2.5) serve both as capacity and economies of scale constraints. While equations (2.3) and (2.5) limit the production of each component with the related supplier's capacity limitations for different production levels, equation (2.4) determines the value of the $a_{i j}$ variable by checking whether level 1 production capacity is fulfilled. Unless this level 1 capacity is fully utilized, the $a_{i j}$ variable prohibits use of any level 2 capacity by interacting in equation (2.5). Equation (2.3) also controls the $z_{i j}$ variable by forcing it to be 1 when a production quantity is assigned to supplier $j$ for component $i$. Equation (2.6) is used to capture the network relationships within the supply chain by ensuring that the $y_{j l}$ variable gets a value 1 when suppliers $j$ and $l$ manufacture directly related components so that appropriate network relation costs are incurred in the objective function. Equation (2.7) defines the structure of the variables.

\section{Lead time minimization model}

In this model, the total time required to manufacture the final product (lead time) is minimized subject to demand and capacity constraints. The same variables and parameters as used in the cost minimization model apply, with the addition of the following:

\section{Parameter:}

$p t_{i j}$ : Production time of component $i$ at supplier $j$

\section{Variables:}

$L T_{i}$ : Total lead time for component $i$

ptime $_{i}$ : Maximum production time for component $i$ 
$\min L T_{1}$

s.t.

$\sum_{j=1}^{S}\left(x_{i j}^{1}+x_{i j}^{2}\right) \geq$ Demand $_{i} \quad \forall i$

Demand satisfaction constraint

$x_{i j}^{1} \leq$ Capacity $_{i j}^{1} z_{i j} \quad \forall i, j$

$x_{i j}^{1} \geq$ Capacity $_{i j}^{1} a_{i j} \quad \forall i, j$

$x_{i j}^{2} \leq$ Capacity $_{i j}^{2} a_{i j} \quad \forall i, j$

ptime $_{i} \geq$ pt $_{i j} z_{i j} \quad \forall i, j \mid$ Capacity $_{i j}^{1}>0$

$L T_{i} \geq L T_{k}+$ ptime $_{i} \quad \forall i, k \mid$ Relation $_{i k}>0$

Capacity and economies

of scale constraints

Lead time calculation

$L T_{i} \geq$ ptime $_{i}$ constraints

$x_{i j}^{1}, x_{i j}^{2}$, ptime $_{i}, L T_{i} \geq 0 ; z_{i j}, a_{i j} \in\{0,1\}$

The objective function (equation 3.1) minimizes the lead time of component $1\left(L T_{1}\right)$, which is defined as the main product. Equation (3.2) ensures the demand for each component is satisfied where equations (3.3), (3.4), and (3.5) limit the production levels according to the capacity limits and dictate proper use of the economies of scale concept as described with the cost minimization model. Equation (3.6) ensures that production time of component $i$ is selected as the maximum of all production times of component $i$ among all suppliers that manufacture this component. This is necessary to capture the impact of selecting the supplier with longer lead times, since in general manufacturing a batch of product starts after its components arrives. Thus, if one supplier takes longer to deliver a component than another supplier, the production waits for the supplier with the longer lead time. The next constraint (3.7) defines the lead time of a component $\left(L T_{i}\right)$ as the summation of this component's production time and the maximum of related component's lead time values (since basically lead time values are based on production times). Equation (3.8) ensures that lead time of a component is at least equal to its production time. It can be suggested that equation (3.7) already includes equation (3.8), but to reduce the number of constraints in the model, equation (3.8) is defined only for those component pairs that have a direct relationship. In 
this case, equation (3.8) is required to capture the lead and production time relation for the components that do not have any components used in them. Finally, equation (3.9) defines the structure of the variables.

\section{Reduced cost minimization model}

In addition to these preliminary models, another model is used to optimally assign the production orders within the heuristic optimization procedure. This model is an MIP model that minimizes total production costs without considering supply chain network costs. Therefore, this model is actually a subset of the equations given in the cost minimization model. This model is given below with the variable and parameter definitions as defined previously.

$\min \sum_{i=1}^{P} \sum_{j=1}^{S}\left(c_{i j}^{1} x_{i j}^{1}+c_{i j}^{2} x_{i j}^{2}\right)$

s.t.

$\sum_{j=1}^{S}\left(x_{i j}^{1}+x_{i j}^{2}\right) \geq$ Demand $_{i} \quad \forall i \quad$ Demand satisfaction constraint

$x_{i j}^{1} \leq$ Capacity $_{i j}^{1} z_{i j}$

$\forall i, j$

$x_{i j}^{1} \geq$ Capacity $_{i j}^{1} a_{i j}$

$\forall i, j\}$ Capacity and economies of scale constraints

$x_{i j}^{2} \leq$ Capacity $_{i j}^{2} a_{i j}$ $\forall i, j \quad\}$

$x_{i j}^{1}, x_{i j}^{2} \geq 0, z_{i j}, a_{i j} \in\{0,1\}$

The solution techniques test results based on these preliminary models are given in sections 4.1.2 and 4.2.2.

\subsection{COMPLETE MODEL FORMULATION}

The complete DFSC model as presented here is based on the conditions and assumptions explained in problem definition (section 3.1). This model aims to maximize the total profit over 
the product's life cycle by selecting component design alternatives, prices, and supplier production and transportation quantities. The product demand, supply chain network structure, and inventory levels are also calculated. Obsolescence and economies of scale concepts are captured via different constraints. For the completeness of the model, all used parameters and variables (including ones that were used in preliminary models) are listed below:

\section{Parameters:}

- Sets $i \in I$ : Sets of components from component 1 (final product) to component $P$

- Sets $j \in J$ : Sets of suppliers from 1 (the main manufacturer) to $S$

- Set $\alpha_{i} \in \mathcal{A}_{i}$ : Set of design alternatives of component $i$

- Set $t \in \mathcal{T}$ : Set of time periods

- Set $n \in \mathcal{N}$ : Set of binary factorization elements of lead time - demand multiplication

- $\quad P$ : Total number of components used in the product $(|\mathcal{I}|=P)$

- $\quad S$ : Total number of available suppliers $(\mid \mathcal{J}=S)$

- $A_{i}$ : Number of design alternatives for component $i\left(\left|\mathcal{A}_{i}\right|=A_{i}\right)$

- $\quad T$ : Number of time periods (each representing a product life cycle phase) $(|\mathcal{T}|=T)$

- $\quad N$ : Number of lead time - demand binary variables $\left(\gamma\right.$ and $\delta$ ) that cover all possible $L T_{1 t} \times$ Demand $_{1 t}$ values in a binary representation $(|\mathcal{N}|=N)$

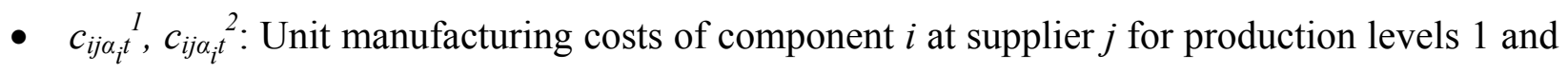
2 for component design $\alpha_{i}$ at time period $t$

- Capacity $_{i j j_{i}}{ }^{l}$, Capacity $_{i j \alpha_{i}}{ }^{2}$ : Total production capacity of supplier $j$ for component $i$ for production levels 1 and 2 for component design $\alpha_{i}$ at time period $t$ 
- Netwco jl: Fixed supply chain network costs between suppliers $j$ and $l$

- Relation $i k$ : Number of components $k$ required to manufacture component $i$

- $p t_{i j \alpha_{i}}$ : Production time of design $\alpha_{i}$ of component $i$ at supplier $j$ in time period $t$

- $v a l_{i_{i} t}$ : Value of design $\alpha_{i}$ of component $i$ for the demand in time period $t$ (\% of total contribution)

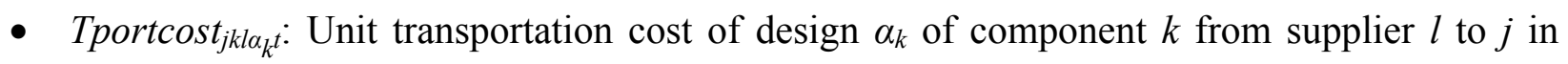
period $t$

- $\omega_{1 t}, \omega_{2 t}, \omega_{3 t}$ : Allowed values that price can take in time period $t$

- $h_{t}$ : Unit inventory holding cost of the final product in time period $t$

- $\beta_{1}, \beta_{2}$ : Demand function coefficients

- timemultiplier $_{t}$ : A parameter value in order to adjust demand value according to the time period $t$ (based on what life cycle phase $t$ is)

- periodlength : $_{\text {: }}$ ength of the time period $t$ (in the same units with lead time)

- $z_{\text {ssratio: }}$ z-value from the normal distribution corresponding to the given safety stock ratio (ssratio)

- $\rho_{l}, \rho_{2}$ : Constant coefficients of variation for demand over lead time and lead time, respectively

- Mcapia $\alpha_{i}$ : Total available capacity for design $\alpha_{i}$ of component $i$ in time period $t$ over all suppliers $\left(\sum_{j=1}^{P}\right.$ Capacity $_{i j \alpha_{i} t}^{1}+$ Capacity $\left._{i j \alpha_{i} t}^{2}\right)$

- Mdem: Maximum potential demand over all periods (calculated by using maximum of timemultiplier $_{t}$ and lowest price $_{t}$ with $v_{t}=1$ ) 


\section{Variables:}

- $\quad x_{i j \alpha_{i}{ }^{t}}{ }^{t}, x_{i j j_{i}{ }^{2}}{ }^{2}$ : Total production amount of component $i$ at supplier $j$ for production levels 1 and 2 for component design $\alpha_{i}$ at time period $t$

- price $_{t}$ : Price of the final product in time period $t$

- $y_{j l}: 1$, if suppliers $j$ and $l$ have a direct relationship; 0 , otherwise

- $a_{i j \alpha_{i} i}: 1$, if supplier $j$ fulfills its level 1 capacity with design $\alpha_{i}$ of component $i$ in time period $t$; 0 , otherwise

- $u_{j k l \alpha_{k}}$ : Total amount of design $\alpha_{k}$ of component $k$ manufactured at supplier $l$ and transported to supplier $j$ in time period $t$

- $\pi_{i \alpha_{i} t}: 1$, if design $\alpha_{i}$ of component $i$ is selected for time period $t ; 0$, otherwise

- $v_{t}$ : Total value of the final product design for time period $t$ (between 0 and 1 , calculated by a constraint in the model)

- Demand $_{i t}$ : Total demand for component $i$ at time period $t$

- $\phi_{1 t}: 1$, if price values are increased to $\omega_{2 t} ; 0$, otherwise

- $\phi_{2 i}: 1$, if price values are increased to $\omega_{3 i} ; 0$, otherwise

- $\lambda_{1 \mathrm{t}}, \lambda_{2 \mathrm{t}}$ : Variables that reflects pricing decision onto demand generation via $\phi_{1 t}, \phi_{2 t}$, and $v$ in time period $t$

- $\tau_{t}^{+}: 1$, if demand $>$ total production; 0 , otherwise

- $\tau_{t}^{-}: 1$, if total production $>$ demand; 0 , otherwise

- $k_{t}^{+}$: Equal to demand, if demand $>$total production; 0, otherwise

- $k_{t}$ : Equal to total production, if total production > demand; 0 , otherwise 
- $\psi_{1,}, \psi_{2 t}, \psi_{3 t}, \psi_{4 t}$ : Control variables that link pricing decisions and demand or total production values for revenue calculation in time period $t$

- $L T_{i t}$ : Total lead time for component $i$ in time period $t$

- LTint $t: L T_{1 t}$ value rounded up to the nearest integer

- ptime $_{i t}$ : Maximum production time for component $i$ in time period $t$

- $\gamma_{n t}: 1$, if $\mathrm{n}^{\text {th }}$ binary factor for $L T_{l t}$ is selected for time period $t ; 0$, otherwise

- $\delta_{\mathrm{nt}}$ : A variable to reflect lead time-demand multiplication via binary factorization in time period $t$

The complete DFSC model is described below:

\section{Objective Function}

The objective of this model is to maximize the total profit throughout the product's life cycle. Two components of the total profit are total revenue and total cost and total profit is defined as

$$
\text { Total Profit }=\text { Total Revenue }- \text { Total Cost }
$$

Since the model is not restricted to satisfy all the demand, the Total Revenue would be

$$
\text { Total Revenue }=\text { Price } x \text { Satisfied Demand }
$$

where,

$$
\text { Satisfied Demand }=\min (\text { Demand, Total Production })
$$

Since both demand and production amounts are variables in the model, for the Total Revenue calculation, a linearization schema is developed. The details of this linearization are given in Appendix section A.1. According to this schema, Total Revenue over the product's life cycle is

$$
\text { Total Revenue }=\sum_{t=1}^{T} \omega_{1 t}\left(k_{t}^{+}+k_{t}^{-}\right)+\left(\omega_{2 t}-\omega_{1 t}\right)\left(\psi_{1 t}+\psi_{3 t}\right)+\left(\omega_{3 t}-\omega_{1 t}\right)\left(\psi_{2 t}+\psi_{4 t}\right)
$$


On the other hand, Total Cost consists of all supply chain related costs, namely production, supply chain network, transportation, and inventory costs. The Total Production Cost is the summation of all manufacturing costs incurred by all the suppliers for all selected component design alternatives over the planning horizon. Therefore, it can be expressed by

$$
\text { Total Production Cost }=\sum_{t=1}^{T} \sum_{i=1}^{P} \sum_{j=1}^{S} \sum_{\alpha_{i}=1}^{A_{i}}\left(c_{i j \alpha_{i}}^{1} x_{i j \alpha_{i} t}^{1}+c_{i j \alpha_{i} t}^{2} x_{i j \alpha_{i} t}^{2}\right)
$$

The supply chain network costs are incurred whenever there is a direct relationship between two suppliers. Hence, Total Supply Chain Network Cost is the summation of these costs over all supplier pairs and given as

$$
\text { Total Supply Chain Newtork Cost }=\sum_{j=1}^{S} \sum_{l=1}^{S} y_{j l} \text { Netwco }_{j l}
$$

The transportation costs are incurred per unit transported, so the Total Transportation Cost is the summation of costs incurred per item for each component design alternative between all supplier pairs over the planning horizon as represented below.

$$
\text { Total Transportation Cost }=\sum_{t=1}^{T} \sum_{j=1}^{S} \sum_{k=1}^{P} \sum_{l=1}^{S} \sum_{\alpha_{k}}^{A_{k}} u_{j k l \alpha_{k} t} \text { Tportcost }_{j k l \alpha_{k} t}
$$

Finally, Total Inventory Cost constitutes the last group of cost drivers. Due to the non-linear nature of the safety stock calculations, the Total Inventory Cost function is linearized. The details of the linearization schema are given in Appendix section A.3. The Total Inventory Cost is the summation of Inventory Costs over all time periods and given below.

$$
\text { Total Inventory Cost }=\sum_{t=1}^{T} \frac{1}{2} h_{t}\left(1+z_{\text {ssratio }} \rho_{2}\right) \sum_{n=0}^{N} 2^{n} \delta_{n t}
$$

Given these components of the objective function, the complete DFSC model is presented below. 


$$
\begin{aligned}
\max & \left(\sum_{t=1}^{T} \omega_{1 t}\left(k_{t}^{+}+k_{t}^{-}\right)+\left(\omega_{2 t}-\omega_{1 t}\right)\left(\psi_{1 t}+\psi_{3 t}\right)+\left(\omega_{3 t}-\omega_{1 t}\right)\left(\psi_{2 t}+\psi_{4 t}\right)-\right. \\
& \sum_{t=1}^{T} \sum_{i=1}^{P} \sum_{j=1}^{S} \sum_{\alpha_{i}=1}^{A_{i}}\left(c_{i j \alpha_{i} t}^{1} x_{i j \alpha_{i} t}^{1}+c_{i j \alpha_{i} t}^{2} x_{i j \alpha_{i} t}^{2}\right)-\sum_{j=1}^{S} \sum_{l=1}^{S} y_{j l} \text { Netwco }_{j l}- \\
& \left.\sum_{t=1}^{T} \sum_{j=1}^{S} \sum_{k=1}^{P} \sum_{l=1}^{S} \sum_{\alpha_{k}=1}^{A_{k}} u_{j k l \alpha_{k} t} \text { Tportcost }_{j k l \alpha_{k} t}-\sum_{t=1}^{T} \frac{1}{2} h_{t}\left(1+z_{\text {ssratio }} \rho_{2}\right) \sum_{n=0}^{N} 2^{n} \delta_{n t}\right)
\end{aligned}
$$

s.t.

Demand $_{1 t}=\left(\left(\beta_{1} \omega_{1 t}^{2}+\beta_{2}\right) v_{t}+\beta_{1} \lambda_{1 t}\left(\omega_{2 t}^{2}-\omega_{1 t}^{2}\right)+\beta_{1} \lambda_{2 t}\left(\omega_{3 t}^{2}-\omega_{1 t}^{2}\right)\right) \times$ timemultiplier $_{t} \quad \forall t$

Demand $_{k t}=\sum_{\mathrm{i}=1}^{\mathrm{k}-1}$ Demand $_{i t}$ Relation $_{i k} \quad \forall k(k>1), t$

Demand satisfaction
constraint

$\sum_{\alpha_{k}=1}^{A_{k}} \sum_{j=1}^{S}\left(x_{k j \alpha_{k} t}^{1}+x_{k j \alpha_{k} t}^{2}\right) \geq \sum_{i=1}^{k-1} \sum_{\alpha_{i}=1}^{A_{i}} \sum_{j=1}^{S}\left(x_{i j \alpha_{i} t}^{1}+x_{i j j_{i} t}^{2}\right)$ Relation $_{i k} \quad \forall k(k>1), t$

BOM relationship (6.4)

$x_{i j \alpha_{i} t}^{1} \leq$ Capacity $_{i j \alpha_{i} t}^{1}$

$x_{i j \alpha_{i} t}^{1} \geq$ Capacity $_{i j \alpha_{i} t}^{1} a_{i j \alpha_{i} t}$

$\forall i, j, \alpha_{i}, t$

$x_{i j \alpha_{i} t}^{2} \leq$ Capacity $_{i j \alpha_{i} t}^{2} a_{i j \alpha_{i} t}$

$\forall i, j, \alpha_{i}, t$

$\forall i, j, \alpha_{i}, t$

constraint

$\sum_{j=1}^{S} x_{i j \alpha_{i} t}^{1} \leq \pi_{i \alpha_{i} t} \operatorname{Mcap}_{i \alpha_{i} t}$

$\forall i, \alpha_{i}, t$

$\sum_{\alpha_{i}=1}^{A_{i}} \pi_{i \alpha_{i} t}=1$

$\forall i, t$

$v_{t}=\sum_{i=1}^{P} \sum_{\alpha_{i}=1}^{A_{i}} \pi_{i \alpha_{i}} v a_{i \alpha_{i} t}$

$\forall t$

Supplier capacity and economies of

scale constraints

$y_{j l}\left(\right.$ Capacity $_{k l \alpha_{k} t}^{1}+$ Capacity $\left._{k l \alpha_{k} t}^{2}\right) \geq u_{j k l \alpha_{k} t}$

$\forall j, k, l, \alpha_{k}, t$

Product design selection and product value calculation constraints

$\sum_{j=1}^{S} u_{j k l \alpha_{k} t} \leq x_{k l \alpha_{k} t}^{1}+x_{k l \alpha_{k} t}^{2}$

$\forall k, l, \alpha_{k}, t$

Supply chain network constraint

$\sum_{\alpha_{k}=1}^{A_{k}} \sum_{l=1}^{S} u_{j k l \alpha_{k} t} \geq$ Relation $_{i k} \sum_{\alpha_{i}=1}^{A_{i}}\left(x_{i j \alpha_{i} t}^{1}+x_{i j \alpha_{i} t}^{2}\right) \quad \forall i, j, k, t \mid$ Relation $\left._{i k}>0\right\}$

Transportation

ptime $_{i t} \geq p t_{i j \alpha_{i} t}\left(\frac{x_{i j \alpha_{i} t}^{1}}{\text { Capacity }_{i j \alpha_{i} t}^{1}}+\frac{x_{i j \alpha_{i} t}^{2}}{\text { Capacity }_{i j \alpha_{i} t}^{2}}\right)$

$L T_{i t} \geq L T_{k t}$ Relation $_{i k}+$ ptime $_{i t}$

$L T_{i t} \geq$ ptime $_{i t}$

$L T_{1 t}=\sum_{n=0}^{N} 2^{n} \gamma_{n t}$

$\left.\begin{array}{l}\forall i, j, \alpha_{i}, t \mid \text { Capacity }_{i j \alpha t}^{1}>0 \\ \forall i, k, t \mid \text { Relation }_{i k}>0 \\ \forall i, t \mid \text { Relation }_{i k}=0 \text { for } \forall k>i\end{array}\right\} \begin{aligned} & \text { Lead time } \\ & \text { calculation } \\ & \text { constraints }\end{aligned}$

$\delta_{n t} \leq$ Demand $_{1 t}$

$\forall t$

$\forall t, n \in[0, \mathrm{~N}]$

$\delta_{n t} \leq M d e m \times \gamma_{n t}$

$\forall t, n \in[0, \mathrm{~N}]$

$\forall t, n \in[0, \mathrm{~N}]$

Lead time - 


$$
\begin{aligned}
& \phi_{1 t}+\phi_{2 t} \leq 1 \\
& \forall t \\
& \lambda_{q t} \leq \phi_{q t} \\
& \forall t, q \in\{1,2\} \\
& \lambda_{q t} \leq v_{t} \\
& \phi_{q t}+v_{t} \leq \lambda_{q t}+1 \\
& \forall t, q \in\{1,2\} \\
& \tau_{t}^{+} \sum_{\alpha_{1}=1}^{A_{1}} \operatorname{Mcap}_{1 \alpha_{1} t} \geq \sum_{\alpha_{1}=1}^{A_{1}} \sum_{j=1}^{S}\left(x_{1 j \alpha_{1} t}^{1}+x_{1 j \alpha_{1} t}^{2}\right)-\text { Demand }_{1 t} \quad \forall t \\
& \tau_{t}^{-} \text {Mdem } \geq \text { Demand }_{1 t}-\sum_{\alpha_{1}=1}^{A_{1}} \sum_{j=1}^{S}\left(x_{1 j \alpha_{1} t}^{1}+x_{1 j \alpha_{1} t}^{2}\right) \quad \forall t \\
& \tau_{t}^{+}+\tau_{t}^{-} \leq 1 \quad \forall t \\
& k_{t}^{+} \leq \text {Demand }_{1 t} \quad \forall t \\
& k_{t}^{+} \leq \tau_{t}^{+} \text {Mdem } \quad \forall t \\
& \text { Demand }_{1 t}+\tau_{t}^{+} \text {Mdem } \leq k_{t}^{+}+\text {Mdem } \quad \forall t \\
& k_{t}^{-} \leq \sum_{\alpha_{1}=1}^{A_{1}} \sum_{j=1}^{S}\left(x_{1 j \alpha_{1} t}^{1}+x_{1 j \alpha_{1} t}^{2}\right) \\
& \forall t, q \in\{1,2\} \\
& \forall t \\
& k_{t}^{-} \leq \tau_{t}^{-} \sum_{\alpha_{1}=1}^{A_{1}} \operatorname{Mcap}_{1 \alpha_{1} t} \quad \forall t \\
& \sum_{\alpha_{1}=1}^{A_{1}} \sum_{j=1}^{S}\left(x_{1 j \alpha_{1} t}^{1}+x_{1 j \alpha_{1} t}^{2}\right)+\tau_{t}^{-} \sum_{\alpha_{1}=1}^{A_{1}} \operatorname{Mcap}_{1 \alpha_{1} t} \leq k_{t}^{-}+\sum_{\alpha_{1}=1}^{A_{1}} \operatorname{Mcap}_{1 \alpha_{1} t} \quad \forall t \\
& \psi_{1 t} \leq k_{t}^{+} \\
& \forall t \\
& \psi_{1 t} \leq \phi_{1 t} \text { Mdem } \\
& \forall t \\
& \phi_{1 t} M d e m+k_{t}^{+} \leq \psi_{1 t}+\text { Mdem } \quad \forall t \\
& \psi_{2 t} \leq k_{t}^{+} \\
& \forall t \\
& \psi_{2 t} \leq \phi_{2 t} \text { Mdem } \\
& \forall t \\
& \phi_{2 t} M d e m+k_{t}^{+} \leq \psi_{2 t}+\text { Mdem } \quad \forall t \\
& \psi_{3 t} \leq k_{t}^{-} \quad \forall t \\
& \psi_{3 t} \leq \phi_{1 t} \sum_{\alpha_{1}=1}^{A_{1}} \operatorname{Mcap}_{1 \alpha_{1} t} \quad \forall t \\
& \phi_{1 t} \sum_{\alpha_{1}=1}^{A_{1}} \operatorname{Mcap}_{1 \alpha_{1} t}+k_{t}^{-} \leq \psi_{3 t}+\sum_{\alpha_{1}=1}^{A_{1}} \operatorname{Mcap}_{1 \alpha_{1} t} \quad \forall t \\
& \psi_{4 t} \leq k_{t}^{-} \quad \forall t \\
& \psi_{4 t} \leq \phi_{2 t} \sum_{\alpha_{1}=1}^{A_{1}} \operatorname{Mcap}_{1 \alpha_{1} t} \quad \forall t \\
& \phi_{2 t} \sum_{\alpha_{1}=1}^{A_{1}} \operatorname{Mcap}_{1 \alpha_{1} t}+k_{t}^{-} \leq \psi_{4 t}+\sum_{\alpha_{1}=1}^{A_{1}} \operatorname{Mcap}_{1 \alpha_{1} t} \quad \forall t \\
& x_{i j \alpha_{i} t}^{1}, x_{i j \alpha_{i} t}^{2}, u_{j k l \alpha_{k} t}, L T_{i t}, \text { ptime }_{i t}, \lambda_{1 t}, \lambda_{2 t}, \psi_{1 t}, \psi_{2 t}, \psi_{3 t}, \psi_{4 t}, k_{t}^{+}, k_{t}^{-}, \text {Demand }_{k t}, v_{t}, \delta_{n t} \geq 0 \\
& \pi_{i \alpha_{i} t}, y_{j l}, a_{i j \alpha_{i} t}, \tau_{t}^{+}, \tau_{t}^{-}, \phi_{1 t}, \phi_{2 t}, \gamma_{n t} \in\{0,1\}
\end{aligned}
$$


Equation (6.2) calculates the demand of the final product (component 1) for each time period based on selected component design alternatives, price, and the time period adjustment parameter. The original demand function in non-linear form is given below.

$$
\text { Demand }_{1 t}=\left(\beta_{1} \text { price }_{t}^{2}+\beta_{2}\right) \times v_{t} \times \text { timemultiplier }_{t}
$$

Since equation (7.1) is non-linear due to multiplication of the price and $v$ variables, a linearization schema is presented in Appendix section A.2. Equation (6.3) does not necessarily constrain the feasible region, but instead is used to evaluate the demand for each selected component alternative design based on the demand for the final product and Bill of Materials data. Similarly, equation (6.4) makes sure that each component that is assembled into another one is manufactured in at least an amount required by the Bill of Materials data. The difference between these two equations is that equation (6.4) constrains the minimum manufacturing quantity of a component where equation (6.3) only calculates the demand for this component but does not enforce any limits on the manufacturing amounts. Equations (6.5), (6.6), and (6.7) establish supplier capacity limits and an economies of scale structure as explained in preliminary models. The next three constraints incorporate the product design decisions into the model. The first constraint (6.8) ensures that only selected component alternative designs (for which $\pi_{i \alpha_{i} t}=1$ ) are manufactured. The second product design constraint (6.9) requires that exactly one component design alternative is selected for each component in each time period. The last product design constraint (6.10) is used to calculate total value of the final product based on component design alternative selections. The supply chain network design is set up by the equation (6.11) by making sure that two suppliers are linked (that is $y_{j l}=1$ ) if any transportation occurs between them (that is $u_{j k l \alpha_{k} t}>0$ ) for a component. Equation (6.12) guarantees that the total amount of a component transported from a supplier is at most the manufactured quantity by 
this supplier. The next constraint (6.13) makes sure that for a component manufactured at a certain supplier, all required subcomponents are transported to this supplier from other suppliers. Equations (6.14) through (6.17) are used to calculate the lead times. Equation (6.14), similar to the equation (3.6) described in the preliminary models, makes sure that the production time of a component is at least equal to the longest time it takes for manufacturing this component among all the suppliers that manufacture this particular component. This constraint is necessary since it is assumed that the production of a component starts only after all of its subcomponents are delivered to the manufacturer. Therefore, the longest production time among all of a component's suppliers is defined as the production time of this component. However, different from the preliminary modeling, in this complete model it is assumed that production time data $\left(p t_{i j \alpha_{i}}\right)$ is given for the total capacity of the supplier, hence the final production time of a component at a particular supplier is determined relative to the capacity utilization of this supplier. For example, if a supplier states that it takes 10 days to manufacture the complete batch in full capacity utilization, then the model decides that the production time of this component at this supplier would be 5 days if half the capacity is used. Equations (6.15) and (6.16) are used to calculate the final lead time of a component in the same fashion described in the preliminary models where the former constraint (only defined for components which have subcomponents) ensures that the lead time of a component is equal to the summation of its own manufacturing time and the maximum lead time of its subcomponents. The latter constraint is used only for the components which do not have any subcomponents to make sure that their final lead time is at least equal to their production times. In the lead time constraints set, the last equation (6.17) is used to represent the final lead time of the main product with binary variables for inventory cost linearization described in Appendix section A.3. 
Constraints (6.18) through (6.45) are used for linearization of the model and do not impose any actual limits on the product and supply chain design. Equations (6.18), (6.19), and (6.20) ensure that $\delta_{n t}$ variables take values to truly represent demand - lead time multiplication in a binary format. Similarly, while equation (6.21) ensures that only one of the second or third price levels is selected, the following three equations ((6.22), (6.23), and (6.24)) ensure that the $\lambda_{1 t}$ and $\lambda_{2 t}$ variables take correct values to capture price and product design value multiplications.

In order to calculate the satisfied demand, which is the minimum of demand and total production, equations (6.25), (6.26), and (6.27) control the $\tau_{t}^{+}$and $\tau_{t}^{-}$variable values to represent whether the demand or the total production is larger. Based on these $\tau_{t}^{+}$and $\tau_{t}^{-}$values, $k_{t}^{+}$and $k_{t}^{-}$ variables take the minimum of the demand or the total production via equations (6.28) through (6.33). The following ten constraint sets, equations (6.36) through (6.45), control the $\psi$ variables that are used in revenue calculation and described in revenue linearization in section A.1 of the Appendix. Finally, equation (6.46) establishes the required variable types and bounds.

This complete DFSC model formulation defines all of the considered problem aspects under the stated assumptions. Therefore, this model is used as the base formulation to investigate research questions. However, this research not only investigates the proposed DFSC concept but it also focuses on different modeling practices and the tradeoffs between solution performance and model features. As the complete DFSC model is very large and complex, despite the simplification by linearization of the model, it requires too much computational time and power to optimize. In addition, this model entails several parameters that sometimes companies cannot estimate easily. Therefore, different reduced models are developed to investigate the impacts of certain concepts on the value of the model and solution time and quality. The following section (3.4) provides different models that are derived from the developed complete DFSC model. 


\subsection{REDUCED MODELS}

As stated in the research question 1, this research aims to investigate the importance of different product design and supply chain performance metrics in real industry cases as well as their impacts on modeling the DFSC problems and impacts on the performance of the solution techniques. Therefore, to investigate impacts of different performance metrics, different reduced DFSC models are developed. These models are based on the developed complete DFSC model and they are developed by removing some model features, changing some assumptions, or both. These models are presented below and the differences from the complete model are explained. The impacts of these changes on the validation of the model and performance of the solution techniques are discussed in sections 5.2 and 5.3.1, respectively.

Initially, the economies of scale concept is removed from the complete model resulting in the reduced DFSC model 1. Although this is a widely used concept both in industry and academia in supply chain modeling, for simplification purposes, it is usually modeled by using piecewise linear functions with only a couple of segments as in the complete DFSC model. Therefore, the actual representation of the economies of scale concept is often only an approximation in order to keep the models linear. In addition, the unit manufacturing cost data is often estimated for a long-term planning in such models thus it involves a significant amount of uncertainty. Moreover, demanding more detailed cost data based on the capacity utilization of the suppliers only amplifies this uncertainty. Briefly, the first reduced model investigates the impacts of removing the economies of scale concept from the DFSC modeling. It is assumed that using an average unit manufacturing cost (thus having a less realistic model) is a reasonable trade off for the increased uncertainty due to more detailed data requirements for the economies of scale modeling. 
In the complete DFSC model, the economies of scale were introduced by dividing the supplier capacities into two different levels and using an associated two-level unit manufacturing cost structure. This representation drives constraints given in equations (6.6) and (6.7) and also requires the introduction of a binary variable, $a_{i j \alpha_{i} t}$, to control the proper application of the concept. As a result, removing the economies of scale concept eliminates these two constraints and the corresponding binary variable. In addition, since the production quantities at each supplier are no longer divided into two levels, the second level manufacturing quantity variable, $x_{i j \alpha_{i}{ }^{2}}{ }^{2}$, and the second level capacity parameter, Capacity ${ }_{i j j_{i}{ }^{2}}{ }^{2}$, are no longer necessary. As it is discussed in the model asymptotic size analysis section (3.5), these changes significantly reduce the number of variables and constraints. The mathematical formulation of the reduced DFSC model - 1 is given in Appendix B.1.

In the reduced DFSC model 2, the impacts of removing the lead time (and thus inventory) from the complete DFSC model are investigated. This reduced model is based on the complete DFSC model therefore it utilizes the economies of scale concept. When the lead time concept is removed, only the direct cost related supply chain performance metrics remain in the model. As it is discussed in the model validation section (5.2), the lead time data is often hard to collect or estimate compared to the cost data. In addition, supply chain costs are usually determined and fixed for a period through contractual agreements where the lead time depends on the actual daily operations and varies greatly. Although contracts may oblige supply chain partners to perform within certain lead time limits via penalties or other sanctions, it is still very hard for companies to estimate a true lead time for a supply chain in the long-term. Furthermore, the inventory levels highly depend on the inventory keeping and product ordering policies. The variability of the lead time and demand also requires certain assumptions as discussed in the 
complete model formulation section (3.3) such as constant coefficient of variation and linearly changing average inventory levels. Therefore, it may be preferable to neglect inventory and lead time concepts in the initial planning stage and consider them separately in a later phase of the supply chain planning process to save from the model solution time and achieve the optimal solution. The impacts of removing the inventory and lead time concepts on the model and solution quality are discussed in section 5.3.1 in detail. The mathematical formulation of the reduced DFSC model - 2 is given in Appendix B.2.

In the complete DFSC model, the lead time and inventory cost concepts require several binary $\left(\gamma_{n t}, \delta_{\mathrm{nt}}\right)$ and continuous $\left(L T_{i t}\right.$, ptime $\left._{i t}\right)$ variables. In addition, removing lead time and inventory concepts makes the related parameters $\left(p t_{i j \alpha_{i} t}, h_{t}\right.$, periodlength $\left.h_{t}, z_{s s r a t i o}, \rho_{1}, \rho_{2}\right)$ redundant. Finally, constraints (6.14) through (6.20) and the inventory cost calculation in the objective function, which is given in equation (5.5), can be removed from the model. The significance of the removal of these variables and constraints are discussed in section 3.5.

\subsection{MODEL ASYMPTOTIC SIZE ANALYSIS}

In this section, an asymptotic size analysis of the preliminary, complete, and reduced models is provided. This analysis summarizes the changes in the number of variables and number of constraints, as well as the expected performance of the solution techniques.

\section{Preliminary Models}

The complexity of the proposed models increases not only by the number of product components and suppliers, but also with the interactions between them. For the cost minimization model, the number of variables is equal to $4(P S)+S^{2}$, where $P$ is the number of components and $S$ is the 
number of total suppliers. $2 P S$ of these variables are binary, where the rest are non-negative continuous variables. The number of supply chain generation constraints depends on the direct relationships between the components therefore only an upper bound can be calculated. The maximum number of total constraints is equal to $P\left(P S^{2}+3 S+1\right)$.

For the lead time minimization model, the number of variables is equal to $4 P S+2 P$, where $2 P S$ of these variables are binary. The number of constraints is based on the relationships between the components, and between components and suppliers. Therefore, an upper bound for the total number of constraints is $P(P+4 S+2)$.

As shown above for both models, the number of variables and constraints increase significantly when the number of components or suppliers is increased.

\section{Complete DFSC Model}

Among the developed models, the complete model captures all the described concepts and assumptions, making it the most complex model in terms of variables, constraints, and their interactions. By using the model's set notation, the total number of the variables in the complete DFSC model can be expressed as $S^{2}+T\left(13+2 N+3 P+\left[\left(P A_{i}\right)\left(1+3 S+S^{2}\right)\right]\right)$, where $T$ is number of time periods, $A_{i}$ is total number of design alternatives for the component $i$, and $N$ is number of lead time - demand binary variables. Therefore, the number of variables in this model increases linearly with the number of components, number of time periods, number of lead time - demand binary variables, and number of design alternatives per component when one of them is increased and the others are constant. However, the number of variables increases with the square of the number of available suppliers when the number of other variables is constant; hence this parameter has the most significant impact on the model size. $S^{2}+T\left(4+N+\left[\left(P A_{i}\right)(1+S)\right]\right)$ of these variables are binary and this shows that the number of 
available suppliers is critical as the binary variables increase the solution time significantly more

than continuous variables. It should be noted that among all variables, the number of $x_{i j \alpha_{i}{ }^{t}}, x_{i j j_{i}{ }^{2}}$, $u_{j k l \alpha_{k} t}, \pi_{i \alpha_{i} t}, y_{j l}$, and $a_{i j \alpha_{i} t}$ variables, which were described in section 3.3, highly depends on the model set sizes and their interactions, where the number of $u_{j k l a_{k} t}$ and $y_{j l}$ variables increases with the square of the number of available suppliers, significantly increasing the model size.

Similar to the preliminary models, the exact number of constraints in the complete DFSC model depends on the parameter values which define component - supplier relationships, Bill of Material information, and component design alternatives. An upper bound on the constraints is equal to $T\left(30+3 N+P\left[4+P+P S+A_{i}\left(1+5 S+S^{2}\right)\right]\right)$, which suggests that the total number of constraints significantly depends on the number of time periods. This is due to the structure of the model to capture the total life cycle of the product based on life cycle phases rather than using short time periods for tactical or operational planning.

The model analysis suggests that the complete DFSC model size highly depends not only on the size of the parameter sets but also on the interactions between these sets. Therefore, each problem instance differs by size and complexity and larger problems may have significantly fewer constraints due to relatively simpler Bill of Materials or component - supplier interactions.

\section{Reduced Models}

In the first reduced model, where the economies of scale concept is removed, the total number of variables is reduced by $2 P S A_{i} T$ to $S^{2}+T\left(13+2 N+3 P+\left[\left(P A_{i}\right)\left(1+S+S^{2}\right)\right]\right)$. The significance of this reduction is from the fact that $P S A_{i} T$ of these variables are binary, promising a significant reduction in solution times. In addition, with the removal of the economies of scale concept, the upper limit on the total number of constraints is reduced by $2 P S A_{i} T$ due to elimination of 
equations (6.6) and (6.7). It should be noted that although the reduction in the number of constraints is important, having fewer binary variables notably reduces the solution space.

With the exclusion of lead time and inventory cost concepts, the second reduced model employs $2 T(P+N)$ less variables than the complete DFSC model. This reduction eliminates a total of $T N$ binary variables $\left(\gamma_{n t}\right)$ which is less than the binary variable reduction in the first reduced model. In terms of constraint reduction, the second reduced model cuts $T\left(3 N+P+P^{2}+P S A_{i}\right)$ constraints by purging equations (6.14) through (6.20). The total reduction in the number of constraints is less than the same reduction in the first reduced model provided that the $P<S A_{i}$ condition is true. It should be noted that this condition would hold in almost all real cases unless the majority of the considered components are manufactured by a very small set of suppliers. This analysis suggests that the first reduced model reduces more variables and constraints suggesting a shorter solution time as compared to the second reduced model. Section 5.3.1 specifically addresses the solution quality and time performances of the reduced models.

Table 2 summarizes the total number of variables and maximum number of constraints possible for each developed model. 
Table 2: Summary of number of variables and constraints in different models

\begin{tabular}{|c|c|c|c|}
\hline & $\begin{array}{l}\text { Number of } \\
\text { Variables }\end{array}$ & $\begin{array}{c}\text { Number of Integer } \\
\text { Variables }\end{array}$ & Number of Constraints \\
\hline \multicolumn{4}{|l|}{ Preliminary Models } \\
\hline $\begin{array}{l}\text { Preliminary cost } \\
\text { minimization model }\end{array}$ & $4 \mathrm{PS}+\mathrm{S}^{2}$ & $2 \mathrm{PS}+\mathrm{S}^{2}$ & $\mathrm{P}\left(\mathrm{PS}^{2}+3 \mathrm{~S}+1\right)$ \\
\hline $\begin{array}{l}\text { Preliminary lead time } \\
\text { minimization model }\end{array}$ & $4 \mathrm{PS}+2 \mathrm{P}$ & 2PS & $\mathrm{P}(\mathrm{P}+4 \mathrm{~S}+2)$ \\
\hline $\begin{array}{l}\text { Preliminary reduced cost } \\
\text { minimization model }\end{array}$ & 4PS & 2PS & $3 \mathrm{PS}+\mathrm{P}$ \\
\hline \multicolumn{4}{|l|}{ DFSC MIP Models } \\
\hline Complete DFSC model & $\begin{array}{c}\mathrm{S}^{2}+ \\
\mathrm{T}(13+2 \mathrm{~N}+3 \mathrm{P}+ \\
\left.\left[\left(\mathrm{PA}_{\mathrm{i}}\right)\left(1+3 \mathrm{~S}+\mathrm{S}^{2}\right)\right]\right)\end{array}$ & $\begin{array}{l}\mathrm{S}^{2}+\mathrm{T}(4+\mathrm{N}+ \\
\left.\mathrm{PA}_{\mathrm{i}}[1+\mathrm{S}]\right)\end{array}$ & $\begin{array}{c}30 \mathrm{~T}+3 \mathrm{NT}+ \\
\mathrm{PT}\left[4+\mathrm{P}+\mathrm{PS}+\mathrm{A}_{\mathrm{i}}\left(1+5 \mathrm{~S}+\mathrm{S}^{2}\right)\right]\end{array}$ \\
\hline Reduced DFSC model - 1 & $\begin{array}{c}\mathrm{S}^{2}+ \\
\mathrm{T}(13+2 \mathrm{~N}+3 \mathrm{P}+ \\
\left.\left[\left(\mathrm{PA}_{\mathrm{i}}\right)\left(1+\mathrm{S}+\mathrm{S}^{2}\right)\right]\right)\end{array}$ & $\begin{array}{c}\mathrm{S}^{2}+ \\
\mathrm{T}\left(4+\mathrm{N}+\mathrm{PA}_{\mathrm{i}}\right)\end{array}$ & $\begin{array}{c}30 \mathrm{~T}+3 \mathrm{NT}+ \\
\mathrm{PT}\left[4+\mathrm{P}+\mathrm{PS}+\mathrm{A}_{\mathrm{i}}\left(1+3 \mathrm{~S}+\mathrm{S}^{2}\right)\right]\end{array}$ \\
\hline Reduced DFSC model - 2 & $\begin{array}{c}\mathrm{S}^{2}+ \\
\mathrm{T}(13+\mathrm{P}+ \\
\left.\left[\left(\mathrm{PA}_{\mathrm{i}}\right)\left(1+3 \mathrm{~S}+\mathrm{S}^{2}\right)\right]\right)\end{array}$ & $\begin{array}{c}\mathrm{S}^{2}+\mathrm{T}(4+ \\
\left.\mathrm{PA}_{\mathrm{i}}[1+\mathrm{S}]\right)\end{array}$ & $\begin{array}{c}30 \mathrm{~T}+ \\
\mathrm{PT}\left[3+\mathrm{PS}+\mathrm{A}_{\mathrm{i}}\left(1+4 \mathrm{~S}+\mathrm{S}^{2}\right)\right]\end{array}$ \\
\hline
\end{tabular}

In order to illustrate the significance of the reductions in the number of variables and constraints, Table 3 summarizes these values for all different models based on a problem instance with 10 components, 3 design alternatives for each component, 15 suppliers, 4 time periods, and 8 lead time - demand binary variables. 
Table 3: Numerical illustration of model asymptotic size of different models

\begin{tabular}{lccc}
\hline & $\begin{array}{c}\text { Number of } \\
\text { Variables }\end{array}$ & $\begin{array}{c}\text { Number of } \\
\text { Integer Variables }\end{array}$ & Number of Constraints \\
\hline Preliminary Models & & 525 & 22,960 \\
$\begin{array}{l}\text { Preliminary cost } \\
\text { minimization model }\end{array}$ & 825 & 300 & 720 \\
$\begin{array}{l}\text { Preliminary lead time } \\
\text { minimization model }\end{array}$ & 620 & 300 & 460 \\
$\begin{array}{l}\text { Preliminary reduced cost } \\
\text { minimization model }\end{array}$ & 600 & & 42,896 \\
\hline DFSC MIP Models & & 2,193 & 39,296 \\
\hline Complete DFSC model & 32,981 & 393 & 40,560 \\
Reduced DFSC model - 1 & 29,381 & 2,161 & \\
\hline Reduced DFSC model -2 & 32,837 & & \\
\hline
\end{tabular}

This numerical illustration presents a $10.92 \%$ reduction in number of variables for the reduced DFSC model -1 and $0.44 \%$ reduction for the second reduced DFSC model. More importantly, the reduction in the number of binary variables, which challenge and lengthen the solution procedures, is 1,800 for the first reduced model and 32 for the second reduced model. 


\subsection{SOLUTION METHODOLOGY}

In order to solve the developed MIP models, a deterministic optimization method (branch and bound) and different metaheuristics are investigated. This chapter provides a brief history of these methods followed by the details of specific implementations for this research. Finally, their performance test results on the preliminary test models are presented.

\subsection{DETERMINISTIC OPTIMIZATION METHODOLOGIES}

\subsubsection{Overview}

Deterministic optimization methodologies include all varieties of solution techniques that are aimed toward solving the problems that only involve deterministic parameters and variables. Problem formulations that only include continuous variables and linear functions are solved by using Linear Programming (LP), which was mainly developed during World War II and evolved by incorporation of fast and optimal guaranteed solution methods such as the Simplex Algorithm. For a problem to be formulated as an LP, it should satisfy four assumptions [63]. The first one is the linearity assumption that states each of the variable's contribution to any equation in the model is constant and these contributions are additive, therefore prohibiting any interactions (including multiplication and division) of the variables. The second assumption 
(divisibility) states that all of the variables in the model are continuous. The third assumption is certainty which requires all the model parameters are to be known constants, thus it assumes that the model is solved under perfect information. The final assumption is that the model has a single objective which is optimized under the other assumptions. However, many real problem cases violate the divisibility assumption by requiring either all or some of the variables to take integer values. When all of a model's variables are required to take integer values, this model formulation is called Integer Programming (IP). On the other hand, when models engage both continuous and integer variables, they are named as Mixed Integer Programming formulations.

The MIP problems are usually very hard to solve effectively compared to the LP problems. However, certain methods are developed to accelerate the solution procedures (e.g., branch and bound, cutting plane, branch and cut, delayed column generation) and save time over the enumeration of all possible integer solutions. Since this research does not evaluate nor use all of these methods, only the branch and cut method is discussed in detail. The branch and cut method is actually a combination of branch and bound and cutting plane methods. The branch and bound method, first introduced by Land and Doig [38] in 1960, aims to shrink the search space for the problem by pruning unpromising search space regions thus saving computational time. The underlying process of this method is that after the initial LP relaxation of the problem is solved, the solution space is divided into smaller sub-solution spaces (branching). During this process, a solution tree is constructed to keep track of which solutions (nodes) have which variables at the integer values and the objective values of these solutions. Within the process, the time savings come from pruning the unpromising branches of the tree instead of spending time on these solutions as in the complete enumeration. By keeping track of each node's best possible solution value, as an upper (or lower) bound, other nodes that have worse bounds can be 
removed from the search space. The cutting plane method, first introduced by Gomory [28] in 1963, aims to add new specially structured constraints (cuts) to the original model in order to eliminate the non-integer optimal solution for the initial model relaxation. These cuts are constructed in a special formation so that they do not remove any feasible integer solutions from the search space when eliminating the current non-integer solution. The branch and cut method used in this research is a combination of branch and bound and cutting plane methods, where during the branch and bound algorithm, relevant cuts are introduced to further reduce the solution space size.

The most significant feature of solving LP and MIP problems by the mentioned methods is that finding the optimal solutions to these problems is guaranteed, provided one exists. However, MIP problems are usually very hard to solve to the optimal value due to the drastically increasing solution space size for larger problems. In this case, the specially structured solution procedures such as heuristics and metaheuristics need to be considered. Their details are discussed in section 4.2. The following section provides the computational test results of the described MIP branch and bound solution procedure on the preliminary models.

\subsubsection{Performance on the Preliminary Models}

In order to perform tests on the performance of MIP and metaheuristics, five different illustrative problem instances are generated randomly for the preliminary models. Table 4 shows detailed information about each of these problem instances. 
Table 4: Illustrative test problem data for the preliminary models

\begin{tabular}{cccc}
\hline $\begin{array}{c}\text { Problem } \\
\text { Instance }\end{array}$ & $\begin{array}{c}\text { Number of } \\
\text { Components }\end{array}$ & $\begin{array}{c}\text { Number of } \\
\text { Suppliers }\end{array}$ & $\begin{array}{c}\text { Number of } \\
\text { Components per } \\
\text { Supplier }\end{array}$ \\
\hline 1 & 5 & 10 & $1-3$ \\
2 & 10 & 20 & $1-4$ \\
3 & 25 & 50 & $1-7$ \\
4 & 50 & 100 & $1-14$ \\
5 & 100 & 200 & $1-19$ \\
\hline
\end{tabular}

For all instances, unit manufacturing costs (that suppliers charge per component produced) are selected randomly based on a uniform distribution (here to referred as uniform-randomly) from a [10-100] interval for the first production level and from a [1-70] interval for the second production level. However, the second level cost is forced to be less than or equal to the first level cost to ensure the economies of scale concept is considered. Similarly, first and second level capacity values for each component-supplier pair are selected uniform-randomly having the second level capacity larger than or equal to the first level capacity. For all instances, lead times are distributed uniform-randomly from a [100-1,000] interval. SC network costs are again selected uniform-randomly from a $[10,000-1,000,000]$ interval.

The MIP solution technique is utilized by using the CPLEX $^{\circledR}$ optimization software to optimize each model separately. CPLEX ${ }^{\circledR}$ uses a robust branch and cut algorithm to solve MIP models optimally in a short time period, however MIP models tend to increase in size significantly as the number of variables increases making the solution procedure very time consuming. The behavior of the MIP solution techniques for small and large models is tested and the results and the performance of the solution procedure is analyzed.

All CPLEX ${ }^{\circledR}$ runs are completed on the Microsoft Windows ${ }^{\circledR}$ XP platform on a computer with an Intel ${ }^{\circledR}$ Pentium $43.06 \mathrm{GHz}$ CPU and $1 \mathrm{~GB}$ of RAM. 
The cost minimization and lead time minimization models are solved by using default $\mathrm{CPLEX}^{\circledR}$ parameters as the base case. Table 5 shows the results of the cost minimization runs where Table 6 reports results of the lead time minimization runs. Problem instance 4 in the cost minimization model and problem instance 5 in the lead time minimization model cannot be solved to the optimal value due to memory limitations. Therefore, the best found integer solutions are reported.

Table 5: MIP cost minimization model preliminary run results

\begin{tabular}{cccc}
\hline $\begin{array}{c}\text { Problem } \\
\text { Instance }\end{array}$ & $\begin{array}{c}\text { Final Integer } \\
\text { Solution (\$) }\end{array}$ & $\begin{array}{c}\text { Relative } \\
\text { Optimality Gap* }\end{array}$ & $\begin{array}{c}\text { Solution Time } \\
\text { (in seconds) }\end{array}$ \\
\hline 1 & $21,769,159$ & $0.00 \%$ & 0.11 \\
2 & $56,899,401$ & $0.00 \%$ & 0.21 \\
3 & $46,931,986$ & $0.00 \%$ & $2,457.76$ \\
4 & $81,096,271$ & $16.18 \%$ & $494,246.30$ \\
5 & $381,543,320$ & $0.00 \%$ & $113,737.19$ \\
\hline
\end{tabular}

* Relative optimality gap is the percentage of the gap between the best known integer solution and the best possible integer solution (a lower bound).

Table 6: MIP lead time minimization model preliminary run results

\begin{tabular}{cccc}
\hline $\begin{array}{c}\text { Problem } \\
\text { Instance }\end{array}$ & $\begin{array}{c}\text { Final Integer } \\
\text { Solution (hr.) }\end{array}$ & $\begin{array}{c}\text { Relative } \\
\text { Optimality Gap* }\end{array}$ & $\begin{array}{c}\text { Solution Time } \\
\text { (in seconds) }\end{array}$ \\
\hline 1 & 2,704 & $0.00 \%$ & 0.13 \\
2 & 3,381 & $0.00 \%$ & 0.13 \\
3 & 1,695 & $0.00 \%$ & 5.59 \\
4 & 2,193 & $0.00 \%$ & 228.69 \\
5 & 3,108 & $11.03 \%$ & $102,465.21$ \\
\hline
\end{tabular}

* Relative optimality gap is the percentage of the gap between the best known integer solution and the best possible integer solution (a lower bound).

The MIP results show that as the problem size gets larger, the solution time increases significantly. The time increase is more significant in the cost minimization model due to its high dependency on the relationships between the components and suppliers as described in the model 
complexity section. However, the cost minimization model results show that both the solution quality and the solution times depend on the exact problem instance values in addition to the problem instance size. For example, problem instance 4 cannot be solved to the optimal value in more than 5 days and is stopped due to memory limitations where the largest problem instance (5) can be solved to the optimal point in 1.5 days.

\subsection{HEURISTICS AND META HEURISTICS}

As specified before, the time to solve a MIP problem with branch and cut method increases significantly as the problem size gets larger. In this case, heuristics and metaheuristics present an important alternative to the deterministic optimization methods.

\subsubsection{Overview}

Heuristics, in general, can be defined as special algorithms which are designed to solve a problem much faster than the traditional optimization methods, occasionally by guaranteeing an optimal solution or sometimes by providing an approximation limit to the real optimal solution. It should be noted that this approximation limit may be infinite, meaning that no guaranteed optimality approximation is provided by the heuristic. In this sense, heuristics are often designed specifically for a problem (and formulation) and they exploit the problem structure to efficiently come up with good solutions in a shorter time (e.g., Nearest Neighborhood Algorithm for Traveling Salesman Problems and Dijkstra's Algorithm for Shortest Path Problems). However, this special design of the algorithm for a particular problem limits its applicability on other 
problems. In addition, some heuristics are not always developed to optimize a problem but to find a feasible solution very quickly. Especially for large combinatorial optimization problems, MIP techniques may stall even before finding a feasible solution, encouraging the use of heuristics.

Metaheuristics, alternatively, are special types of heuristics that are applicable to a wide range of problems and they are not limited by the problem structure and formulation. However, metaheuristics often utilize some randomness in the search process. In addition, metaheuristics usually move in the search space by utilizing specially designed operations to evaluate a portion of the search space to come up with a good solution quickly. In general, a particular solution quality cannot be guaranteed for metaheuristics. There exist various metaheuristics for different types of problems but the most common ones include Genetic Algorithm (GA), Tabu Search (TS), Simulated Annealing (SA), Ant Colony Optimization (ACO), and Greedy Algorithm (GrA).

The application of different metaheuristics on the supply chain problems are discussed in section 2.3. In this research, GA and TS are selected as candidate metaheuristics for the DFSC problem since they are the commonly used techniques and their performances are known to be very good for these types of problems. Initially, these metaheuristics are evaluated by using the preliminary model formulations. A brief history of these methods and their application details are given below.

\section{Genetic Algorithm}

The Genetic Algorithm was initially developed by Holland and his associates at the University of Michigan in the 1970s [33]. The GA has a biological basis. In brief, the GA imitates the operations of Darwin's Theory of Evolution such as crossover and mutation, to find the optimal 
solution (even though it is not guaranteed). The main idea behind the algorithm is to reach a "good" solution by employing these operations on an initial set of solutions, namely an initial population. The individual solutions, which as a set constitute a population, are called chromosomes. Each chromosome represents a solution for the problem and therefore is encoded to represent a particular solution. Encoding is an important issue for the GA since it dictates how the algorithm advances by limiting the search space and determining the structure of certain GA operators. The chromosomes are often encoded by using binary values for yes - no, open - close and similar decisions or by using integer and/or real numbers to represent real values that the corresponding problem variables could take. Every following generation after the first one is generated by creating new offspring by applying crossover to two selected parent chromosomes from the previous generation and sometimes also letting the best ones live without performing crossover (elitist approach). Mutation is used to partly modify the old generation's individuals or the newly created offspring in order to diverse the search. The GA has certain advantages over other search and optimization methods which are its ability to search the solution space extensively without being stuck on a local optimal point and being computationally beneficial especially for large problem instances. However, its major drawback is that it does not guarantee a global optimal solution which is common for metaheuristics in general. For further detailed information about GA, refer to Goldberg [27].

\section{Tabu Search}

Tabu Search is a metaheuristics solution algorithm that employs a memory structure that defines the way the algorithm moves through the search space. Attributed to Fred Glover [25][26], it was originally developed two decades ago. Since then, several refinements have been made to the algorithm making it more powerful. The main idea of TS is to keep track of previous moves in 
the solution space and diversify the search by encouraging the search process to move to unexplored regions of the solution space by preventing going back to previous steps. This is implemented by a so called "tabu list" which consists of changes made to the solutions in the previous steps. Whenever the algorithm searches for another solution, it is blocked from considering previous solutions as alternatives, thus it is forced to move to an unexplored region of the solution space.

As a first step in a TS procedure, solutions are defined and coded into a solution array similar to the chromosome concept in the GA. The structure of the solution array is very important since it determines the possible operations to be utilized on it. Another important TS concept is "neighborhood" which defines the set of all solutions that lie near the current solution. Each solution is considered to be within the neighborhood of the current solution if it can be reached directly from the current solution by an operation called a "move." Therefore, move and neighborhood are closely related terms and setting one help dictate the other.

In general, the TS algorithm starts by picking a solution randomly from the solution space. After evaluating the objective value for that solution, it evaluates objective values of all feasible solutions within the neighborhood of the current solution and moves to the "best" one in the neighborhood. After a new current solution is fixed, the previous move operation is added into the tabu list thus preventing the algorithm from evaluating the previous solution as a candidate for the next step. As the algorithm moves through the solution space in this manner, the tabu list fills and many moves become prevented. The usual application is to set a limit for the number of tabu moves, so after a certain number of iterations, the oldest move is removed from the list and it is allowed to be examined again. Although the TS algorithm has a tendency to pick a solution with a better objective function value than the current one on every iteration, the 
tabu list may prevent it from doing so. Therefore, the algorithm is sometimes forced to move to a worse solution which helps diversifying the search and preventing being stuck in a local optima.

In addition to the tabu list, which keeps track of last moves thus called "short term memory," some "long term memory" concepts are also popular in the TS. The long term memory generally uses the frequencies of solution segments (attributes), e.g., how many times a solution segment occurs in the search. This type of memory is used to employ "diversification," which aims to force the algorithm to search a wider area by selecting less frequently used attributes or "intensification" which compels the algorithm to focus on a particular region by urging more frequently used attributes. It should be noted that there are many extensions and variations used in TS applications to improve the solution process. For further details of TS concepts, refer to Glover and Laguna [26].

\subsubsection{Performance on the Preliminary Models}

In this research, the GA and TS algorithms are coded in Visual Basic by using Microsoft Visual Studio $^{\circledR} 6.0$ and run on the same hardware as described in MIP performance testing on the preliminary models.

\section{Genetic Algorithm}

In a GA, genes, which are elements within a chromosome, can take different values (called alleles) such as continuous, integer, or binary numbers. They define the structure of a chromosome and therefore have drastic impacts on the behavior, the solution quality, and computational time of the algorithm. In the preliminary testing implementation of the GA, the chromosomes represent the solutions with binary genes. Each chromosome represents a complete solution of the problem where all possible component-supplier pairs are decided to be active (in 
use) or inactive. Each gene of the chromosome corresponds to a component - supplier pair and an allele value ( 1 or 0 ) of the gene represents the status of the corresponding pair; 1, being an active pair and 0 , an inactive pair. Figure 6 depicts an illustrative chromosome structure.

\begin{tabular}{|l|c|c|c|c|c|c|c|c|}
\cline { 2 - 9 } \multicolumn{1}{c|}{} & \multicolumn{8}{c|}{ Component - Supplier Relationship } \\
\cline { 2 - 9 } \multicolumn{1}{c|}{} & $\mathrm{p}_{1}-\mathrm{s}_{1}$ & $\mathrm{p}_{1}-\mathrm{s}_{2}$ & $\mathrm{p}_{2}-\mathrm{s}_{1}$ & $\ldots$ & $\ldots$ & $\mathrm{p}_{\mathrm{p}}-\mathrm{s}_{\mathrm{s}-2}$ & $\mathrm{p}_{\mathrm{p}}-\mathrm{s}_{\mathrm{s}-1}$ & $\mathrm{p}_{\mathrm{p}}-\mathrm{s}_{\mathrm{s}}$ \\
\hline $\begin{array}{l}1=\text { used pair } \\
0=\text { unused pair }\end{array}$ & 1 & 1 & 0 & $\ldots$ & $\ldots$ & 0 & 1 & 0 \\
\hline
\end{tabular}

Figure 6: Chromosome structure of the Genetic Algorithm for the preliminary models

It should be noted that only the possible component - supplier pairs are included in the chromosome structure hence only available suppliers for manufacturing a component are listed under this particular component's section. For example, a chromosome structure for a problem set can be as comp1-supplier2, comp1-supplier5, comp2-supplier3, comp2-supplier6, etc.

The GA solution procedure begins with creation of the initial population. For the next steps, new generations are created by applying two major genetic operators and additional procedures. After copying a certain number of best solutions to the next generation directly (elitist selection rule), new solutions are created by a crossover operator which selects two parents (two solutions from the previous generation) according to a roulette wheel selection rule. In this rule, all solutions have a certain probability of being selected as parents which is distributed according to the objective value of the solution, called the fitness value. Then a cut point is selected randomly based on a uniform distribution (hereafter referred to as uniform-randomly) and the offspring is created by copying the genes from the first parent up to the cut point and copying the rest from the second parent. With a certain probability, these new 
solutions are mutated, which means some genes of the solution are randomly flipped from 1 to 0 or vice versa making any part - supplier pair active or inactive. This mutation procedure adds diversity to the populations and helps prevent being trapped in local optimal solution spaces or early convergence.

Since the defined problem has some constraints, crossover and mutation procedures may lead to infeasible solutions because of their random altering nature. Therefore, a repair procedure, that eliminates the infeasibility of a solution, is employed. Due to the structure of the chromosome and genetic operators, infeasibility can only occur in a segment of a chromosome that corresponds to a single particular part whose genes either come from different parents or are mutated. For that part's chromosome segment, the repair procedure uniform-randomly selects genes to change their value into 1 (to use more suppliers to satisfy the demand) until infeasibility is removed. Although this procedure disturbs the exact replication of parents' structures, it has a small impact in the solutions since only a single segment among many is repaired and major chromosome structures of parents are preserved.

Finally, an immigration concept is employed to ensure the diversity of the population to search a wider solution space and prevent convergence to local optimal solutions. In this procedure, a certain percent of the population is randomly generated instead of being created by using individuals from the previous generation in reference to immigration in nature. If no improvements are observed in the last certain number of generations, the population is "kicked" where after elitist chromosomes are copied, the rest of the population is created randomly similar to the immigration concept. In this case, crossover and mutation operators are not used for the new population. The algorithm terminates either when convergence is determined (very small or no improvements are noted after a certain number of generations) or an iteration limit is reached. 
The preliminary GA application solves for the cost and lead time minimization objectives. The pseudo-code of the GA implementation is given below.

\section{Preliminary GA Pseudo-code:}

Create initial population

Until stopping criteria is met

Copy elitist chromosomes to next generation

Create immigrant chromosomes for the next generation

Until population size is filled

Select two parents according to the roulette wheel rule

Cross subsections of the chromosomes

Decide to mutate or not

If mutate, then randomly perturb the solution; else, continue

Do

Calculate fitness values of the chromosomes

If the best solution is not changed for certain number of generations, then kick population

Do

Check stopping criteria is met, if yes, then terminate; else, continue

The GA requires several parameters to be adjusted before the final testing to reach the maximum performance since the behavior of the GA depends on these parameters. For the preliminary analysis, problem instance 3 with 25 components and 50 suppliers is selected for the parameter adjustment tests since it is not only complex enough to see different results for different parameter settings, but also manageable in size to perform many test runs. The analyzed parameters consist of population size, maximum number of generations, percentage of number of elitist chromosomes, and percentage of immigrating chromosomes. The mutation rate also needs to be adjusted for each run, but a special structure, which starts with lower mutation rates (between 0.01 and 0.2 ) and increases as the algorithm proceeds up to a predetermined high value (between 0.5 and 0.8 ), is used. Although high mutation rates may disturb the similarity of successive generations, initial performance tests suggest increasing mutation rates provides more diversity and helps to prevent local optimal results. In addition to mutation rate changes, another 
operation that reduces the number of active component - supplier pairs is also included into the mutation operation. By this addition, when a new component - supplier pair is activated by the mutation operator, other active pairs for this component are removed keeping the solution feasible. Therefore, mutation rates are not explicitly analyzed within this parameter adjustment test sets. Each parameter setting combination has been run for five times for the parameter tests since the GA is a random algorithm. Performance results are reported in Appendix C. The results suggest that the number of maximum generations has the largest impact on the solution quality (despite increasing computation times) followed by population size. It is intuitive that increasing both the population size and the number of generations results in better solutions and longer computational times since this allows the algorithm to search more solutions in the solution space. However, these test results suggest that increasing the number of generations adds more value to the search than increasing the population size. Therefore, different numbers of generations and population sizes were used for different problem instances, with a preference of increasing the number of generations.

For the percentage of elitist and immigration chromosomes, their impacts on the solution quality is not as significant, although a higher immigration chromosome percentage increases the solution time more than the percentage of elitist chromosomes. This is also intuitive in the sense that immigrating chromosomes are newly created while elitist chromosomes are exact copies from the previous generation, thus it takes more time to create a new feasible solution than copying an existing one. As the final parameter set, the percentage of elitist chromosomes is set at $5 \%$ where the immigration percentage is set at $1 \%$. Table 7 reports results of the GA runs for five preliminary problem instances for the cost minimization problem and Table 8 reports the GA run results for the lead time minimization problem for five preliminary problem instances. 
Table 7: Genetic Algorithm cost minimization model preliminary run results

\begin{tabular}{|c|c|c|c|c|c|c|}
\hline $\begin{array}{l}\text { Problem } \\
\text { Instance }\end{array}$ & $\begin{array}{c}\text { Population } \\
\text { Size }\end{array}$ & $\begin{array}{l}\text { Number of } \\
\text { Generations }\end{array}$ & $\begin{array}{l}\text { Best } \\
\text { Objective } \\
(\$)\end{array}$ & $\begin{array}{l}\text { Solution } \\
\text { time (in } \\
\text { seconds) }\end{array}$ & $\begin{array}{c}\text { Relative } \\
\text { Gap* }\end{array}$ & $\begin{array}{c}\text { Solution Time } \\
\text { Gap** }\end{array}$ \\
\hline 1 & 100 & 50 & $21,769,159$ & 41 & $0.00 \%$ & $99.73 \%$ \\
\hline \multirow{3}{*}{2} & 100 & 50 & $58,098,429$ & 57 & $2.06 \%$ & $99.63 \%$ \\
\hline & 100 & 200 & $58,700,381$ & 249 & $3.07 \%$ & $99.92 \%$ \\
\hline & 200 & 250 & $57,474,011$ & 636 & $1.00 \%$ & $99.97 \%$ \\
\hline \multirow{3}{*}{3} & 100 & 1,000 & $54,389,653$ & 3,389 & $13.71 \%$ & $27.48 \%$ \\
\hline & 100 & 5,000 & $49,263,967$ & 18,966 & $4.73 \%$ & $87.04 \%$ \\
\hline & 200 & 5,000 & $49,553,641$ & 38,692 & $5.29 \%$ & $93.65 \%$ \\
\hline \multirow{3}{*}{4} & 100 & 50 & $144,734,979$ & 614 & $43.97 \%$ & $-80,394.14 \%$ \\
\hline & 200 & 250 & $135,448,500$ & 5,992 & $40.13 \%$ & $-8,148.44 \%$ \\
\hline & 100 & 1,000 & $91,306,584$ & 12,520 & $11.18 \%$ & $3,847.65 \%$ \\
\hline \multirow{3}{*}{5} & 20 & 355 & $474,948,397$ & 3,100 & $19.76 \%$ & $-3,568.94 \%$ \\
\hline & 25 & 1,000 & $414,122,894$ & 11,212 & $7.87 \%$ & $-914.42 \%$ \\
\hline & 100 & 355 & $455,809,970$ & 26,643 & $16.29 \%$ & $-326.89 \%$ \\
\hline \multicolumn{7}{|c|}{$\begin{array}{l}\text { * Relative gap is the percentage of the gap between the best known CPLEX }{ }^{\circledR} \text { solution and the best GA solution. } \\
\text { (Negative values show a better objective function value for the GA.) } \\
\text { ** Solution time gap measures compare how long the CPLEX }{ }^{\circledR} \text { run lasts relative to the GA run. (Negative values } \\
\text { show shorter solution times for the GA.) }\end{array}$} \\
\hline
\end{tabular}

Table 8: Genetic Algorithm lead time minimization model preliminary run results

\begin{tabular}{cccccrr}
\hline $\begin{array}{l}\text { Problem } \\
\text { Instance }\end{array}$ & $\begin{array}{c}\text { Population } \\
\text { Size }\end{array}$ & $\begin{array}{c}\text { Number of } \\
\text { Generations }\end{array}$ & $\begin{array}{c}\text { Best } \\
\text { Objective } \\
\text { (hr) }\end{array}$ & $\begin{array}{c}\text { Solution } \\
\text { time (in } \\
\text { seconds) }\end{array}$ & $\begin{array}{c}\text { Relative } \\
\text { Gap* }\end{array}$ & $\begin{array}{c}\text { Solution Time } \\
\text { Gap** }\end{array}$ \\
\hline 1 & 100 & 50 & 2,704 & 1.29 & $0.00 \%$ & $98.79 \%$ \\
2 & 100 & 50 & 3,381 & 7.12 & $0.00 \%$ & $98.79 \%$ \\
3 & 200 & 250 & 1,841 & 54.60 & $7.93 \%$ & $95.55 \%$ \\
4 & 250 & 1,000 & 2,601 & 112.18 & $15.68 \%$ & $59.22 \%$ \\
5 & 200 & 1,000 & 3,457 & 540.75 & $\mathbf{- 1 2 . 7 0 \%}$ & $\mathbf{- 1 8 , 8 4 8 . 7 2 \%}$ \\
\hline
\end{tabular}

* Relative gap is the percentage of the gap between the best known CPLEX ${ }^{\circledR}$ solution and the best GA solution. (Negative values show a better objective function value for the GA.)

** Solution time gap measures compare how long the CPLEX $^{\circledR}$ run lasts relative to the GA run. (Negative values show shorter solution times for the GA.)

*** Bold values indicate a better objective value or a shorter solution time for the GA.

The GA results suggest that for smaller problem instances, the MIP solutions can achieve optimal solutions in a shorter time. Yet, for larger problem instances, the GA can find better 
solutions than the MIP more quickly. The benefits of the GA are more significant with the cost minimization model where the number of nodes in the branch \& cut procedure of CPLEX ${ }^{\circledR}$ increases significantly. Although not shown on the tables, it should also be noted that the GA can find a feasible solution for any problem instance in less than one second, whereas the MIP procedure spends time on the order of ten minutes to find the first feasible solution for larger instances such as 4 and 5. Another important point is that the benefits of the GA diminish in the long term in the sense that after a certain amount of time, the GA reports fewer improvements where the MIP procedure would eventually find the optimal solution. These results are intuitive since after a near-optimal solution is found, it takes a longer time to find a better solution because of the random search aspects of the GA.

\section{Tabu Search}

In this research, the solution array of the problem is defined as a binary array, with each element corresponding to a component - supplier pair similar to the chromosome structure of the GA application. The move operator is selected to change one array element's value from 0 to 1 or vice versa on each iteration. As a result, the neighborhood is defined as the solutions that either have one more or one less active component - supplier pair. However, changing an array element from 1 to 0 , thus making this pair inactive, may lead to an infeasible solution. In this case, these infeasible solutions are determined and are not considered as candidate solutions for future moves. After a move is completed, the changed array element is added to the tabu list to prevent its use in future moves. However, even if a move is on the tabu list, solutions reachable through this move are still evaluated since an "aspiration criterion" is implemented. With this concept, if a tabu move leads to a solution with an objective value that is better than any known 
objective value so far, then this tabu move is allowed as an exception, thus preventing missing good opportunities in the solution space.

The TS algorithm keeps track of the best known solutions and when a solution better than the best one is not found for a certain number of iterations, it changes the current solution to the best known and clears the tabu list. With this long term memory application, branching towards worse regions of the solution space is stopped after some point and a fresh search is initiated from the best known point without any prevented moves by emptying the tabu list. If no improvement is noticed after a certain number of iterations (convergence) or a predetermined iteration limit is reached, the algorithm terminates.

Two main parameters of the TS, the tabu list size and the number of iterations limit, mainly determine the behavior of the algorithm. Therefore, these parameters are changed according to the size of the problem instance. All given values represent the starting tabu list size since the tabu list size is not fixed throughout the search. According to this policy, the tabu list size increased when a cycling between previous solutions occurs and it is reduced when no improvements are noted for a certain number of iterations. The number of iterations limit defines the maximum iterations allowed, however when the algorithm cannot improve the solution for a predetermined number of iterations, it is terminated. The preliminary TS application is able to minimize for both cost and lead time objectives. The pseudo-code of the TS implementation is given below. 


\section{TS Pseudo-code:}

Create initial solution

Until stopping criteria is met

Apply move to each solution array element and evaluate the objective value

If a tabu move has better objective than known so far apply aspiration, else set the non-tabu solution with best objective value as the new solution

Add changed solution array element to the tabu list

Update tabu list by removing oldest tabu move

If the best solution is not changed for a certain number of iterations then set best solution so far as current solution

Do

Check stopping criteria is met, if yes, then terminate; else, continue

For the five preliminary problem instances, Table 9 reports the results and the performance of the

TS algorithm for the cost minimization model. The lead time minimization model results and performance are shown in Table 10.

Table 9: Tabu Search cost minimization model preliminary run results

\begin{tabular}{cccrrrr}
\hline $\begin{array}{c}\text { Problem } \\
\text { Instance }\end{array}$ & $\begin{array}{c}\text { Tabu List } \\
\text { Size }\end{array}$ & $\begin{array}{c}\text { Number of } \\
\text { Iterations }\end{array}$ & $\begin{array}{c}\text { Best Objective } \\
\text { (\$) }\end{array}$ & $\begin{array}{c}\text { Solution } \\
\text { time (in } \\
\text { seconds) }\end{array}$ & $\begin{array}{c}\text { Relative } \\
\text { Gap* }\end{array}$ & \multicolumn{1}{c}{$\begin{array}{c}\text { Solution } \\
\text { Time Gap** }\end{array}$} \\
\hline 1 & 20 & 100 & $21,769,159$ & 0.19 & $0.00 \%$ & $42.11 \%$ \\
2 & 50 & 250 & $56,899,401$ & 22.93 & $0.00 \%$ & $99.08 \%$ \\
3 & 50 & 250 & $52,150,368$ & 434.17 & $10.01 \%$ & $\mathbf{- 4 6 6 . 0 8 \%} \%$ \\
4 & 50 & 250 & $133,883,711$ & $2,100.41$ & $39.43 \%$ & $\mathbf{- 2 3 , 4 3 0 . 9 4 \%} \%$ \\
5 & 50 & 250 & $689,614,166$ & $23,734.02$ & $44.67 \%$ & $\mathbf{- 3 7 9 . 2 2 \%} \%$ \\
\hline
\end{tabular}

* Relative gap is the percentage of the gap between the best known CPLEX $^{\circledR}$ solution and the best TS solution. (Negative values show a better objective function value for the TS.)

** Solution time gap measures compare how long the CPLEX ${ }^{\circledR}$ run lasts relative to the TS run (Negative values show shorter solution times for the TS).

*** Bold values indicate a better objective value or a shorter solution time for the TS. 
Table 10: Tabu Search lead time minimization model preliminary run results

\begin{tabular}{ccccccr}
\hline $\begin{array}{c}\text { Problem } \\
\text { Instance }\end{array}$ & $\begin{array}{c}\text { Tabu List } \\
\text { Size }\end{array}$ & $\begin{array}{c}\text { Number } \\
\text { of } \\
\text { Iterations }\end{array}$ & $\begin{array}{c}\text { Best } \\
\text { Objective } \\
\text { (hr) }\end{array}$ & $\begin{array}{c}\text { Solution } \\
\text { time (in } \\
\text { seconds) }\end{array}$ & $\begin{array}{c}\text { Relative } \\
\text { Gap* }\end{array}$ & $\begin{array}{c}\text { Solution } \\
\text { Time Gap** }\end{array}$ \\
\hline 1 & 20 & 100 & 2,704 & 0.17 & $0.00 \%$ & $23.53 \%$ \\
2 & 20 & 100 & 3,381 & 0.25 & $0.00 \%$ & $48.00 \%$ \\
3 & 20 & 500 & 1,706 & 27.04 & $0.64 \%$ & $79.33 \%$ \\
4 & 50 & 1,000 & 2,193 & 630.15 & $0.00 \%$ & $63.71 \%$ \\
5 & 50 & 200 & 4,717 & $1,144.32$ & $36.02 \%$ & $\mathbf{- 8 , 8 5 4 . 2 4 \%} \%$ \\
\hline
\end{tabular}

* Relative gap is the percentage of the gap between the best known CPLEX ${ }^{\circledR}$ solution and the best TS solution. (Negative values show a better objective function value for the TS.)

** Solution time gap measures compare how long the CPLEX $^{\circledR}$ run lasts relative to the TS run (Negative values show shorter solution times for the TS).

*** Bold values indicate a better objective value or a shorter solution time for the TS.

The TS lead time minimization results suggest that it performs very well for smaller instances and its performance is also good for the largest instance. However, for the middle-sized instances, it finds the optimal solution in a longer time compared to MIP solutions. This is a result of the defined neighborhood search policy in which the number of neighboring solutions increases extensively with the number of components and suppliers. However, due to the structure of the lead time problem, many neighboring solutions are degenerate, therefore the TS spends too much time searching different solutions with the same objective value. When compared to the GA, where the number of objective function calculations are fixed by the population size and degenerate solutions are very rare through crossover operations, the TS spends time on degenerate solutions and this causes worse computational time performance especially around a local optimal solution. In summary, GA outperforms TS as the problem size gets larger.

For the cost minimization problem, the TS performs well with the smaller instances, however with larger instances, solving the reduced cost minimization model for each candidate 
solution reduces the performance. It should also be noted that the number of neighboring solutions drastically increases with the larger problem instances. When compared to the GA, increasing the number of objective function calculations due to the problem size rather than having a fixed number of objective function calculations makes the GA preferable for larger problem instances. On the other hand, the TS outperforms the CPLEX ${ }^{\circledR}$ for the large instances by reaching good solutions in a shorter time although it takes longer to improve the solution for the TS than for the CPLEX ${ }^{\circledR}$. This suggests complex neighborhood and extensive objective function value evaluation procedures of the TS results in diminishing benefits during the search procedure resulting in a better performance than the MIP but a worse performance as compared to the GA.

These results suggest that none of the methods dominates another for all instances and each has certain time or solution quality advantages and disadvantages. However, in the long run, as the problem sizes gets larger, the GA starts to perform better compared to other methods.

\subsection{SELECTED SOLUTION METHODOLOGIES AND IMPLEMENTATION}

Based on the performance analyses of the different solution techniques on the preliminary models, the complete and reduced DFSC models are solved by using the MIP branch and cut method and the GA. The MIP guarantees finding the optimal solution and performs very good for smaller problem instances in particular. However, for larger problem instances, extensive search tree size creates a memory problem and finding the optimal solution takes very long. In

such cases, the GA is used to quickly find good, near-optimal solutions. The implementation details of both the MIP and the GA are given below. 


\subsubsection{Mixed Integer Programming}

As described in the preliminary modeling section (4.1.1), the MIP branch and cut method utilizes the CPLEX $^{\circledR}$ software. In order to solve MIP models, CPLEX ${ }^{\circledR}$ provides various options, including branching and node selection strategies, probing and cut generation alternatives, stopping criteria choices, etc. In order to select the best CPLEX ${ }^{\circledR}$ parameter set, an automated parameter tuning process, developed by Baz et al. [6], is utilized. In this process, a specifically designed program, Selection Tool for Optimization Parameters (STOP), works by solving a practically solvable size of the optimization model under different solver options in a structured way. First, a set of initial runs under different options were completed. Different solver options in these initial runs were selected by pairwise coverage; however STOP also allows the user to select these parameters randomly or by a greedy heuristic. Following that, a machine learning process based on regression trees (as artificial neural networks being another option) is employed to select optimization parameters for further runs. For CPLEX ${ }^{\circledR}$, the investigated optimization parameters consist of MIP emphasis (preference of concentrating on finding the optimal solution, a feasible solution, or their combination), node selection (strategy for selecting a node for exploration), branching variable selection (strategy for selecting a variable to branch on at each node), dive type (an option to look for the children nodes with probing before selecting the node), fractional cuts, and Mixed Integer Rounding (MIR) cuts. The results of 64 runs with various solver parameter combinations with problem instances with five to six components and suppliers did not show any significant solution time difference over the default solver parameters. The default CPLEX $^{\circledR}$ option for most of these parameters is for automatic selection and adjustment during the branch and cut procedure. The only two exceptions (that are not automatically adjusted during the branch and cut process) are the node selection strategy and the 
MIP emphasis parameters. However, changing these parameters from their default values did not have a significant impact on the solution time. In summary, all MIP computational tests are run by using the default CPLEX ${ }^{\circledR}$ parameters.

\subsubsection{Genetic Algorithm}

In order to solve the complete and reduced DFSC models, the GA progresses by deciding on two key problem variables, the price levels $\left(\right.$ price $_{t}$ ) and the component design alternative selections $\left(\pi_{i a_{i} t}\right)$ in each time period. Therefore, each GA chromosome has two main segments, one for

price and another for design selection variables. The complete GA chromosome structure is given in Figure 7. According to this structure, the price segment has $T$ genes, each corresponding to a pricing decision for a time period. These genes may get values changing from 1 to the number of pricing levels allowed by the problem instance. For example, if three price levels are considered for the models, then these genes may be valued as 1,2 , or 3 . It should be noted that, since there are no assumptions in the model limiting selection of price values for different time periods, any gene can take any of these allowed values independent of each other without causing any infeasibility.

In the component design selection segment of the chromosome, each gene corresponds to a selected - not selected decision for a component design alternative in a time period. Therefore, the genes are valued as 1 if the corresponding component design alternative is selected for the particular time period (i.e., $\pi_{i \alpha_{i} t}=1$ ) or as 0 if this component design alternative is not selected (i.e., $\pi_{i \alpha_{i} t}=0$ ). However, due to the constraint given in equation (6.9), these genes' values depend on each other for the representation of a feasible solution. Since only one design alternative can 
be selected for each component in each time period, only one of the genes may be 1 for a given i,t parameter value pair.

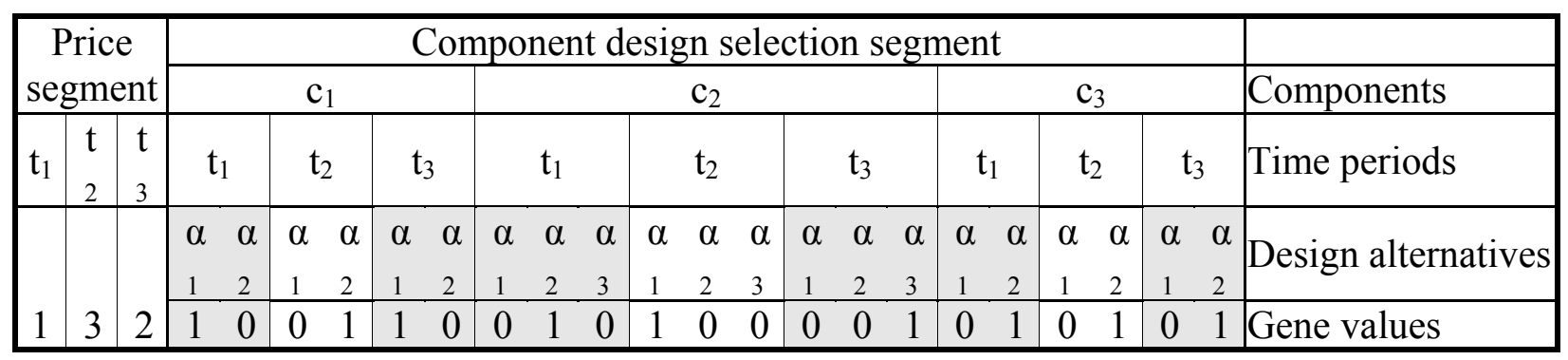

Figure 7: Chromosome structure of the Genetic Algorithm for complete and reduced models

The GA algorithm starts by creating the initial population. This is done uniform-randomly selecting a price value and component design alternatives for each time period. Since only one design alternative is selected for each component in each time period, initial chromosomes always correspond to feasible solutions.

Following the initial population, new generations are created by applying two major genetic operators and additional procedures. After copying a certain percentage of the best solutions to the next generation directly (elitist selections rule), new solutions are created by a crossover operator which selects two parents (two solutions from the previous generation) according to a roulette wheel selection rule (based on their fitness values) as described in the preliminary GA procedure. Then, for the crossover procedure, first two new offsprings are created. For the pricing decision in each time period, one of the two parents' gene values is selected with a higher probability ( 0.7 in this research) given to the parent with the better fitness value. These genes are then copied to the first offspring. The other offspring's price value for this time period is selected by the same procedure. After the price segment crossover is completed, 
the component design alternatives segment crossover is utilized. For each component, a parent (again the first parent with higher probability equal to 0.7 ) is selected for the first offspring and the other parent is assigned to the second offspring. Within each selected component, for each time period, again one of the parents is randomly selected by assigning a much higher probability (0.95 in this research) to the previously selected parent. In order to avoid a repair function as explained in section 4.2.2, all of the component alternatives' gene values are copied from the selected parent so that the crossover procedure always produces a feasible offspring. This crossover procedure mostly maintains each parent's gene structure via iteratively reducing the probability of selecting different parents from the component level to the time period level but selection of different parents for a component's different time periods is not completely eliminated.

After both offspring are formed with crossover, with a certain probability, these new chromosomes are mutated. The mutation is done separately for the pricing and component alternative selection segments. For the pricing segment, the price of a uniform-randomly selected time period is changed to another allowed price level with equal selection probabilities for other price levels. In the component design alternative segment, a component, and then a time period is uniform-randomly selected for mutation. Within this time period, the already selected component alternative design (which has a gene value of 1) is unselected by flipping the corresponding gene value to 0 . Among all other design alternatives, one is uniform-randomly picked and its gene value is flipped to 1 from 0 to select this design alternative. Therefore, this mutation procedure is ensured to keep feasibility of the offspring while providing some diversity to the GA procedure. The GA algorithm terminates either when a convergence is determined (no improvements are noted after a certain number of generations) or a time limit is reached. 
Within the GA procedure, each chromosome's fitness value (objective function value) is evaluated. Since the GA only picks the price and component design alternatives, the rest of the variables (production amounts, supplier selection, transportation flows, lead times, supply chain network, and economies of scale) need to be optimized for the given GA selections. Therefore, after each chromosome is completely produced (either by initial random selection, or by crossover and mutation), CPLEX $^{\circledR}$ is called to optimize other decisions via solving a smaller DFSC model, suited for the GA application. The GA model for the complete DFSC model is given below in equations (8.1) through (8.23) where corresponding GA models for the reduced DFSC models are listed in Appendix D. These GA models are based on their MIP counterparts and they follow the same notation and variables as well as same concepts and constraints. However, there are small modifications on these sub-models in order to reflect the GA decisions.

Since the GA algorithm selects price and component design alternatives, the complete DFSC is modified. Price,$\pi_{i \alpha i t}$ and Demand ${ }_{i t}$ variables of the complete DFSC model are still used in this GA sub-model but they are defined as parameters. Since two components of the demand function (price and product design) are selected by the GA, the demand values can be computed by the GA and inputted to the CPLEX ${ }^{\circledR}$ model. Due to the use of these variables as parameters, the linearization schemas are not employed in this model. Price - satisfied demand multiplication in the revenue function and demand - lead time multiplication in the inventory cost calculations do not introduce any nonlinearity for this model. Therefore, linearization related variables including $v_{t}, \phi_{1 t}, \phi_{2 t}, \lambda_{1 t}, \lambda_{2 t}, \psi_{1 t}, \psi_{2 t}, \psi_{3 t}, \psi_{4 t}, \delta_{n t}$, and $\gamma_{n t}$ are removed from the model.

The only newly introduced variable to this GA sub-model is LTint $_{t}$ which is used in inventory cost calculations instead of the $L T_{l t}$ variable as presented in the complete DFSC model. This addition ensures that the final lead time value used for inventory cost calculations is 
integer similar to the complete DFSC model lead time value for an equal comparison of both model results. The linearization of lead time and demand multiplication requires the final product's lead time $\left(L T_{l t}\right)$ to be an integer value and this integrality requirement is implied by other integer variables in the complete DFSC model. However, since the GA sub-models do not include these integer variables, this newly included lead time variable $\left(L\right.$ Tint $\left._{t}\right)$ ensures that the final product's lead time is an integer value and the GA results are comparable to the MIP results. Equation (8.13) is also introduced in order to establish the relationship between true lead time and its integer counterpart. Table 11 summarizes the asymptotic size analysis results for the GA sub-models.

Table 11: Summary of number of variables and constraints in GA sub-models

\begin{tabular}{lccc}
\hline $\begin{array}{l}\text { DFSC GA Sub- } \\
\text { Models }\end{array}$ & $\begin{array}{c}\text { Number of } \\
\text { Variables }\end{array}$ & $\begin{array}{c}\text { Number of } \\
\text { Integer } \\
\text { Variables }\end{array}$ & Number of Constraints \\
\hline $\begin{array}{l}\text { Complete DFSC GA } \\
\text { sub-model }\end{array}$ & $\mathrm{S}^{2}+$ & $1+\mathrm{S}^{2}+$ & \\
$\begin{array}{l}\text { Reduced DFSC GA } \\
\text { sub-model }-1\end{array}$ & $\mathrm{~T}\left(5+2 \mathrm{P}+\left[\left(\mathrm{PA}_{\mathrm{i}}\right)\left(3 \mathrm{~S}+\mathrm{S}^{2}\right)\right]\right.$ & $\mathrm{T}\left(2+\mathrm{PA}_{\mathrm{i}}[1+\mathrm{S}]\right)$ & $\mathrm{PT}\left[2+\mathrm{A}_{\mathrm{i}}\left(1+5 \mathrm{~S}+\mathrm{S}^{2}\right)+\mathrm{S}(1+\mathrm{P})\right]$ \\
$\begin{array}{l}\mathrm{S}^{2}+ \\
\text { Reduced DFSC GA } \\
\text { sub-model }-2\end{array}$ & $\left.\left.1+\mathrm{PA}_{\mathrm{i}}\right)\left(\mathrm{S}+\mathrm{S}^{2}\right)\right]$ & $\mathrm{T}\left(2+\mathrm{S}^{2}+\right.$ & $10 \mathrm{~T}+$ \\
\hline
\end{tabular}

The asymptotic size analysis shows that the GA sub-models have fewer variables and constraints than their counterpart MIP models as previously shown in Table 2. The number of available suppliers and the number of time periods have the most significant impact on the model size similar to the MIP models. Since the GA selects the product design and pricing decisions and therefore eliminates the associated variables, the impact of the number of components and design alternatives is reduced compared to the MIP models. Based on the previously used problem 
example (see section 3.5), with 10 components, 3 design alternatives for each component, 15 suppliers, 4 time periods, and 8 lead time - demand binary variables, Table 12 illustrates the number of variables and constraints in the GA sub-models.

Table 12: Numerical illustration of model asymptotic size of GA sub-models

\begin{tabular}{lccc}
\hline DFSC GA Sub-Models & $\begin{array}{c}\text { Number of } \\
\text { Variables }\end{array}$ & $\begin{array}{c}\text { Number of Integer } \\
\text { Variables }\end{array}$ & $\begin{array}{c}\text { Number of } \\
\text { Constraints }\end{array}$ \\
\hline $\begin{array}{l}\text { Complete DFSC GA } \\
\text { sub-model }\end{array}$ & 32,725 & 2,154 & 42,840 \\
$\begin{array}{l}\text { Reduced DFSC GA } \\
\text { sub-model - 1 }\end{array}$ & 29,125 & 354 & 39,240 \\
$\begin{array}{l}\text { Reduced DFSC GA } \\
\text { sub-model - } 2\end{array}$ & 32,641 & 2,153 & 40,400 \\
\hline
\end{tabular}

The GA illustration does not show a significant reduction either in the number of variables nor in the number of constraints compared to the MIP illustration (Table 3). The performance tests of the MIP and GA solution techniques (section 5.1) discuss as the significance of these reductions in number of variables and constraints and their impact on the solution performance. 


$$
\begin{aligned}
& \max \left(\sum_{t=1}^{T} \text { price }_{t}\left(k_{t}^{+}+k_{t}^{-}\right)\right)-\left(\sum_{t=1}^{T} \sum_{i=1}^{P} \sum_{j=1}^{S} \sum_{\alpha_{i}=1}^{A_{i}}\left(c_{i j \alpha_{i} t}^{1} x_{i j \alpha_{i} t}^{1}+c_{i j \alpha_{i} t}^{2} x_{i j \alpha_{i} t}^{2}\right)+\sum_{j=1}^{S} \sum_{l=1}^{S} y_{j l} \text { Netwco }_{j l}+\right. \\
& \left.\sum_{t=1}^{T} \sum_{j=1}^{S} \sum_{k=1}^{P} \sum_{l=1}^{S} \sum_{\alpha_{k}}^{A_{k}} u_{j k l \alpha_{k} t} \text { Tportcost }_{j k l \alpha_{k} t}+\sum_{t=1}^{T} \frac{1}{2} h_{t}\left(1+z_{\text {ssratio }} \rho_{2}\right) \text { Demand }_{1 t} \text { LTint }_{1 t}\right)
\end{aligned}
$$

s.t.

$$
\begin{array}{ll}
\sum_{\alpha_{k}=1}^{A_{k}} \sum_{j=1}^{S}\left(x_{k j \alpha_{k} t}^{1}+x_{k j \alpha_{k} t}^{2}\right) \geq \sum_{i=1}^{k-1} \sum_{\alpha_{i}=1}^{A_{i}} \sum_{j=1}^{S}\left(x_{i j \alpha_{i} t}^{1}+x_{i j \alpha_{i} t}^{2}\right)_{\text {Relation }_{i k}} \quad \forall k(k>1), t \\
\sum_{j=1}^{S} x_{i j \alpha_{i} t}^{1} \leq \pi_{i \alpha_{i} t} \text { Mcap }_{i \alpha_{i} t} & \forall i, \alpha_{i}, t \\
x_{i j \alpha_{i} t}^{1} \leq \text { Capacity }_{i j \alpha_{i} t}^{1} & \forall i, j, \alpha_{i}, t \\
x_{i j \alpha_{i} t}^{1} \geq \text { Capacity }_{i j \alpha_{i} t}^{1} a_{i j \alpha_{i} t} & \forall i, j, \alpha_{i}, t \\
x_{i j \alpha_{i} t}^{2} \leq \text { Capacity }_{i j \alpha_{i} t}^{2} a_{i j \alpha_{i} t} & \forall i, j, \alpha_{i}, t \\
y_{j l}\left(\text { Capacity }_{k l \alpha_{k} t}^{1}+\text { Capacity }_{k l \alpha_{k} t}^{2}\right) \geq u_{j k l \alpha_{k} t} & \forall j, k, l, \alpha_{k}, t \\
\sum_{j=1}^{S} u_{j k l \alpha_{k} t} \leq x_{k l \alpha_{k} t}^{1}+x_{k l \alpha_{k} t}^{2} & \forall k, l, \alpha_{k}, t \\
\sum_{\alpha_{k}=1}^{A_{k}} \sum_{l=1}^{S} u_{j k l \alpha_{k} t} \geq \text { Relation }_{i k} \sum_{\alpha_{i}=1}^{A_{i}}\left(x_{i j \alpha_{i} t}^{1}+x_{i j \alpha_{i} t}^{2}\right) & \forall i, j, k, t \mid \text { Relation }_{i k}>0
\end{array}
$$

ptime $_{i t} \geq p t_{i j \alpha_{i} t}\left(\frac{x_{i j \alpha_{i} t}^{1}}{\text { Capacity }_{i j \alpha_{i} t}^{1}}+\frac{x_{i j \alpha_{i} t}^{2}}{\text { Capacity }_{i j \alpha_{i} t}^{2}}\right) \quad \forall i, j, \alpha_{i}, t \mid$ Capacity $_{i j \alpha t}^{1}>0$

$L T_{i t} \geq L T_{k t}$ Relation $_{i k}+$ ptime $_{i t}$

$\forall i, k, t \mid$ Relation $_{i k}>0$

$L T_{i t} \geq$ ptime $_{i t}$

$\forall i, t \mid$ Relation $_{i k}=0$ for $\forall k>i$

Lint $_{t} \geq L T_{1 t}$

$\forall t$

$\tau_{t}^{+} \sum_{\alpha_{1}=1}^{A_{1}} \operatorname{Mcap}_{1 \alpha_{1} t} \geq \sum_{\alpha_{1}=1}^{A_{1}} \sum_{j=1}^{S}\left(x_{1 j \alpha_{1} t}^{1}+x_{1 j \alpha_{1} t}^{2}\right)-$ Demand $_{1 t} \quad \forall t$

$\tau_{t}^{-}$Mdem $\geq$Demand $_{1 t}-\sum_{\alpha_{1}=1}^{A_{1}} \sum_{j=1}^{S}\left(x_{1 j \alpha_{1} t}^{1}+x_{1 j \alpha_{1} t}^{2}\right)$

$\tau_{t}^{+}+\tau_{t}^{-} \leq 1$

$\forall t$

$k_{t}^{+} \leq$Demand $_{1 t}$

$k_{t}^{+} \leq \tau_{t}^{+}$Mdem

Demand $_{1 t}+\tau_{t}^{+}$Mdem $\leq k_{t}^{+}+$Mdem

$k_{t}^{-} \leq \sum_{\alpha_{1}=1}^{A_{1}} \sum_{j=1}^{S}\left(x_{1 j \alpha_{1} t}^{1}+x_{1 j \alpha_{1} t}^{2}\right)$

$k_{t}^{-} \leq \tau_{t}^{-} \sum_{\alpha_{1}=1}^{A_{1}} \operatorname{Mcap}_{1 \alpha_{1} t}$

$\sum_{\alpha_{1}=1}^{A_{1}} \sum_{j=1}^{S}\left(x_{1 j \alpha_{1} t}^{1}+x_{1 j \alpha_{1} t}^{2}\right)+\tau_{t}^{-} \sum_{\alpha_{1}=1}^{A_{1}} \operatorname{Mcap}_{1 \alpha_{1} t} \leq k_{t}^{-}+\sum_{\alpha_{1}=1}^{A_{1}} \operatorname{Mcap}_{1 \alpha_{1} t} \quad \forall t$

$x_{i j \alpha_{i} t}^{1}, x_{i j \alpha_{i} t}^{2}, u_{j k l \alpha_{k} t}, L T_{i t}$, ptime $_{i t}, k_{t}^{+}, k_{t}^{-}$, Demand $_{k t} \geq 0 ;$ LTint $_{t} \geq 0$ \& integer;

$\pi_{i \alpha_{i} t}, y_{j l}, a_{i j \alpha_{i} t}, \tau_{t}^{+}, \tau_{t}^{-}, \in\{0,1\}$ 
The complete pseudo-code of the GA implementation is given below.

\section{Complete GA Pseudo-code:}

\section{Create initial population \\ Until stopping criteria is met \\ Copy elitist chromosomes to next generation \\ Until population size is filled}

Select two parents according to the roulette wheel rule

For each offspring

For each time period

Select one of the parents (higher probability given to the parent with better fitness value)

Copy the corresponding gene value

Next time period

Next offspring

For each offspring

For each component

Select a parent (with higher probability given to the first parent)

For each time period

Select a parent (higher probability given to the assigned parent)

Copy gene values for this component and time period

Next time period

Next component

Next offspring

Decide to mutate or not

If mutate

Randomly perturb the pricing for a time period

Randomly perturb a component design alternative selection End if

Do

Calculate fitness values of the chromosomes

Do

Check stopping criteria is met, if yes, then terminate; else, continue

Since the GA is a random search algorithm, different parameters impact the solution time and quality and different runs may produce different results. A parameter tuning test based on the preliminary models is explained in section 4.2.2. The rule of thumb suggests that increasing population size and number of generations would yield better solutions (with the expense of longer solution times) since more of the solution space will be searched. The preliminary analyses also support this outcome. On the other hand, increasing mutation probability and 
percentage of elitist chromosomes have different impacts on the solution quality and time. A higher mutation rate endangers the survival of the good chromosomes (or chromosome segments) by randomly altering the genes too frequently. However, a low mutation rate would not help to diversify the population since the crossover operation always picks the genes from the existing gene structures. Therefore, for a balanced diversification and preservation of the population, a dynamic mutation rate is used [50]. According to this, the GA starts with a low mutation rate since the algorithm is more likely to improve the best-known solution during the early generations. However, as the best-known solution improves and the search progresses to later generations, it takes longer to find a better solution due to the random nature of the algorithm. In these phases, a larger mutation rate helps to diversify the population by random perturbation of the chromosomes [11].

In addition to the mutation, survival of the elitist chromosome helps to exploit the best-known solutions to find better solutions within the neighborhood. According to this concept, a predetermined percentage of the best chromosomes of the population survives (copied unchanged) to the next generation based on their fitness values. This procedure ensures that a population always contains the best-known solutions and it survives until a better one is found. Since, according to the roulette wheel selection rule, a chromosome with a better fitness value is more likely to be selected for crossover, the elitist approach also guides the algorithm to make more use of the best-known solutions. However, a too high elitist percentage risks the diversity of the population by filling the new generation with too many of the chromosomes from the previous generations. A too low percentage would lead to loss of good chromosomes and thus the algorithm cannot exploit the good chromosomes to the possible extent. Therefore, the percentage of the elitist chromosomes for larger populations is increased. According to this 
approach, a small population size would keep only a small percentage of the elitist chromosomes where more of these good solutions can be kept and exploited when larger populations are maintained.

\subsection{SOLUTION TECHNIQUES COMPLEXITY ANALYSIS}

\section{Preliminary Model Solution Techniques}

Most of the MIP models, especially which do not satisfy specific conditions (e.g., with integer right hand side values or with a totally unimodular constraint matrix) tend to be NP-hard. In addition, most of the supply chain problems are modeled as MIP models. Although the complexity of the developed DFSC models is not explicitly proven, like most non-trivial supply chain models, it is expected that these models are NP-hard. The performance of the solution techniques discussed in sections 4.1.2 and 4.2.2 suggest that it is significantly harder to solve the developed DFSC models as these models grow in size and they do not guarantee a polynomial

time solution. In addition, for the CPLEX ${ }^{\circledR}$ MIP solver, the complexity of the solution algorithm depends on the relaxed linear programming solution (with the fractional values of integer variables) and the relationships between the components and suppliers.

Both of the heuristic algorithms evaluated in preliminary modeling (GA and TS) start with creating feasible solutions by fixing variables. This process takes $O(P S)$ computational time where $O$ denotes an asymptotic upper bound for the time of the process. It should be noted that in the GA, the number of chromosomes is equal to the population size for a single generation (thus the process takes $O($ pop_size $\times P S)$ ) where the TS generates only a single solution at each 
iteration. After the initial solution set is created, the GA uses three operations to create the next generation:

1) copying elitist chromosomes $(O(\#$ elitist $\times P S))$,

2) creating immigrating chromosomes $(O(\#$ immigrating $\times P S))$, and

3) crossover and mutation $(O(\#$ crossover $\times P S))$.

Therefore, each generation of the GA lasts $O($ pop_size $\times P S)$, and for a given number of generations, the GA takes $O(\# g$ enerations $\times$ pop_size $\times P S)$ time.

On the other hand, the TS investigates each neighboring solution of the current one by flipping a single bit every time. Thus the search takes $O(P S)$ computational time. The move operation picks the best flip and moves to this solution which takes $O(1)$ time since only a single bit is changed in each move. As a result, for a given iteration limit, the TS algorithm takes $O(\#$ iterations $\times P S)$ time. However, since both algorithms employ a CPLEX ${ }^{\circledR}$ MIP solution procedure to calculate the objective function by using branch and cut and simplex algorithms, neither algorithm is guaranteed to run in polynomial time suggesting a significant increase in solution times as the problem sizes increases.

\section{Complete and Reduced Model Solution Techniques}

Similar to the preliminary models, the complete and reduced DFSC model solution obtained by using the branch and cut technique depends on the interactions between components and suppliers (thus on the problem instance) and the problem size. Since the branch and bound tree would grow as the problem size gets larger, it increases the solution time. However, the growth of the solution tree size cannot be analytically determined since it depends on the initial relaxation results, the number of integer variables, and the problem instance itself. The model complexity analysis proposes that the number of binary variables is $S^{2}+T\left(4+N+\left[\left(P A_{i}\right)(1+S)\right]\right)$ in 
the complete DFSC model therefore an upper bound on the size of the branch and bound solution tree would be $2^{S^{2}+T(4+N+[(P A i)(1+S)])}$.

The GA solution procedure starts with creating an initial population which selects component design alternatives and prices for each time period. This process takes $O\left(P A_{i} T\right)$ time therefore it is polynomial. For the creation of the following generations, the elitist chromosome copying and crossover procedures take $\mathrm{O}\left(p o p \_s i z e \times P A_{i} T\right)$ time in total, since each of these procedures requires copying each gene to a new offspring. However, the number of iterations (generations) the GA will use is not known since it depends on the improvements from one generation to the next. In addition, each chromosome's fitness value is calculated by another branch and cut algorithm to solve for the remaining variables. In conclusion, since the number of generations changes randomly for each GA run and the branch and cut algorithm is not polynomial, the GA eventually cannot run in polynomial time. 


\subsection{COMPUTATIONAL RESULTS}

In this section, the computational results are presented. The first section reports on the performance of the MIP and GA solution techniques on the complete and reduced DFSC models. The solution quality and solution time are reported with a comparative analysis. The following section concentrates on the research questions stated in section 1.3. Each research question is investigated and the behavior of the models, solution techniques, and results are discussed. The third section summarizes the validity of the models and the results via academic literature survey and industry experts' views.

\subsection{PERFORMANCE TESTS ON COMPLETE AND REDUCED MODELS}

The performance of the MIP and GA solution techniques are discussed in this section. In order to evaluate their performances, five complete DFSC model instances are created randomly. Table 13 shows the number of components and available suppliers used in these problem instances. Similar to the preliminary modeling, these instances grow in size to evaluate the performance of the solution methods for different problem sizes. 
Table 13: Complete and reduced DFSC problem instances for performance testing

\begin{tabular}{ccc}
\hline Problem Instance & Number of Components & Number of all Suppliers \\
\hline 1 & 4 & 6 \\
2 & 6 & 9 \\
3 & 8 & 12 \\
4 & 10 & 15 \\
5 & 16 & 24 \\
\hline
\end{tabular}

For all five instances, four time periods are considered. Each time period in the model corresponds to a product life cycle phase, namely introduction, growth, maturity, and decline as suggested by Perreault and McCarthy [49].

In these problem instances, the number of design alternatives for each component is uniform-randomly selected between 1 and 3. For the Bill of Materials relationships, a uniform-random assignment is followed where any number of a component between zero and five may be required by another component. It should be noted that if zero units of a component are required by another component, it is not used. The production time for each component is selected between 1 and 30 days uniform-randomly. By performing a presolve analysis for each instance, the maximum lead time is determined to be less than 256 days $\left(2^{8}\right.$ days) for each instance. This bound necessitates eight binary lead time linearization variables $\left(\gamma_{n t}\right)$ to be used for these problem instances.

\subsubsection{Mixed Integer Programming}

For the performance testing of the branch and cut method, CPLEX ${ }^{\circledR}$ is utilized with the default parameters as suggested by the STOP procedure described in section 4.3.1. For each problem 
instance, the branch and cut algorithm is allowed to run for 48 hours $(172,800$ seconds) or until the optimal solution is found (by a $0.01 \%$ optimality gap). The optimality gap measures the relative ratio of the objective value of the best integer feasible solution found to the objective value of the best possible solution (a bound) which is the best objective value of a node in the MIP solution tree. When the optimality gap value is equal to zero, the obtained solution is guaranteed to be the optimal one. Larger optimality gap values mean the true optimal solution is between the solution obtained and the bound. For any optimality gap value other than zero, the current solution may be the optimal solution but it is not guaranteed nor proven. Table 14 reports the results of the MIP runs with the complete DFSC model instances.

Table 14: MIP results for the complete DFSC model

\begin{tabular}{ccc}
\hline Problem Instance & Optimality Gap & Solution Time (sec.) \\
\hline 1 & $0.01 \%$ & 150.69 \\
2 & $20.61 \%$ & $172,800.00$ \\
3 & $2,490.00 \%$ & $172,800.00$ \\
4 & $92.01 \%$ & $172,800.00$ \\
5 & $96.03 \%$ & $172,800.00$ \\
\hline
\end{tabular}

The results show that although a feasible solution to the problem is found for every problem instance, they cannot be solved to optimality with the given 48 hour time limit (except the smallest instance). Another important result is that the solution quality depends not only on the problem size but also on the problem instance as well. For example, the two largest problems (4 and 5) can be solved to around $90 \%$ optimality, but the third instance result is only within the $2,490 \%$ of the optimality bound. Therefore, the MIP solution progress cannot be easily determined by only assessing the problem size. Moreover, the results show that the complete DFSC is very complex and hard to solve even for small scale problems. 
In order to further evaluate the performance of the MIP algorithm, the reduced DFSC model -1 is solved by using the same problem instances and with the same 48 hour time limit. Table 15 summarizes the results.

Table 15: MIP results for the reduced DFSC model - 1

\begin{tabular}{ccc}
\hline Problem Instance & Optimality Gap & Solution Time (sec.) \\
\hline 1 & $0.01 \%$ & 36.89 \\
2 & $0.01 \%$ & $48,091.53$ \\
3 & $15.64 \%$ & $172,800.00$ \\
4 & $36.60 \%$ & $172,800.00$ \\
5 & $33.11 \%$ & $172,800.00$ \\
\hline
\end{tabular}

The results show that the solution quality is increased for all instances compared to the complete model. As suggested by the model complexity analysis, having fewer binary variables helps the algorithm progress faster. These results still suggest that the solution quality degrades as the problems get larger and solution time increases. However, compared to the complete model, the algorithm finds better solutions in the same running time or even in a shorter time as seen in problem instance 2 .

In order to evaluate the performance of the MIP solution technique further, the reduced DFSC model -2 is solved by using the same problem instances. The results are shown in Table 16.

Table 16: MIP results for the reduced DFSC model - 2

\begin{tabular}{ccc}
\hline Problem Instance & Optimality Gap & Solution Time (sec.) \\
\hline 1 & $0.01 \%$ & 6.24 \\
2 & $0.01 \%$ & $7,023.81$ \\
3 & $0.01 \%$ & $145,232.95$ \\
4 & $49.63 \%$ & $172,800.00$ \\
5 & $16.34 \%$ & $172,800.00$ \\
\hline
\end{tabular}


The reduced DFSC model - 2 MIP results show that, in general, the performance increase is even better than the one for the reduced DFSC model -1 . Three of the five problem instances could be solved to optimality and the largest problem is solved to less than a $17 \%$ optimality gap, best among all three tests. However, the solution quality for the fourth instance is worse than that of for the reduced DFSC model -1 . These results suggest that the performance of the MIP solution algorithm is not necessarily correlated with the number of binary variables in the model as the reduced DFSC model -1 has fewer binary variables than the reduced DFSC model -2 . The interactions between these variables are very important for faster reduction of the optimality bound. However, in general, it is clear that the algorithm performance deteriorates as the problems get larger.

\subsubsection{Genetic Algorithms}

In order to test the performance of the GA, the described five problem instances are solved for both the complete and the reduced DFSC models. The GA procedure is allowed to run for 48 hours (the same limit applied to the MIP) or it is terminated when no improvements are noted in 20 consecutive generations. Initial test runs without the objective improvement termination condition showed that once no improvements are noted for about 10 iterations, the GA often cannot find a better solution. Therefore, setting the termination limit to 20 consecutive generations without any improvements is reasonable. Each GA run is started with a 5\% elitist chromosome rate and a $1 \%$ mutation rate. However, according to the dynamic mutation rate application, the mutation rate is increased by $1 \%$ when no improvements in the best-known solution are noted for three consecutive generations. The solution quality (optimality gap) of the GA is measured relatively to the final solution of the MIP technique. For example, if the MIP 
finds the optimal solution and the GA does not, then there is an optimality gap for the GA solution. The negative optimality gap values mean that the GA solution is worse than the MIP solution (since the objective is to maximize the profit) where the positive values correspond to the GA solutions which are better than the MIP solutions. Since the MIP guarantees an optimal solution (if one exists), the GA can only find better solutions when the MIP runs are stopped due to meeting the time limit before the optimal solution is found. The solution time gap values on the other hand are different. The negative solution time gaps correspond to faster GA runs where positive solution time gap values mean that it took the GA longer to achieve the reported results. Table 17 summarizes the results of the GA with the complete DFSC model.

Table 17: GA results for the complete DFSC model

\begin{tabular}{cccc}
\hline Problem Instance & Objective Value Gap & Solution Time Gap & Solution Time (sec.) \\
\hline 1 & $0.00 \%$ & $99.56 \%$ & 300.72 \\
2 & $-0.04 \%$ & $\mathbf{- 9 9 . 8 3 \%}$ & 291.40 \\
3 & $\mathbf{5 . 3 0 \%}$ & $\mathbf{- 7 4 . 3 3 \%}$ & $44,354.28$ \\
4 & $\mathbf{6 . 6 6 \%}$ & $\mathbf{- 9 8 . 5 4 \%}$ & $2,529.32$ \\
5 & $-18.53 \%$ & $\mathbf{- 3 8 . 1 7 \%}$ & $106,841.33$ \\
\hline
\end{tabular}

Bold values indicate a better objective value or a shorter solution time for the GA.

According to the results, the GA usually terminates before the MIP, except for the smallest instance due to the requirement of searching for an extra 20 generations with no improvements. Since the GA does not use any optimality bound information, these additional generations increase the solution time even if the optimal solution is found as shown with the first problem instance. Due to the random nature of the GA, a solution quality bound cannot be given. However, results show that the GA finds the optimal solution for the smallest instance and it finds better solutions than the MIP for the third and fourth problem instances. For the second and the fifth (largest) problem instances, it cannot find a better solution than the MIP, suggesting a 
highly random solution quality. However, the GA usually runs for shorter time periods compared to the MIP and the solution quality is always less than $20 \%$ worse compared to the MIP.

Table 18 reports the results of the GA application for the reduced DFSC model - 1 where the economies of scale concept is removed.

Table 18: GA results for the reduced DFSC model - 1

\begin{tabular}{cccc}
\hline Problem Instance & Objective Value Gap & Solution Time Gap & Solution Time (sec.) \\
\hline 1 & $0.00 \%$ & $75.52 \%$ & 64.75 \\
2 & $0.00 \%$ & $\mathbf{- 9 9 . 8 7 \%}$ & 60.13 \\
3 & $\mathbf{5 . 7 2 \%}$ & $\mathbf{- 9 8 . 0 3 \%}$ & $3,396.70$ \\
4 & $\mathbf{4 . 0 0 \%}$ & $\mathbf{- 9 8 . 5 7 \%}$ & $2,465.88$ \\
5 & $-9.87 \%$ & $0.00 \%$ & $172,800.00$ \\
\hline
\end{tabular}

"Bold values indicate a better objective value or a shorter solution time for the GA.

The reduced DFSC model - 1 GA run results show that the GA performs better with the reduced model as it finds the optimal solution for the first and second instances and finds better solutions than the MIP for the third and fourth problem instances. For the largest instance, the solution quality is less than $10 \%$ of the MIP where both algorithms terminate due to the time limit. However, the GA runs are often shorter than the MIP runs except for the smallest instance where the additional 20 generations requirement for termination adds extra time to the algorithm and becomes significant. As the last performance test for the GA, results of the reduced DFSC model -2 are summarized in Table 19. 
Table 19: GA results for the reduced DFSC model -2

\begin{tabular}{cccc}
\hline Problem Instance & Objective Value Gap & Solution Time Gap & Solution Time (sec.) \\
\hline 1 & $0.00 \%$ & $1,734.18 \%$ & 114.45 \\
2 & $0.00 \%$ & $\mathbf{- 9 8 . 2 6 \%}$ & 122.39 \\
3 & $-0.12 \%$ & $\mathbf{- 7 0 . 6 6 \%}$ & $42,608.76$ \\
4 & $-4.64 \%$ & $\mathbf{- 8 6 . 2 5 \%}$ & $23,767.98$ \\
5 & $-4.73 \%$ & $\mathbf{- 6 6 . 8 7 \%}$ & $57,246.45$ \\
\hline
\end{tabular}

Bold values indicate a shorter solution time for the GA.

The reduced DFSC model -2 results show that the GA can find very good solutions (less than $5 \%$ worse than the MIP) in a relatively short time, except for the smallest instance due to the termination requirement described before. In the worst case, for the largest problem instance, the GA runs for nearly 16 hours where the MIP runs up to the 48 hour time limit.

\subsubsection{Comparative Analysis}

The final run results for the MIP and GA solution techniques only show the outcomes of the individual runs within the allowed time limits. However, the progression of both solution techniques are important to accurately compare their performances. For example, since the MIP procedure tries to find the true optimal solution to the problem, it continues to run even after the optimal solution is found to prove its optimality. On the other hand, the GA procedure terminates regardless of the goodness of the solution and does not try to prove optimality or to provide any optimality gap information. Therefore, the actual optimization process data is illustrated to compare the behavior of each solution technique over the run-time.

For each of the problem instances, six different runs (MIP and GA runs for the complete model and the reduced models 1 and 2) are illustrated on the following charts. The $\mathrm{x}$-axis shows the run time in a logarithmic scale where the y-axis shows the actual solution value. Since both 
algorithms find a feasible solution with a positive objective value very quickly (in less than $0.05 \%$ of their total run times), the y-axis on the graphs only shows positive objective values for clarity. The progress of both algorithms for the first problem instance is shown in Figure 8 for the complete DFSC model, in Figure 9 for the reduced DFSC model - 1, and in Figure 10 for the reduced DFSC model -2 .

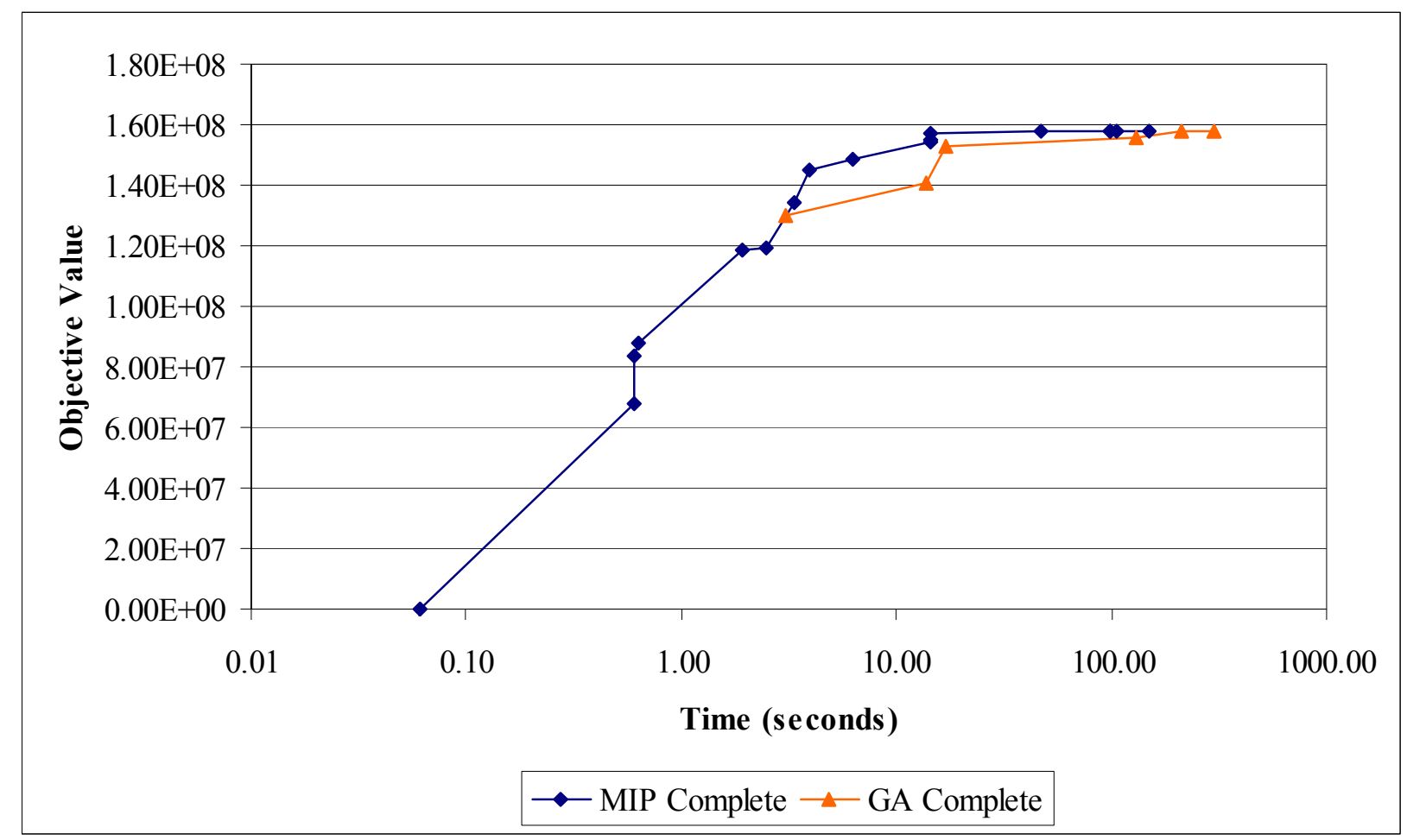

Figure 8: Progress of the MIP and the GA for the complete DFSC model (instance 1) 


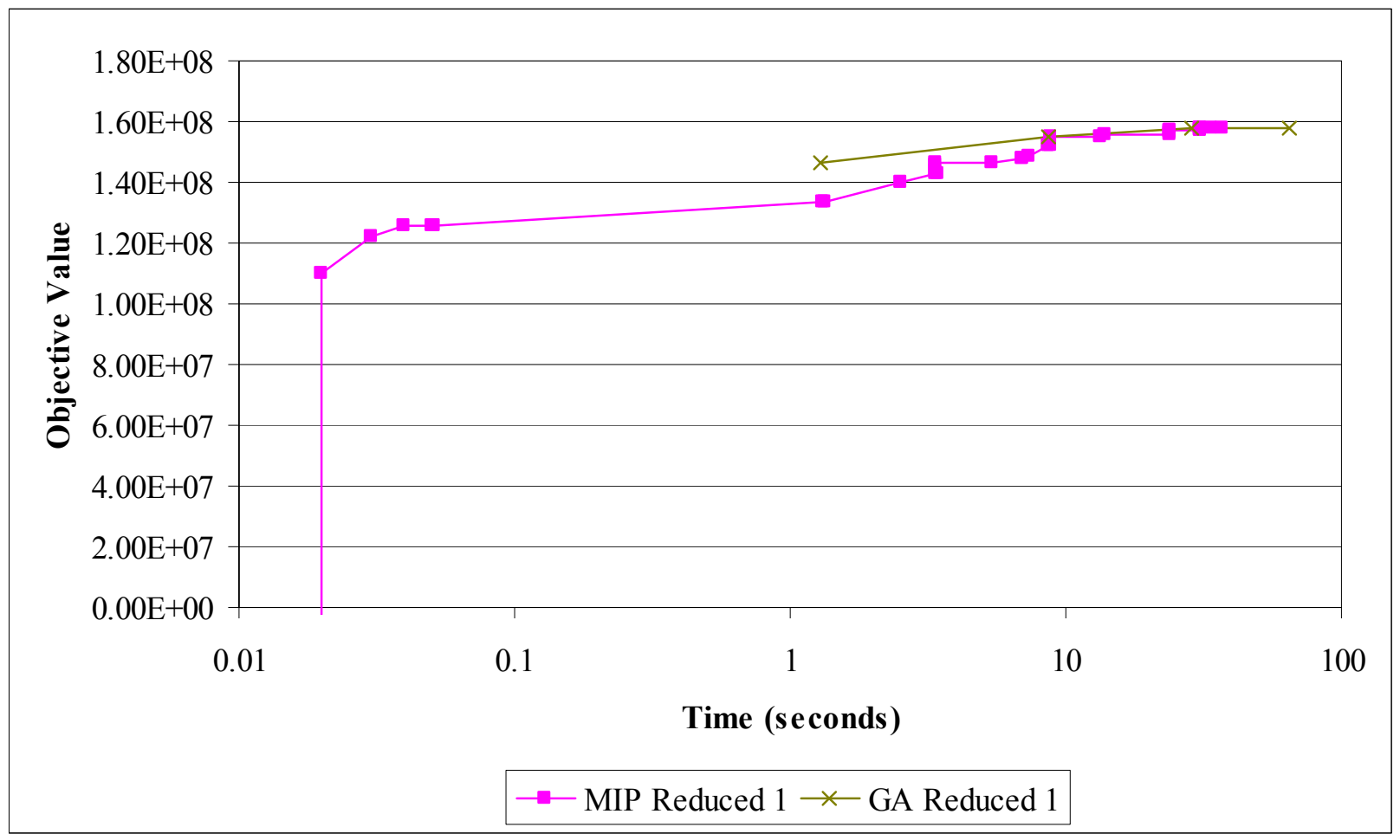

Figure 9: Progress of the MIP and the GA for the reduced DFSC model - 1 (instance 1)

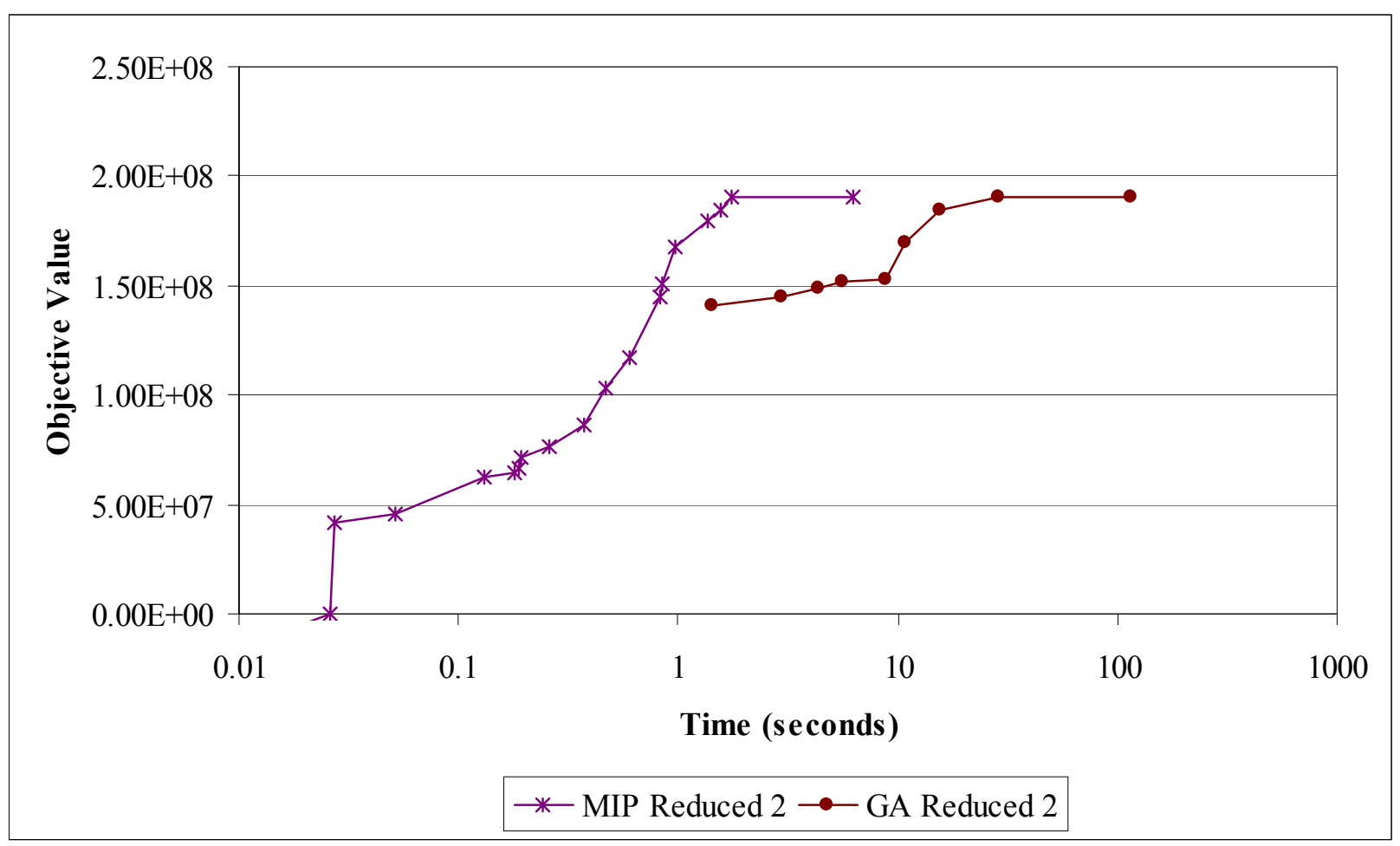

Figure 10: Progress of the MIP and the GA for the reduced DFSC model - 2 (instance 1) 
The algorithm progressions show that for this smallest problem instance, the MIP solves the problem to the optimality for all models in a shorter time than the GA. The MIP dominates the GA for the complete DFSC model and the reduced DFSC model -2. Although the GA finds a better solution than the MIP initially for the reduced DFSC model - 1, the MIP outperforms the GA by finding the optimal solution in a shorter time. The progression of both algorithms for the second problem instance is shown in Figure 11 for the complete DFSC model, in Figure 12 for the reduced DFSC model - 1, and in Figure 13 for the reduced DFSC model -2 .

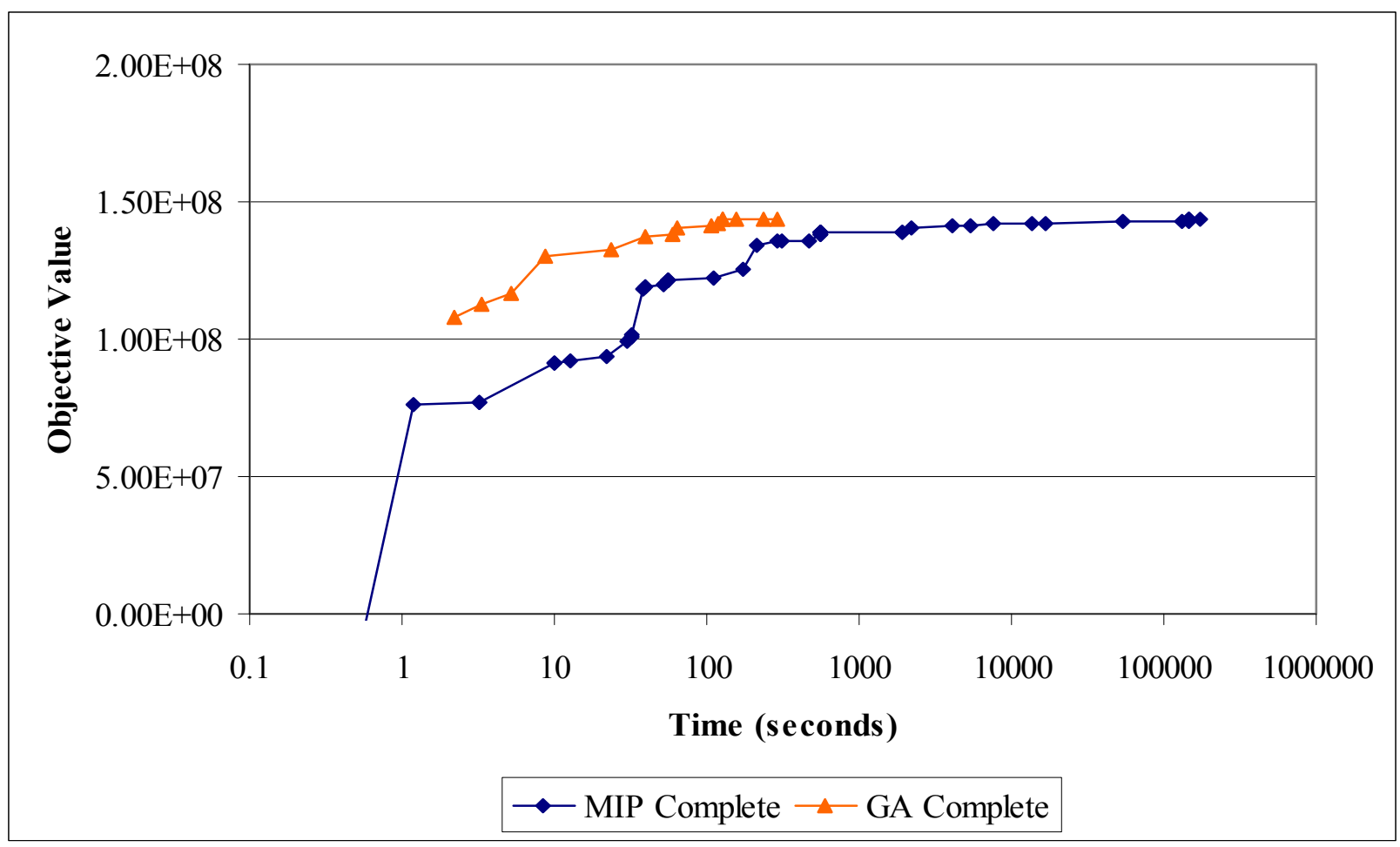

Figure 11: Progress of the MIP and the GA for the complete DFSC model (instance 2) 


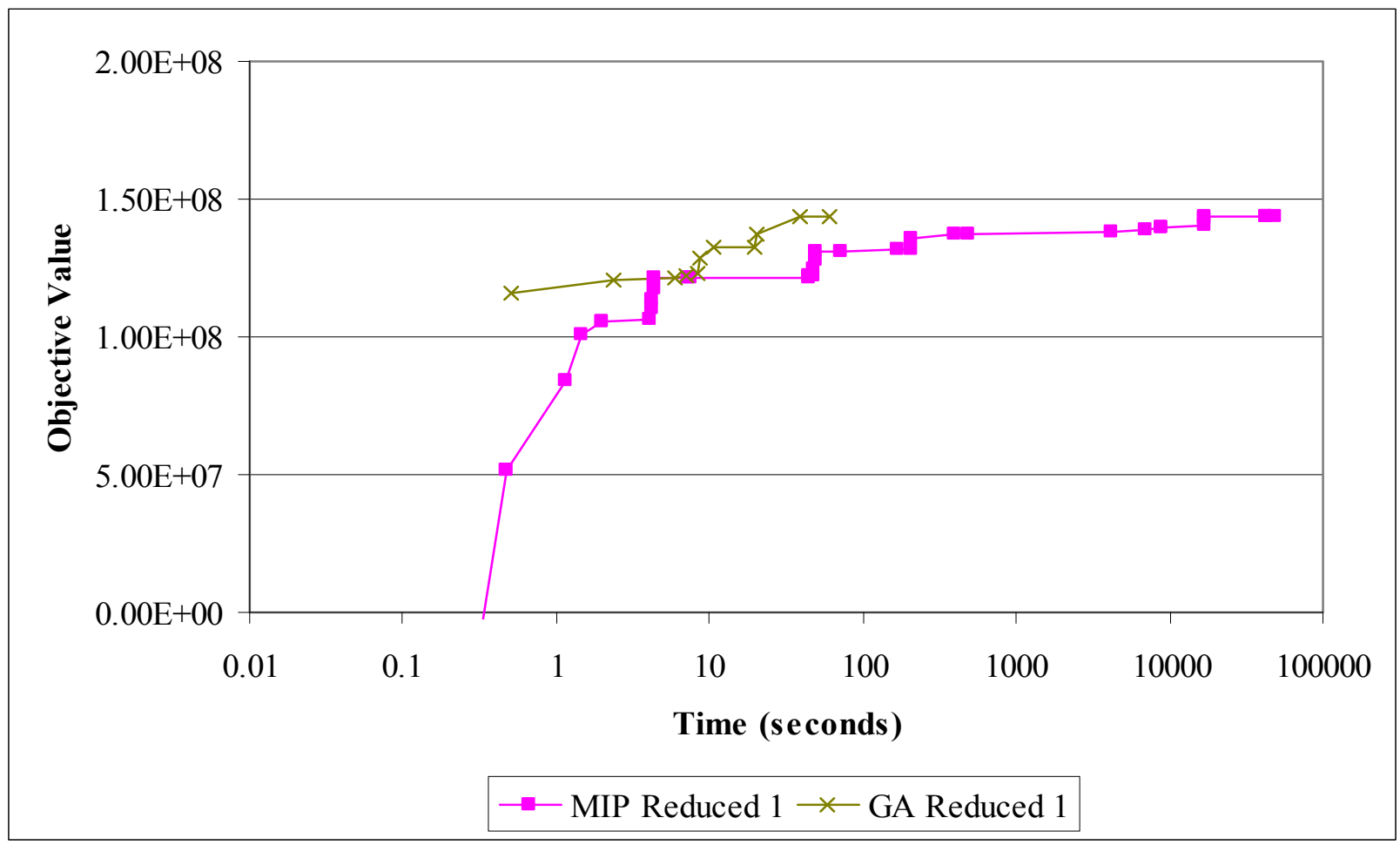

Figure 12: Progress of the MIP and the GA for the reduced DFSC model - 1 (instance 2)

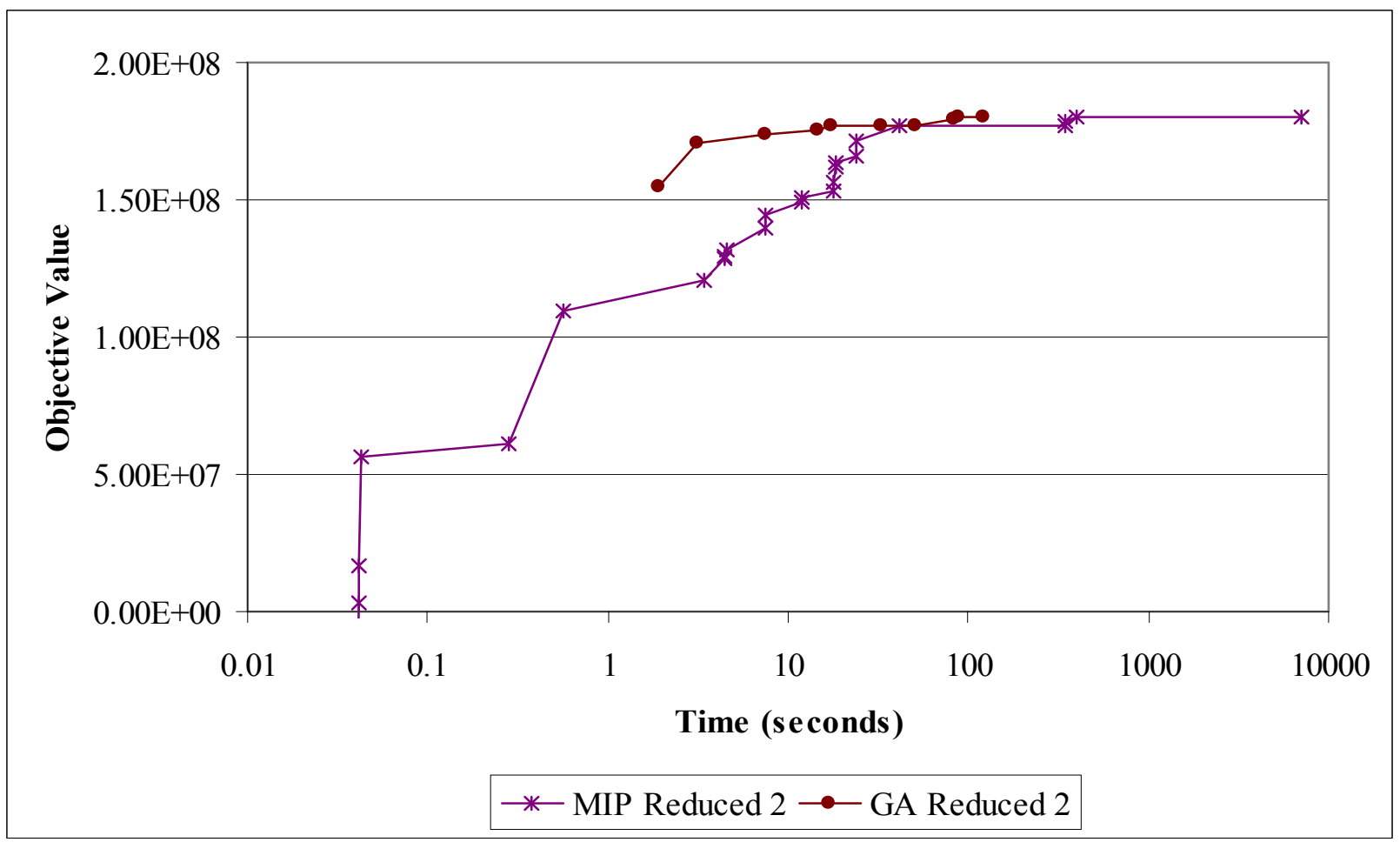

Figure 13: Progress of the MIP and the GA for the reduced DFSC model - 2 (instance 2) 
For the second problem instance, as opposed to the first instance, the GA dominates the MIP for all three models by finding the solutions with better objective values in a shorter time. The progress of both algorithms for the third problem instance is shown in Figure 14 for the complete DFSC model, in Figure 15 for the reduced DFSC model - 1, and in Figure 16 for the reduced DFSC model -2 .

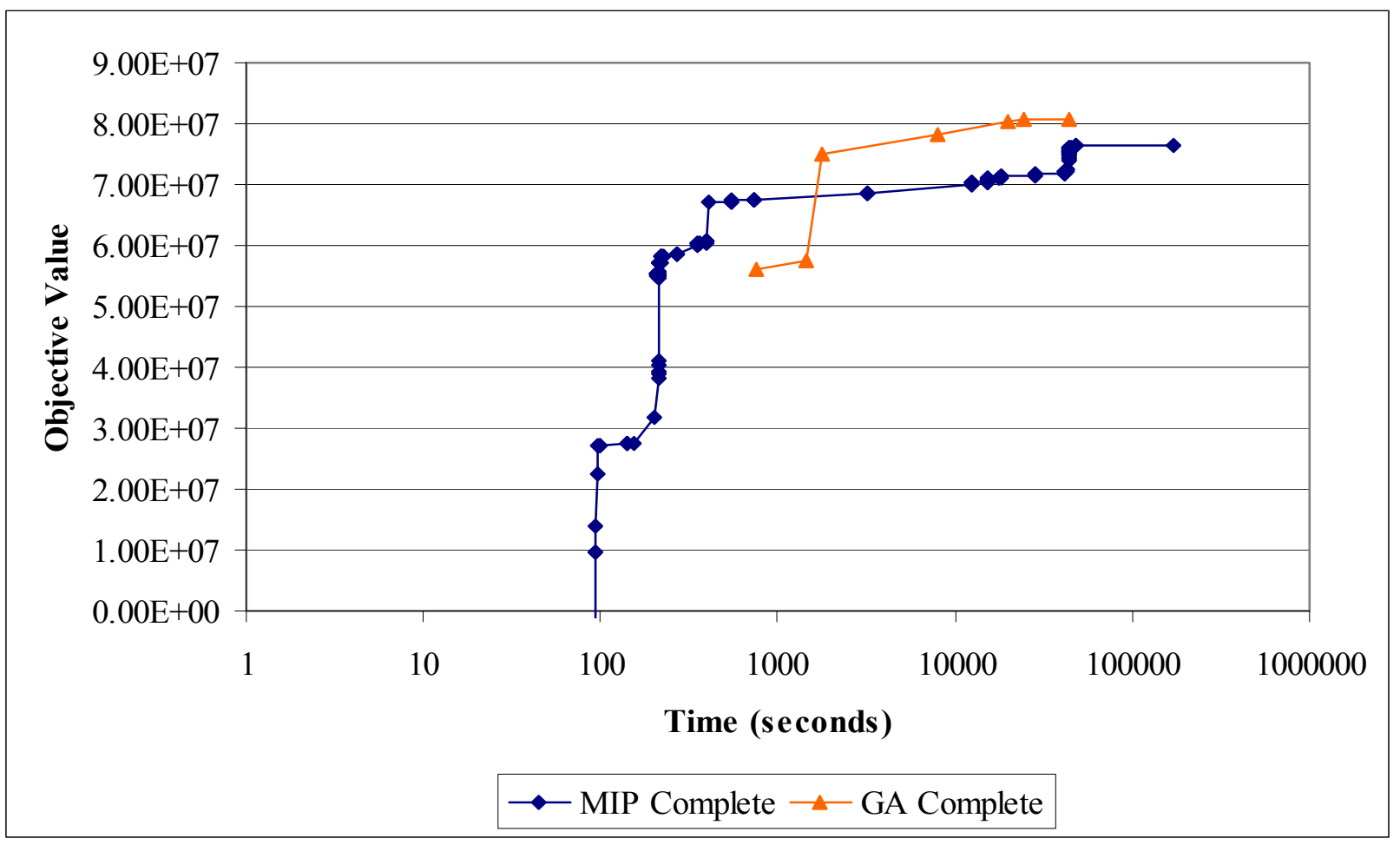

Figure 14: Progress of the MIP and the GA for the complete DFSC model (instance 3) 


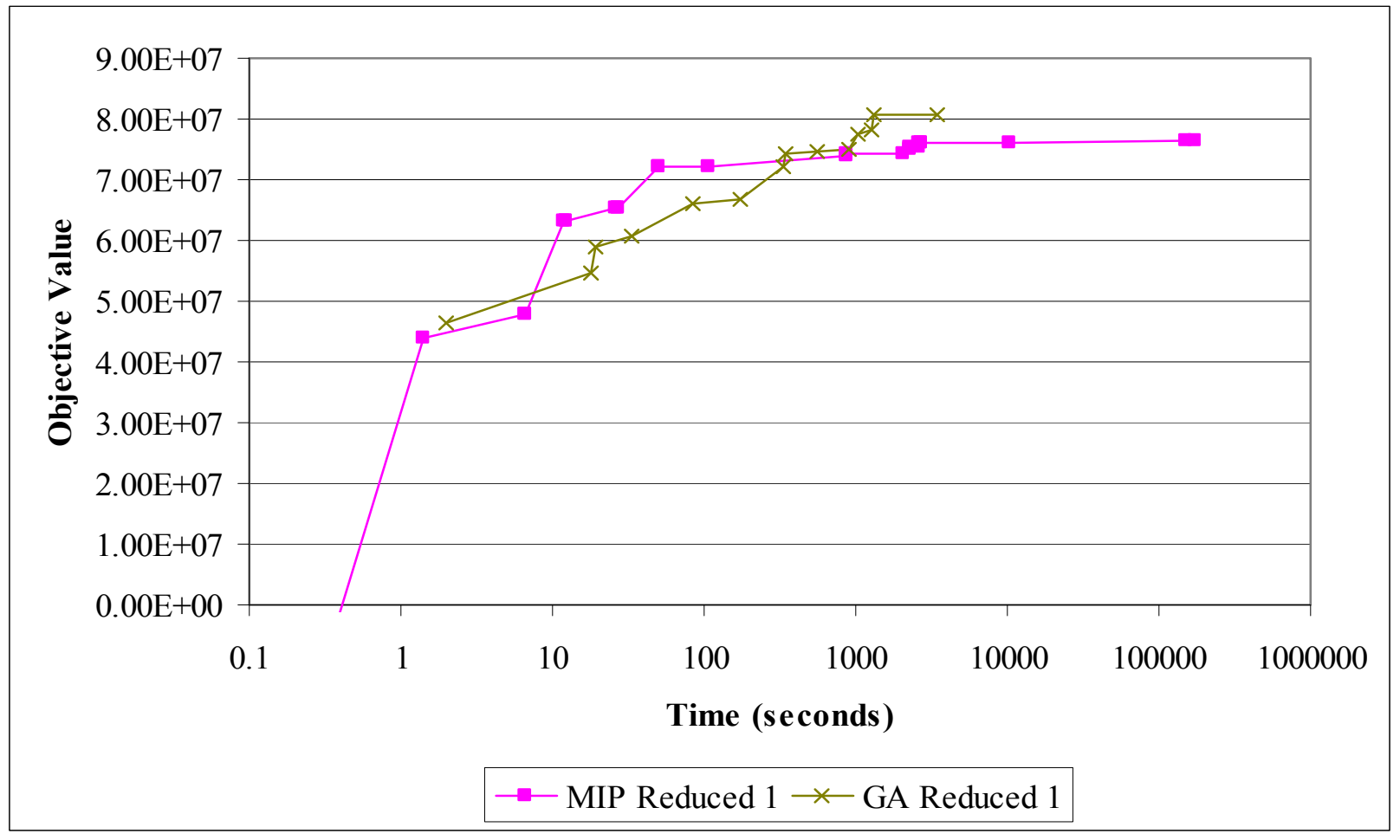

Figure 15: Progress of the MIP and the GA for the reduced DFSC model - 1 (instance 3)

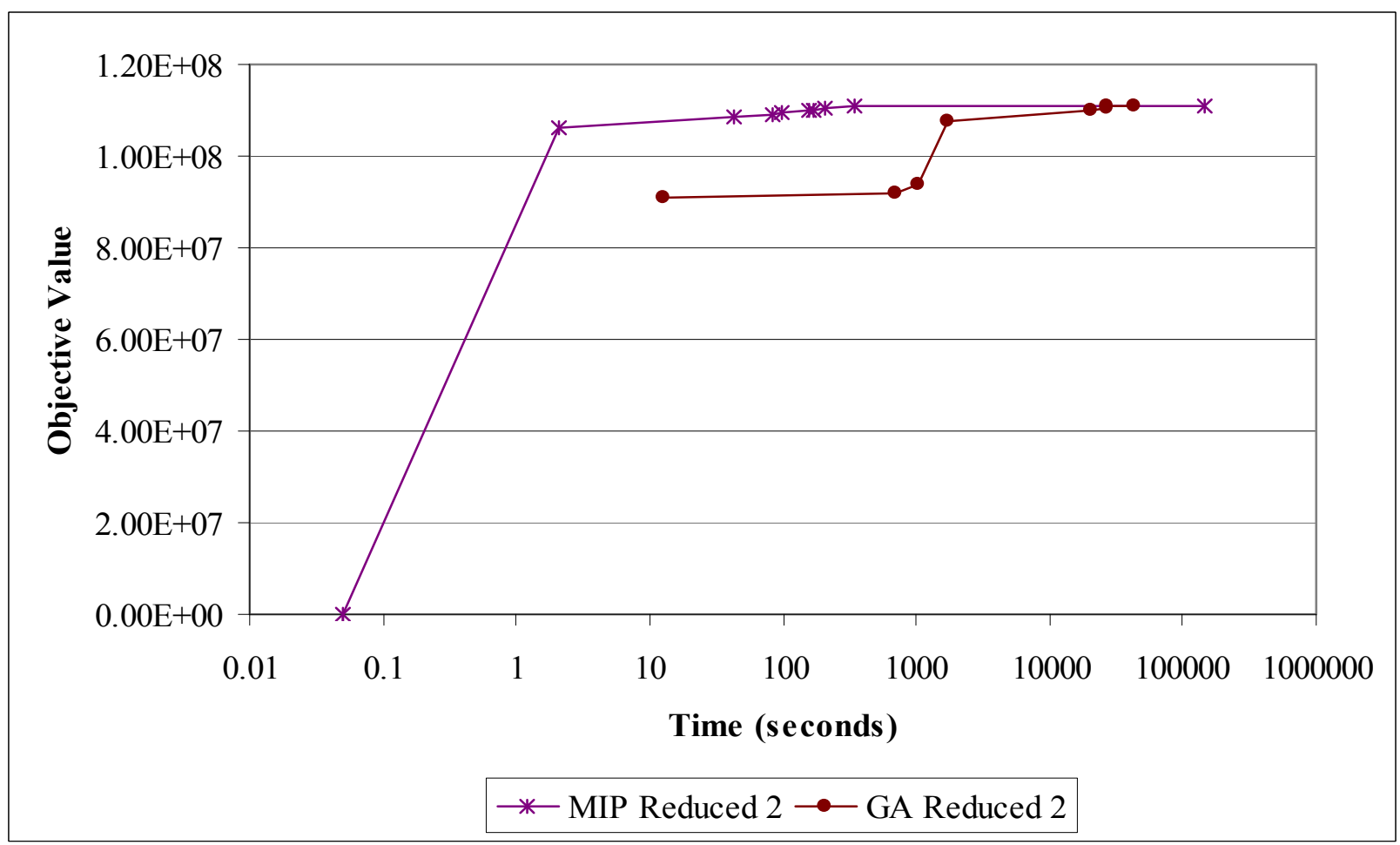

Figure 16: Progress of the MIP and the GA for the reduced DFSC model - 2 (instance 3) 
For the third problem instance, the MIP finds better solutions in the beginning of each run; however the GA finds either better or as good solutions and takes less time in the end. The GA terminates before the MIP for all three models and actually the MIP cannot find as good solutions as the GA within the time limit. For the fourth problem instance, the progress of both algorithms is shown in Figure 17 for the complete DFSC model, in Figure 18 for the reduced DFSC model -1 , and in Figure 19 for the reduced DFSC model -2 .

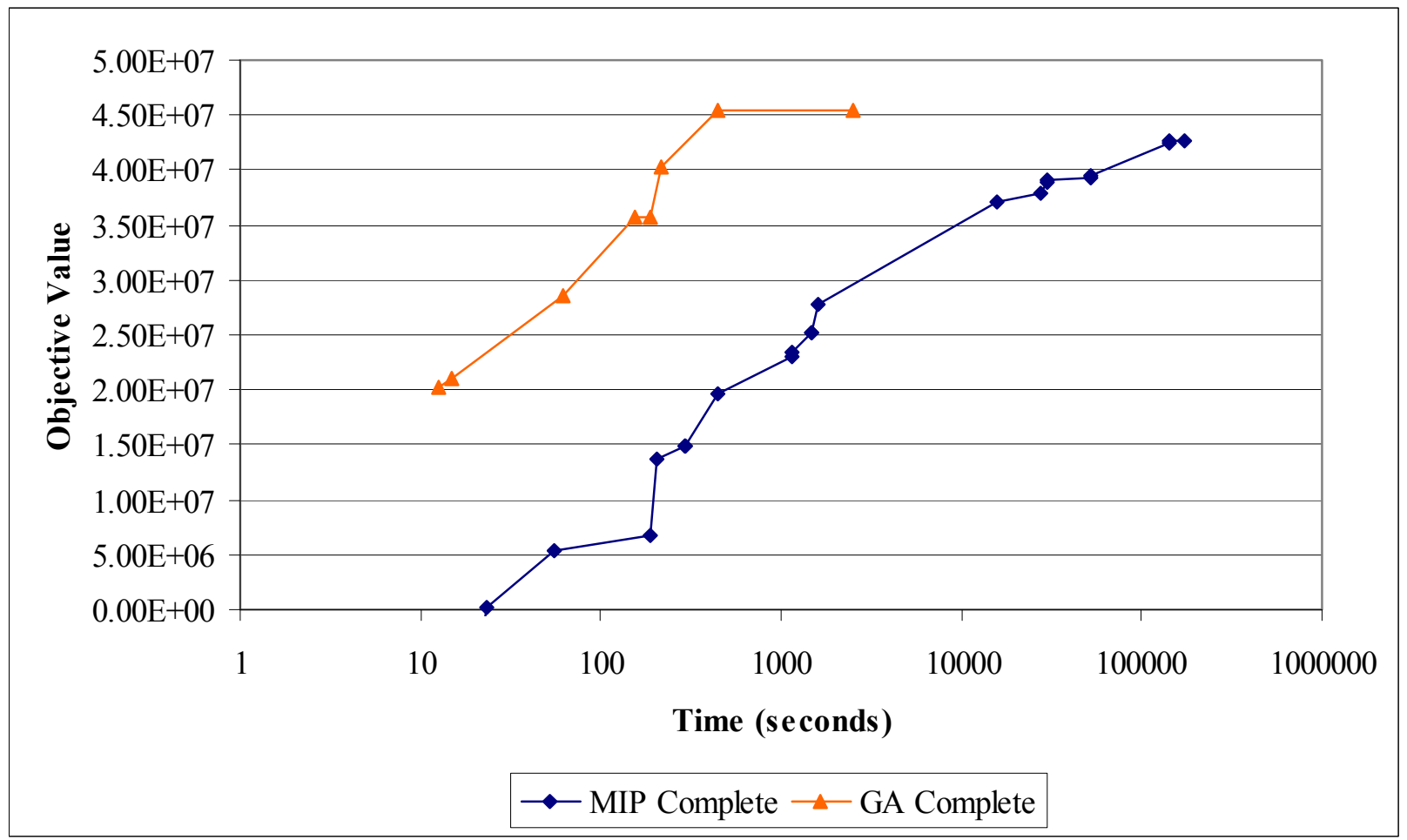

Figure 17: Progress of the MIP and the GA for the complete DFSC model (instance 4) 


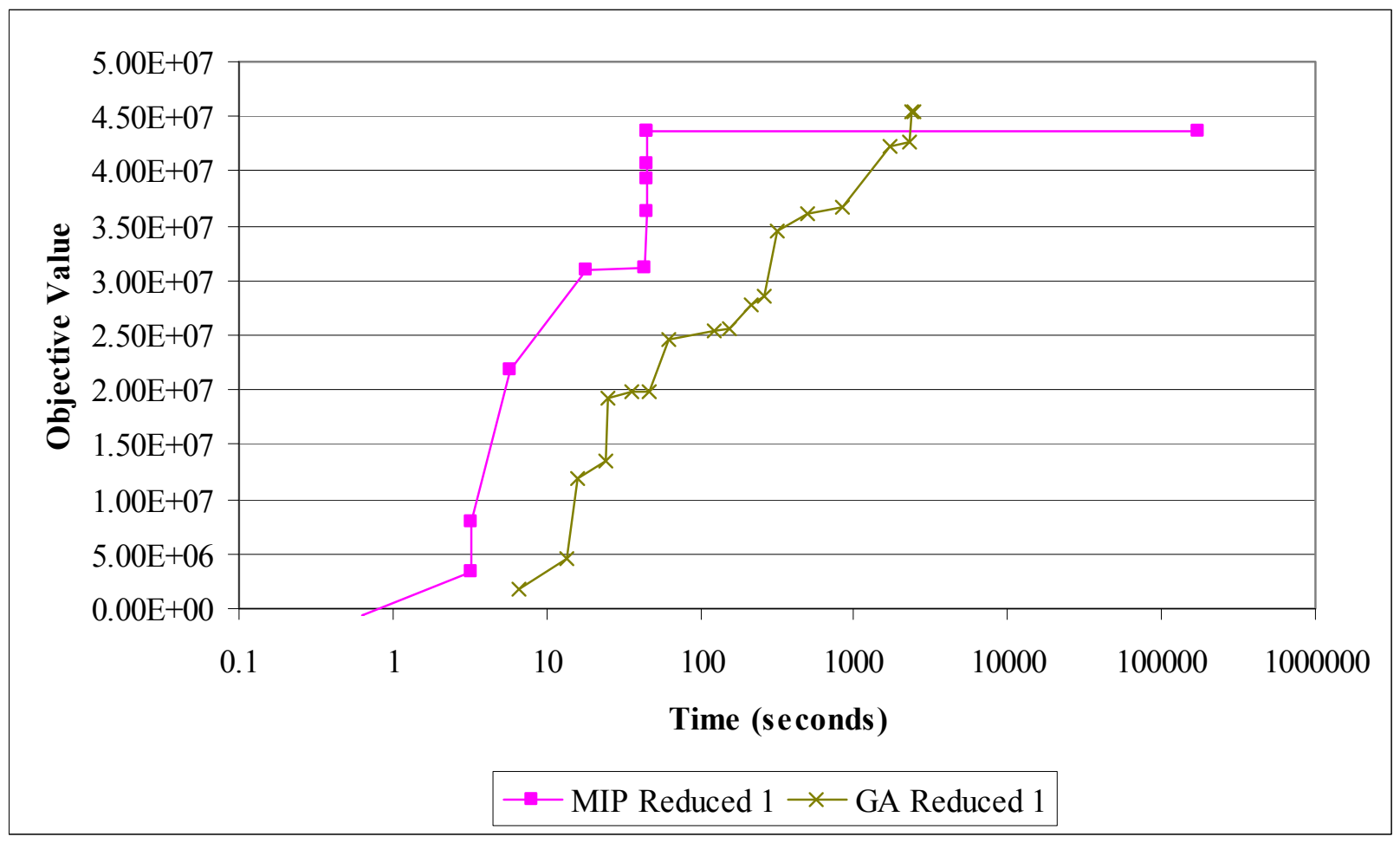

Figure 18: Progress of the MIP and the GA for the reduced DFSC model - 1 (instance 4)

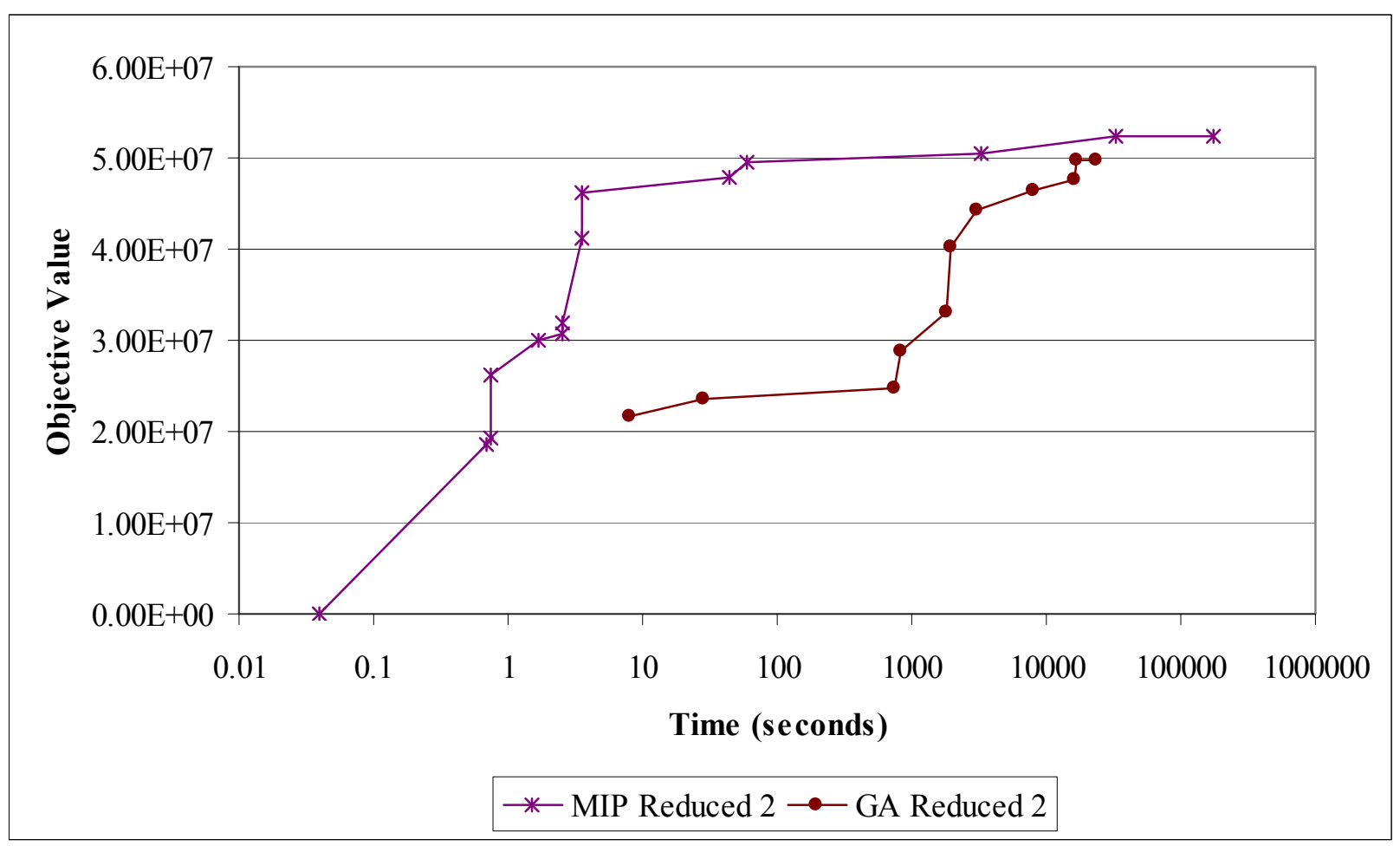

Figure 19: Progress of the MIP and the GA for the reduced DFSC model - 2 (instance 4) 
For the fourth problem instance, the results differ for different models. In the complete DFSC model, the GA outperforms the MIP both in solution quality and time. For the reduced DFSC model - 1, the GA finds a slightly better solution than the MIP; however the MIP finds a very good solution in a much shorter time. For the reduced DFSC model - 2, the MIP outperforms the GA by finding much better solutions very quickly. The GA terminates earlier than MIP due to lack of any improvement in the solution quality before finding a solution as good as MIP did. The progress of both algorithms for the fifth problem instance is shown in Figure 20 for the complete DFSC model, in Figure 21 for the reduced DFSC model - 1, and in Figure 22 for the reduced DFSC model -2 .

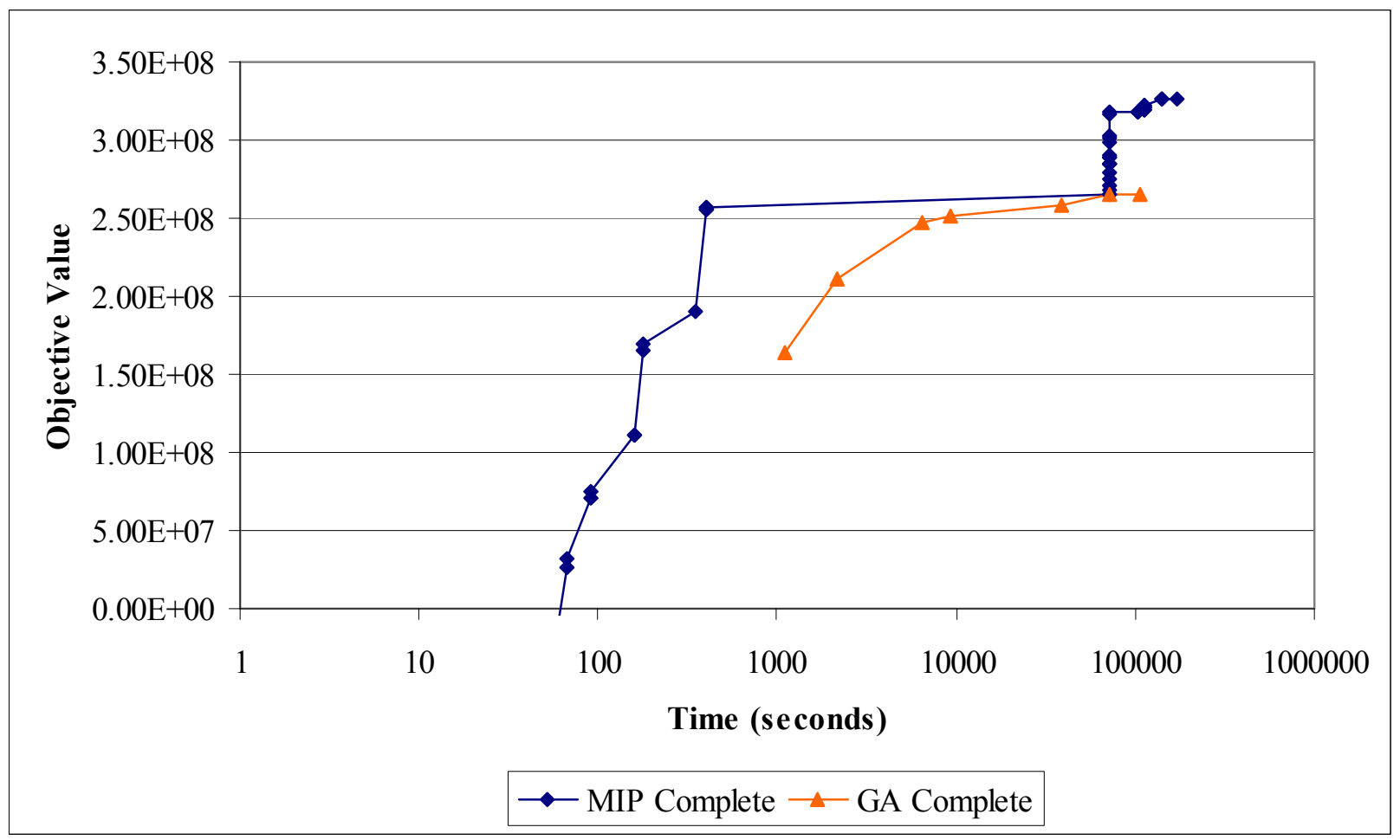

Figure 20: Progress of the MIP and the GA for the complete DFSC model (instance 5) 


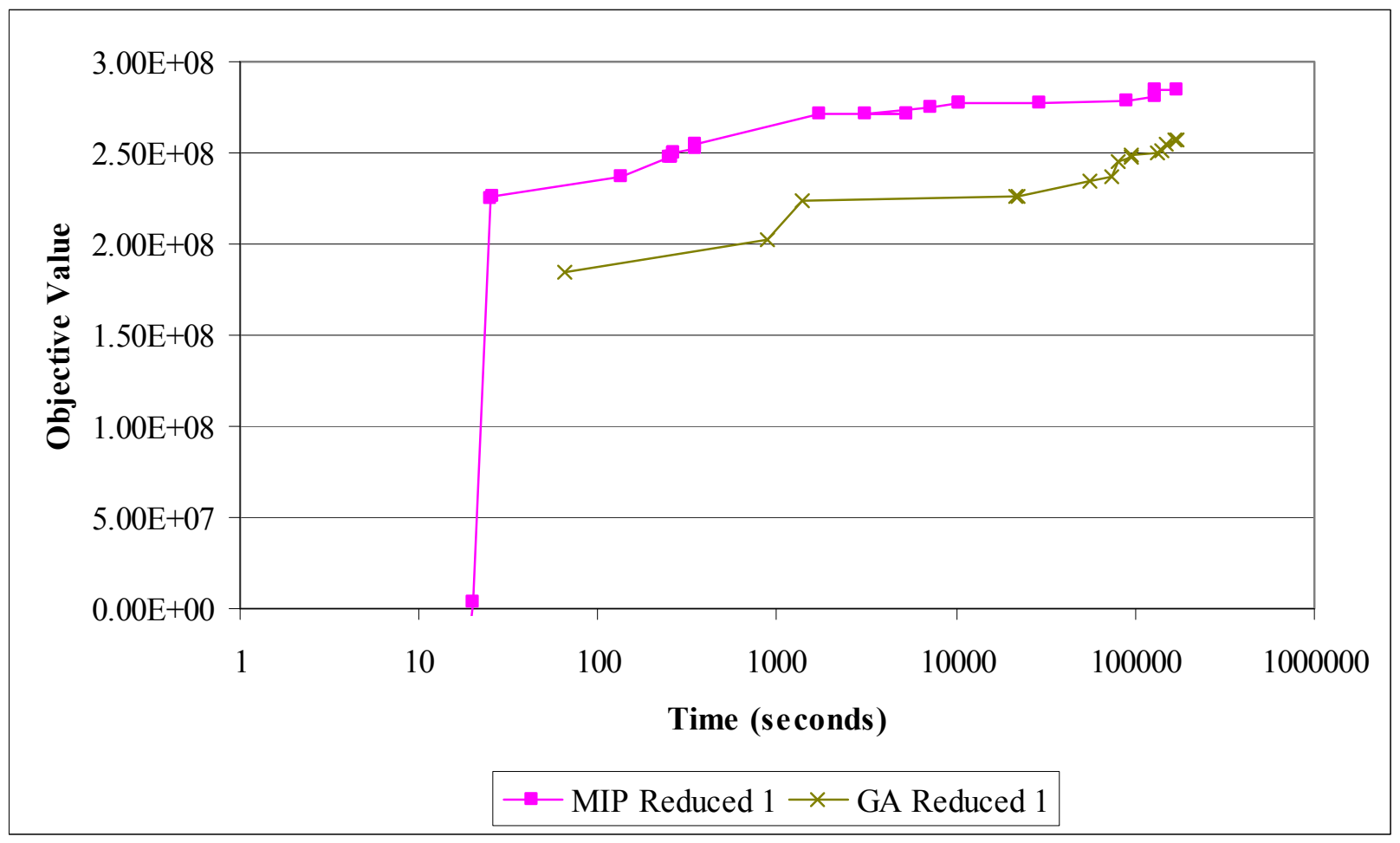

Figure 21: Progress of the MIP and the GA for the reduced DFSC model - 1 (instance 5)

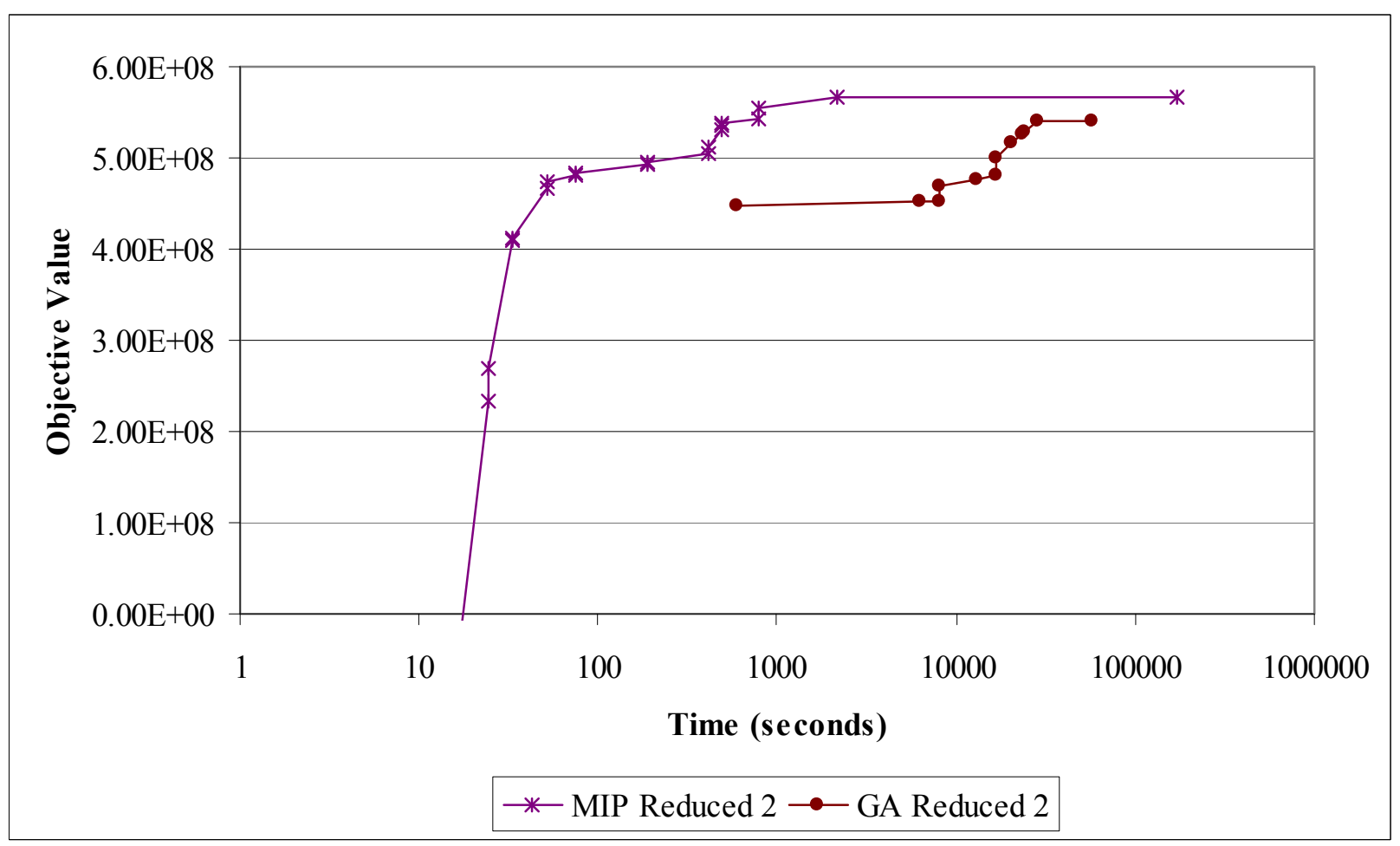

Figure 22: Progress of the MIP and the GA for the reduced DFSC model - 2 (instance 5) 
For the largest problem instance, the MIP procedure terminates due to the time limit before proving the optimality of the solution. However, the GA cannot find a better solution for any of the three models and is dominated by the MIP.

This comparative analysis of the both methods' progressions suggests that neither one of the methods dominates the other for all models or problem sizes. The results show that the GA performs better for the mid-sized problem instances, but it is dominated by the MIP for the smaller instances (due to the terminating conditions) and by the larger instances due to the inherent difficulties that the GA encounters with a large solution space, i.e., a diminishing probability of improving a good solution.

\subsection{PROBLEM AND MODEL VALIDATION}

The DFSC modeling concepts, assumptions, and results are discussed with three different industry experts in order to get industry users' input and validate the models. Some discussions with the industry experts were conducted in the model development phase and their input is incorporated into the models. Further discussions were aimed toward validating the models and assessing the solution quality towards the end of the research. These experts currently work in supply chain planning related tasks at their companies. The first expert works for a U.S.-based company that specializes in sleep and respiratory solutions. The second expert's company manufactures electrical systems and components for automobiles, aircraft, and other industrial equipment and is also based in the U.S. The third expert works in an international company which manufactures electronics and provides electrical engineering solutions to diverse markets. 
The discussions with these experts showed that none of the companies currently employ an integrated DFSC procedure that simultaneously considers product and supply chain design issues in the product design phase. However, both experts from the U.S.-based companies stated that their companies are beginning to utilize more of the simultaneous approach and forsaking the sequential approach. Yet, design teams still maintain primary responsibility for the design process with only partial involvement from supply chain related groups although the involvement by the supply chain groups is increasing. The third company expert stated that they are currently using the sequential approach in all of their product designs.

The U.S.-based companies stated that their primary interest in the supply chain design problems is cost reductions. In terms of the product design, the U.S.-based companies' primary concerns are the satisfaction of the regulations and customer specifications, followed by the improvement of the customer attractiveness of the product. The third company stated that their primary goal both in product and supply chain design is to satisfy specific quality levels required by the customer regardless of its impacts on the cost and other measures. All three experts confirm that the manufacturing, inventory, supply chain network, and transportation costs as well as lead times and supplier capacity limitations are the most important factors for their decisions in the supply chain design decisions. Other variables that they felt were of secondary importance but were not included in these developed models include mainly more qualitative factors such as working history with suppliers, strategic partnerships, number of distinct suppliers, certain risks due to suppliers' processes or location, increasing diversity, and suppliers' company culture. All three experts suggested that although these factors are important, they play a secondary role in the supply selection procedure and are often considered as tie-breaking criteria. This suggests 
that the developed DFSC models would be a valid and useful decision support tool utilizing the most important industry criteria.

In terms of product design, experts suggest that they often change the component designs several times in the product design phase to meet certain specifications and attract more customers or to achieve a better supply chain performance as suggested by the sequential approach. These design changes include, but are not limited to actual geometric design changes, selection of different materials, and using different versions of components such as computer chips. The impacts of these design changes on the demand and customer satisfaction are often assessed by the marketing departments via expert knowledge and forecasting or by customer surveys. Therefore, the industry experts suggest that the developed DFSC concept and models may improve their product design procedures by reducing the number of product design change iterations thus reducing the time to launch the product to the market.

The industry experts suggest that they usually have a considerable amount of information about the potential price levels and demand in the product design phase so the pricing and demand function data requirements of the DFSC models can be satisfied. They also acknowledge that obsolescence plays an important role in their product and supply chain decisions and some level of obsolescence information can be predicted for the DFSC models. Experts stated that cost data such as supplier manufacturing costs and economies of scale information can be gathered with relative ease. However, transportation and inventory costs can only be estimated with a certain level of error due to their highly fluctuating nature. Similarly, suppliers' lead time information may be estimated with some error since it highly depends on the daily operations and capacity utilization levels and may change over time. The initial supply chain network cost concept and its importance is also validated by the industry experts and it is suggested that these 
costs can be estimated since they are required only for the short term. In summary, the data requirements of the DFSC model can be satisfied within a certain level of error, therefore the experts suggested that the DFSC model would add value to their decision support systems.

The experts suggest that removing economies of scale or lead time and inventory concepts from the model in order to be able to solve it or shorten the solution time would have the least significant impact on the quality of the model. Since removing the economies of scale concept (reduced DFSC model - 1) significantly reduces the number of binary variables (and thus the model complexity) and it can easily be approximated via an average unit manufacturing cost value, the experts suggest that it would be reasonable to set this concept aside in order to improve the solution procedure. On the other hand, the removal of lead time and inventory (reduced DFSC model - 2) is a more significant sacrifice making it a secondary choice for the model reduction. The industry experts suggest avoiding some other model reduction suggestions such as removing transportation costs or removing supplier capacity limitations since these concepts add significant value to the models and the data can be estimated with relative accuracy. Price and / or demand decisions, variations due to different product life cycle phases (time periods), supply chain network costs, and product design's impacts on the demand are the most significant value adding and differentiating concepts of the DFSC models and therefore should be kept even if they introduce complexity into the models.

As another suggestion to reduce the model complexity and improve the solution procedures, the experts suggest that the models be solved for the most critical components of the product (as opposed to the whole product) in terms of customer satisfaction, product functionality, and cost and quality impacts. This would reduce the number of components in the model and the number of suppliers to be considered. For example, an analytical analysis such as 
Pareto analysis can be employed to identify the most critical components in terms of their impact on the demand and their challenging requirements on the supply chain configuration. The experts suggest that in most of their products, the number of components that they would consider as critical is less than ten. Therefore, the developed illustrative problem instances can represent a real case and show the complexity of the models even for the instance with few components and suppliers.

In summary, after reviewing the models and illustrations in detail, the industry experts liked the model and the underlying simultaneous optimization approach. They state that the model requires extensive amount of data however the required data would already be gathered for the product design and supplier selections. Therefore, they stated that they would use the developed DFSC models and it would serve as a valuable decision support tool. The industry experts also agreed that the model captures the most important product design and supply chain variables. They also stressed that it would be useful to customize the models into their company's specific needs as further discussed in section 6.2 (extensions for the DFSC models).

\subsection{ANSWERS TO RESEARCH QUESTIONS}

In this research, the performance of the DFSC concept is examined via the stated major research questions. This section provides detailed analysis and answers to the stated research questions. 


\subsubsection{Performance Metrics and Modeling Preferences}

\section{Research Question 1: Which product design / supply chain performance metrics should be included in the model?}

The first research question investigates which product design and supply chain performance metrics should be included in the model in order to keep the models realistic and representative of the real industry problems as well as simple enough to find high quality solutions within acceptable time limits. The reduced DFSC models are used to investigate the performance variations between different modeling preferences. The concepts to be removed from the models are selected based on the model structure (to have significant impacts on the model size and solution performance) and are validated by the industry experts as discussed.

The performance of both solution procedures for the complete and the reduced DFSC models are summarized in Table 20 for the MIP and in Table 21 for the GA.

Table 20: MIP results for the complete and reduced DFSC models

\begin{tabular}{c|rr|rr|rr}
\hline & \multicolumn{2}{|c|}{ Complete DFSC Model } & \multicolumn{2}{c}{ Reduced DFSC Model - 1 } & \multicolumn{2}{c}{ Reduced DFSC Model -2} \\
\hline $\begin{array}{c}\text { Problem } \\
\text { Instance }\end{array}$ & $\begin{array}{c}\text { Optimality } \\
\text { Gap }\end{array}$ & $\begin{array}{c}\text { Solution } \\
\text { Time (sec.) }\end{array}$ & $\begin{array}{c}\text { Optimality } \\
\text { Gap }\end{array}$ & $\begin{array}{c}\text { Solution } \\
\text { Time (sec.) }\end{array}$ & $\begin{array}{c}\text { Optimality } \\
\text { Gap }\end{array}$ & $\begin{array}{c}\text { Solution } \\
\text { Time (sec.) }\end{array}$ \\
\hline 1 & $0.01 \%$ & 150.69 & $0.01 \%$ & 36.89 & $0.01 \%$ & 6.24 \\
2 & $20.61 \%$ & $172,800.00$ & $0.01 \%$ & $48,091.53$ & $0.01 \%$ & $7,023.81$ \\
3 & $2,490.00 \%$ & $172,800.00$ & $15.64 \%$ & $172,800.00$ & $0.01 \%$ & $145,232.95$ \\
4 & $92.01 \%$ & $172,800.00$ & $36.60 \%$ & $172,800.00$ & $49.63 \%$ & $172,800.00$ \\
5 & $96.03 \%$ & $172,800.00$ & $33.11 \%$ & $172,800.00$ & $16.34 \%$ & $172,800.00$ \\
\hline
\end{tabular}


Table 21: GA results for the complete and reduced DFSC models

\begin{tabular}{|c|c|c|c|c|c|c|}
\hline & \multicolumn{2}{|c|}{ Complete DFSC Model } & \multicolumn{2}{|c|}{ Reduced DFSC Model -1} & \multicolumn{2}{|c|}{ Reduced DFSC Model - 2} \\
\hline $\begin{array}{l}\text { Problem } \\
\text { Instance }\end{array}$ & $\begin{array}{c}\text { Optimality } \\
\text { Gap* }\end{array}$ & $\begin{array}{c}\text { Solution } \\
\text { Time (sec.) }\end{array}$ & $\begin{array}{c}\text { Optimality } \\
\text { Gap* }\end{array}$ & $\begin{array}{c}\text { Solution } \\
\text { Time (sec.) }\end{array}$ & $\begin{array}{c}\text { Optimality } \\
\text { Gap* }\end{array}$ & $\begin{array}{c}\text { Solution } \\
\text { Time (sec.) }\end{array}$ \\
\hline 1 & $0.01 \%$ & 300.72 & $0.01 \%$ & 64.75 & $0.01 \%$ & 114.45 \\
\hline 2 & $20.68 \%$ & 291.40 & $0.01 \%$ & 60.13 & $0.01 \%$ & 122.39 \\
\hline 3 & $2,359.39 \%$ & $44,354.28$ & $9.38 \%$ & $3,396.70$ & $0.12 \%$ & $42,608.76$ \\
\hline 4 & $80.11 \%$ & $2,529.32$ & $31.35 \%$ & $2,465.88$ & $56.91 \%$ & $23,767.98$ \\
\hline 5 & $140.62 \%$ & $106,841.33$ & $47.69 \%$ & $172,800.00$ & $22.12 \%$ & $57,246.45$ \\
\hline
\end{tabular}

* The optimality gap values measure the percent difference between the GA solution and the best bound value provided by the MIP for the corresponding MIP model. Note that this table does not compare the MIP and the GA results but it shows the performance variations for different models.

These results clearly show a performance increase for both solution procedures when the complete DFSC is reduced. While the optimality gap is very large for problem instances other than the smallest one (problem instance 1) with the complete model, both algorithms terminate prior to finding an optimal solution due to the time limit or lack of improvements for a specified time. On the other hand, both algorithms progress more rapidly and provide better optimality gap values in a shorter time for the reduced models. Nevertheless, the solution techniques' performances do not provide an apparent conclusion on how they differ for the two reduced models. For the reduced DFSC model -1 , the MIP cannot prove the optimality of the solution within the 48 hour time limit for the three largest problem instances although it improves the optimality gap compared to the complete model. Similarly, the GA terminates in a shorter time and provides a better optimality gap except for the largest instance where it reaches the time limit. For the reduced DFSC model -2 , the optimality gap improvements over the complete model are better for the problem instances 3 and 5 and worse for the instance 4 compared to the first reduced model. Although the reduced DFSC model -1 prunes more binary variables than the second model and therefore is expected to improve both of the solution techniques, the results suggest that the solution performance depends not only on the problem size (e.g., number 
of component design alternatives, number of suppliers) and number of binary variables, but also on the problem instance itself (e.g., Bill of Material complexity, actual cost and lead time values). The differences in the interactions between the model variables due to the different parameter values may help the first reduced model to be solved to a better solution than the second reduced model or vice versa.

In summary, the results suggest that the reduced models can be solved to significantly better solutions (to a lesser optimality gap value) in the same time period or even a shorter time period and therefore become attractive alternatives to the complete DFSC when it cannot be solved or the solution quality is not satisfactory. The loss of detailed information, such as impacts of economies of scale or lead times, may be an acceptable tradeoff to allow for a better solution performance. As industry experts agree, the economies of scale information can be approximated by an average cost value and then can be evaluated after the model is solved and the final product design and price are fixed. On the other hand, when the first reduced model solutions are not satisfactory, lead time and inventory information can be removed as in the second reduced model. However, the loss of this information is more significant in this model as these concepts cannot be approximated with any other parameters remaining in the model. Therefore, the second reduced model is only preferred when the complete and the first reduced models cannot be solved. However, when the second reduced model is utilized, the results should be further analyzed qualitatively by investigating the impacts of the simplifications to the model.

In order to test the changes in the model solution and objective value with respect to the parameter estimation errors, a sensitivity analysis is conducted. For these tests, a new problem instance based on a simple cordless phone example is created. The cordless phone in this 
example is assumed to consist of four components, namely a housing, battery, printed circuit board, and antenna. This example is modeled with five components including the final assembly of the cordless phone itself as another component. Although this final assembly is regarded as a standalone component in order to capture supplier selection and cost aspects, it does not have any design alternatives nor any customer satisfaction value. The remaining four components have two design alternatives each and the customer satisfaction value of these designs change over time to reflect obsolescence. There are four time periods in this example representing four main product life cycle phases as described before. The customer satisfaction values of each component over four time periods are given in Table 22. For this problem, five suppliers are considered, with the first supplier being the final manufacturer and the only supplier available for the final assembly. 
Table 22: Customer satisfaction values of component design alternatives - cordless phone

\begin{tabular}{|c|c|c|c|c|c|}
\hline Total Value & $\begin{array}{c}\text { Appearance } \\
40.0 \% \\
\end{array}$ & $\begin{array}{c}\text { Battery Life } \\
30.0 \% \\
\end{array}$ & $\begin{array}{c}\text { Signal } \\
\text { Quality } \\
15.0 \%\end{array}$ & $\begin{array}{c}\text { Dialing } \\
\text { Functions } \\
15.0 \% \\
\end{array}$ & $\begin{array}{c}\text { Total } \\
100.0 \%\end{array}$ \\
\hline \multicolumn{6}{|l|}{ Component } \\
\hline $\begin{array}{l}\text { Cordless } \\
\text { Phone }\end{array}$ & $0.0 \%$ & $0.0 \%$ & $0.0 \%$ & $0.0 \%$ & $0.0 \%$ \\
\hline \multicolumn{6}{|l|}{ Housing } \\
\hline Design 1 & $\begin{array}{l}30.0 \%-30.0 \%- \\
30.0 \%-30.0 \%\end{array}$ & & & $\begin{array}{l}4.0 \%-4.0 \%- \\
4.0 \%-4.0 \%\end{array}$ & $\begin{array}{l}34.0 \%-34.0 \%- \\
34.0 \%-34.0 \%\end{array}$ \\
\hline Design 2 & $\begin{array}{l}24.0 \%-24.0 \%- \\
21.0 \%-18.0 \% \\
\end{array}$ & & & $\begin{array}{l}3.6 \%-3.2 \%- \\
3.2 \%-2.8 \%\end{array}$ & $\begin{array}{l}27.6 \%-27.2 \%- \\
24.2 \%-20.8 \% \\
\end{array}$ \\
\hline \multicolumn{6}{|l|}{ Battery } \\
\hline Lithium Ion & & $\begin{array}{l}25.0 \%-25.0 \%- \\
25.0 \%-25.0 \%\end{array}$ & $\begin{array}{l}1.0 \%-1.0 \%- \\
1.0 \%-1.0 \%\end{array}$ & & $\begin{array}{l}26.0 \%-26.0 \%- \\
26.0 \%-26.0 \%\end{array}$ \\
\hline $\mathrm{Ni}-\mathrm{Cd}$ & & \begin{tabular}{|l}
$20.0 \%-17.5 \%-$ \\
$12.5 \%-7.5 \%$ \\
\end{tabular} & $\begin{array}{l}0.9 \%-0.9 \%- \\
0.9 \%-0.9 \%\end{array}$ & & \begin{tabular}{|l}
$20.9 \%-18.4 \%-$ \\
$13.4 \%-8.4 \%$ \\
\end{tabular} \\
\hline \multicolumn{6}{|l|}{$\begin{array}{l}\text { Printed } \\
\text { Circuit Board }\end{array}$} \\
\hline Design 1 & & $\begin{array}{l}5.0 \%-5.0 \%- \\
5.0 \%-5.0 \%\end{array}$ & $\begin{array}{l}6.0 \%-6.0 \%- \\
6.0 \%-6.0 \%\end{array}$ & $\begin{array}{l}11.0 \%-11.0 \%- \\
11.0 \%-11.0 \%\end{array}$ & $\begin{array}{l}22.0 \%-22.0 \%- \\
22.0 \%-22.0 \%\end{array}$ \\
\hline Design 2 & & $\begin{array}{l}4.5 \%-4.5 \%- \\
4.5 \%-4.5 \%\end{array}$ & $\begin{array}{l}4.8 \%-4.8 \%- \\
4.8 \%-4.8 \% \\
\end{array}$ & $\begin{array}{l}8.8 \%-7.7 \%- \\
7.7 \%-5.5 \% \\
\end{array}$ & $\begin{array}{l}18.1 \%-17.0 \%- \\
17.0 \%-14.8 \% \\
\end{array}$ \\
\hline \multicolumn{6}{|l|}{ Antenna } \\
\hline Design 1 & $\begin{array}{l}10.0 \%-10.0 \%- \\
10.0 \%-10.0 \%\end{array}$ & & $\begin{array}{l}8.0 \%-8.0 \%- \\
8.0 \%-8.0 \%\end{array}$ & & $\begin{array}{l}18.0 \%-18.0 \%- \\
18.0 \%-18.0 \%\end{array}$ \\
\hline Design 2 & \begin{tabular}{|l|}
$9.0 \%-9.0 \%-$ \\
$9.0 \%-9.0 \%$ \\
\end{tabular} & & $\begin{array}{l}5.6 \%-5.6 \%- \\
5.6 \%-5.6 \%\end{array}$ & & $\begin{array}{l}14.6 \%-14.6 \%- \\
14.6 \%-14.6 \%\end{array}$ \\
\hline
\end{tabular}

* Four different values in the table correspond to component design values for four time periods. The time periods are ordered as $\mid$ time period 1 - time period $2 \mid$ in the table.

|time period 3 - time period $4 \mid$ 
To test differences in the objective function and solution variables, first the initial problem with the original parameter values (base case) is solved. Next, to represent estimation errors, important parameter values are increased or decreased by $10 \%$ separately from the base case. The tested parameters include supplier capacities (Capacity ${ }_{i j \alpha_{i} t}{ }^{1,2}$ ), unit manufacturing costs at suppliers $\left(c_{i j \alpha_{i} t}{ }^{l, 2}\right)$, production times at suppliers $\left(p t_{i j j_{i}}\right)$, supply chain network costs $\left(\right.$ Netwco $\left._{j l}\right)$, transportation costs $\left(\operatorname{Tportcost}_{j k l \alpha_{k}}\right)$, and demand function coefficients $\left(\beta_{1}, \beta_{2}\right)$. Table 23 shows the objective value of each scenario and whether the variables of supplier selection (supply chain design), component alternatives selection (product design), and pricing differ from the base case. All tests are conducted by using the MIP solution procedure by solving the complete DFSC model therefore these tests capture all the described concepts and the solutions are proven to be optimal. It should be noted that the aim of these tests is not to show how the actual solution values change but rather to understand how much impact these estimation errors might have on the primary product and supply chain designs. Therefore, the changes in the variables from the base case primarily investigate whether another design or another supplier for a component is selected and if the pricing decisions are changed. The pricing decisions are important since price has a significant impact on the demand and may influence selection of different product or supply chain designs. 
Table 23: Parameter estimation error test results

\begin{tabular}{|c|c|c|c|c|c|}
\hline \multirow[b]{2}{*}{ Scenario } & \multirow{2}{*}{$\begin{array}{c}\text { Objective } \\
\text { Value (profit) } \\
\text { (\$) }\end{array}$} & \multicolumn{3}{|c|}{$\begin{array}{l}\text { Change from the Base } \\
\text { Case }\end{array}$} & \multirow{2}{*}{$\begin{array}{l}\text { Solution } \\
\text { Time } \\
\text { (sec.) }\end{array}$} \\
\hline & & $\begin{array}{l}\text { Product } \\
\text { Design }\end{array}$ & $\begin{array}{c}\text { Price } \\
(\$)\end{array}$ & $\begin{array}{l}\text { Supply } \\
\text { Chain } \\
\text { Design }\end{array}$ & \\
\hline Base Case & $85,462,662$ & - & - & - & $16,606.92$ \\
\hline Supplier capacity $(+10 \%)$ & $88,765,433 \uparrow$ & Yes & No & Yes & $2,954.48$ \\
\hline Supplier capacity $(-10 \%)$ & $81,205,455 \downarrow$ & Yes & No & No & $73,292.46$ \\
\hline Unit manufacturing cost $(+10 \%)$ & $82,818,261 \downarrow$ & No & No & Yes & $21,108.20$ \\
\hline Unit manufacturing cost $(-10 \%)$ & $88,211,200 \uparrow$ & Yes & No & Yes & $16,257.85$ \\
\hline Supplier production time $(+10 \%)$ & $83,693,537 \downarrow$ & No & No & Yes & $38,170.30$ \\
\hline Supplier production time $(-10 \%)$ & $87,240,959 \uparrow$ & Yes & No & Yes & $27,672.56$ \\
\hline Supply chain network cost $(+10 \%)$ & $83,955,662 \downarrow$ & No & No & No & $14,514.36$ \\
\hline Supply chain network cost $(-10 \%)$ & $86,969,662 \uparrow$ & No & No & No & $15,995.99$ \\
\hline Transportation cost $(+10 \%)$ & $84,263,290 \downarrow$ & No & No & No & $45,424.48$ \\
\hline Transportation cost (-10\%) & $86,662,033 \uparrow$ & No & No & No & $18,484.96$ \\
\hline Demand function coefficients $(+10 \%)$ & $100,850,314 \uparrow$ & Yes & No & Yes & $32,361.29$ \\
\hline Demand function coefficients $(-10 \%)$ & $68,012,999 \downarrow$ & Yes & No & Yes & $17,516.67$ \\
\hline
\end{tabular}

The results suggest that for this problem instance, underestimating the cost parameter values or overestimating the supplier capacities and the demand function coefficients results in lower profits due to increases in the realized costs or unexpected supplier capacity limitations and lower realized demand. Estimation errors in the opposite directions result in higher profits due to lower supply chain costs, higher demand, or more supplier production capacity.

A $10 \%$ estimation error in the supply chain network costs or in the transportation costs do not change the preferred product design and supplier selection. However, estimation errors on other parameters have different consequences and depend on the actual problem data and interactions between the variables. For example, the demand function coefficients have a significant impact on the profit value since they directly impact the demand and revenue rather than the supply chain costs. An interesting result of these tests is that none of the scenarios resulted in a change in the optimal price levels. 
Since the product and supply chain design decisions are made before the parameter errors are realized in a real life situation, additional sensitivity analysis is performed to further examine the impact of these errors by further illustrating such an issue. In this case, instead of optimizing the problem from the beginning with the new parameter values for each scenario, the optimal solution for the base case is used with the new parameter values. The same product design selections, supplier selections (thus supply chain network), and price values that are optimal for the base case are kept the same for each scenario. The remaining decisions including production and transportation quantities are optimized again. Table 24 shows the results of these tests. In the second column of this table, the profit values for the scenarios are shown where the base case scenario decisions are maintained. In the third column, the re-optimized profit results for each scenario are given. It should be noted that these profit values are the same results as shown in Table 23. The difference in the profit values when the base case solution is used and when the problems are re-optimized is shown in the fourth column. 
Table 24: Parameter estimation error test results by using the base case solution

\begin{tabular}{l|c|c|c}
\hline \multicolumn{1}{c|}{ Scenario } & $\begin{array}{c}\text { Objective } \\
\text { Value with } \\
\text { Base Case } \\
\text { Solution (\$) }\end{array}$ & $\begin{array}{c}\text { Objective Value } \\
\text { with } \\
\text { Re-optimized } \\
\text { Solution (\$) }\end{array}$ & $\begin{array}{c}\text { Change in the } \\
\text { Objective Value } \\
\text { with } \\
\text { Re-optimization (\$) }\end{array}$ \\
\hline Base Case & $85,462,662$ & $85,462,662$ & - \\
Supplier capacity (+10\%) & $86,398,935$ & $88,765,433$ & $2,366,498$ \\
Supplier capacity (-10\%) & $81,069,890$ & $81,205,455$ & 135,565 \\
Unit manufacturing cost (+10\%) & $82,818,261$ & $82,818,261$ & - \\
Unit manufacturing cost (-10\%) & $88,107,062$ & $88,211,200$ & 104,138 \\
Supplier production time (+10\%) & $83,693,537$ & $83,693,537$ & - \\
Supplier production time (-10\%) & $87,218,392$ & $87,240,959$ & 22,567 \\
Supply chain network cost (+10\%) & $83,955,662$ & $83,955,662$ & - \\
Supply chain network cost (-10\%) & $86,969,662$ & $86,969,662$ & - \\
Transportation cost (+10\%) & $84,263,290$ & $84,263,290$ & - \\
Transportation cost (-10\%) & $86,662,033$ & $86,662,033$ & - \\
Demand function coefficients (+10\%) & $93,583,581$ & $100,850,314$ & $7,266,733$ \\
Demand function coefficients (-10\%) & $65,926,952$ & $68,012,999$ & $2,086,047$ \\
\hline
\end{tabular}

The results show that the re-optimization improves the objective values for some scenarios. However, since re-optimization cannot occur in real life, changes in the profit values while maintaining the decisions made in the base case provide important clues of the impact of the parameter estimation errors. The results showed that the increased and reduced supplier capacities, lower manufacturing costs and production times, and changes in the demand function coefficients have resulted in lower profits compared to the re-optimization results. This suggests that these parameter estimation errors have significant impact on the profit and if the base case decisions could be altered later in the product life cycle phases, higher profit values could be obtained. For other scenarios, base case decisions resulted in the same profit value as the reoptimization suggesting that the decisions based on the erroneous estimations for these parameters would still be optimal when true parameter values are observed. 
Nevertheless, these test results only provide a general idea about the significance of the impacts of these parameter estimation errors. Yet, the impact of these parameter estimation errors may be different for other problem instances depending on the interactions between these parameters in the model. Another important result of these tests is that the solution time for these problems may significantly change based on the actual parameter values. For this particular problem instance, the solution time for different scenarios changed significantly from approximately 45 minutes to 20 hours. The results suggest that supplier capacity values have the most significant impact on the solution time. Increased supplier capacities significantly reduced the solution time by providing enough capacity from the selected suppliers where reduced capacities lengthen the solution time since the production amounts need to be distributed among different suppliers for the optimal allocation.

\subsubsection{Simultaneous versus Sequential Approach}

\section{Research Question 2: How do the performance metrics differ for the product design and the associated supply chain for the simultaneous and sequential approaches?}

The second research question investigates how sequential and simultaneous approaches impact the product and supply chain design performance metrics. In order to investigate this research question, two problem instances are tested since this research question aims to test the main contribution of this dissertation and further insight from additional problem instances is important. In addition to the previously described cordless phone example, a new problem instance which is based on a desktop computer is introduced. In this example, the final product is considered as a standalone component with no design alternatives and no design value. The

remaining five components are the motherboard \& CPU, memory, hard disk, monitor, and 
peripherals. The components' design values are selected to be diminishing over four different time periods to capture obsolescence impacts similar to the cordless phone example. The customer satisfaction values of each component over four time periods are given in Table 25. For this problem, seven suppliers are considered, as the first supplier being the final manufacturer and the only supplier available for the final assembly. 
Table 25: Customer satisfaction values of component design alternatives - desktop computer

\begin{tabular}{|c|c|c|c|c|c|c|}
\hline Total Value & $\begin{array}{c}\text { Perform- } \\
\text { ance } \\
50.0 \%\end{array}$ & $\begin{array}{c}\text { Ease of } \\
\text { Use } \\
13.0 \%\end{array}$ & $\begin{array}{c}\text { Storage } \\
\text { Capacity } \\
10.0 \%\end{array}$ & $\begin{array}{c}\text { Display } \\
\text { Quality } \\
17.0 \%\end{array}$ & $\begin{array}{c}\text { Appear- } \\
\text { ance } \\
10.0 \%\end{array}$ & $\begin{array}{c}\text { Total } \\
100.0 \%\end{array}$ \\
\hline \multicolumn{7}{|l|}{ Component } \\
\hline $\begin{array}{l}\text { Desktop } \\
\text { Computer }\end{array}$ & $0.0 \%$ & $0.0 \%$ & $0.0 \%$ & $0.0 \%$ & & $0.0 \%$ \\
\hline \multicolumn{7}{|l|}{$\begin{array}{l}\text { Motherboard } \\
\& \mathrm{CPU}\end{array}$} \\
\hline $2.5 \mathrm{GHz}$ & $\begin{array}{l}35.0 \%-35.0 \% \\
35.0 \%-35.0 \%\end{array}$ & & & $\begin{array}{l}5.0 \%-5.0 \%- \\
5.0 \%-5.0 \%\end{array}$ & & $\begin{array}{l}40.0 \%-40.0 \% \\
40.0 \%-40.0 \%\end{array}$ \\
\hline $2.0 \mathrm{GHz}$ & $\begin{array}{l}30.0 \%-25.0 \% \\
20.0 \%-10.0 \% \\
\end{array}$ & & & $\begin{array}{l}4.0 \%-3.0 \%- \\
2.0 \%-2.0 \%\end{array}$ & & $\begin{array}{l}34.0 \%-28.0 \%- \\
22.0 \%-12.0 \%\end{array}$ \\
\hline \multicolumn{7}{|l|}{ Memory } \\
\hline $1 \mathrm{~GB}$ & $\begin{array}{l}10.0 \%-10.0 \%- \\
10.0 \%-10.0 \%\end{array}$ & & & $\begin{array}{l}4.0 \%-4.0 \%- \\
4.0 \%-4.0 \%\end{array}$ & & $\begin{array}{l}14.0 \%-14.0 \%- \\
14.0 \%-14.0 \%\end{array}$ \\
\hline $512 \mathrm{MB}$ & \begin{tabular}{|l|}
$7.0 \%-6.0 \%-$ \\
$4.0 \%-2.0 \%$
\end{tabular} & & & $\begin{array}{l}3.0 \%-2.0 \%- \\
2.0 \%-1.0 \%\end{array}$ & & $\begin{array}{l}10.0 \%-8.0 \%- \\
6.0 \%-3.0 \%\end{array}$ \\
\hline \multicolumn{7}{|l|}{ Hard Disk } \\
\hline $120 \mathrm{~GB}$ & $\begin{array}{l}5.0 \%-5.0 \%- \\
5.0 \%-5.0 \%\end{array}$ & & $\begin{array}{l}8.0 \%-8.0 \% \\
8.0 \%-8.0 \%\end{array}$ & & & $\begin{array}{l}13.0 \%-13.0 \%- \\
13.0 \%-13.0 \%\end{array}$ \\
\hline $60 \mathrm{~GB}$ & $\begin{array}{l}4.0 \%-4.0 \%- \\
3.0 \%-1.0 \% \\
\end{array}$ & & $\begin{array}{l}6.0 \%-6.0 \% \\
4.0 \%-1.0 \%\end{array}$ & & & $\begin{array}{l}10.0 \%-10.0 \%{ }^{-} \\
7.0 \%-2.0 \% \\
\end{array}$ \\
\hline \multicolumn{7}{|l|}{ Monitor } \\
\hline $19 "$ LCD & & $\begin{array}{l}3.0 \%-3.0 \%- \\
3.0 \%-3.0 \%\end{array}$ & & $\begin{array}{l}8.0 \%-8.0 \%- \\
8.0 \%-8.0 \%\end{array}$ & $\begin{array}{l}5.0 \%-5.0 \%- \\
5.0 \%-5.0 \%\end{array}$ & $\begin{array}{l}16.0 \%-16.0 \%- \\
16.0 \%-16.0 \%\end{array}$ \\
\hline 19” CRT & & $\begin{array}{l}2.0 \%-2.0 \%- \\
1.0 \%-0.0 \%\end{array}$ & & $\begin{array}{l}6.0 \%-5.0 \%- \\
3.0 \%-1.0 \% \\
\end{array}$ & $\begin{array}{l}2.0 \%-2.0 \%- \\
0.0 \%-0.0 \%\end{array}$ & $\begin{array}{l}10.0 \%-9.0 \%- \\
4.0 \%-1.0 \%\end{array}$ \\
\hline \multicolumn{7}{|l|}{ Peripherals } \\
\hline Advanced & & $\begin{array}{l}10.0 \%-10.0 \% \\
10.0 \%-10.0 \%\end{array}$ & $\begin{array}{l}2.0 \%-2.0 \%- \\
2.0 \%-2.0 \%\end{array}$ & & $\begin{array}{l}5.0 \%-5.0 \%- \\
5.0 \%-5.0 \%\end{array}$ & $\begin{array}{l}17.0 \%-17.0 \%- \\
17.0 \%-17.0 \%\end{array}$ \\
\hline Standard & & $\begin{array}{l}8.0 \%-8.0 \%- \\
4.0 \%-4.0 \% \\
\end{array}$ & $\begin{array}{l}1.0 \%-1.0 \% \\
0.0 \%-0.0 \%\end{array}$ & & $\begin{array}{l}4.0 \%-3.0 \%- \\
3.0 \%-2.0 \%\end{array}$ & $\begin{array}{l}13.0 \%-12.0 \%- \\
7.0 \%-6.0 \% \\
\end{array}$ \\
\hline
\end{tabular}

* Four different values in the table correspond to component design values for four time periods. The time periods are ordered as |time period 1 - time period 2| in the table.

|time period 3 - time period $4 \mid$ 
In order to evaluate the differences between the sequential and simultaneous approaches as stated in this research question, each problem instance (cordless phone and desktop computer examples) is first solved with the simultaneous approach. Subsequently, each problem is solved via the sequential approach. Since the product design value should be as high as possible according to the sequential approach (as the design teams would prefer higher design values in a sequential approach), the initial solution has been based on selecting the best design alternatives for each component which would maximize the demand. After the complete DFSC model is solved for this scenario, each component design is changed to a less preferred alternative following a minimum loss of design value rule. This procedure aims to capture a real case where a design team would compromise from a component's design (to potentially reduce SC costs) which would cause the minimum loss of total product design value, that is, the component with the lowest impact on the demand. In order to truly capture the impact of the design change on the demand, the design value loss is multiplied by the time multipliers which are used in the complete DFSC model to represent each product life cycle phase's impact on the demand. For example, assume that the first two time multipliers which correspond to introduction and growth phases of a product's life cycle are equal to 1 and 2, respectively. This means that for the same demand parameters (product design and price); the demand in the growth phase would be twice the demand in the introduction phase. Therefore, the impact of the design value changes would be more on the growth phase compared to the introduction phase. The described sequential approach procedure is depicted in Figure 23. 


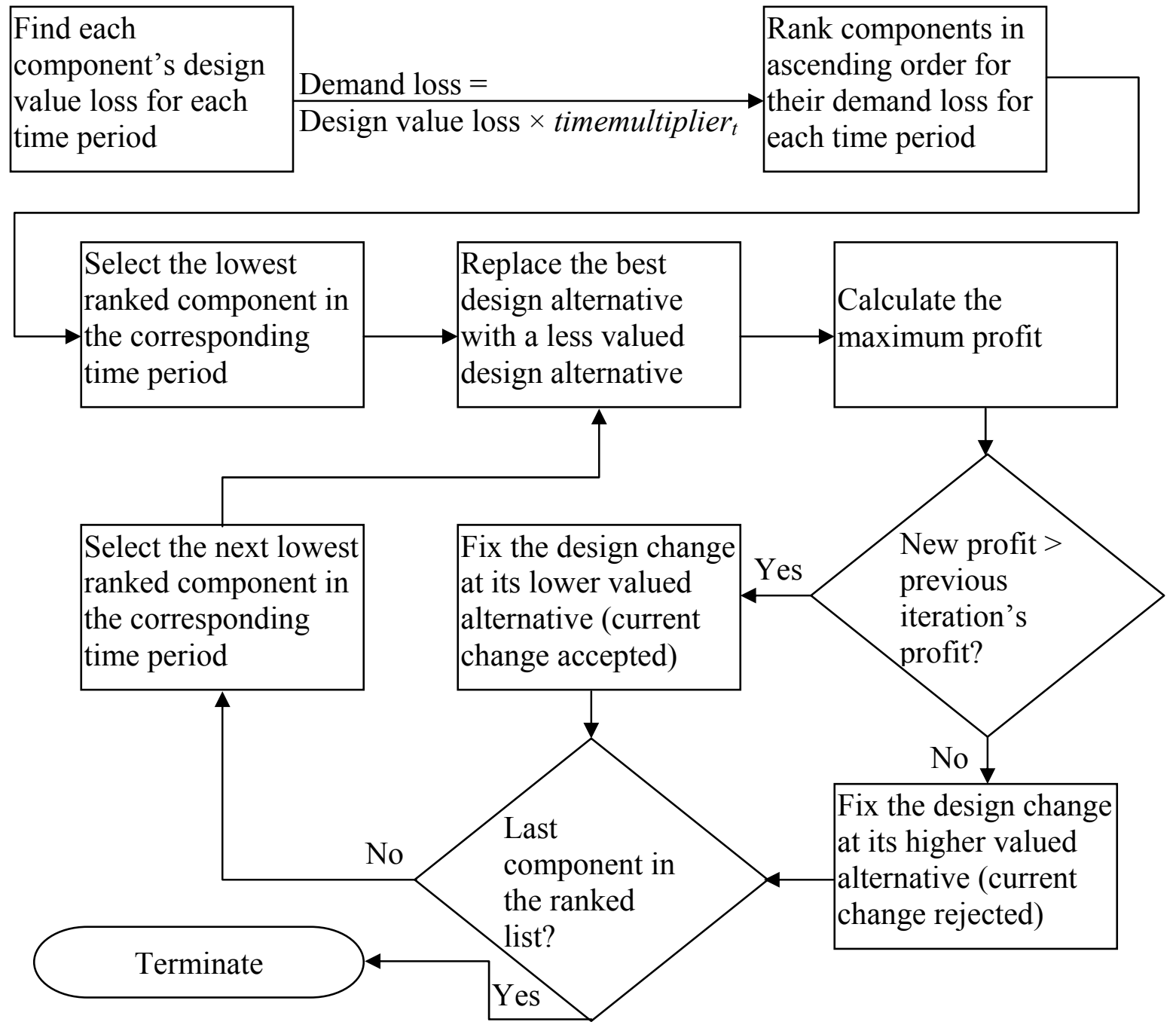

Figure 23: The sequential approach procedure based on separate time periods

Moving through this procedure, several iterations are tested until no improvements on the total profit is recorded. The simultaneous and sequential approaches are compared by first solving the cordless phone example. For this example, the four time multipliers corresponding to four different product life cycle phases are 1, 2, 3, and 1; respectively. Table 26 shows the result of the simultaneous approach and summarizes the results of the sequential approach. 
In the first scenario, all components' first designs are selected (best product design possible). In each following scenario, the component design with the lowest impact on the demand is changed to the second design alternative. The demand loss column shows the impact of this design change on the demand. If the profit is increased with this design change, then this change is accepted and subsequent scenarios include this change. If the change does not increase the profit, then it is rejected and is not investigated in the subsequent scenarios.

Table 26: Simultaneous and sequential approach results for the cordless phone example

\begin{tabular}{clcccc}
\hline Scenario & \multicolumn{1}{c}{ Component } & $\begin{array}{c}\text { Time } \\
\text { Period }\end{array}$ & $\begin{array}{c}\text { Demand } \\
\text { Loss }\end{array}$ & Profit (\$) & $\begin{array}{c}\text { Design Change } \\
\text { Accepted }\end{array}$ \\
\hline Simultaneous Approach & & & $85,462,662$ & \\
\hline Sequential Approach & & & & \\
1 & - & - & - & $77,830,520$ & Yes \\
2 & Antenna & 1 & $3.4 \%$ & $78,333,287$ & Yes \\
3 & Antenna & 4 & $3.4 \%$ & $78,621,416$ & Yes \\
4 & Printed Circuit Board & 1 & $3.9 \%$ & $78,951,870$ & Yes \\
5 & Battery & 1 & $5.1 \%$ & $79,567,470$ & Yes \\
6 & Housing & 1 & $6.4 \%$ & $79,626,229$ & Yes \\
7 & Antenna & 2 & $6.8 \%$ & $82,127,598$ & No \\
8 & Printed Circuit Board & 4 & $7.2 \%$ & $75,996,840$ & Yes \\
9 & Printed Circuit Board & 2 & $10.0 \%$ & $82,878,307$ & Yes \\
10 & Antenna & 3 & $10.2 \%$ & $85,462,662$ & No \\
11 & Housing & 4 & $13.2 \%$ & $83,101,544$ & No \\
12 & Housing & 2 & $13.6 \%$ & $83,856,203$ & No \\
13 & Printed Circuit Board & 3 & $15.0 \%$ & $79,245,215$ & No \\
14 & Battery & 2 & $15.2 \%$ & $85,032,936$ & No \\
15 & Battery & 4 & $17.6 \%$ & $83,178,938$ & No \\
16 & Housing & 3 & $29.4 \%$ & $83,048,751$ & No \\
17 & Battery & 3 & $37.8 \%$ & $82,370,043$ & \\
\hline
\end{tabular}

The sequential approach test results show that scenarios 2 through 10 (except scenario 8) result in higher profit. Further design changes (scenarios 11 through 17) do not improve the profit from the $\$ 85,462,662$ level obtained in scenario 10 . In this problem, scenario 10 of the sequential 
approach finds the same profit as the simultaneous approach. In addition, the optimal solutions of both approaches are the same, that is, they not only result in the same product design, but also they select the same price levels and the same suppliers. In the second comparison of simultaneous and sequential approaches, the desktop computer problem is solved by following the same procedure described for the cordless phone example. For this example, the time multipliers are $1,1.2,1.6$, and 1 ; respectively for each product life cycle phase. Table 27 shows the results of both design approaches.

Table 27: Simultaneous and sequential approach results for the desktop computer example

\begin{tabular}{clcccc}
\hline Scenario & \multicolumn{1}{c}{ Component } & $\begin{array}{c}\text { Time } \\
\text { Period }\end{array}$ & $\begin{array}{c}\text { Demand } \\
\text { Loss }\end{array}$ & Profit (\$) & $\begin{array}{c}\text { Design Change } \\
\text { Accepted }\end{array}$ \\
\hline Simultaneous Approach & & & $8,495,076,500$ & \\
\hline \multicolumn{2}{c}{ Sequential Approach } & & & & \\
1 & - & - & - & $7,561,769,600$ & No \\
2 & Hard Disk & 1 & $3.0 \%$ & $7,539,925,100$ & Yes \\
3 & Hard Disk & 2 & $3.6 \%$ & $7,669,845,600$ & No \\
4 & Peripherals & 1 & $4.0 \%$ & $7,648,320,500$ & No \\
5 & Memory & 1 & $4.0 \%$ & $7,647,961,500$ & Yes \\
6 & Peripherals & 2 & $4.8 \%$ & $7,795,946,900$ & No \\
7 & Monitor & 1 & $6.0 \%$ & $7,747,072,600$ & Yes \\
8 & Motherboard \& CPU & 1 & $6.0 \%$ & $7,761,299,800$ & Yes \\
9 & Memory & 2 & $7.2 \%$ & $7,798,211,400$ & No \\
10 & Monitor & 2 & $8.4 \%$ & $7,815,327,500$ & No \\
11 & Hard Disk & 3 & $9.6 \%$ & $7,313,069,500$ & No \\
12 & Peripherals & 4 & $11.0 \%$ & $7,669,300,400$ & No \\
13 & Hard Disk & 4 & $11.0 \%$ & $7,425,286,000$ & No \\
14 & Memory & 4 & $11.0 \%$ & $7,659,417,200$ & No \\
15 & Peripherals & 3 & $12.8 \%$ & $7,658,090,700$ & No \\
16 & Memory & 3 & $12.8 \%$ & $7,679,607,300$ & No \\
17 & Motherboard \& CPU & 2 & $14.4 \%$ & $7,727,076,000$ & No \\
18 & Monitor & 4 & $15.0 \%$ & $7,612,294,200$ & No \\
19 & Monitor & 3 & $19.2 \%$ & $7,581,860,000$ & \\
20 & Motherboard \& CPU & 4 & $28.0 \%$ & $7,413,139,300$ & \\
21 & Motherboard \& CPU & 3 & $28.8 \%$ & $7,548,458,900$ & \\
\hline
\end{tabular}


The results show that the sequential approach based on the previously described procedure cannot find as high a profit value as the simultaneous approach for this problem example. Only four out of the first ten scenarios could improve the profit value where none of the remaining eleven scenarios could find a better objective value. Since the previously described sequential approach procedure performs poorly, another procedure is also tested to represent the sequential approach for this problem. Within this new procedure, the component with the lowest impact on the demand is selected in a similar manner. However, instead of fixing its design selection at a given time period, the problem is solved by releasing this component's design selections for all time periods and fixing other components at their best designs. For example, in the first scenario of this procedure, the lowest total design loss over four time periods is for the hard disk component. Therefore, all other components designs are fixed at their best; the design selections for the hard disk are decided by the optimization process. If a higher profit is noted for this problem, then these design decisions are fixed and the subsequent component's design selections are released. The new modified sequential approach procedure is depicted in Figure 24. Moving through this procedure the sequential approach is tested and the results are shown in Table 28. 
Find each component's total design value loss over all time periods

$\underset{\Sigma_{\mathrm{t}}\left(\text { Design value loss } \times \text { timemultiplier }_{t}\right)}{\longrightarrow} \begin{aligned} & \text { Rank components in } \\ & \text { ascending order for } \\ & \text { their total demand } \\ & \text { loss }\end{aligned}$

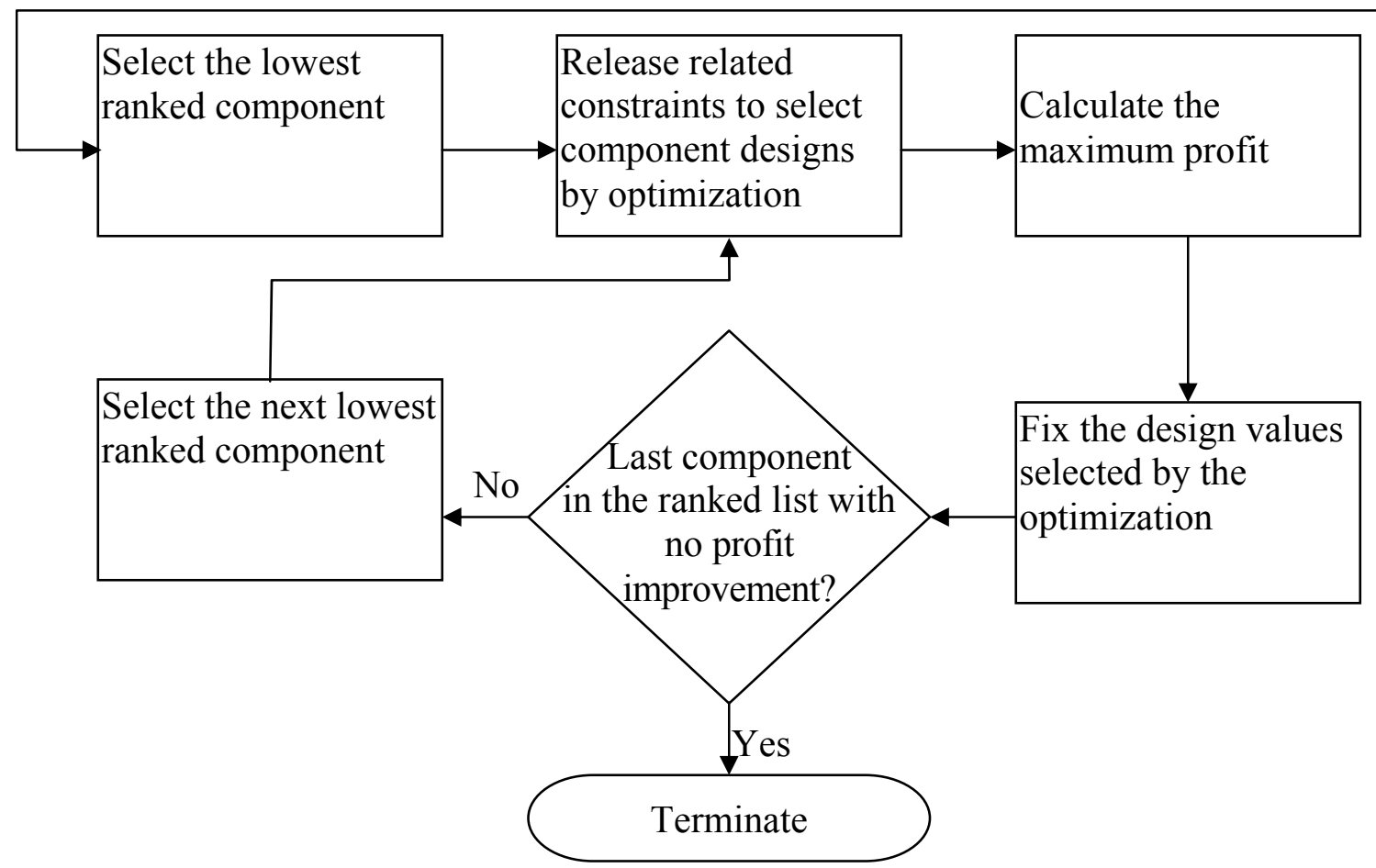

Figure 24: The sequential approach procedure based on the optimization over all time periods 
Table 28: Simultaneous and sequential approach results with alternative sequential procedure

\begin{tabular}{clccc}
\hline Scenario & Component & Total Demand Loss & Profit & $\begin{array}{c}\text { Design Change } \\
\text { Accepted }\end{array}$ \\
\hline Simultaneous Approach & & $8,495,076,500$ & \\
\hline \multicolumn{2}{c}{ Sequential Approach } & & & \\
1 & - & - & $7,561,769,600$ & - \\
2 & Hard Disk & $27.2 \%$ & $7,669,845,000$ & Yes \\
3 & Peripherals & $32.6 \%$ & $7,795,946,900$ & Yes \\
4 & Memory & $35.0 \%$ & $7,798,211,400$ & Yes \\
5 & Monitor & $48.6 \%$ & $7,815,327,500$ & Yes \\
6 & Motherboard \& CPU & $77.2 \%$ & $7,815,327,500$ & No \\
7 & Hard Disk & $27.2 \%$ & $7,815,327,500$ & No \\
8 & Peripherals & $32.6 \%$ & $7,815,327,500$ & No \\
9 & Memory & $35.0 \%$ & $7,917,199,000$ & Yes \\
10 & Monitor & $48.6 \%$ & $7,917,199,000$ & No \\
11 & Motherboard \& CPU & $77.2 \%$ & $7,917,199,000$ & No \\
12 & Hard Disk & $27.2 \%$ & $7,917,199,000$ & No \\
13 & Peripherals & $32.6 \%$ & $7,917,199,000$ & No \\
\hline
\end{tabular}

This change in the sequential procedure eventually finds a better profit value, yet it cannot find the true optimal solution achieved by the simultaneous approach. By letting the optimization process select the component designs, this procedure terminates in fewer iterations. Scenarios 2 through 6 initially consider each component once (Hard Disk, Peripherals, Memory, Monitor, and Motherboard \& CPU). After all components are tested separately, their best design selections are fixed and the iterations are repeated as shown in scenarios 7 through 11. Since an improvement is noted in scenario 9, the two previously rejected components are tested again in scenarios 12 and 13. This procedure makes sure that given a component design selections set, no additional improvements are possible by further changing any component designs.

Based on the presented test results, it is clear that the sequential approach would require many iterations to achieve the same optimal result of the simultaneous approach. However, as the second example shows, the sequential approach may not even be able to find the true optimal 
solution and be stuck with a worse solution. When the optimal prices and component design selections are analyzed for both approaches (shown in Table 29), although there are only minor component design and price differences the profit for the simultaneous approach is higher. Nevertheless, all of these changes that constitute the optimal solution cannot always be determined easily by the sequential approach. The iterative procedure, which the sequential approach utilizes, may miss some alternatives as the interactions between different variables make it hard to capture the profit improvements via an iterative approach. This example illustrates the benefits of the simultaneous approach where all decisions are evaluated in a combined structure, and better solutions are found compared to iteratively trying to improve the solutions.

Table 29: Optimal solutions of simultaneous and sequential approaches - desktop computer

\begin{tabular}{|c|c|c|c|c|c|}
\hline \multirow[t]{2}{*}{ Component } & \multicolumn{4}{|c|}{ Design Selections } & \multirow[t]{2}{*}{ Profit } \\
\hline & $\begin{array}{c}\text { Time } \\
\text { Period } 1\end{array}$ & $\begin{array}{c}\text { Time } \\
\text { Period } 2\end{array}$ & $\begin{array}{c}\text { Time } \\
\text { Period } 3\end{array}$ & $\begin{array}{c}\text { Time } \\
\text { Period } 4\end{array}$ & \\
\hline Simultaneous Approach & & & & & $8,495,076,500$ \\
\hline Motherboard \& CPU & 2 & 1 & 1 & 1 & \\
\hline Memory & 2 & 1 & 1 & 1 & \\
\hline Hard Disk & 2 & 2 & 1 & 1 & \\
\hline Monitor & 2 & 2 & 1 & 1 & \\
\hline Peripherals & 2 & 2 & 1 & 1 & \\
\hline Price & 400 & 400 & 400 & 400 & \\
\hline Sequential Approach & & & & & $7,917,199,000$ \\
\hline Motherboard \& CPU & 1 & 1 & 1 & 1 & \\
\hline Memory & 1 & 1 & 1 & 1 & \\
\hline Hard Disk & 1 & 2 & 1 & 1 & \\
\hline Monitor & 1 & 2 & 1 & 1 & \\
\hline Peripherals & 1 & 2 & 1 & 1 & \\
\hline Price & 800 & 400 & 400 & 400 & \\
\hline
\end{tabular}


In order to further investigate the benefits of using the DFSC and the simultaneous approach, the results of the simultaneous approach, sequential approach, and not using either of these methods (only selecting the best product design and then optimizing the supply chain) are compared by using both examples. Table 30 and Table 31 show the profit, revenue, total and detailed supply chain costs, and final product's lead time values for these three methods for the cordless phone and desktop computer examples, respectively.

Table 30: Revenue, costs, and lead time results of the cordless phone example

\begin{tabular}{lccc}
\hline & $\begin{array}{c}\text { Simultaneous } \\
\text { Approach }\end{array}$ & $\begin{array}{c}\text { Sequential } \\
\text { Approach }\end{array}$ & $\begin{array}{c}\text { Best Product } \\
\text { Design }\end{array}$ \\
\hline Profit $(\$)$ & $85,462,662$ & $85,462,662$ & $77,830,520$ \\
Revenue $(\$)$ & $156,014,344$ & $156,014,344$ & $168,000,000$ \\
Total Supply Chain Cost $(\$)$ & $70,551,682$ & $70,551,682$ & $90,169,479$ \\
Manufacturing Cost $(\$)$ & $26,444,006$ & $26,444,006$ & $32,052,857$ \\
Supply Chain Network Cost $(\$)$ & $15,070,000$ & $15,070,000$ & $15,070,000$ \\
Transportation Cost $(\$)$ & $11,993,713$ & $11,993,713$ & $16,193,142$ \\
Inventory Cost $(\$)$ & $17,043,963$ & $17,043,963$ & $26,853,480$ \\
Lead Time* (days) & $5-10-19-9$ & $5-10-19-9$ & $11-18-23-11$ \\
\hline *Four different values in the table
\end{tabular}

${ }^{*}$ Four different values in the table correspond to final product lead times in four time periods.

Table 31: Revenue, costs, and lead time results of the desktop computer example

\begin{tabular}{lccc}
\hline & $\begin{array}{c}\text { Simultaneous } \\
\text { Approach }\end{array}$ & $\begin{array}{c}\text { Sequential } \\
\text { Approach }\end{array}$ & $\begin{array}{c}\text { Best Product } \\
\text { Design }\end{array}$ \\
\hline Profit $(\$)$ & $8,495,076,500$ & $7,917,199,000$ & $7,561,769,600$ \\
Revenue $(\$)$ & $13,945,540,000$ & $12,850,180,000$ & $12,940,800,000$ \\
Total Supply Chain Cost $(\$)$ & $5,450,459,560$ & $4,932,977,064$ & $5,379,030,446$ \\
Manufacturing Cost $(\$)$ & $4,351,615,400$ & $3,936,897,800$ & $4,273,806,900$ \\
Supply Chain Network Cost $(\$)$ & $17,480,000$ & $17,480,000$ & $17,480,000$ \\
Transportation Cost $(\$)$ & $693,433,690$ & $634,276,890$ & $703,595,610$ \\
Inventory Cost $(\$)$ & $387,930,470$ & $344,322,374$ & $384,147,936$ \\
Lead Time (days) & $5-10-9-9$ & $7-10-9-9$ & $7-11-9-9$ \\
\hline${ }^{*}$ Four different values in the table correspond to final product lead times in four time periods.
\end{tabular}

*Four different values in the table correspond to final product lead times in four time periods. 
As stated before, both the simultaneous and the sequential approaches find the same optimal solution for the cordless phone example. When these results are compared to the best product design selection scenario, it is shown that these methods find a higher profit. Although the best product design approach generates more demand and thus more revenue, both the simultaneous and the sequential approaches find less costly supply chains and therefore have higher profits. It is also shown that for this example, selecting the best product design would yield higher lead times compared to the other two approaches, increasing the inventory costs significantly.

For the desktop computer example, the simultaneous approach finds the best solution, followed by the sequential approach, and then by select the best product design scenario. Changes in the price decisions impact the revenues for these approaches. The simultaneous approach can satisfy more demand by better supplier selection at a lower price as compared to the select the best product design scenario. Although more demand is created in the select the best product design scenario, this high demand cannot be completely satisfied resulting in lower revenue. The sequential approach has the lowest revenue since it cannot generate more demand than the best product design scenario nor supports a lower price and higher demand satisfaction as in the simultaneous approach. The results show that the simultaneous approach creates a more costly supply chain (total supply chain cost in Table 31 ) in addition to its higher revenue however it finds the maximum overall profit. For example, it finds a supply chain with shortest lead times, but its higher demand causes higher inventory costs compared to other two approaches.

In summary, the test results show that selecting the best component design alternatives does not necessarily provide the maximum profit although it satisfies the customer's needs at the maximum level. The test results illustrate that selecting less valued component design 
alternatives (via simultaneous or sequential method) may improve the performance and the profits of the product throughout its life cycle. However, when one of these methods is selected for improving the product design, the results indicate that the simultaneous approach finds better solutions than the sequential approach and the sequential approach may not be able to achieve the same solution. An additional benefit of using the simultaneous approach is that once it finds the optimal solution, no further iterations are required. On the other hand for the sequential approach, many iterations may be required where each time large optimization problems are solved. Yet these iterations may not even improve the profit and the sequential approach may terminate with a worse result.

The results suggest that the simultaneous approach does not necessarily reduce the total supply chain costs or the product lead time, but it balances the cost with the revenue to find a higher profit. Therefore, it helps to overcome the complex interactions between the revenue and cost sides of the profit which is hard to analyze separately in a sequential approach. The simultaneous approach also modifies the product design to achieve better performance in such a way that the other approaches cannot do iteratively. The simultaneous approach may compromise some customer satisfaction, however it accounts for the tradeoff of customer satisfaction with a better supply chain performance.

\subsubsection{Combining Product and Supply Chain Design}

\section{Research Question 3: How robust is the supply chain to product design changes?}

The third research question investigates the robustness of the supply chain with respect to the product design changes. In order to test the impacts of the product design on the supply chain, both of the previously described cordless phone and the desktop computer examples' 
resulting supply chains are analyzed. Table 32 shows the design selections and resulting supply chains for the simultaneous approach and the best design approach for the cordless phone example.

Table 32: Product design and supply chain decisions for the cordless phone example

\begin{tabular}{|c|c|c|c|c|c|c|c|c|}
\hline \multirow[t]{2}{*}{ Component } & \multicolumn{4}{|c|}{ Design Selections } & \multicolumn{4}{|c|}{ Selected Suppliers } \\
\hline & $\begin{array}{l}\text { Time } \\
\text { Per. } 1\end{array}$ & $\begin{array}{l}\text { Time } \\
\text { Per. } 2\end{array}$ & $\begin{array}{l}\text { Time } \\
\text { Per. } 3\end{array}$ & $\begin{array}{l}\text { Time } \\
\text { Per. } 4 \\
\end{array}$ & $\begin{array}{l}\text { Time } \\
\text { Per. } 1 \\
\end{array}$ & $\begin{array}{l}\text { Time } \\
\text { Per. } 2\end{array}$ & $\begin{array}{l}\text { Time } \\
\text { Per. } 3 \\
\end{array}$ & $\begin{array}{l}\text { Time } \\
\text { Per. } 4 \\
\end{array}$ \\
\hline \multicolumn{9}{|l|}{$\begin{array}{l}\text { Simultaneous } \\
\text { Approach }\end{array}$} \\
\hline Housing & 2 & 1 & 1 & 1 & $3-4$ & $1-3$ & $1-3$ & $1-3$ \\
\hline Battery & 2 & 1 & 1 & 1 & 5 & $4-5$ & $4-5$ & 5 \\
\hline Printed Circuit Board & 2 & 2 & 1 & 1 & 2 & 2 & 2 & 2 \\
\hline Antenna & 2 & 2 & 2 & 2 & 5 & 5 & 5 & 5 \\
\hline Price & 40 & 40 & 40 & 40 & & & & \\
\hline \multicolumn{9}{|l|}{$\begin{array}{l}\text { Best Design } \\
\text { Approach }\end{array}$} \\
\hline Housing & 1 & 1 & 1 & 1 & 1 & $1-3$ & $1-3$ & 1 \\
\hline Battery & 1 & 1 & 1 & 1 & $4-5$ & 5 & $4-5$ & $4-5$ \\
\hline Printed Circuit Board & 1 & 1 & 1 & 1 & 2 & 2 & 2 & 2 \\
\hline Antenna & 1 & 1 & 1 & 1 & 5 & 5 & 5 & 5 \\
\hline Price & 40 & 40 & 40 & 40 & & & & \\
\hline
\end{tabular}

The cordless phone example results show some changes in the supplier selection over time for different component design selections. For the housing and battery components, some suppliers are selected or unselected in the optimal solution depending on the demand and capacity limitations. However, for the printed circuit board and antenna components, the same suppliers are selected over all time periods although different designs were chosen for these components. Table 33 presents the design selections and the supply chain alternatives for the desktop computer example. 
Table 33: Product design and supply chain decisions for the desktop computer example

\begin{tabular}{lcccccccc}
\hline \multicolumn{1}{c}{ Component } & \multicolumn{3}{c}{ Design Selections } & \multicolumn{5}{c}{ Selected Suppliers } \\
\cline { 2 - 9 } & \multicolumn{1}{c}{ Time } & Time & Time & Time & Time & Time & Time & Time \\
& Per. 1 & Per. 2 & Per. 3 & Per. 4 & Per. 1 & Per. 2 & Per. 3 & Per. 4 \\
\hline Simultaneous & & & & & & & & \\
Approach & & & & & & & & \\
\hline Motherboard \& CPU & 2 & 1 & 1 & 1 & $2-3-7$ & $2-3-7$ & $2-3-7$ & $2-7$ \\
Memory & 2 & 1 & 1 & 1 & $2-5$ & $1-2-5$ & $1-2-5$ & $2-5$ \\
Hard Disk & 2 & 2 & 1 & 1 & 6 & 6 & $3-5-6$ & $3-6$ \\
Monitor & 2 & 2 & 1 & 1 & 3 & 3 & $3-4-5$ & $3-5$ \\
Peripherals & 2 & 2 & 1 & 1 & 7 & 7 & $2-6-7$ & $6-7$ \\
Price & 400 & 400 & 400 & 400 & & & & \\
\hline Best Design & & & & & & & & \\
Approach & 1 & 1 & 1 & 1 & $2-3$ & $2-3-7$ & $2-3-7$ & $2-7$ \\
\hline Motherboard \& CPU & 1 & 1 & 1 & 1 & $2-5$ & $1-2-5$ & $1-2-5$ & $2-5$ \\
Memory & 1 & 1 & 1 & 1 & 6 & $3-5-6$ & $3-5-6$ & $3-5$ \\
Hard Disk & 1 & 1 & 1 & 1 & 5 & $3-4-5$ & $3-4-5$ & $3-5$ \\
Monitor & 1 & 1 & 1 & 1 & 7 & $6-7$ & $2-6-7$ & $6-7$ \\
Peripherals & 800 & 400 & 400 & 400 & & & & \\
Price & & & & & & & &
\end{tabular}

The desktop computer example results show that the simultaneous approach selected different suppliers for the hard disk and monitor components over time. The change in the supply chain is a result of different demand due to design selections and related supplier capacities. It should also be noted that although it is not explicitly modeled and implied in the objective function, the simultaneous approach results generate a supply chain with fewer suppliers, which would reduce the supply chain complexity in real industry applications.

As these example problems illustrate, different component design selections would necessitate different supply chain designs as changing demand and price values would require use of more supplier capacity or a less costly supplier in the optimal solution. These requirements may necessitate new suppliers to be added to or some existing suppliers to be removed from the supply chain as well as replacement of some suppliers by others to 
accommodate new cost or capacity requirements. Although these examples do not explicitly indicate any change in the supply chain tiers, real industry problems may require more components to be added into the product bill of material depending on the component design selections which may further result in new supply chain tiers.

\section{Research Question 4: What is the relative importance of the product design and the supply chain design on the product success (thus on the profits)?}

The fourth research question investigates whether the product design or the supply chain dominates each other's impact on the product's success and profits. The two examples' test results indicate a strong interdependence between the product design and the supply chain, and that they both have significant impacts on the product's profits. As shown in Table 30 for the cordless phone example, sometimes it may be preferred to reduce the demand by selecting less attractive component designs in order to reduce the supply chain costs and to maximize the profit. However, due to strong interdependence, as shown in Table 31 for the desktop computer example, it might be better to increase the demand by selecting better product designs so that more revenue is generated although supply chain costs are increased. Therefore, the results indicate that neither product design nor the supply chain dominate each other in terms of significance of their impacts on the profit generation. This result stresses the importance of the simultaneous approach as it aims to balance these two important aspects to maximize the profit where overlooking either one (as shown in best product design approach results) may lead to non-optimal results. 


\subsection{CONCLUSIONS AND FUTURE RESEARCH}

This chapter highlights the findings of this research and summarizes the conclusions in the first section. In the second section, further concepts, ideas, and opportunities which can be added to extend the developed DFSC models are presented. This section also discusses further enhancements to the presented solution techniques and alternative solution procedures.

\subsection{SUMMARY AND CONCLUSIONS}

In this dissertation, an optimization model which aims to maximize the profit of a product throughout its life cycle by deciding its components' design alternatives, the associated supply chain, and price levels is presented. This DFSC model provides an opportunity to simultaneously consider product design and supply chain decisions within the product design phase which would replace the sequential approach that is commonly used in industry. The simultaneous approach helps the decision makers to see the big picture and impacts of their decisions both in the short and long term. It also provides an alternative approach for optimizing these decisions to achieve better results which cannot always be accomplished by the sequential approach. Finally, the simultaneous approach generally works faster than the sequential approach which may require multiple iterations, thus it helps to finalize the product design and supply chain decisions earlier, reducing product design time and product time-to-market. 
The test results suggest that both the product design and the supply chain are important decisions and both have significant impacts on a product's profitability. Therefore, emphasis must be placed on both aspects and neither one should be overlooked. The presented simultaneous approach offers an analytical framework to achieve this balanced method where both aspects are considered before any major decisions are made as it is very costly to change the product design or supply chain decisions in the later phases of a product's life cycle.

The computational test results further suggest that the DFSC models tend to be very complex in nature due to very complicated relationships and interdependencies between product design and supply chain variables. These models grow significantly in size as the number of product components and the number of suppliers increase. According to the test results and industry experts' suggestions, the developed reduced DFSC models are attractive alternatives where the full-scale complete DFSC models cannot be solved in reasonable times.

In terms of solution methodologies, the test results show that metaheuristics can be utilized to improve the quality of the solutions for the DFSC models where they cannot be solved easily by the deterministic optimization methodologies, such as the MIP. However, the test results also show that the performance of both solution methodologies depends both on the problem size and the complexity of the interactions between the problem parameters. Since the MIP guarantees an optimal solution, it is the preferred solution methodology. However, when the solution time becomes unreasonable due to the problem size and complexity, metaheuristics (GA) become a good solution alternative.

According to the discussions with the industry experts and their model reviews, the developed DFSC models would add important value to their product design processes and would be a very useful decision support tool. The industry experts suggest that although further 
extensions and customization of the DFSC models would increase their value, the developed models are valid and show the underlying benefits of the simultaneous product and supply chain design optimization approach. In terms of model applicability, the industry experts agree that the DFSC model has diverse data requirements, yet this data is customarily gathered. Hence, the DFSC model can be easily implemented and utilized with the data in hand.

This research provides a significant addition to the existing DFSC research by combining the product design and supply chain decisions into a single framework which optimizes the decisions simultaneously. This research aims to fill in the gap in the DFSC literature which lacks explicit consideration and integration of marketing and product design decisions and manufacturing and supply chain decisions. Both the demand and manufacturing aspects of a product are addressed uniquely in this research and well-established product design and supply chain performance metrics are employed. The generic DFSC models which are applicable to virtually any type of product in any manufacturing industry are solved by utilizing Mixed Integer Programming and Genetic Algorithm. Detailed computational tests and illustrative examples provide a clear assessment of both solution methodologies. In addition, this dissertation evaluates alternative modeling preferences and assesses the performance of different solution methodologies for these alternative models. Furthermore, the presented analyses provide insight into the modeling and algorithmic / computation complexity issues.

Finally, this dissertation provides an important assessment from different industry experts across various industries and includes these experts' validation and suggestions for further work. 


\subsection{EXTENSIONS FOR DFSC MODELS AND SOLUTION METHODS}

The developed DFSC model aims to capture important decision variables which a product design or supply chain expert would consider for a new product. However, it is certain that some particular extensions of this model and further customizations would improve the quality of the model and help it fit different products' unique requirements. In this section, some additional objectives, constraints, and concepts are presented.

Although a company's main objective is usually profit maximization, some other objectives might be pursued occasionally in order to achieve other goals. For example, when the company wishes to penetrate into a new market with a new product design, its main objective might be demand or market share maximization rather than maximization of profit. Although, the main objective in the long term would still be profit maximization, this short term objective to maximize demand may overrule the profit maximization objective and the company may even allow a short term loss. Therefore, an important extension for the DFSC model would be introduction of different objectives for different time periods to reflect changing priorities of a company for a product. Introduction of different objectives for different time periods should not be confused with a multi-objective formulation since these various objectives here are aimed to be unique goals which vary in different time periods. In addition to the market share maximization objective, some other important objectives include maximizing the reliability of the supply chain and reducing the negative impacts of the product design and supply chain on the environment. The maximization of the supply chain reliability is an important issue since variations in supplier quality and lead times have significant impacts on the supply chain performance and profitability in the later phases of the product life cycle. In addition, the product

design influences the environment by dictating material selection and certain manufacturing 
processes. For example, using a plastic component may have a more negative impact on the environment than using a metal component if the product is not recycled properly at the end of its life cycle. A product design may also require certain manufacturing processes that have harmful effects on the environment such as generating chemical waste. Therefore, minimizing the harmful impacts of the product and supply chain design on the environment could be a good extension for the DFSC models.

In addition to the changes in the objective function, the DFSC model can be extended by adding or changing some constraints to capture other important concepts or requirements. For example, as the industry experts stated, many companies make their supplier selections not only by using the quantitative variables (such as cost and lead time) but they also consider some qualitative metrics or constraints. The industry experts listed quality levels of suppliers, risks and opportunities of a supplier, and previous business history with some suppliers as important qualitative metrics. However, it would be beneficial to quantify these metrics by using a structured methodology (such as Analytical Hierarchy Process) or using a surrogate measure such as number of defective parts per million for the quality before they are introduced into the DFSC model.

In terms of other quantitative constraints, the DFSC models can be improved by adding specific supply chain network constraints that many companies consider. For example, these constraints may include the requirement of using at least two distinct suppliers for the critical parts, some minimum order quantities for certain suppliers, or some other constraints that are related to outsourcing decisions and protection of intellectual property. For instance, it would be an interesting extension to study the impact of different component design selections on the 
protection of the intellectual property where some design alternatives cannot be manufactured by the main company or by the domestic suppliers.

As today's business environment involves complex decisions for outsourcing to overseas and offshoring, the addition of related variables such as custom duty rates would also add significant value to the DFSC models. Since these decisions often need to take the import and export related issues and costs into account, the DFSC can easily capture these important aspects. Some important variables identified by the industry experts include custom duty rates, taxation variations in different countries, import / export quotas, additional transportation challenges such as vessel capacity problems, and other risks related to other countries' political and economical circumstances. Some of these variables such as quotas and taxes are quantitative measures and can be added into the DFSC models with relative ease. Yet, other variables such as economic or political risks are qualitative in nature and require further assessment and need to be analytically quantified for integration into the DFSC models. Expansion of the DFSC models to incorporate these outsourcing and offshoring variables is important for global supply chain decisions and would add value to the DFSC models.

Although the deterministic approach employed in this dissertation captures and estimates the impacts of the most important supply chain performance criteria, the explicit modeling of the stochastic elements would be another important extension. The demand and supply chain lead time parameters (which are approximated by assuming constant coefficients of variation in this dissertation) can be further analyzed by a stochastic modeling approach. The impacts of the demand and the lead time on the profits can be investigated in detail through a simulation analysis integrated within the optimization procedure. However, the additional computational 
requirements associated with the simulation should be carefully weighed against the model improvements. 


\section{APPENDIX A}

\section{MODEL LINEARIZATION SCHEMA}

In this section, the linearization schemas that are developed to remove nonlinearities from the mathematical model are described.

\section{A.1 REVENUE LINEARIZATION}

In order to represent the Total Revenue with linear equations, initially two sets of variables $\left(\tau_{t}^{+}, \tau_{t}^{-}\right.$and $\left.k_{t}^{+}, k_{t}^{-}\right)$are introduced. As explained in their definition in section 3.3 , these variables are used to evaluate satisfied demand which is

$$
\text { SatisfiedDemand }_{t}=\min \left(\text { Demand }_{1 t} \text {, Total Production }{ }_{1 t}\right)
$$

Based on their definitions, these variables take respective values as shown below.

$$
\begin{aligned}
& \text { If Demand }{ }_{1 t}>\sum_{\alpha_{1}=1}^{A_{1}} \sum_{j=1}^{S}\left(x_{1 j \alpha_{1} t}^{1}+x_{1 j \alpha_{1} t}^{2}\right), \text { then }\left\{\begin{array}{l}
\tau_{t}^{+}=0 \Rightarrow k_{t}^{+}=0 \\
\tau_{t}^{-}=1 \Rightarrow k_{t}^{-}=\sum_{\alpha_{1}=1}^{A_{1}} \sum_{j=1}^{S}\left(x_{1 j \alpha_{1} t}^{1}+x_{1 j \alpha_{1} t}^{2}\right)
\end{array}\right. \\
& \text { If } \sum_{\alpha_{1}=1}^{A_{1}} \sum_{j=1}^{S}\left(x_{1 j \alpha_{1} t}^{1}+x_{1 j \alpha_{1} t}^{2}\right)>\text { Demand }_{1 t} \text {, then }\left\{\begin{array}{l}
\tau_{t}^{+}=1 \Rightarrow k_{t}^{+}=\text {Demand }_{1 t} \\
\tau_{t}^{-}=0 \Rightarrow k_{t}^{-}=0
\end{array}\right.
\end{aligned}
$$


Since, according to the presented representation, $k_{t}^{+}$and $k_{t}^{-}$would take relevant demand or total production values, revenue in each time period can be expressed as

$$
\text { Revenue }_{t}=\left(\text { price }_{t} \times\left(k_{t}^{+}+k_{t}^{-}\right)\right)
$$

As described in section 3.1, a quadratic price - demand function can be expressed by

$$
\text { Demand }=\beta_{1} \text { price }^{2}+\beta_{2}
$$

By using this function, the demand can be expressed as a function of price, product design value, and time period adjustment parameter by

$$
\text { Demand }_{1 t}=\left(\beta_{1} \text { price }_{t}^{2}+\beta_{2}\right) \times v_{t} \times \text { timemultiplier }_{t}
$$

As the price is allowed to take discrete values $\left(\omega_{1 t}, \omega_{2 t}, \omega_{3 t}\right)$ in this model, the final price value in each time period is expressed by

$$
\text { price }_{t}=\omega_{1 t}+\left(\omega_{2 t}-\omega_{1 t}\right) \phi_{1 t}+\left(\omega_{3 t}-\omega_{1 t}\right) \phi_{2 t}
$$

where, $\phi_{1 t}$ and $\phi_{2 t}$ variables control if price values are increased to $\omega_{2 t}$ and/or $\omega_{3 t}$, respectively. From equations (A.4) and (A.7), it can be concluded that regardless of the price levels, revenue would at least be equal to $\omega_{1 t}\left(k_{t}^{+}+k_{t}^{-}\right)$. Since the demand function is utilized in a separate constraint in the model, a direct mathematical equation of the revenue and demand functions does not exist. Therefore, having $k_{t}^{+}$and $k_{t}^{-}$take relevant demand or total production values via separate constraints given in (6.30) and (6.33), it is not necessary to linearize the revenue function with respect to price - product design value multiplication. However, an additional set of variables $\left(\psi_{1 t}, \psi_{2}, \psi_{3 t}, \psi_{4 t}\right)$ is introduced to represent revenue values for higher price levels. The actual non-linear relations that these variables represent are given below 


$$
\begin{aligned}
& \psi_{1 t}=k_{t}^{+} \times \phi_{1 t} \\
& \psi_{2 t}=k_{t}^{+} \times \phi_{2 t} \\
& \psi_{3 t}=k_{t}^{-} \times \phi_{1 t} \\
& \psi_{4 t}=k_{t}^{-} \times \phi_{2 t}
\end{aligned}
$$

Since constraints given in equations (6.34) through (6.45) force only one of the $\psi$ values to take a non-zero value, the revenue function can finally be expressed by

$$
\text { Revenue }_{t}=\omega_{1 t}\left(k_{t}^{+}+k_{t}^{-}\right)+\left(\omega_{2 t}-\omega_{1 t}\right)\left(\psi_{1 t}+\psi_{3 t}\right)+\left(\omega_{3 t}-\omega_{1 t}\right)\left(\psi_{2 t}+\psi_{4 t}\right)
$$

Accordingly, Total Revenue would be expressed by

$$
\text { Total Revenue }=\sum_{t=1}^{T} \text { Revenue }_{t}=\sum_{t=1}^{T} \omega_{1 t}\left(k_{t}^{+}+k_{t}^{-}\right)+\left(\omega_{2 t}-\omega_{1 t}\right)\left(\psi_{1 t}+\psi_{3 t}\right)+\left(\omega_{3 t}-\omega_{1 t}\right)\left(\psi_{2 t}+\psi_{4 t}\right)
$$

\section{A.2 DEMAND LINEARIZATION}

Since the quadratic price - demand function is given as

$$
\text { Demand }=\beta_{1} \text { price }^{2}+\beta_{2}
$$

and the final price value in each time period is expressed by

$$
\text { price }_{t}=\omega_{1 t}+\left(\omega_{2 t}-\omega_{1 t}\right) \phi_{1 t}+\left(\omega_{3 t}-\omega_{1 t}\right) \phi_{2 t}
$$

the demand function becomes

$$
\text { Demand }_{1 t}=\left(\beta_{1} \omega_{1 t}^{2}\left(1-\phi_{1 t}-\phi_{2 t}\right)+\beta_{1} \omega_{2 t}^{2} \phi_{1 t}+\beta_{1} \omega_{3 t}^{2} \phi_{2 t}+\beta_{2}\right) \times v_{t} \times \text { timemultiplier }_{t}
$$

since $\phi_{1 t} \times \phi_{2 t}=0$ is always true by definition and via equation (6.21). 
In order to represent Demand $_{1 t}$ as a linear equation, an additional set of variables $\left(\lambda_{1 t}, \lambda_{2 t}\right)$ is introduced to linearize price - product design value multiplication. The actual non-linear relations that these variables represent are given below

$$
\begin{aligned}
& \lambda_{1 t}=\phi_{1 t} \times v_{t} \\
& \lambda_{2 t}=\phi_{2 t} \times v_{t}
\end{aligned}
$$

By using the Demand $d_{1 t}$ function (A.13) and replacing $\phi \times v$ multiplications by $\lambda$ variables, the final Demand $_{1 t}$ function becomes

$$
\text { Demand }_{1 t}=\left(\left(\beta_{1} \omega_{1 t}^{2}+\beta_{2}\right) v_{t}+\beta_{1} \lambda_{1 t}\left(\omega_{2 t}^{2}-\omega_{1 t}^{2}\right)+\beta_{1} \lambda_{2 t}\left(\omega_{3 t}^{2}-\omega_{1 t}^{2}\right)\right) \times \text { timemultiplier }_{t}
$$

\section{A.3 INVENTORY COST LINEARIZATION}

Since the developed model is at the strategic planning level, the inventory levels are calculated as the average Safety Stock levels for each time period. Based on the inventory theory and wide application in the literature, the Safety Stock level for a given customer satisfaction target under stochastic demand and lead time is given as the following formula [49].

$$
\text { Safety Stock }=z_{\text {ssratio }} \sqrt{\bar{L} \sigma_{D}^{2}+(\bar{D})^{2} \sigma_{L}^{2}}
$$

where $\bar{L}$ is the average lead time, $\bar{D}$ is the average demand during the average lead time, and $\sigma_{L}^{2}$ and $\sigma_{D}^{2}$ are the variances of the lead time and demand during the lead time, respectively.

The lead time values used in this research $\left(L T_{l t}\right)$ are given in actual true time units. However, the demand values (Demand ${ }_{1 t}$ ) are calculated for the entire product life cycle phase $(t)$ therefore, the average demand during the lead time is calculated by $\frac{\text { Demand }_{1 t}}{\text { periodlength }}$, where 
periodlength $h_{t}$ is the length of the time period $t$ in the same units with lead time. Therefore, by using the parameters and variables of the DFSC model, the Safety Stock in a time period can be represented as

$$
\text { Safety Stock }{ }_{t}=z_{\text {ssatio }} \sqrt{L T_{1 t} \sigma_{\text {Demand }}^{2}+\frac{\text { Demand }_{1 t}^{2}}{\text { periodlength }_{t}{ }^{2}} \sigma_{L T}^{2}}
$$

In this research, it is assumed that lead time and lead time demand have constant coefficients of variation, which suggests that change in the variation of these variables is constant when their average values are changed. The mathematical representations of $\rho_{1}$ and $\rho_{2}$ by using model variables are given below.

$$
\begin{gathered}
\rho_{1}=\frac{\sigma_{\text {Demand }_{\text {d }}}}{\frac{\text { Demand }_{1 t}}{\text { periodlength }_{t}}} \\
\rho_{2}=\frac{\sigma_{L T}}{L T_{1 t}}
\end{gathered}
$$

By using $\rho_{1}$ and $\rho_{2}$ for coefficients of variation for lead time and demand over the lead time respectively, the safety stock in a time period can be represented as

$$
\begin{aligned}
\text { Safety Stock }_{t} & =z_{\text {ssratio }} \sqrt{L T_{1 t} \rho_{1}^{2} \frac{\text { Demand }_{1 t}{ }_{\text {periodlength }}{ }_{t}{ }^{2}}{\text { periodlength }_{t}{ }^{2}} \rho_{2}^{2} L T_{1 t}^{2}} \\
& =z_{\text {ssratio }} \frac{L T_{1 t} \text { Demand }_{1 t}}{\text { periodlength }_{t}} \sqrt{\frac{\rho_{1}^{2}}{L T_{1 t}}+\rho_{2}^{2}}
\end{aligned}
$$

Since the total inventory would be equal to the sum of the average lead time inventory kept and safety stock, the Total Inventory in a time period is represented as

$$
\text { Total Inventory }_{t}=\frac{L T_{1 t} \text { Demand }_{1 t}}{\text { periodlength }_{t}}+z_{\text {ssatio }} \frac{L T_{1 t} \text { Demand }_{1 t}}{\text { periodlength }_{t}} \sqrt{\frac{\rho_{1}^{2}}{L T_{1 t}}+\rho_{2}^{2}}
$$

Since this model calculates the demand at the strategic level, the lead time demand variation is removed by assuming $\rho_{l}=0$. Hence, the Total Inventory in a time period becomes 
Total Inventory $_{t}=\frac{L T_{1 t} \text { Demand }_{1 t}}{\text { periodlength }_{t}}+z_{\text {ssratio }} \frac{L T_{1 t} \text { Demand }_{1 t}}{\text { periodlength }_{t}} \rho_{2}=\frac{L T_{1 t} \text { Demand }_{1 t}}{\text { periodlength }_{t}}\left(1+z_{\text {ssratio }} \rho_{2}\right)$

However, the lead time and demand multiplication makes this equation non-linear therefore a linearization is provided. Since Demand $d_{1 t}$ is a continuous variable, $L T_{1 t}$ is limited to take integer values, given that production time data, $p t_{i j \alpha_{i}}$ is integer and capacity utilization adjustments do not strictly enforce proportional reductions in production times. Furthermore, the lead time values are rounded up to the nearest integer value since it would be better to account for the uncertainty for the worst case scenario. Given these assumptions, lead time values can be represented as combinations of several binary variables $\left(\gamma_{n t}\right)$ as shown in the following equation.

$$
L T_{1 t}=\gamma_{0 t}+2 \gamma_{1 t}+4 \gamma_{2 t}+8 \gamma_{3 t}+16 \gamma_{4 t}+32 \gamma_{5 t}+64 \gamma_{6 t}+\ldots+2^{n} \gamma_{n t}=\sum_{n=0}^{N} 2^{n} \gamma_{n t}, n \in[0, \mathrm{~N}]
$$

It should be noted that based on the provided data, the number of $\gamma$ variables $(N)$ needs to be estimated so that $L T_{I t} \leq 2^{N}$ is always true in order to keep the model feasible. By utilizing this binary representation schema, lead time - demand multiplication becomes

$$
\begin{aligned}
\text { LT }_{1 t} \text { Demand }_{1 t}= & \gamma_{0 t} \text { Demand }_{1 t}+2 \gamma_{1 t} \text { Demand }_{1 t}+4 \gamma_{2 t} \text { Demand }_{1 t}+8 \gamma_{3 t} \text { Demand }_{1 t}+ \\
& 16 \gamma_{4 t} \text { Demand }_{1 t}+32 \gamma_{5 t} \text { Demand }_{1 t}+64 \gamma_{6 t} \text { Demand }_{1 t}+\ldots+ \\
& 2^{n} \gamma_{n t} \text { Demand }_{1 t}
\end{aligned}
$$

By the introduction of $\delta_{n t}$ variables, each of which corresponding to relative $\gamma_{n t}$ Demand $_{1 t}$ multiplications (i.e., $\delta_{n t}=\gamma_{n t}$ Demand $_{1 t}$ for each $n \in[0, \mathrm{~N}]$ ), the lead time - demand multiplication is represented as

$$
\begin{aligned}
L_{1 t} \text { Demand }_{1 t} & =\delta_{0 t}+2 \delta_{1 t}+4 \delta_{2 t}+8 \delta_{3 t}+16 \delta_{4 t}+32 \delta_{5 t}+64 \delta_{6 t}+\ldots+2^{n} \delta_{n t} \\
& =\sum_{n=0}^{N} 2^{n} \delta_{n t}, n \in[0, \mathrm{~N}]
\end{aligned}
$$

In order to calculate Total Inventory Cost, the average inventory in a time period is assumed to be half of the maximum inventory levels. This approach is widely used in the literature and is 
based on the fact that the inventory is used over the lead time thus it is safe to assume that the inventory decreases linearly with the time. Therefore, the average inventory level for a time period can be adequately approximated as the half of the maximum inventory. Based on this assumption, the Total Inventory Cost in a time period can be calculated as

$$
\begin{aligned}
\text { Total Inventory Cost }_{t} & =\frac{1}{2} \text { periodlength }_{t} h_{t} \frac{\text { LT }_{1 t} \text { Demand }_{1 t}}{\text { periodlength }_{t}}\left(1+z_{\text {ssratio }} \rho_{2}\right) \\
& =\frac{1}{2} h_{t} L_{1 t} \text { Demand }_{1 t}\left(1+z_{\text {ssratio }} \rho_{2}\right)
\end{aligned}
$$

By using the lead time - demand linearization (A.25), the linear form of the Total Inventory Cost in a time period, which is used in the objective function, becomes

$$
\text { Total Inventory } \text { Cost }_{t}=\frac{1}{2} h_{t}\left(1+z_{\text {ssratio }} \rho_{2}\right) \sum_{n=0}^{8} 2^{n} \delta_{n t}
$$




\section{APPENDIX B}

\section{REDUCED DFSC MODELS}

In this appendix, the two reduced DFSC model formulations, described in section 3.4, are presented. It should be noted that the same notation used in the complete DFSC modeling is employed.

\section{B.1 REDUCED DFSC MODEL - 1}

The following mathematical model is the reduced DFSC model that reflects the removal of economies of scale concept from the complete model. 


$$
\begin{aligned}
& \max \sum_{t=1}^{T} \omega_{1 t}\left(k_{t}^{+}+k_{t}^{-}\right)+\left(\omega_{2 t}-\omega_{1 t}\right)\left(\psi_{1 t}+\psi_{3 t}\right)+\left(\omega_{3 t}-\omega_{1 t}\right)\left(\psi_{2 t}+\psi_{4 t}\right)-\left(\sum_{t=1}^{T} \sum_{i=1}^{P} \sum_{j=1}^{S} \sum_{\alpha_{i}=1}^{A_{i}} c_{i j \alpha_{i}}^{1} x_{i j \alpha_{i} t}^{1}+\right. \\
& \left.\sum_{j=1}^{S} \sum_{l=1}^{S} y_{j l} \text { Netwco }_{j l}+\sum_{t=1}^{T} \sum_{j=1}^{S} \sum_{k=1}^{P} \sum_{l=1}^{S} \sum_{\alpha_{k}}^{A_{k}} u_{j k l \alpha_{k}} T_{p o r t c o s t}{ }_{j k l \alpha_{k} t}+\sum_{t=1}^{T} \frac{1}{2} h_{t}\left(1+z_{s s r a t i o} \rho_{2}\right) \sum_{n=0}^{N} 2^{n} \delta_{n t}\right)
\end{aligned}
$$

s.t.

Demand $_{1 t}=\left(\left(\beta_{1} \omega_{1 t}^{2}+\beta_{2}\right) v_{t}+\beta_{1} \lambda_{1 t}\left(\omega_{2 t}^{2}-\omega_{1 t}^{2}\right)+\beta_{1} \lambda_{2 t}\left(\omega_{3 t}^{2}-\omega_{1 t}^{2}\right)\right) \times$ timemultip lier $_{t} \forall t$

Demand $_{k t}=\sum_{\mathrm{i}=1}^{\mathrm{k}-1}$ Demand $_{i t}$ Relation $_{i k} \quad \forall k(k>1), t$

$\sum_{\alpha_{k}=1}^{A_{k}} \sum_{j=1}^{S} x_{k j \alpha_{k} t}^{1} \geq \sum_{i=1}^{k-1} \sum_{\alpha_{i}=1}^{A_{i}} \sum_{j=1}^{S} x_{i j \alpha_{i}}^{1}$ Relation $_{i k} \quad \forall k(k>1), t$

$\sum_{j=1}^{S} x_{i j \alpha_{i} t}^{1} \leq \pi_{i \alpha_{i} t} \operatorname{Mcap}_{i \alpha_{i} t}$

$\forall i, \alpha_{i}, t$

$\sum_{\alpha_{i}=1}^{A_{i}} \pi_{i \alpha_{i} t}=1$

$\forall i, t$

$v_{t}=\sum_{i=1}^{P} \sum_{\alpha_{i}=1}^{A_{i}} \pi_{i \alpha_{i}} v a l_{i \alpha_{i} t}$

$\forall t$

$x_{i j \alpha_{i} t}^{1} \leq$ Capacity $_{i j \alpha_{i} t}^{1}$

$\forall i, j, \alpha_{i}, t$

$y_{j l}$ Capacity $_{k l \alpha_{k} t}^{1} \geq u_{j k l \alpha_{k} t}$

$\forall j, k, l, \alpha_{k}, t$

$\sum_{j=1}^{S} u_{j k l \alpha_{k} t} \leq x_{k l \alpha_{k} t}^{1}$

$\forall k, l, \alpha_{k}, t$

$\sum_{\alpha_{k}=1}^{A_{k}} \sum_{l=1}^{S} u_{j k l \alpha_{k} t} \geq$ Relation $_{i k} \sum_{\alpha_{i}=1}^{A_{i}} x_{i j \alpha_{i} t}^{1}$

$\forall i, j, k, t \mid$ Relation $_{i k}>0$

ptime $_{i t} \geq \frac{p t_{i j \alpha_{i}} x_{i j \alpha_{i} t}^{1}}{\text { Capacity }_{i j \alpha_{i}}^{1}}$

$\forall i, j, \alpha_{i}, t \mid$ Capacity $_{i j \alpha t}^{1}>0$

$L T_{i t} \geq L T_{k t}$ Relation $_{i k}+$ ptime $_{i t}$

$\forall i, k, t \mid$ Relation $_{i k}>0$

$L T_{i t} \geq$ ptime $_{i t}$

$\forall i, t \mid$ Relation $_{i k}=0$ for $\forall k>i$

$L T_{1 t}=\sum_{n=0}^{N} 2^{n} \gamma_{n t}$

$\forall t$

$\delta_{n t} \leq$ Demand $_{1 t}$

$n \in[0, \mathrm{~N}], \forall t$

$\delta_{n t} \leq M d e m \times \gamma_{n t}$

$n \in[0, \mathrm{~N}], \forall t$

Demand $_{1 t}-\operatorname{Mdem}\left(1-\gamma_{n t}\right) \leq \delta_{n t}$

$n \in[0, \mathrm{~N}], \forall t$ 


$$
\begin{aligned}
& \phi_{1 t}+\phi_{2 t} \leq 1 \quad \forall t \\
& \lambda_{q t} \leq \phi_{q t} \quad \forall t, q \in\{1,2\} \\
& \lambda_{q t} \leq v_{t} \quad \forall t, q \in\{1,2\} \\
& \phi_{q t}+v_{t} \leq \lambda_{q t}+1 \quad \forall t, q \in\{1,2\} \\
& \tau_{t}^{+} \sum_{\alpha_{1}=1}^{A_{1}} \text { Mcap }_{1 \alpha_{1} t} \geq \sum_{\alpha_{1}=1}^{A_{1}} \sum_{j=1}^{S} x_{1 j \alpha_{1} t}^{1}-\text { Demand }_{1 t} \quad \forall t \\
& \tau_{t}^{-} \text {Mdem } \geq \text { Demand }_{1 t}-\sum_{\alpha_{1}=1}^{A_{1}} \sum_{j=1}^{S} x_{1 j \alpha_{1} t}^{1} \quad \forall t \\
& \tau_{t}^{+}+\tau_{t}^{-} \leq 1 \quad \forall t \\
& k_{t}^{+} \leq \text {Demand }_{1 t \quad} \quad \forall t \\
& k_{t}^{+} \leq \tau_{t}^{+} \text {Mdem } \quad \forall t \\
& \text { Demand }_{1 t}+\tau_{t}^{+} \text {Mdem } \leq k_{t}^{+}+\text {Mdem } \quad \forall t \\
& k_{t}^{-} \leq \sum_{\alpha_{1}=1}^{A_{1}} \sum_{j=1}^{S} x_{1 j \alpha_{1} t}^{1} \\
& k_{t}^{-} \leq \tau_{t}^{-} \sum_{\alpha_{1}=1}^{A_{1}} \operatorname{Mcap}_{1 \alpha_{1} t} \quad \forall t \\
& \sum_{\alpha_{1}=1}^{A_{1}} \sum_{j=1}^{S} x_{1 j \alpha_{1} t}^{1}+\tau_{t}^{-} \sum_{\alpha_{1}=1}^{A_{1}} M c a p_{1 \alpha_{1} t} \leq k_{t}^{-}+\sum_{\alpha_{1}=1}^{A_{1}} \operatorname{Mcap}_{1 \alpha_{1} t} \quad \forall t \\
& \psi_{1 t} \leq k_{t}^{+} \quad \forall t \\
& \psi_{1 t} \leq \phi_{1 t} \text { Mdem } \quad \forall t \\
& \phi_{1 t} \text { Mdem }+k_{t}^{+} \leq \psi_{1 t}+\text { Mdem } \quad \forall t \\
& \psi_{2 t} \leq k_{t}^{+} \\
& \psi_{2 t} \leq \phi_{2 t} \text { Mdem } \quad \forall t \\
& \phi_{2 t} \text { Mdem }+k_{t}^{+} \leq \psi_{2 t}+\text { Mdem } \quad \forall t \\
& \psi_{3 t} \leq k_{t}^{-} \quad \forall t \\
& \psi_{3 t} \leq \phi_{1 t} \sum_{\alpha_{1}=1}^{A_{1}} \operatorname{Mcap}_{1 \alpha_{1} t} \quad \forall t \\
& \phi_{1 t} \sum_{\alpha_{1}=1}^{A_{1}} \operatorname{Mcap}_{1 \alpha_{1} t}+k_{t}^{-} \leq \psi_{3 t}+\sum_{\alpha_{1}=1}^{A_{1}} \operatorname{Mcap}_{1 \alpha_{1} t} \quad \forall t \\
& \psi_{4 t} \leq k_{t}^{-} \quad \forall t \\
& \psi_{4 t} \leq \phi_{2 t} \sum_{\alpha_{1}=1}^{A_{1}} \operatorname{Mcap}_{1 \alpha_{1} t} \quad \forall t \\
& \phi_{2 t} \sum_{\alpha_{1}=1}^{A_{1}} \operatorname{Mcap}_{1 \alpha_{1} t}+k_{t}^{-} \leq \psi_{4 t}+\sum_{\alpha_{1}=1}^{A_{1}} \operatorname{Mcap}_{1 \alpha_{1} t} \quad \forall t \\
& x_{i j \alpha_{i} t}^{1}, u_{j k l \alpha_{k} t}, L T_{i t}, \text { ptime }_{i t}, \lambda_{1 t}, \lambda_{2 t}, \psi_{1 t}, \psi_{2 t}, \psi_{3 t}, \psi_{4 t}, k_{t}^{+}, k_{t}^{-}, \text {Demand }_{k t}, v_{t}, \delta_{n t} \geq 0 \text {; } \\
& \pi_{i \alpha_{i} t}, y_{j l}, \tau_{t}^{+}, \tau_{t}^{-}, \phi_{1 t}, \phi_{2 t}, \gamma_{n t} \in\{0,1\}
\end{aligned}
$$




\section{B.2 REDUCED DFSC MODEL - 2}

The following mathematical model is the reduced DFSC model that reflects the removal of lead time and inventory concepts from the complete model. 
$\max \sum_{t=1}^{T} \omega_{1 t}\left(k_{t}^{+}+k_{t}^{-}\right)+\left(\omega_{2 t}-\omega_{1 t}\right)\left(\psi_{1 t}+\psi_{3 t}\right)+\left(\omega_{3 t}-\omega_{1 t}\right)\left(\psi_{2 t}+\psi_{4 t}\right)-$

$\left(\sum_{t=1}^{T} \sum_{i=1}^{P} \sum_{j=1}^{S} \sum_{\alpha_{i}=1}^{A_{i}}\left(c_{i j \alpha_{i} t}^{1} x_{i j \alpha_{i} t}^{1}+c_{i j \alpha_{i} t}^{2} x_{i j \alpha_{i} t}^{2}\right)+\sum_{j=1}^{S} \sum_{l=1}^{S} y_{j l}\right.$ Netwco $_{j l}+\sum_{t=1}^{T} \sum_{j=1}^{S} \sum_{k=1}^{P} \sum_{l=1}^{S} \sum_{\alpha_{k}}^{A_{k}} u_{j k l \alpha_{k} t}$ Tportcost $\left._{j k l \alpha_{k} t}\right)$

s.t.

Demand $_{1 t}=\left(\left(\beta_{1} \omega_{1 t}^{2}+\beta_{2}\right) v_{t}+\beta_{1} \lambda_{1 t}\left(\omega_{2 t}^{2}-\omega_{1 t}^{2}\right)+\beta_{1} \lambda_{2 t}\left(\omega_{3 t}^{2}-\omega_{1 t}^{2}\right)\right) \times$ timemultip lier $_{t} \quad \forall t$ (A.72)

Demand $_{k t}=\sum_{\mathrm{i}=1}^{\mathrm{k}-1}$ Demand $_{i t}$ Relation $_{i k} \quad \forall k(k>1), t$

$\sum_{\alpha_{k}=1}^{A_{k}} \sum_{j=1}^{S}\left(x_{k j \alpha_{k} t}^{1}+x_{k j \alpha_{k} t}^{2}\right) \geq \sum_{i=1}^{k-1} \sum_{\alpha_{i}=1}^{A_{i}} \sum_{j=1}^{S}\left(x_{i j \alpha_{i} t}^{1}+x_{i j \alpha_{i} t}^{2}\right)$ Relation $_{i k} \quad \forall k(k>1), t$

$\sum_{j=1}^{S} x_{i j \alpha_{i} t}^{1} \leq \pi_{i \alpha_{i} t} \operatorname{Mcap}_{i \alpha_{i} t}$

$\forall i, \alpha_{i}, t$

$\sum_{\alpha_{i}=1}^{A_{i}} \pi_{i \alpha_{i} t}=1$

$\forall i, t$

$v_{t}=\sum_{i=1}^{P} \sum_{\alpha_{i}=1}^{A_{i}} \pi_{i \alpha_{i} t} v a l_{i \alpha_{i} t}$

$\forall t$

$x_{i j \alpha_{i} t}^{1} \leq$ Capacity $_{i j \alpha_{i} t}^{1}$

$\forall i, j, \alpha_{i}, t$

$x_{i j \alpha_{i} t}^{1} \geq$ Capacity $_{i j \alpha_{i} t}^{1} a_{i j \alpha_{i} t}$

$\forall i, j, \alpha_{i}, t$

$x_{i j \alpha_{i} t}^{2} \leq$ Capacity $_{i j \alpha_{i} t}^{2} a_{i j \alpha_{i} t}$

$\forall i, j, \alpha_{i}, t$

$y_{j l}\left(\right.$ Capacity $_{k l \alpha_{k} t}^{1}+$ Capacity $\left._{k l \alpha_{k} t}^{2}\right) \geq u_{j k l \alpha_{k} t}$

$\forall j, k, l, \alpha_{k}, t$

$\sum_{j=1}^{S} u_{j k l \alpha_{k} t} \leq x_{k l \alpha_{k} t}^{1}+x_{k l \alpha_{k} t}^{2}$

$\forall k, l, \alpha_{k}, t$

$\sum_{\alpha_{k}=1}^{A_{k}} \sum_{l=1}^{S} u_{j k l \alpha_{k} t} \geq$ Relation $_{i k} \sum_{\alpha_{i}=1}^{A_{i}}\left(x_{i j \alpha_{i} t}^{1}+x_{i j \alpha_{i} t}^{2}\right) \quad \forall i, j, k, t \mid$ Relation $_{i k}>0$ 


$$
\begin{aligned}
& \phi_{1 t}+\phi_{2 t} \leq 1 \\
& \forall t \\
& \lambda_{q t} \leq \phi_{q t} \\
& \forall t, q \in\{1,2\} \\
& \lambda_{q t} \leq v_{t} \\
& \forall t, q \in\{1,2\} \\
& \phi_{q t}+v_{t} \leq \lambda_{q t}+1 \\
& \forall t, q \in\{1,2\} \\
& \tau_{t}^{+} \sum_{\alpha_{1}=1}^{A_{1}} \operatorname{Mcap}_{1 \alpha_{1} t} \geq \sum_{\alpha_{1}=1}^{A_{1}} \sum_{j=1}^{S}\left(x_{1 j \alpha_{1} t}^{1}+x_{1 j \alpha_{1} t}^{2}\right)-\text { Demand }_{1 t} \quad \forall t \\
& \tau_{t}^{-} \text {Mdem } \geq \text { Demand }_{1 t}-\sum_{\alpha_{1}=1}^{A_{1}} \sum_{j=1}^{S}\left(x_{1 j \alpha_{1} t}^{1}+x_{1 j \alpha_{1} t}^{2}\right) \quad \forall t \\
& \tau_{t}^{+}+\tau_{t}^{-} \leq 1 \\
& k_{t}^{+} \leq \text {Demand }_{1 t} \\
& k_{t}^{+} \leq \tau_{t}^{+} \text {Mdem } \\
& \text { Demand }_{1 t}+\tau_{t}^{+} \text {Mdem } \leq k_{t}^{+}+\text {Mdem } \\
& k_{t}^{-} \leq \sum_{\alpha_{1}=1}^{A_{1}} \sum_{j=1}^{S}\left(x_{1 j \alpha_{1} t}^{1}+x_{1 j \alpha_{1} t}^{2}\right) \\
& k_{t}^{-} \leq \tau_{t}^{-} \sum_{\alpha_{1}=1}^{A_{1}} \operatorname{Mcap}_{1 \alpha_{1} t} \\
& \sum_{\alpha_{1}=1}^{A_{1}} \sum_{j=1}^{S}\left(x_{1 j \alpha_{1} t}^{1}+x_{1 j \alpha_{1} t}^{2}\right)+\tau_{t}^{-} \sum_{\alpha_{1}=1}^{A_{1}} M \operatorname{cap}_{1 \alpha_{1} t} \leq k_{t}^{-}+\sum_{\alpha_{1}=1}^{A_{1}} \operatorname{Mcap}_{1 \alpha_{1} t} \quad \forall t \\
& \psi_{1 t} \leq k_{t}^{+} \\
& \forall t \\
& \psi_{1 t} \leq \phi_{1 t} \text { Mdem } \\
& \forall t \\
& \phi_{1 t} M d e m+k_{t}^{+} \leq \psi_{1 t}+\text { Mdem } \\
& \forall t \\
& \psi_{2 t} \leq k_{t}^{+} \\
& \psi_{2 t} \leq \phi_{2 t} \text { Mdem } \\
& \forall t \\
& \forall t \\
& \phi_{2 t} \mathrm{Mdem}+k_{t}^{+} \leq \psi_{2 t}+\text { Mdem } \quad \forall t \\
& \psi_{3 t} \leq k_{t}^{-} \\
& \psi_{3 t} \leq \phi_{1 t} \sum_{\alpha_{1}=1}^{A_{1}} \operatorname{Mcap}_{1 \alpha_{1} t} \\
& \phi_{1 t} \sum_{\alpha_{1}=1}^{A_{1}} \operatorname{Mcap}_{1 \alpha_{1} t}+k_{t}^{-} \leq \psi_{3 t}+\sum_{\alpha_{1}=1}^{A_{1}} \operatorname{Mcap}_{1 \alpha_{1} t} \\
& \psi_{4 t} \leq k_{t}^{-} \\
& \psi_{4 t} \leq \phi_{2 t} \sum_{\alpha_{1}=1}^{A_{1}} \operatorname{Mcap}_{1 \alpha_{1} t} \\
& \phi_{2 t} \sum_{\alpha_{1}=1}^{A_{1}} \operatorname{Mcap}_{1 \alpha_{1} t}+k_{t}^{-} \leq \psi_{4 t}+\sum_{\alpha_{1}=1}^{A_{1}} \operatorname{Mcap}_{1 \alpha_{1} t} \\
& x_{i j \alpha_{i} t}^{1}, x_{i j \alpha_{i} t}^{2}, u_{j k l \alpha_{k} t}, \lambda_{1 t}, \lambda_{2 t}, \psi_{1 t}, \psi_{2 t}, \psi_{3 t}, \psi_{4 t}, k_{t}^{+}, k_{t}^{-}, \text {Demand }_{k t}, v_{t} \geq 0 \text {; } \\
& \pi_{i \alpha_{i} t}, y_{j l}, a_{i j \alpha_{i} t}, \tau_{t}^{+}, \tau_{t}^{-}, \phi_{1 t}, \phi_{2 t} \in\{0,1\}
\end{aligned}
$$




\section{APPENDIX C}

\section{PRELIMINARY GA PARAMETER TEST RESULTS}

\begin{tabular}{rccccc}
\hline $\begin{array}{c}\text { Population } \\
\text { Size }\end{array}$ & $\begin{array}{c}\text { Number of } \\
\text { Generations }\end{array}$ & $\begin{array}{c}\text { \% Elitist } \\
\text { Chromosomes }\end{array}$ & $\begin{array}{c}\text { \% Immigrating } \\
\text { Chromosomes }\end{array}$ & $\begin{array}{c}\text { Best } \\
\text { Objective }(\$)\end{array}$ & $\begin{array}{c}\text { Solution Time } \\
\text { (in seconds) }\end{array}$ \\
\hline 100 & 1,000 & 1 & 1 & $55,008,046$ & $6,440.83$ \\
100 & 1,000 & 5 & 1 & $54,389,653$ & $3,389.32$ \\
100 & 1,000 & 10 & 1 & $54,832,677$ & $3,435.80$ \\
100 & 1,000 & 5 & 5 & $57,267,504$ & $3,507.90$ \\
100 & 1,000 & 10 & 10 & $58,886,818$ & $3,619.83$ \\
10 & 50 & 5 & 1 & $80,069,169$ & 17.51 \\
25 & 50 & 5 & 1 & $79,495,423$ & 41.54 \\
50 & 50 & 5 & 1 & $71,512,200$ & 81.49 \\
100 & 50 & 5 & 1 & $72,920,801$ & 169.71 \\
200 & 50 & 5 & 1 & $70,655,715$ & 334.39 \\
10 & 100 & 5 & 1 & $80,839,748$ & 39.75 \\
25 & 100 & 5 & 1 & $73,995,774$ & 83.60 \\
50 & 100 & 5 & 1 & $71,460,939$ & 164.07 \\
100 & 100 & 5 & 1 & $71,755,095$ & 338.87 \\
200 & 100 & 5 & 1 & $70,504,045$ & 646.53 \\
50 & 250 & 5 & 1 & $73,099,370$ & 420.25 \\
100 & 250 & 5 & 1 & $63,283,761$ & 836.70 \\
200 & 250 & 5 & 1 & $61,447,527$ & $1,678.82$ \\
200 & 500 & 5 & 1 & $56,948,753$ & $3,153.78$ \\
50 & 1,000 & 5 & 1 & $60,943,471$ & $1,876.25$ \\
100 & 1,000 & 5 & 1 & $54,389,653$ & $3,389.00$ \\
50 & 5,000 & 5 & 1 & $55,662,800$ & $9,924.39$ \\
100 & 5,000 & 5 & 1 & $49,263,967$ & $18,966.84$ \\
200 & 5,000 & 5 & $49,553,641$ & $38,692.77$ \\
\hline
\end{tabular}

* Shaded areas represent tested parameter. (The rest of the parameters are kept constant.)

** Starting mutation rate is set at $5 \%$. 


\section{APPENDIX D}

\section{REDUCED GA SUB-MODELS}

This appendix presents the reduced MIP models that are used in the GA procedure. Both models have fewer variables and constraints than the complete DFSC model and the corresponding GA sub-models since linearization, economies of scale, and lead time (with inventory cost calculation) variables are eliminated simultaneously.

\section{D.1 REDUCED GA SUB-MODEL - 1}

This model removes economies of scale concept from the presented GA sub-model. 


$$
\begin{aligned}
& \max \left(\sum_{t=1}^{T} \text { price }_{t}\left(k_{t}^{+}+k_{t}^{-}\right)\right)-\left(\sum_{t=1}^{T} \sum_{i=1}^{P} \sum_{j=1}^{S} \sum_{\alpha_{i}=1}^{A_{i}} c_{i j \alpha_{i}}^{1} x_{i j \alpha_{i} t}^{1}+\sum_{j=1}^{S} \sum_{l=1}^{S} y_{j l} \text { Netwco }_{j l}+\right. \\
& \left.\sum_{t=1}^{T} \sum_{j=1}^{S} \sum_{k=1}^{P} \sum_{l=1}^{S} \sum_{\alpha_{k}}^{A_{k}} u_{j k l \alpha_{k} t} \text { Tportcost }_{j k l \alpha_{k} t}+\sum_{t=1}^{T} \frac{1}{2} h_{t}\left(1+z_{\text {ssratio }} \rho_{2}\right) \text { Demand }_{1 t} \text { LTint }_{l t}\right)
\end{aligned}
$$

s.t.

$$
\begin{aligned}
& \sum_{\alpha_{k}=1}^{A_{k}} \sum_{j=1}^{S} x_{k j \alpha_{k} t}^{1} \geq \sum_{i=1}^{k-1} \sum_{\alpha_{i}=1}^{A_{i}} \sum_{j=1}^{S} x_{i j \alpha_{i} t}^{1} \text { Relation }_{i k} \quad \forall k(k>1), t \\
& \sum_{j=1}^{S} x_{i j \alpha_{i} t}^{1} \leq \pi_{i \alpha_{i} t} \operatorname{Mcap}_{i \alpha_{i} t} \\
& \forall i, \alpha_{i}, t \\
& x_{i j \alpha_{i} t}^{1} \leq \text { Capacity }_{i j \alpha_{i} t}^{1} \quad \forall i, j, \alpha_{i}, t \\
& y_{j l} \text { Capacity }_{k l \alpha_{k} t}^{1} \geq u_{j k l \alpha_{k} t} \quad \forall j, k, l, \alpha_{k}, t \\
& \sum_{j=1}^{S} u_{j k l \alpha_{k} t} \leq x_{k l \alpha_{k} t}^{1} \quad \forall k, l, \alpha_{k}, t \\
& \sum_{\alpha_{k}=1}^{A_{k}} \sum_{l=1}^{S} u_{j k l \alpha_{k} t} \geq \text { Relation }_{i k} \sum_{\alpha_{i}=1}^{A_{i}} x_{i j \alpha_{i} t}^{1} \quad \forall i, j, k, t \mid \text { Relation }_{i k}>0 \\
& \text { ptime }_{i t} \geq \frac{p t_{i j \alpha_{i} t} x_{i j \alpha_{i} t}^{1}}{\text { Capacity }_{i j \alpha_{i} t}^{1}} \quad \forall i, j, \alpha_{i}, t \mid \text { Capacity }_{i j \alpha t}^{1}>0 \\
& L T_{i t} \geq L T_{k t} \text { Relation }_{i k}+\text { ptime }_{i t} \quad \forall i, k, t \mid \text { Relation }_{i k}>0 \\
& L T_{i t} \geq \text { ptime }_{i t} \\
& \text { LTint }_{t} \geq L T_{1 t} \quad \forall t \\
& \tau_{t}^{+} \sum_{\alpha_{1}=1}^{A_{1}} \text { Mcap }_{1 \alpha_{1} t} \geq \sum_{\alpha_{1}=1}^{A_{1}} \sum_{j=1}^{S} x_{1 j \alpha_{1} t}^{1}-\text { Demand }_{1 t} \quad \forall t \\
& \tau_{t}^{-} \text {Mdem } \geq \text { Demand }_{1 t}-\sum_{\alpha_{1}=1}^{A_{1}} \sum_{j=1}^{S} x_{1 j \alpha_{1} t}^{1} \quad \forall t \\
& \tau_{t}^{+}+\tau_{t}^{-} \leq 1 \quad \forall t \\
& k_{t}^{+} \leq \text {Demand }_{1 t} \quad \forall t \\
& k_{t}^{+} \leq \tau_{t}^{+} \text {Mdem } \quad \forall t \\
& \text { Demand }_{1 t}+\tau_{t}^{+} \text {Mdem } \leq k_{t}^{+}+\text {Mdem } \quad \forall t \\
& k_{t}^{-} \leq \sum_{\alpha_{1}=1}^{A_{1}} \sum_{j=1}^{S} x_{1 j \alpha_{1} t}^{1} \quad \forall t \\
& k_{t}^{-} \leq \tau_{t}^{-} \sum_{\alpha_{1}=1}^{A_{1}} \operatorname{Mcap}_{1 \alpha_{1} t} \quad \forall t \\
& \sum_{\alpha_{1}=1}^{A_{1}} \sum_{j=1}^{S} x_{1 j \alpha_{1} t}^{1}+\tau_{t}^{-} \sum_{\alpha_{1}=1}^{A_{1}} \operatorname{Mcap}_{1 \alpha_{1} t} \leq k_{t}^{-}+\sum_{\alpha_{1}=1}^{A_{1}} \operatorname{Mcap}_{1 \alpha_{1} t} \quad \forall t \\
& \forall i, t \mid \text { Relation }_{i k}=0 \text { for } \forall k>i \\
& \forall t \\
& \forall t \quad \text { (A.121) } \\
& \forall t \quad \text { (A.122) } \\
& \forall t \\
& \text { (A.124) } \\
& \forall t \quad \text { (A.125) } \\
& \forall t \quad \text { (A.126) } \\
& \text { (A.127) } \\
& x_{i j \alpha_{i} t}^{1}, u_{j k l \alpha_{k} t}, L T_{i t}, \text { ptime }_{i t}, k_{t}^{+}, k_{t}^{-}, \text {Demand }_{k t} \geq 0 ; \text { Lint }_{t} \geq 0 \text { and integer; } \pi_{i \alpha_{i} t}, y_{j l}, \tau_{t}^{+}, \tau_{t}^{-} \in\{0,1\}
\end{aligned}
$$




\section{D.2 REDUCED GA SUB-MODEL - 2}

This model removes lead time and inventory cost concepts from the presented GA sub-model.

$$
\begin{aligned}
& \max \left(\sum_{t=1}^{T} \text { price }_{t}\left(k_{t}^{+}+k_{t}^{-}\right)\right)-\left(\sum_{t=1}^{T} \sum_{i=1}^{P} \sum_{j=1}^{S} \sum_{\alpha_{i}=1}^{A_{i}}\left(c_{i j \alpha_{i} t}^{1} x_{i j \alpha_{i} t}^{1}+c_{i j \alpha_{i} t}^{2} x_{i j \alpha_{i} t}^{2}\right)+\sum_{j=1}^{S} \sum_{l=1}^{S} y_{j l} \text { Netwco }_{j l}+\right. \\
& \left.\sum_{t=1}^{T} \sum_{j=1}^{S} \sum_{k=1}^{P} \sum_{l=1}^{S} \sum_{\alpha_{k}}^{A_{k}} u_{j k l \alpha_{k}} T_{\text {portcost }}{ }_{j k l \alpha_{k} t}\right)
\end{aligned}
$$

s.t.

$\sum_{\alpha_{k}=1}^{A_{k}} \sum_{j=1}^{S}\left(x_{k j \alpha_{k} t}^{1}+x_{k j \alpha_{k} t}^{2}\right) \geq \sum_{i=1}^{k-1} \sum_{a_{i}=1}^{A_{i}} \sum_{j=1}^{S}\left(x_{i j \alpha_{i} t}^{1}+x_{i j \alpha_{i} t}^{2}\right)$ Relation $_{i k} \quad \forall k(k>1), t$

$\sum_{j=1}^{S} x_{i j \alpha_{i} t}^{1} \leq \pi_{i \alpha_{i} t} \operatorname{Mcap}_{i \alpha_{i} t}$

$\forall i, \alpha_{i}, t$

$x_{i j \alpha_{i}}^{1} \leq$ Capacity $y_{i j \alpha_{i} t}^{1}$

$\forall i, j, \alpha_{i}, t$

$x_{i j \alpha_{i} t}^{1} \geq$ Capacity $_{i j \alpha_{i} t}^{1} a_{i j \alpha_{i} t}$

$\forall i, j, \alpha_{i}, t$

$x_{i j \alpha_{i} t}^{2} \leq$ Capacity $_{i j \alpha_{i} t}^{2} a_{i j \alpha_{i} t}$

$\forall i, j, \alpha_{i}, t$

$y_{j l}\left(\right.$ Capacity $_{k l \alpha_{k} t}^{1}+$ Capacity $\left._{k l \alpha_{k} t}^{2}\right) \geq u_{j k l \alpha_{k} t}$

$\forall j, k, l, \alpha_{k}, t$

$\sum_{j=1}^{S} u_{j k l \alpha_{k} t} \leq x_{k l \alpha_{k} t}^{1}+x_{k l \alpha_{k} t}^{2}$

$\forall k, l, \alpha_{k}, t$

$\sum_{\alpha_{k}=1}^{A_{k}} \sum_{l=1}^{S} u_{j k l \alpha_{k} t} \geq$ Relation $_{i k} \sum_{\alpha_{i}=1}^{A_{i}}\left(x_{i j \alpha_{i} t}^{1}+x_{i j \alpha_{i} t}^{2}\right)$

$\forall i, j, k, t \mid$ Relation $_{i k}>0$

$\tau_{t}^{+} \sum_{\alpha_{1}=1}^{A_{1}} \operatorname{Mcap}_{1 \alpha_{1} t} \geq \sum_{\alpha_{1}=1}^{A_{1}} \sum_{j=1}^{S}\left(x_{1 j \alpha_{1} t}^{1}+x_{1 j \alpha_{1} t}^{2}\right)-$ Demand $_{1 t}$

$\forall t$

$\tau_{t}^{-}$Mdem $\geq$Demand $_{1 t}-\sum_{\alpha_{1}=1}^{A_{1}} \sum_{j=1}^{S}\left(x_{1 j \alpha_{1} t}^{1}+x_{1 j \alpha_{1}}^{2}\right)$

$\forall t$

$\tau_{t}^{+}+\tau_{t}^{-} \leq 1$

$\forall t$

$k_{t}^{+} \leq$Demand $_{1 t}$

$\forall t$

$k_{t}^{+} \leq \tau_{t}^{+} \mathrm{Mdem}$

$\forall t$

Demand $_{1 t}+\tau_{t}^{+}$Mdem $\leq k_{t}^{+}+$Mdem

$\forall t$

$k_{t}^{-} \leq \sum_{\alpha_{1}=1}^{A_{1}} \sum_{j=1}^{S}\left(x_{1 j \alpha_{1} t}^{1}+x_{1 j \alpha_{1} t}^{2}\right)$

$\forall t$

$k_{t}^{-} \leq \tau_{t}^{-} \sum_{\alpha_{1}=1}^{A_{1}}$ Mcap $_{1 \alpha_{1} t}$

$\sum_{\alpha_{1}=1}^{A_{1}} \sum_{j=1}^{S}\left(x_{1 j \alpha_{1} t}^{1}+x_{1 j \alpha_{1} t}^{2}\right)+\tau_{t}^{-} \sum_{\alpha_{1}=1}^{A_{1}} M c a p_{1 \alpha_{1} t} \leq k_{t}^{-}+\sum_{\alpha_{1}=1}^{A_{1}} M c a p_{1 \alpha_{1} t} \quad \forall t$

$x_{i j \alpha_{i} t}^{1}, x_{i j \alpha_{i} t}^{2}, u_{j k l \alpha_{k} t}, k_{t}^{+}, k_{t}^{-}$, Demand $_{k t} \geq 0 ; \quad \pi_{i \alpha_{i} t}, y_{j l}, a_{i j \alpha_{i} t}, \tau_{t}^{+}, \tau_{t}^{-} \in\{0,1\}$ 


\section{BIBLIOGRAPHY}

[1] Alibre Inc., "Design Anywhere. Build Anywhere. The Opportunity in Global Manufacturing," 2001, http://www.alibre.com.

[2] Arntzen, B. C., Brown, G. G., Harrison, T. P., Trafton, L. L., "Global Supply Chain Management at Digital Equipment Corporation,” Interfaces, Vol. 25, No. 1, pp. 69-93, 1995.

[3] Asiedu, Y., Gu, P., "Product Life Cycle Cost Analysis: State of the Art Review," International Journal of Production Review, Vol. 36, No. 4, pp. 883 - 908, 1998.

[4] Aytug, H., Khouja, M., Vergara, F. E., "Use of Genetic Algorithms to solve Production and Operations Management Problems: A Review," International Journal of Production Research, Vol. 41, No. 17, pp. 3955-4009, 2003.

[5] Babcock, D., L., Morse, L. C., Managing Engineering and Technology, $3^{\text {rd }}$ Edition, Prentice Hall, Upper Saddle River, NJ, 2002.

[6] Baz, M., Brooks, J. P., Gosavi, A., Hunsaker, B., “Automated Tuning of Optimization Software Parameters," Technical Report 2007-7, University of Pittsburgh Department of Industrial Engineering.

[7] Beamon, B. M., "Supply Chain Design and Analysis: Models and Methods," International Journal of Production Economics, Vol. 55, No. 3, pp. 281-294, 1998.

[8] Berger, A. J., Gattorna, J. L., Supply Chain Cybermastery: Building high performance supply chains of the future, Gower Publishing Limited, Burlington, VT, 2001.

[9] Besterfield, D., H., Besterfield-Michna, C., Besterfield, G., H., Besterfield-Sacre, M., Total Quality Management, $3^{\text {rd }}$ Edition, Prentice Hall, Upper Saddle River, NJ, 2003.

[10] Cakravista, A., Toha, I. S., Nakamura, N., "A Two-Stage Model for the Design of Supply Chain Networks," International Journal of Production Economics, Vol. 80, No. 3, pp. 231-248, 2002.

[11] Chan, K. Y., Aydin, M. E., Fogarty, T. C., "Main effect fine-tuning of the mutation operator and the neighborhood function for uncapacitated facility location problems," Soft Computing, Vol. 10, No. 11, pp. 1075-1090, 2006. 
[12] Chan, L., Wu, M., "Quality Function Deployment: A Literature Review," European Journal of Operational Research, Vol. 143, No. 3, pp. 463-497, 2002.

[13] Chopra, S., Meindl, P., Supply Chain Management: Strategy, Planning, and Operation, $2^{\text {nd }}$ Edition, Pearson/Prentice Hall, Upper Saddle River, NJ, 2004.

[14] Clark, A. J., Scarf, H., "Optimal Policies for a Multi-Echelon Inventory Problem," Management Science, Vol. 6, No. 4, pp. 475-490, 1960.

[15] Coello C. A., "A Comprehensive Survey of Evolutionary-Based Multiobjective Optimization Techniques," Knowledge and Information Systems: An International Journal, Vol. 1, No. 3, pp. 269-308, 1999.

[16] Cohen, M. A., Lee, H. L., "Strategic Analysis of Integrated Production Distribution System: Models and Methods," Operations Research, Vol. 36, No. 2, pp. 216-228, 1988.

[17] Cook, H., Wu, A., "On the Valuation of Goods and Selection of the best Design Alternative," Research in Engineering Design, Vol. 13, No. 1, pp. 42-54, 2001.

[18] Cooper, R., Slagmulder, R., Target Costing and Value Engineering, Productivity Press, Portland, OR, 1997.

[19] Council of Supply Chain Management Professionals, " $18^{\text {th }}$ Annual Logistics State of Logistics Report," 2006, available at http://cscmp.org.

[20] Ding, H., Benyoucef, L., Xie, X., "A Simulation Optimization Methodology for Supplier Selection Problem,", International Journal of Integrated Manufacturing, Vol. 18, No. 2-3, pp. 210-224, 2005.

[21] Ealey, L. A., Quality by Design: Taguchi Methods and U.S. Industry, ASI Press, Dearborn, MI, 1988.

[22] Fandel, G., Stammen, M., "A General Model for Extended Strategic Supply Chain Management with Emphasis on Product Life Cycles including Development and Recycling," International Journal of Production Economics, Vol. 89, No. 3, pp. 293-308, 2004.

[23] Fisher, M. L., "What is the Right Supply Chain for Your Product?," Harvard Business Review, Vol. 75, No. 2, pp. 105-116, 1997.

[24] Geoffrion, A., M., Graves, G. W., "Multicommodity Distribution System Design by Bender's Decomposition," Management Science, Vol. 20, No. 5, pp. 822-844, 1974.

[25] Glover, F., "Future Paths for Integer Programming and Links to Artificial Intelligence," Computers \& Operations Research, Vol.13, No. 5, pp. 533-549, 1986.

[26] Glover, F. Laguna, M., "Tabu Search," Chapter 3 in Modern Heuristic Techniques for Combinatorial Problems, Reeves, C., R. (ed.), Blackwell Scientific Publications, Oxford, UK, 1993. 
[27] Goldberg, D. E., Genetic Algorithms in Search, Optimization, and Machine Learning, Addison - Wesley, Reading, Mass, 1989.

[28] Gomory, R. E., "An Algorithm for Integer Solutions to Linear Programs," Recent Advances in Mathematical Programming, R. L. Graves and P. Wolde (ed.), McGraw-Hill, New York, NY, 1963.

[29] Govers, C. P. M., "What and How About Quality Function Deployment (QFD)," International Journal of Production Economics, Vol. 46-47, No. 1, pp. 575-585, 1996.

[30] Graves, S. C., Willems, S. P., "Optimizing the Supply Chain Configuration for New Products," Management Science, Vol. 51, No. 8, pp. 1165-1180, 2005.

[31] Hanssmann, F., "Optimal Inventory Location and Control in Production and Distribution Networks," Operations Research, Vol. 7, No. 4, pp. 483-498, 1959.

[32] Hausman, W., Carison, R., Kouvelis, P., Supply Chain Seminars, 2001, http://www.supplychainseminars.com.

[33] Holland, J. H., Adaptation in Natural and Artificial Systems, University of Michigan Press, Ann Arbor., 1975.

[34] Keys, L. K., "System Life Cycle Engineering and DF'X'," IEEE Transactions on Components, Hybrids, and Manufacturing Technology, Vol. 13, No. 1, pp. 83-93, 1990.

[35] Kim, B., Leung, J. M. Y., Park, K. T., Zhang, G., Lee, S., "Configuring a Manufacturing Firm's Supply Network with Multiple Suppliers," IIE Transactions, Vol.34, No. 8, pp. 663677, 2002.

[36] Lambert, D., M., Cooper, M., C., "Issues in Supply Chain Management," Industrial Marketing Management, Vol. 29, No. 1, pp. 65-83, 2000.

[37] Lamothe, J., Hadj-Hamou, K., Aldanondo, M., "An Optimization Model for Selecting a Product Family and Designing Its Supply Chain," European Journal of Operational Research, Vol. 169, No. 3, pp. 1030-1047, 2006.

[38] Land, A. H., Doig, A. G., "An automatic method for solving discrete programming problems," Econometrica, Vol. 28, No.3, pp. 497-520, 1960.

[39] Lee, H. L., "Effective Inventory and Service Management through Product and Process Redesign," Operations Research, Vol. 44, No. 1, pp. 151-159, 1996.

[40] Lee, H. L., "Design for Supply Chain Management: Concepts and Examples," Perspectives in Operations Management: Essays in Honor of Elwood S. Buffa, R. Sarin (ed.), Kluwer Academic, Boston, MA, pp. 45-65, 1993.

[41] Lee, H. L., Billington, C., "Managing Supply Chain Inventory: Pitfalls and Opportunities," Sloan Management Review, Vol. 33, No. 3, pp. 65-73, 1992. 
[42] Lee, H. L., Billington, C., "The Evolution of Supply-Chain Management Models and Practice at Hewlett-Packard," Interfaces, Vol. 25, No. 5, pp. 42-63, 1995.

[43] Lee, H. L., Billington, C., Carter, B., "Hewlett-Packard gains control of inventory and service through design for localization," Interfaces, Vol. 23, No. 4, pp. 1-11, 1993.

[44] Lee, H. L., Sasser, M. M., "Product Universality and Design for Supply Chain Management," Production Planning \& Control, Vol. 6, No. 3, pp. 270-277, 1995.

[45] Lee, Y. H., Kim, S. H., "Production - Distribution Planning in Supply Chain Considering Capacity Constraints," Computers \& Industrial Engineering, Vol. 43, No. 1, pp 169-190, 2002.

[46] Lourenco, H. R., "Supply Chain Management: An Opportunity for Metaheuristics," UPF Economics and Business, Working Paper No. 538, 2001.

[47] Martin, M. V., Ishii, K., "Design for Variety: Development of Complexity Indices and Design Charts," Proceedings of 1997 ASME Design Engineering Technical Conferences, Paper No. DETC97/DFM-4359, Sacramento, CA, September 1997.

[48] Mentzer, J. T., Supply Chain Management, Sage Publications Inc., Thousand Oaks, CA, 2001.

[49] Perreault, W. D., McCarthy, J. E., Essentials of Marketing: A Global-Managerial Approach, $8^{\text {th }}$ Edition, Irwin/McGraw-Hill, Boston, MA, 2000.

[50] Revees, C. R., "A Genetic Algorithm for flowshop sequencing," Computers and Operations Research, Vol. 22, No. 1, pp. 5-13, 1995.

[51] Simchi-Levi, D., Kaminsky, P., Simchi-Levi, E., Designing and Managing the Supply Chain, McGraw-Hill, New York, NY, 2000.

[52] Stanford Global Supply Chain Forum, http://www.stanford.edu/group/scforum/, 2001

[53] Supply-Chain Council, http://www.supply-chain.org, 2005.

[54] Talluri S., Baker, R. C., "A Multi-Phase Mathematical Programming Approach for Effective Supply Chain Design," European Journal of Operational Research, Vol. 141, No. 3, pp. 544-558, 2002.

[55] Thomas, D. J., Griffin, P. M., “Coordinated Supply Chain Management,” European Journal of Operational Research, Vol. 94, No. 1, pp. 1-15, 1996.

[56] Truong, T. H., Azadivar, F., "Optimal Design Methodologies for Configuration of Supply Chains," International Journal of Production Research, Vol. 43, No. 11, pp. 2217-2236, 2005. 
[57] Vergara, F. E., Khouja, M, Michalewicz, Z., "An Evolutionary Algorithm for Optimizing Material Flow in Supply Chains," Computers \& Industrial Engineering, Vol. 43, No. 3, pp. 407-421, 2002.

[58] Vidal, C. J, Goetschalckx, M., "A Global Supply Chain Model with Transfer Pricing and Transportation Cost Allocation," European Journal of Operational Research, Vol. 129, No.1, pp. 134-158, 2001.

[59] Vidal, C. J, Goetschalckx, M., "Strategic Production-Distribution Models: A Critical Review with Emphasis on Global Supply Chain Models," European Journal of Operational Research, Vol. 98, No. 1, pp. 1-18, 1997.

[60] Viswanadham, N., Raghavan, NR. S., "Performance Analysis and Design of Supply Chains: A Petri Net Approach," Journal of the Operational Research Society, Vol. 51, No. 10, pp. 1158-1169, 2000.

[61] Wang, G., Huang, S. H., Dismukes, J. P., "Product-driven Supply Chain Selection Using Integrated Multi-Criteria Decision Making Methodology," International Journal of Production Economics, Vol. 91, No.1, pp. 1-15, 2004.

[62] Weber, C., Current, J. R., Benton, W. C., "Vendor Selection Criteria and Methods," European Journal of Operational Research, Vol. 50, No. 1, pp. 2-18, 1991.

[63] Winston, W. L., Operations Research: Applications and Algorithms, $3^{\text {rd }}$ ed., Duxbury Press, Belmont, CA, 1994.

[64] Wu, T., O'Grady, P., “A Methodology for improving the Design of a Supply Chain,” International Journal of Computer Integrated Manufacturing, Vol. 17, No. 4, pp. 281-293, 2004.

[65] Zeng, Y., Gu, P., "A Science-Based approach to Product Design Theory Part I: Formulation and Formalization of Design Process," Robotics and Computer Integrated Manufacturing, Vol. 15, No. 4, pp. 331-339, 1999. 